\title{
A PHENOMENOLOGICAL CASE STUDY OF A GRADUATE COMPREHENSIVE DEAF EDUCATION TEACHER PREPARATION PROGRAM: IMPLICATIONS FOR COLLABORATIVE EDUCATIONAL LEADERSHIP IN DIVERSE CONTEXTS
}

A Dissertation
presented to
the Faculty of the Graduate School
at the University of Missouri-Columbia
In Partial Fulfillment
of the Requirements for the Degree
Doctor of Education
KAREN S. ENGLER
Dr. Cynthia MacGregor, Dissertation Supervisor
July


(C) Copyright by Karen S. Engler 2014

All Rights Reserved 
The undersigned, appointed by the dean of the Graduate School, have examined the dissertation entitled

\section{A PHENOMENOLOGICAL CASE STUDY OF A GRADUATE COMPREHENSIVE DEAF EDUCATION TEACHER PREPARATION PROGRAM: IMPLICATIONS FOR COLLABORATIVE EDUCATIONAL LEADERSHIP IN DIVERSE CONTEXTS}

presented by Karen S. Engler

a candidate for the degree of doctor of education, and hereby certify that, in their opinion, it is worthy of acceptance.

Professor Cynthia MacGregor

Professor Robert Watson

Professor Beth Hurst

Professor Brian Sims 


\title{
DEDICATION
}

\author{
In honor of God \\ I dedicate my dissertation to
}

My family:

My Husband

To John, my high school sweetheart, thank you for your unwavering love and belief in me...always!

I love you!

Our Children

To Zachary, Jenna, Nicholas, and Michael

thank you for the endless joy you have added to my life...

Forever love!

To Jillian and John Ryan, our babies who took a piece of my heart with them to heaven...

Forever love!

To Ryan, who has always been like a son to us...

Forever love!

And:

To the babies, children, and students who are d/Deaf or hard of hearing \& their families, thank you for allowing me to serve you. It has been a privilege and an honor! 


\section{ACKNOWLEDGEMENTS}

There are several professors whom I would like to acknowledge for their significant contributions throughout my doctoral journey. I will begin with those who provided encouragement, guidance, and their expertise throughout coursework and the culminating pillar of this process, the dissertation. As my dissertation chair, Dr. Cynthia MacGregor, had an amazing gift for navigating with steadfast precision in a way that honored my path and vision. Her ability to trust, in the process and in me, was pivotal throughout my dissertation process and will not be forgotten. Dr. Robert Watson, fondly known as "Dr. Bob" had a passion for people which brought Cohort 8 together. He was skilled at seeing the big picture and sharing insights to make a difference. It was an absolute privilege to learn from both Dr. MacGregor and Dr. Bob.

Aside from Dr. MacGregor's leadership as my dissertation chair, I acknowledge the contributions of the remaining dissertation team members: Dr. Bob, Dr. Beth Hurst, and Dr. Brian Sims. Each provided their time and expertise resulting in a significantly improved dissertation outcome. I also recognize two more professors, Dr. Casandra Harper and Dr. Rozana Carducci, who contributed to my learning. And to Cohort 8 friends, Tracy Cleveland and Jeremy Schenk, I thank you for your support and laughter!

I did not consider pursuing a doctoral degree or successfully complete one without the support and encouragement of others at the university. Thanks to my esteemed colleagues and friends: department chair, Dr. Letitia White, deaf education professors, Dr. Ye "Angel” Wang and Tara Oetting, M.S., clinic director, Jill Oswalt, M.S., and long-time mentor, Dr. Harold Meyers. It has been an honor to have worked alongside some of the very best! Thanks to my friend, Cindy, for her ongoing support and prayers throughout my doctoral work. Also, thanks to GA's, Sonia Arora and Rachel Ball. 


\section{TABLE OF CONTENTS}

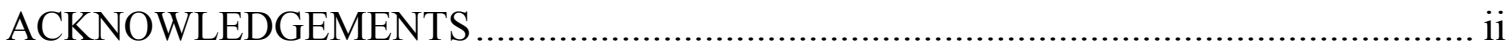

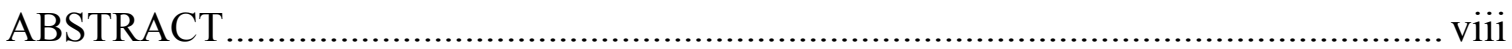

\section{Chapter}

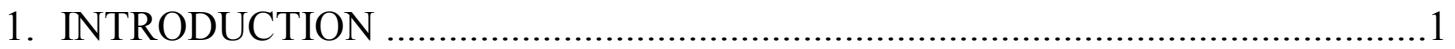

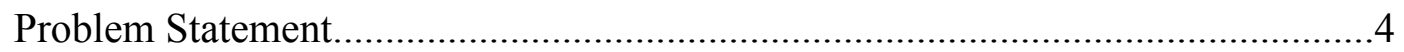

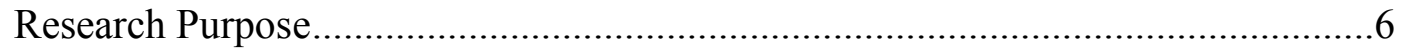

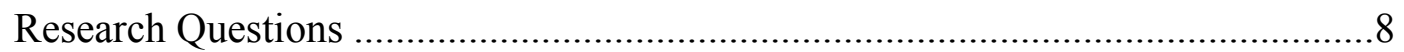

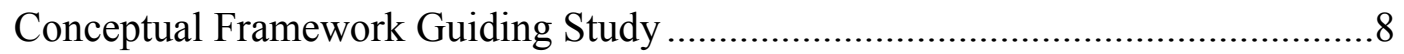

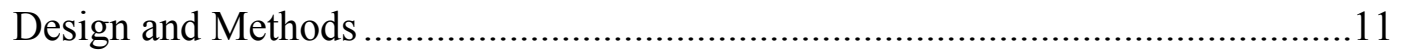

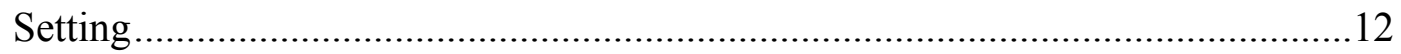

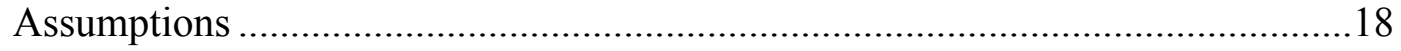

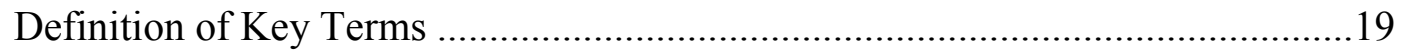

Description of Deafness ……………………………....................................19

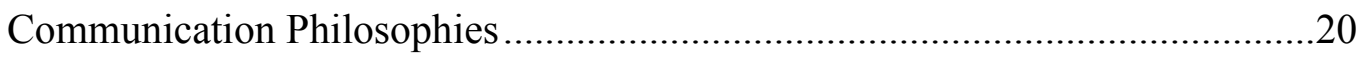

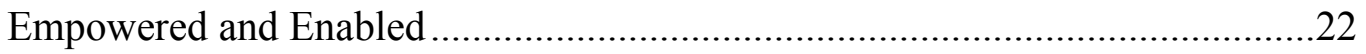

Significance of the Research for Leadership Practice ............................................24

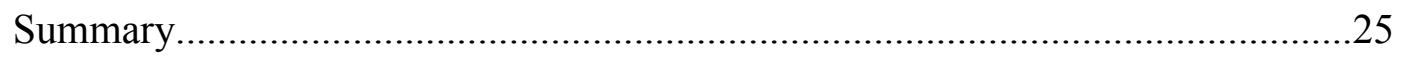

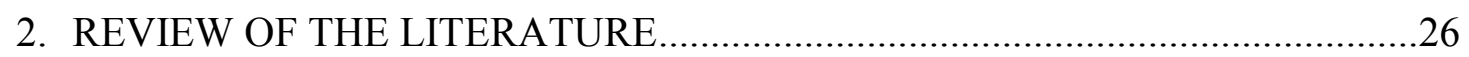

Audiometrically Defined Hearing Acuity …………………................................29

Normal Hearing Acuity: 0-15 dB HL for Children .............................................30

Slight or Minimal Hearing Loss: 15-25 dB HL for Children ................................30

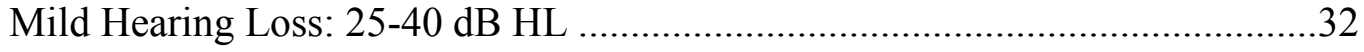

Moderate Hearing Loss: 40-55 dB HL ..........................................................3 
Moderately Severe Hearing Loss: 55-70 dB HL

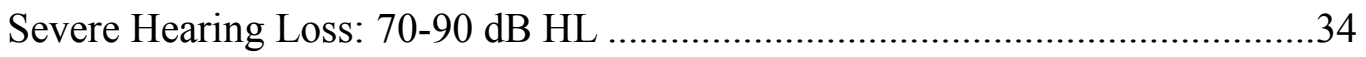

Profound Hearing Loss: 90-db HL or Greater......................................................35

Educating Students who are d/Deaf or hard of hearing ......................................36

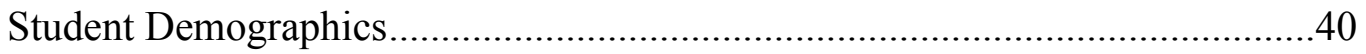

Language and Early Intervention ......................................................................44

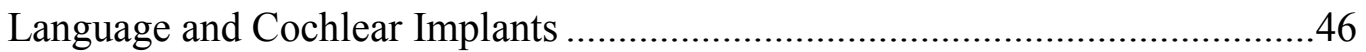

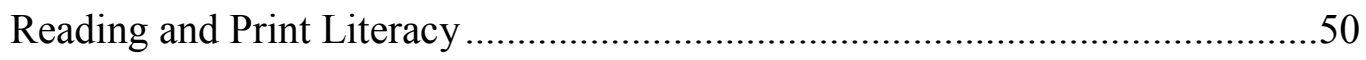

Educating Deaf Education Teacher Candidates ..................................................56

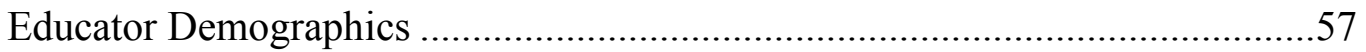

Educator's Need for Increased Knowledge, Skills, and Experiences .................60

Deaf Education Teacher Preparation Program Functioning..................................64

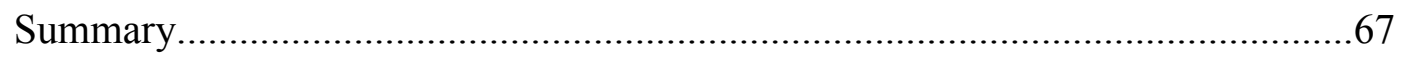

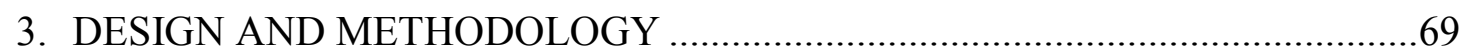

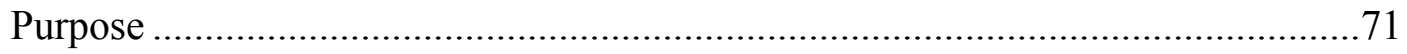

Research Questions ............................................................................................72

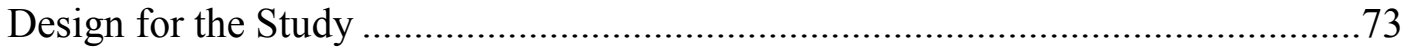

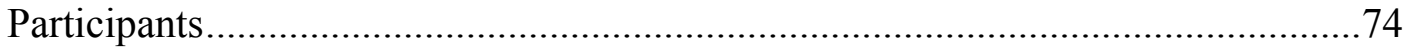

Internal Stakeholder Participants.....................................................................

External Stakeholder Participants.....................................................................

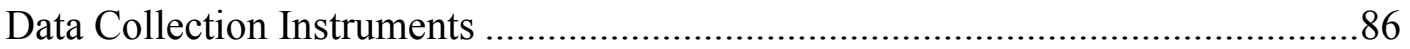

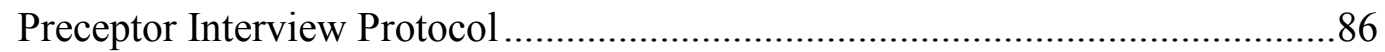

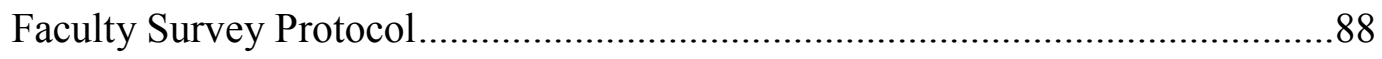

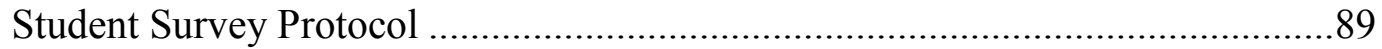




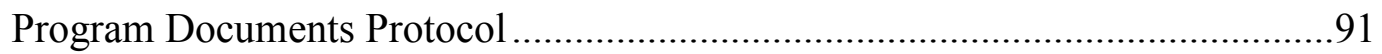

Preschool Evaluation of Services Document Protocol ........................................91

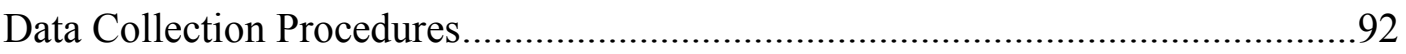

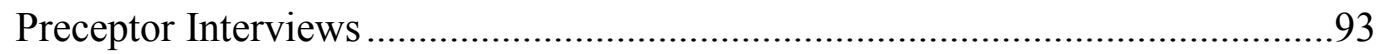

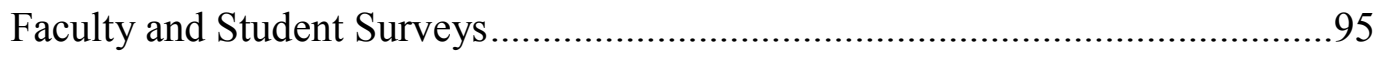

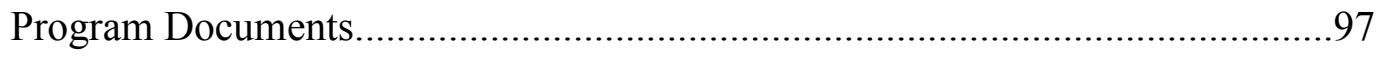

Human Subjects Protection and Other Ethical Considerations..............................98

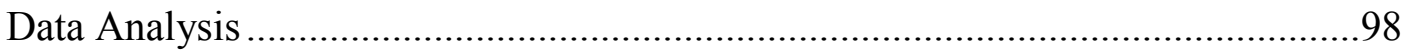

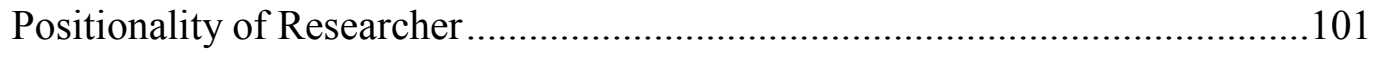

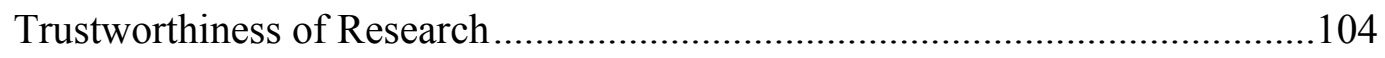

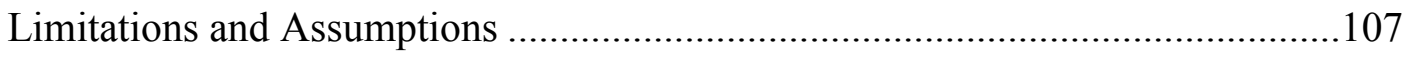

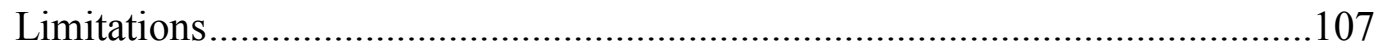

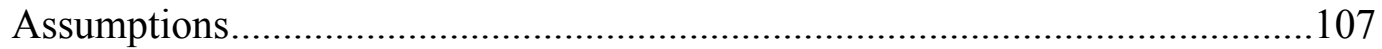

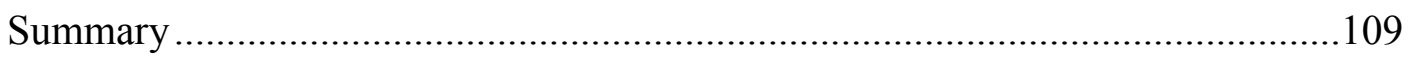

4. PRESENTATION OF FINDINGS .............................................................

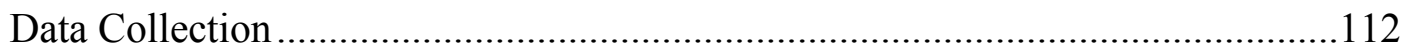

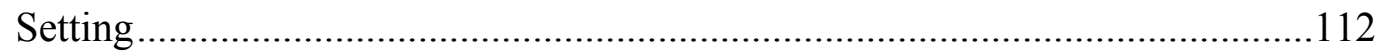

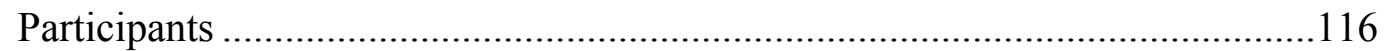

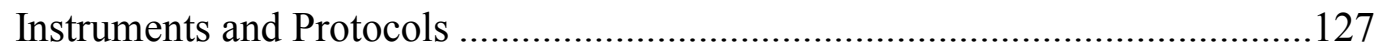

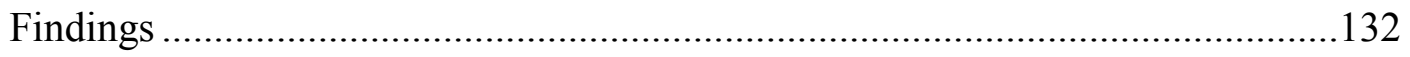

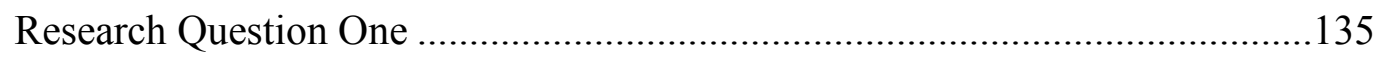

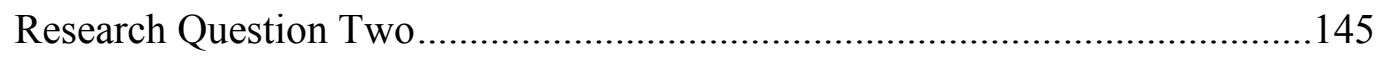

Research Question Three................................................................................159

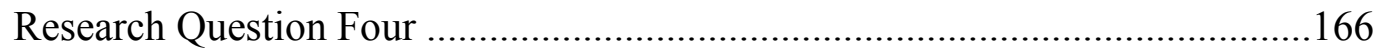




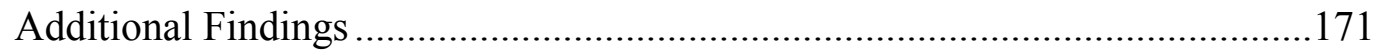

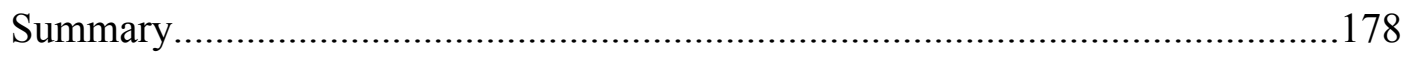

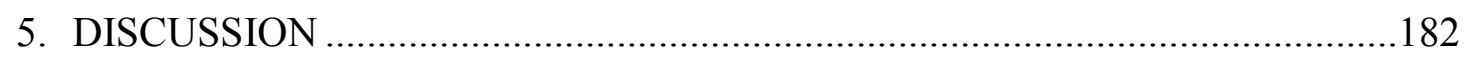

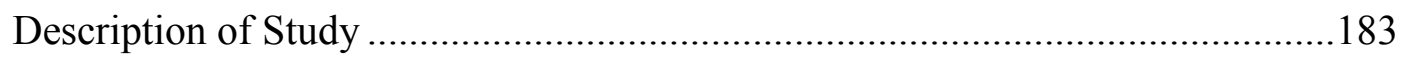

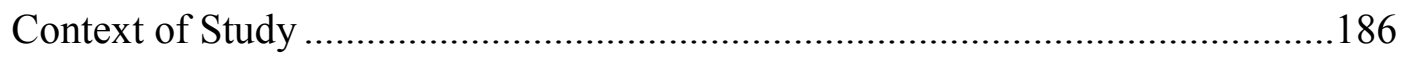

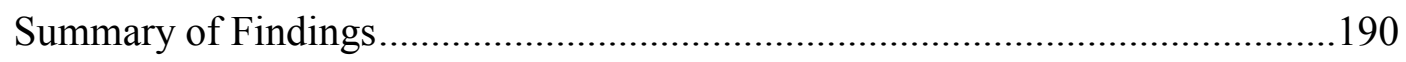

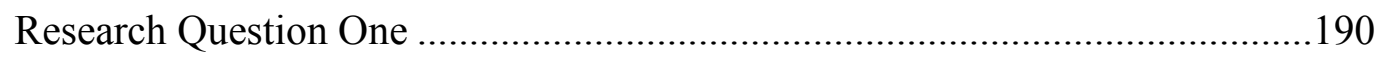

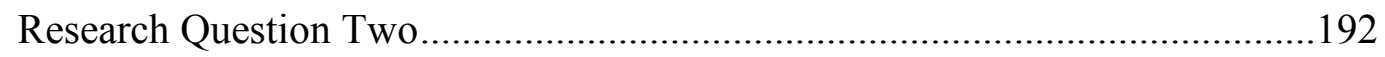

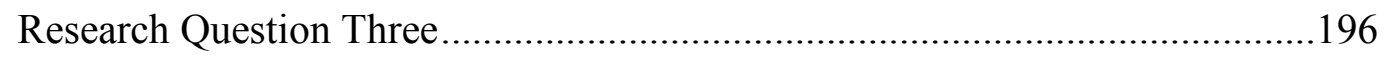

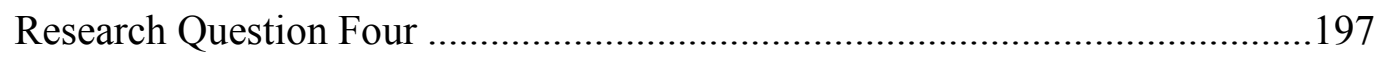

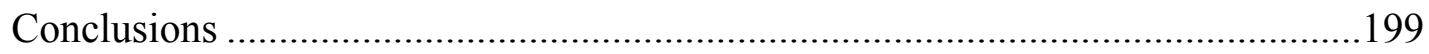

Research Question One: Commitment to the Profession .................................199

Research Question Two: Proficiency in Practice ...........................................200

Research Question Three: Learning Impact .................................................202

Research Question Four: Program Improvement .........................................202

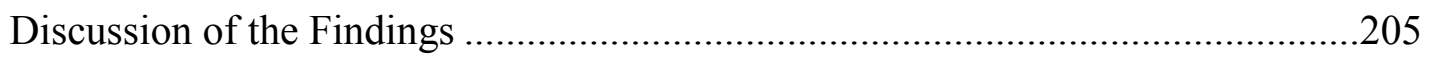

Evidence of Alignment and Non-alignment with Related Literature................205

Usefulness of the Conceptual Framework of the Study .................................210

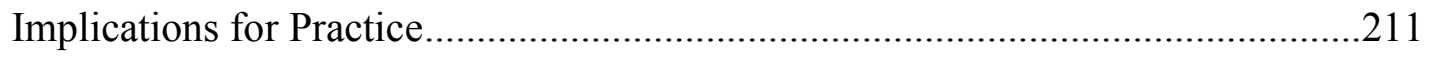

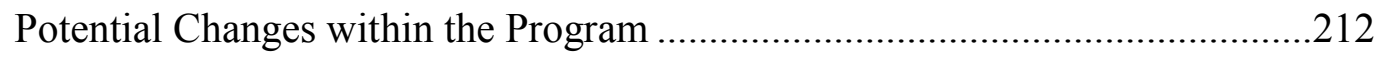

Potential Changes within the State ...........................................................213

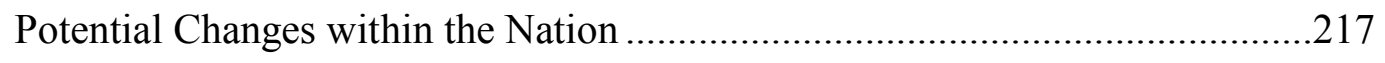

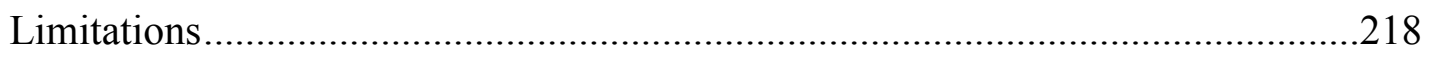

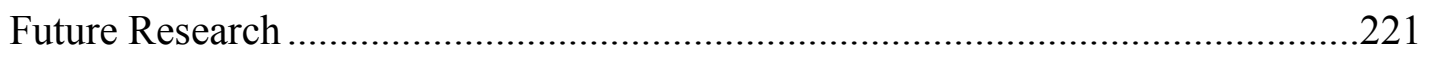

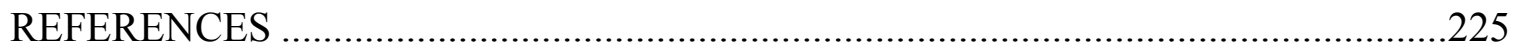


Appendix A: Preceptor Interview Consent to Participate in Research

Appendix B: Faculty Survey Consent to Participate in Research.

Appendix C: Student Survey Consent to Participate in Research

Appendix D: Preceptor Interview Protocol - Part One .246

Appendix E: Preceptor Interview Protocol - Part Two

Appendix F: Faculty Survey Protocol. .250

Appendix G: Student Survey Protocol. .253

Appendix H: Programs Documents Protocol .257

Appendix I: Preschool Evaluation of Services Document Protocol .258

Appendix J: Preceptor Interview Summary Sheet..... .259

Appendix K: Faculty Interview Summary Sheet..... .261

Appendix L: Student Interview Summary Sheet .263

Appendix M: Student Mid-term Evaluation/Final Evaluation... .265 


\title{
A PHENOMENOLOGICAL CASE STUDY OF A GRADUATE COMPREHENSIVE DEAF EDUCATION TEACHER PREPARATION PROGRAM: IMPLICATIONS FOR COLLABORATIVE EDUCATIONAL LEADERSHIP IN DIVERSE CONTEXTS
}

\author{
Karen S. Engler \\ Dr. Cynthia MacGregor, Dissertation Supervisor
}

\begin{abstract}
There exists a critical shortage of teachers of students who are $d /$ Deaf or hard of hearing in the United States; yet, deaf education teacher preparation programs are on the decline. Little is known about the functioning of deaf education teacher preparation programs that exist, namely, how they prepare teacher candidates while simultaneously providing services to learners who are d/Deaf or hard of hearing. A phenomenological case study of a Midwest, graduate, comprehensive deaf education teacher preparation program was conducted. This study explored the essence of empowered and enabled learning of program teacher candidates centered on MO-DESE's educator pillars deemed critical to the development of quality teachers. Data from the study revealed a strong connection between the program's espoused comprehensive philosophy and its practice. Embracing diversity of d/Deafness and differentiated instruction were the two strongest themes that resonated from the participants. Teacher candidates displayed outstanding commitment to the profession and strong proficiency in practice, within the first two pillars. Program improvement was most needed in navigating the public school system.
\end{abstract}




\section{CHAPTER ONE INTRODUCTION}

As of 2012, 30\% (15 out of 50) of states within the U.S. reportedly have no existing deaf education teacher preparation programs (Deaf Education Teacher Preparation Programs). Since that time, the state of Michigan no longer has a deaf education teacher preparation program (Michigan State University College of Education, n.d.). However, Connecticut has reportedly joined the ranks of states with deaf education teacher preparation programs (Lenihan, 2010; Paterson \& Cole, 2010). With those changes considered, the number of states with deaf education teacher preparation program remained constant. Additionally, U.S. territories, Guam and American Samoa, the Associate Free State of Puerto Rico, and the U.S. Commonwealth of Northern Marianas Islands reportedly have no deaf education teacher preparation programs (Deaf Education Teacher Preparation Programs, 2012).

Although students who are d/Deaf or hard of hearing (d/DHH) account for only $1.2 \%$ of the total population of students with disabilities in U.S. public schools (U.S. Department of Education, 2012), there exists a critical shortage of teachers of students who are d/Deaf or hard of hearing (d/DHH) in the United States (American Association for Employment in Education, 2008; U.S. Department of Education, 2012). The term $\mathrm{d} /$ Deaf is used because the small "d" deaf refers to audiometrically defined deafness (Trezek, Wang, \& Paul, 2010), whereas the capital "D" Deaf refers to culturally defined deafness (Paul \& Whitelaw, 2011). This critical shortage of U.S. teachers to educate students who are d/DHH has been long-standing (Johnson, 2004). It is imperative that 
states with teacher preparation education of the $\mathrm{d} /$ Deaf and hard of hearing programs work to close the gap between the shortage of deaf educators and the need for deaf educators in public school systems across the country.

Regrettably, the number of deaf education teacher preparation programs continues to decline (Johnson, 2013). Dolman (2008) identified a 17\% decrease in deaf education teacher preparation programs between 1986 and 2006. Furthermore, in a study of deaf education teacher preparation faculty, Benedict, Johnson, and Antia (2011) uncovered three deaf education programs in the process of closing their doors and an additional seven programs at-risk of closing. If those programs indeed close, the Midwest will be hit the hardest. Only nine of the 15 programs in existence would remain, thus resulting in “a $40 \%$ decrease in the region's capacity to prepare teachers of deaf and hard of hearing students" (p. 7).

Program sustainability is further complicated by the scarcity of doctoral-level graduates to replace full-time, retiring, tenured faculty who prepare future teachers of students who are d/DHH (Benedict et al., 2011; LaSasso \& Wilson, 2000). While 24 faculty in deaf education teacher preparation programs were expected to retire in 2013 (Benedict et al., 2011), it was expected that many of those positions would go unfilled. Benedict et al. (2011) reported that fewer than two new graduates of doctoral programs were hired as faculty in deaf education teacher preparation programs annually.

In addition to the declining number of deaf education teacher preparation programs (Johnson, 2013) and the potential challenges facing universities to meet the demand for faculty (Benedict et al., 2011), there is a unique complexity to deaf education teacher preparation programs. This complexity, which may not be found in other types of 
teacher preparation programs, is focused on a single, yet diverse conceptcommunication philosophy. The national approval organization specific to deaf education is the national Council on Education of the Deaf (CED) (Council on Education of the Deaf, 2013). One essential element for CED national approval is that teacher preparation programs design their curriculum to meet approved standards and align these standards with their espoused philosophy.

The three communication philosophy options as identified by CED were auditoryoral, bilingual-bicultural, and comprehensive (CED, 2013). These programs differ in their communication approach to training teacher candidates which results in different outcomes. Teacher candidates leave programs prepared to teach students who are $\mathrm{d} / \mathrm{DHH}$ based on the communication methodology used by the program. So with the dwindling number of programs, there is also the factor of communication philosophy that sets programs apart from each other.

Although the outlook for deaf education teacher preparation programs appears bleak (Benedict et al., 2011; Johnson, 2013), Dolman (2010) noted a positive trend. Enrollment in such programs showed remarkable stability across nearly two decades from 1973-2009 when compared with the estimated number of students who were d/DHH in U. S. schools. Although encouraging, the critical need for teachers of learners who are d/DHH remains (U.S. Department of Education, 2012).

With deaf education falling within an area of low incidence and subsequently often lower enrollment in university teacher preparation programs, deaf education faculty are challenged with the need to "constantly demonstrate that their value is deserving of university support” (Lenihan, 2010, p. 123). Although Lenihan's (2010) quote was 
specific to teacher preparation programs focused on listening and spoken language, the number of declining and at-risk deaf education teacher preparation programs throughout the U.S. demonstrates this challenge is wide-spread (Benedict et al., 2011). Unfortunately, it will be the learners who are $\mathrm{d} / \mathrm{DHH}$ and their families, the public school systems who serve the majority of students who are d/DHH, and ultimately, the greater community who will pay the price for not filling this need within U.S. schools.

\section{Problem Statement}

The literature is riddled with the challenges facing deaf education teacher preparation programs (Benedict et al., 2011; Dolman, 2010; Johnson, 2013; Lenihan, 2010) and the critical need for teachers of students who are d/DHH has been well established (Benedict et al., 2011; Deaf Education Teacher Preparation Programs, 2012; Dolman, 2008; Johnson, 2013; U.S. Department of Education, 2012). Attention to the plight of deaf education has resulted in a push for change in deaf education teacher preparation programs (Johnson, 2004, 2013; Lenihan, 2010). Advances in hearing technologies and other technologies, early identification of hearing loss, and early intervention have also sparked the need for change (Lenihan, 2010; Marvelli, 2010).

Universities have attempted to meet the need for change in deaf education teacher preparation programs through a variety of avenues. In California, the University of San Diego embedded a deaf education component into another existing education program (Humphries \& Allen, 2008). The state of Michigan invited universities to partner with them in a collaborative effort to prepare deaf educators through online learning opportunities (Johnson, 2013). And the University of Hartford in Connecticut responded 
by adding a new program that prepares teachers to develop the listening and spoken language skills of the students they serve (Paterson \& Cole, 2010).

Researchers have identified challenges and proposed solutions (Humphries \& Allen, 2008; Johnson, 2004, 2013; Lenihan, 2010; Paterson \& Cole, 2010). Yet, little is known about the success of education of the d/Deaf and hard of hearing teacher preparations programs that exist. Humphries and Allen (2008) conducted one of few studies on deaf education teacher preparation program functioning. They began documenting the effects on the graduates and schools they served through the University of California - San Diego's ASL and English bilingual program. Early findings have resulted in some curricular adjustments. There was also notable acknowledgement of student teachers' competence by school teachers and administrators.

While Humphries and Allen (2008) studied a program using a bilingual-bicultural approach, Winn (2007) studied Australia's Griffith University's undergraduate program that prepares teachers of students who are d/DHH using a comprehensive philosophy. In part, Winn gathered data from 10 students on their perceived training needs for teaching students who were d/DHH. Although Winn's (2007) study provided a thorough program description, he stopped short of using explicit findings from which to draw a data supported conclusion. There exists a gap in the literature on the functioning and success of deaf education teacher preparation programs.

According to Deaf Education Teacher Preparation Programs (2012) there were 64 deaf education teacher preparation programs in existence in the U.S. Although there was a change in which states no longer had deaf education teacher preparation programs, the number of states without programs remained the same (Lenihan, 2010; Michigan State 
University College of Education, n.d.; Paterson \& Cole, 2010). Fifteen states were reportedly without deaf education teacher preparation programs.

Thirty-eight of the existing 64 deaf education teacher preparation programs reported using a comprehensive approach or philosophy (Deaf Education Teacher Preparation Programs, 2012). Auditory-oral and bilingual-bicultural programs were nearly split with 10 and eight respectively. Programs that listed listening and spoken language were included within the auditory-oral program count. The 10 remaining programs listed their philosophy with some other label indicating some combination of approaches, an all-inclusive approach, or no response (Deaf Education Teacher Preparation Programs, 2012).

A majority of U.S. deaf education teacher preparation programs self-identify as preparing future teachers using a comprehensive philosophy (Deaf Education Teacher Preparation Programs (2012). There is a gap in the literature about how comprehensive programs function. Even less is known about the functioning of these programs and how they prepare deaf education teacher candidates while simultaneously providing on-site services to learners who are $\mathrm{d} / \mathrm{DHH}$ within their program.

\section{Research Purpose}

A Midwest's comprehensive, graduate deaf education teacher preparation program and its teacher candidates will be the focus of a phenomenological case study. From this point forward, this deaf education teacher preparation program will be referred to as Midwest University. Even though a majority of U.S. deaf education teacher preparation programs self-identify as comprehensive, this is the only program in the state in which this program resides that espouses a comprehensive philosophy. In addition, in 
relation to this Midwest state, three out of the four primary bordering states have no deaf education teacher preparation programs.

As previously established, the Midwest will be hit the hardest if the deaf education teacher preparation programs at risk of closing actually close (Bendict et al., 2011). This reduction of programs would be an additional strain on the region's capacity to prepare these educators. Finally, little is known about how comprehensive programs function to prepare future teachers of students who are $\mathrm{d} / \mathrm{DHH}$ while simultaneously filling a community need. Midwest University prepares deaf education teacher candidates while also providing direct services to young children who are d/DHH.

The intent of this phenomenological case study will be to provide a detailed description of Midwest University's program and its teacher candidates. Specifically, this study will explore the essence of the central phenomenon, empowered and enabled learning (Marquardt, 2011) as it relates to the program's preparation of teacher candidates. At this stage in the research, empowered and enabled learning will be generally defined by the three overarching professional educator frames adopted and approved by Missouri's Department of Elementary and Secondary Education (MODESE): (a) commitment to the profession, (b) proficiency in practice, and (c) learning impact (Missouri's Department of Elementary and Secondary Education, MO-DESE, 2011).

According to Marquardt's (2011) systems learning framework, the teacher candidates are not only the product of Midwest University, but, also the customers of this organization. In fact, they are the front-line customers and future employees of organizations, often the U. S. public school system, to educate learners who are d/DHH. 
The teacher candidates are paramount to the organization's success as well as to filling the critical need that exists for teachers of students who are $\mathrm{d} / \mathrm{DHH}$ in public schools across the U.S. (American Association for Employment in Education, 2008; U.S. Department of Education, 2012). Marquardt's (2011) learning organization people subsystem will guide the investigation and frame the research questions.

\section{Research Questions}

Within the context of this study, the following research questions were addressed:

1. How does the program empower and enable teacher candidates to learn commitment to the deaf education profession?

2. How does the program empower and enable teacher candidates to learn proficiency in practice with students who are d/Deaf or hard of hearing?

3. How does the program empower and enable teacher candidates to create learning impact in students who are d/Deaf or hard of hearing?

4. How could the program improve to empower and enable the learning of teacher candidates who are prepared to teach students who are d/Deaf or hard of hearing?

\section{Conceptual Framework Guiding Study}

Learning is critical to organizational viability. In fact, learning is the driving force behind all learning organizations (Marquardt, 2011; Senge, Roberts, Ross, Smith, Kleiner, 1994; Senge, 1990). There is agreement that learning must occur rapidly to keep up with the ever growing and changing society (Marquardt, 2011; Senge, 1990). If learning is fundamental, then value and commitment to learning must be a part of the organization's culture, both in word and action (Belasen, 2000; Marquardt, 2011; Schein, 2010). Yet, organizational learning will simply not occur without people (Marquardt, 
2011). In this ever changing, knowledge driven economy, organizations need people more than ever (Bolman \& Deal, 2008; Marquardt, 2011).

The heart of a learning organization is evidenced by its use of human capital (Marquardt, 2011). Marquardt (2011) believed one of the most fundamental organizational challenges was, "how to attract, deploy, develop, adapt, and retain it [human capital] better than anyone else" (p. 93). Learning organization researchers (Belasen, 2000; Marquardt, 2011; Senge, 2006; Senge et al., 1994) and others (Bolman \& Deal, 2008; Kouzes \& Posner, 2010, 2011; Maslow, 1943/2011; McGregor, 1957/2011) understood that the answer lay within their approach with people.

Learning organizations view people through a human resource lens (Bolman \& Deal, 2008; Marquardt, 2011). These organizations respond to the needs of their people (Belasen, 2000; Bolman \& Deal, 2008; Maslow, 1943/2011) and invest in them (Bolman \& Deal, 2008; Marquardt, 2011). Learning organizations build human performance capability with a balance for meeting both individual and organizational needs (Marquardt, 2011). A good fit between the people and the organization yields benefits to both (Bolman \& Deal, 2008).

As Marquardt (2011) stated, "organizations must learn even faster and adapt more seamlessly to changes in the environment or they simply will not survive" (p. vii). During a time in which exists a critical shortage of educators of learners who are d/DHH in public schools within the United States (U.S. Department of Education, 2012), deaf education teacher preparation programs are struggling to survive (Benedict et al., 2011; Dolman, 2008; Johnson, 2013). Marquardt espoused that learning organizations require organizational investment in the development of the following five subsystems: (a) 
learning dynamics, (b) organizational transformation, (c) empowering people, (d) knowledge management, and (e) application of technology. Though Marquardt's model is comprehensive and could guide exploration of multiple aspects of organizational learning, this study will focus on his people subsystem model. Particularly, this study will explore empowered and enabled learning of Midwest University's deaf education teacher candidates (Marquardt, 2011).

Marquardt (2011) and others (Belasen, 2000; Bolman \& Deal, 2008; Kouzes \& Posner, 2010) agreed on the importance of human relationships in organizations and emphasized that leadership should include a focus on people empowerment. Although similarities exist, there are also inconsistencies in the way researchers define empowerment. Bolman and Deal (2008) indicated that employees are empowered when an organization invests in their development, keeps them informed, balances engagement and autonomy, and infuses their work with meaning.

In Marquardt's (2011) people subsystem, Marquardt equated empowerment with people having "necessary resources at their disposal" (p. 93). Marquardt (2011) also supported an organizational paradigm shift whereby leaders and managers act as stewards, moving away from traditional forms of control toward empowerment of employees. While Marquardt (2011) and others (Belasen, 2000; Bolman \& Deal, 2008; Kouzes \& Posner, 2010) focused on leaders or managers empowering their followers, Marquardt also purported internal and external stakeholders must be empowered to learn.

Marquardt (2011) further stipulated that organizational learning was dependent on people being both "empowered and enabled to learn" (p. 26); one without the other was not enough. According to Marquardt, enabled learning is about people having the 
knowledge necessary to use resources effectively. He viewed enabling learning as the most essential responsibility of managers. Marquardt also recognized that people in all subsystem groups influence the learning of others. Aligned with Marquardt's model, deaf education teacher preparation faculty bear some responsibility for empowering and enabling graduate teacher candidates to learn.

Teacher preparation programs are not isolated entities; but, rather intricately intertwined with stakeholders both inside and outside of the organization. As espoused by Marquardt (2011) all people need to be empowered and enabled to learn for organizational learning to occur. It is Marquardt's systems learning organization's people subsystem that will guide the research. Midwest University and its people, namely, teacher candidates, will be explored through a central phenomenon of study.

\section{Design and Methods}

Qualitative inquiry maximizes the richness of collected data while exploring the essence of a central phenomenon (Creswell, 2009). This researcher will study Midwest University's comprehensive, deaf education teacher preparation's graduate program and its people through a phenomenological case study. In contrast to Creswell's view of a case study as a qualitative, stand-alone, strategy of inquiry, this researcher embraces Savin-Baden and Major's (2013) definition in which case study is not synonymous with methodology. Savin-Baden and Major argued that researchers often embed a specific methodology, such as phenomenology, within their case study even if it is not made explicit by the researcher. This case study was bounded by one graduate deaf education teacher preparation case, Midwest University, which espouses a comprehensive philosophy. 
For this research, a phenomenon in the bounded case of a graduate, comprehensive, deaf education teacher preparation was explored through a qualitative, phenomenological case study (Savin-Baden \& Major, 2013). This study was also be bounded by time, a three-year academic calendar year from fall 2011 through spring 2014. The researcher explored an understanding of a central phenomenon as it related to teacher candidates. The phenomenon studied, empowered and enabled learning, was drawn from Marquardt's (2011) learning organization people subsystem.

Qualitative research design provided the most thick and rich (Hatch, 2002) data for an in-depth exploration of the phenomenon within the context of a single deaf education teacher preparation program (Savin-Baden \& Major, 2013). A variety of groups of individuals were included in the study. In addition, a variety of data collection methods were employed to increase understanding of the case (Creswell, 2009): (a) interviews, (b) surveys, and (c) documents. Aligned with the researcher's primarily postpositivist worldview perspective (Creswell, 2009; Hatch, 2002), the researcher used typological analysis to provide more structure to data analysis. This analysis allowed for deductive and inductive reasoning approaches resulting in emerging themes of the phenomenon studied (Hatch, 2002).

\section{Setting}

A brief history of the setting was included to provide a timeline on Midwest University's program development, faculty configuration, and program model which included service provisions for young children who are $\mathrm{d} / \mathrm{DHH}$ and their families. This section included a preview of the current setting. The current setting was further outlined within the methods and findings sections of this paper. 
In 1982, the Coordinating Board for Higher Education approved the addition of a master's degree in the department of Communication Disorders with an emphasis in Education of the Deaf and Hard of Hearing at Midwest University (Midwest University web-site A, 2013). Although the program offers both an undergraduate and graduate degree, initial certification occurs at the graduate level. Six years later, the university received approval from deaf education teacher preparation program's national approval body, CED. Midwest University embraces a comprehensive philosophy as identified by CED (CED, 2013). Teacher candidates gain experiences in both oral and manual communication methodologies.

While all deaf education teacher preparation programs must comply with state certification standards, adhering to national certification standards is optional (Johnson, 2013). Johnson (2013) reported that only half of the deaf education teacher preparation programs were designed to fulfill national certification standards set forth by the Council on Education of the Deaf (CED) and the Council on Exceptional Children (CEC) in addition to their state standards. Midwest University was one of the deaf education teacher preparation programs that met this higher standard.

In 1989, Midwest University received two personnel preparation grants from the U.S. Department of Education (Midwest University '88-'89 Accomplishments, 1989). In part, the grants supported the addition of two personnel lines to enhance deaf education teacher candidate preparation in practicum experiences with young children who are $\mathrm{d} /$ DHH and their families while simultaneously serving the community (Midwest University documents A \& B, 1989). The two personnel lines allowed Midwest University to hire two deaf educators with master's degrees, which expanded this deaf 
education teacher program to four full-time positions: (a) two Ph.D. academic faculty, and (b) two masters-level laboratory supervisors. In 2007, the laboratory supervisor positions became full-time clinical faculty lines.

In 1990, Midwest University started a site-based preschool program for preschoolers who are d/DHH, ages 3-6 years old. This program was housed within the department's speech, language, and hearing clinic. At the time of its inception, one of the supervisor's was the preschool classroom teacher with a combined role as a university supervisor of undergraduate and graduate teacher candidates who were assigned to the preschool for practicum. This deaf educator also served as a student teaching preceptor and taught college-level sign language classes. The preschool program followed the university's semester calendar. Preschool services were provided five days a week for three hours each day. Related services, speech and audiology were also provided.

At the time of this study, the family-centered preschool program for children who are d/DHH operated four days weekly for three hours each day. Regardless of mode of communication, use or non-use of amplification, and the existence of additional disabilities, all preschoolers, ages 3-6, who are d/DHH were welcome. Therefore, during any given semester, the preschoolers enrolled in the program formed an eclectic group.

Some preschoolers' primary mode of communication was spoken language while others communicate through sign language, exclusively using American Sign Language or more frequently, conceptual signs in English word order. Still others use a combination of speech and sign. An overwhelming majority of the children served in the preschool use assistive listening technology, either cochlear implants, hearing aids, or a 
combination of the two. Children enrolled in the program have included children who only present with hearing loss up to and including children with multiple disabilities.

In this language-intensive preschool environment, the children were immersed in language through multiple modalities: (a) spoken, (b) signed, and (c) written. There were expectations that all of the preschoolers will become "independent and literate" (Wang, Engler, \& Oetting, 2014, p. 36). Development of the whole child is emphasized. Therefore, in addition to language, literacy, and independence, the areas of academics, speech, auditory, social, and self-advocacy were also integrated within the program. Individualized speech therapy and audiological services were also provided within the clinic by speech graduate clinicians and audiology doctoral students. Teacher candidates in the deaf education teacher preparation program complete three consecutive practicum experiences with increasing difficulty and expectations within the preschool setting.

Initially, the second master-level supervisor served infants and toddlers who were $\mathrm{d} / \mathrm{DHH}$, ages birth to 3 years, and their families. The parent infant program provided both home-based and on-site services. On-site services included individualized familydriven services in a home simulation lab which was designed as a small apartment to provide a home-like environment for service provision. The lab was used when distance prohibited the supervisor from traveling to the family's home.

A weekly toddler group for toddlers who were d/DHH and their families and, at times, included Deaf adults and peers who were hearing, was also held on-site. This supervisor also taught college level sign language courses as a part of her responsibilities. In addition, this supervisor periodically served as a university supervisor of 
undergraduate and graduate teacher candidates enrolled in practicum and as a student teaching preceptor for student teaching placements in parent-infant as needed.

Since its inception, Midwest University's deaf education teacher preparation program typically functioned with four full-time positions. However, between 2007 and 2008, two full-time professors retired and one assistant professor was hired in fall 2008. In 2009, an accelerated master's option was added to Midwest University. The accelerated master's option was designed to allow this deaf education teacher preparation program to function with one full-time academic professor and two full-time clinical faculty members with additional per course faculty support. To date, Midwest University functions with one full-time academic faculty member and two full-time clinical faculty members with the addition of per course faculty support.

The decrease in faculty at Midwest University from four full-time lines to three full-time lines has created a shift in teaching loads and program responsibilities. One change that began in spring 2011 that may have ramifications, was an ending of parentinfant services for infants and toddlers who are $\mathrm{d} / \mathrm{DHH}$ and their families. Several factors attributed to the dissolution of parent-infant services, albeit, this disruption in service provision is considered temporary.

Following were the basic factors that lead to the temporary dissolution of parentinfant services at this deaf education teacher preparation program as identified by Midwest University deaf education faculty. First, the clinical faculty member who provided these services was shifted into fulfilling more of the graduate teaching load. This graduate coursework was unable to be absorbed by the one academic faculty member hired after the retirement of two academic faculty members. 
Second, there was a temporary lull in families requesting parent-infant services in this Midwest region, which made it difficult to justify the need for this per course hire to department and college administrators. Anecdotal evidence of an increasing number of phone calls requesting parent-infant services showed that this lull was short-lived. Furthermore, there were challenges with justifying the hiring of per course faculty each semester to fulfill a role that did not consistently generate student enrollment dollars.

The disruption in parent-infant service delivery at Midwest University also impacted practicum preparation experiences for teacher candidates at Midwest University. Primarily, teacher candidates were no longer able to complete a practicum experience in parent-infant at Midwest University and no longer were teacher candidates who had an interest in parent-infant able to complete a student teaching assignment in this area at Midwest University. To date, the most recent eight-week student teaching placement experience in the parent-infant program at Midwest University occurred in spring 2009. However, during the three-year span of this study, two teacher candidates who had strong interest in early intervention were provided with mini-internships in parent-infant. Each teacher candidate completed a week-long, supervised mini-internship with young children who were $\mathrm{d} / \mathrm{DHH}$ and their families. This was a collaborative opportunity between Midwest University and a School for the Deaf in another state. Midwest University's Communication Sciences and Disorders (formerly Communication Disorders) Department's graduate, comprehensive deaf education teacher preparation program was approved by CED in 1989. At the time of this study, the program functioned with one full-time academic faculty, two full-time clinical faculty, and per course faculty. In conjunction with the preparation of teacher candidates, 
Midwest University has been providing services to young children who are $\mathrm{d} / \mathrm{DHH}$ and their families for nearly 25 years.

Due to a variety of factors, at the time of this study, the program no longer served infants and toddlers who are $\mathrm{d} / \mathrm{DHH}$ and their families. The on-site preschool program is open to all children who are $\mathrm{d} / \mathrm{DHH}$ regardless of mode of communication, use of amplification technology, or the presence of additional disabilities. This languageintensive, literacy-focused environment also serves as a practicum site for the teacher candidates across three consecutive semesters.

\section{Assumptions}

By its very nature, "qualitative work starts with the assumption that social settings are unique, dynamic, and complex" (Hatch, 2002, p. 9) and researchers approach study of the whole rather than pieces. Qualitative methods provide for the collection of rich data by exploring the essence of a phenomenon. Methods emphasize natural contexts and focus on representing the voices of the people studied.

Qualitative research design also includes challenges (Creswell, 2009; Frankel \& Wallen, 2009; Hatch, 2002; Patton, 1999; Savin-Baden \& Majors, 2013). "In qualitative study, much depends on the perspective of the researcher"' (Frankel \& Wallen, 2009, p. 453). And "all researchers have...biases" (Frankel \& Wallen, 2009, p. 453). Researchers need to be upfront about their beliefs and position on relevant issues of their study (Hatch, 2002). Researcher bias and assumptions were addressed below and were further explored within Chapter Three.

The researcher of this study has been teaching within this deaf education teacher preparation program for 22 years. Without a doubt, this researcher has a vested interest 
in the program, in the graduate teacher candidates whom she serves, and in the outcomes of the ultimate customers, those young children who are $\mathrm{d} / \mathrm{DHH}$ and their families. Furthermore, this researcher was the supervisor and teacher within the preschool program for 12 years; however, she has not served in this capacity for the past decade. In addition, the researcher has the assumption that participants, including those who are enrolled in one of the researcher's course(s), shared openly and honestly. There was also the assumption that the outcomes reported by parents of the impact of the clinic preschool program on their children were influenced by the program's teacher candidates.

\section{Definition of Key Terms}

The following categorical terminologies were critical for understanding this study: (a) description of deafness, (b) communication philosophies, and (c) empowered and enabled learning phenomenon. Each category included a brief introduction of why the terms were grouped within that category. Next each specific term was defined.

\section{Description of Deafness}

Deafness has been defined in a multitude of ways (Paul \& Jackson, 1993; Paul \& Whitelaw, 2011; Trezek et al., 2010). Perceptions of deafness often influence the terminology used to define deafness and tend to center around two distinct paradigms, one in which deafness is viewed from a medical or clinical perspective and the other in which deafness is viewed from a cultural perspective (Paul \& Jackson, 1993). A medical or clinical perspective often defines deafness in terms of lack or deficit; thereby, focusing on degree of hearing loss and deafness as a disability. From a cultural perspective, deafness is viewed as a natural occurrence that should be embraced, rather than fixed (Paul \& Jackson, 1993; Paul \& Whitelaw, 2011). 
Deaf. An individual who self-identifies as capital "D" Deaf embraces deafness and indicates membership to Deaf Culture (Paul \& Whitelaw, 2011). The use of American Sign Language is a significant identifier of somebody belonging to this cultural group (Paul \& Jackson, 1993). There is a strong sense of pride and a longing to preserve the Deaf Community. Although individuals who are Deaf may often have a severe to profound bilateral hearing loss (Paul \& Whitelaw, 2011), this is not always the case. One may self-identify as Deaf and have more residual hearing than somebody who presents as audiometrically deaf.

deaf. Individuals who are deaf have a bilateral hearing loss which audiometrically places their hearing loss within a severe to profound category (Trezek et al., 2010). A severe category indicates an unaided hearing loss within the 70-90 decibel (dB) hearing level (HL) range. A profound hearing loss falls at a $90 \mathrm{~dB}$ hearing level or greater (Cole \& Flexer, 2011).

Hard of hearing. Audiometrically, individuals who fall within the category of hard of hearing have hearing acuity that is better than a severe level of $70 \mathrm{~dB}$ HL (Trezek et al., 2010). Paul and Jackson (1993) and Paul and Whitelaw (2011) noted that individuals were labeled hard of hearing based on the successful use of their remaining hearing or residual hearing. In addition, as reported in Schow and Nerbonne (2002), individuals who are hard of hearing often communicate through spoken language.

\section{Communication Philosophies}

Three specific communication philosophies identified by the national approval body for education of the deaf, CED, were: auditory-oral, bilingual-bicultural, and comprehensive. Deaf education teacher preparation programs who seek national 
approval were required to state their espoused philosophy and align their philosophy with the required CED and CEC approved standards (CED, 2013). Communication philosophies dictated the methodology of instruction for preparing teacher candidates to teach students who are d/DHH.

Auditory-oral. "Auditory-oral education is designed to help children with hearing loss learn to talk well enough to communicate confidently and accurately solely through the use of speech" (Moog, 2007, p. 131). Auditory-oral programs maximize use of audition through aggressive audiological management such as the proper fitting and use of hearing aid and cochlear implant technologies as well as through the development of listening skills. Speechreading may be emphasized in some programs and deemphasized in others.

Bilingual-bicultural. The bilingual-bicultural approach is more recently referred to as the ASL and English bilingual approach (Benedict \& Sass-Lehrer, 2007). This philosophy takes the educational position that children who are d/DHH should learn two languages. It is believed that these children should learn "their native sign language and the native spoken language of their country" (Benedict \& Sass-Lehrer, 2007, p. 185). In an ASL and English bilingual approach, English is taught as a second language. The goal is to achieve fluency in both languages. This approach may also include the development of spoken English.

Comprehensive. The comprehensive philosophy definition that was used was articulated by the state of Virginia's Radford University as reported in Deaf Education Teacher Preparation Programs (2012). Their comprehensive program was "designed to prepare teachers to serve children and youth with hearing loss regardless of 
communication approach" (Deaf Education Teacher Preparation Programs, 2012).

Therefore, a comprehensive program is focused on meeting the individualized, diverse needs of young children and students who are d/DHH regardless of methodology.

\section{Empowered and Enabled}

As previously stated, similarities and differences exist in the way that empowerment is defined (Belasen, 2000; Bolman \& Deal, 2008; Kouzes \& Posner, 2010; Marquardt, 2011). Marquardt (2011) purported that both empowered and enabled learning was critical to organizational success. His definitions of empowered and enabled learning hinged on people having both knowledge and resources. One without the other was simply not sufficient. Marquardt stated that if people were "empowered but not enabled, they will have the necessary resources at their disposal, but not the knowledge to effectively use them" (p. 93). He further explained that if people were "enabled but not empowered, [they] will have the necessary knowledge, but not the ability to apply it" (p. 93). Marquardt also stressed a paradigm shift from toward empowerment and away from leadership control.

The three professional educator frames adopted by MO-DESE (a) commitment to the profession, (b) proficiency in practice, and (c) learning impact (MO-DESE, 2011) were used in the preliminary working definition of Marquardt's (2011) "empowered and enabled to learn" (p. 26) phenomenon. The standards were created as a guide for public school districts within the state of Missouri. The model is built around the core concept that "educators are caring, reflective practitioners and lifelong learners" (MO-DESE, 2011, p. 3) who meet the needs of every student thus assisting the students "in reaching 
their highest potential no matter the demographics" (p.3). Each of the three identified pillars follows.

Commitment to the profession. Commitment to the profession is rooted in professionalism, lifelong learning, and high expectations reflected in caring teacher candidates who demonstrate the determination to assist every student in reaching their full potential (MO-DESE, 2011).

Proficiency in practice. For this study, practice was be defined as (a) providing meaningful learning opportunities adapted to diverse learners that support development of the whole student: academically, socially, and personally (b) utilizing strategies that promote the development of students' critical thinking, problem solving, and effective communication skills, and (c) creating positive learning environments which promote student engagement, self-motivation, collaboration, and respect for all (MO-DESE, 2011).

Learning Impact. Learning impact was determined through participants’ perception of learning impact on students who are $\mathrm{d} / \mathrm{DHH}$ that the teacher candidates served. Participant perceptions were gathered from deaf education teacher preparation faculty, teacher candidates, and teacher candidates' practicum and student teaching preceptors. Preceptors were public school or School for the Deaf teachers of students who were d/DHH. The preceptors provided model teaching, mentoring, and on-site supervision to program teacher candidates during teacher candidates' off-site placements. In addition, archival survey documents of parents' perception of Midwest University's clinic preschool program's impact on their preschoolers, ages 3-6, who received services 
during this bounded three-year case study timeframe was also included to evaluate learning impact.

\section{Significance of the Research for Leadership Practice}

The significance of this study extends beyond filling a gap in the literature about how a graduate, comprehensive deaf education teacher preparation program functions. This research explores not only a program's functioning, but, its impact on the learners who are d/DHH whom they serve. Furthermore, the possible synergistic outcomes of teacher preparation programs who prepare teacher candidates and successfully serve students who are d/DHH may have far-reaching implications.

This research may open the door to "learningful" (Senge, 1990, p. 8) conversations to inform future educational leadership policies and practices. For example, it may forge new partnerships between higher education institutions and public school programs in the U.S. It may encourage state departments of education who have no deaf education teacher preparation programs to provide tuition support to university bound students. University teacher candidates could, as a part of a scholarship or stipend agreement, return to their state to teach students who are $\mathrm{d} / \mathrm{DHH}$ for a specified number of years. It may spark national funding opportunities that support higher education institutions who meet a dual purpose of preparing teachers in this low incidence area while simultaneously serving students who are d/DHH through provided services. Furthermore, this study may provide insights into empowered and enabled learning (Marquardt, 2011) of teacher candidates which may benefit not only Midwest University, but, other deaf education teacher preparation programs. 


\section{Summary}

A crisis exists in the field of deaf education. Deaf education teacher preparation programs were on the decline (Dolman, 2008; Johnson, 2013) and others were at risk of closing (Benedict et al., 2011). At the same time, educators of students who are d/DHH were in high demand within the nation (American Association for Employment in Education, 2008; U.S. Department of Education, 2012). Yet little is known about deaf education teacher preparation programs that exist and even less is known about how these programs prepare teacher candidates while simultaneously serving young children who are $\mathrm{d} / \mathrm{DHH}$. This researcher studied a deaf education teacher preparation program that embraced a comprehensive philosophy through a qualitative, phenomenological case study. Marquardt's (2011) people subsystem guided the investigation. 


\section{CHAPTER TWO}

\section{REVIEW OF THE LITERATURE}

Estimates of hearing loss within the U.S. suggest that hearing loss is the most frequently occurring birth defect (Flexer \& Madell, 2008, p. xix). Yet, "deafness is a condition that remains a mystery to most members of the general hearing community" (Moores, 2001, p. 1). For many, deafness is viewed through a lens of deficit, something to be prevented, fixed, or cured (Crittenden, 1993; Moores, 2001; Paul \& Whitelaw, 2011). This view of deafness is often referred to as a medical (Moores, 2001; Paul \& Jackson, 1993) or pathological perspective (Moores, 2001). Its focus, "normalization" (Moores, 2001, p. 250), places an emphasis on speech and spoken language development, thus more closely mirroring a child with hearing for functioning within "the surrounding culture's language" (Spencer \& Marschark, 2010, p. 53). Through this lens, "deafness is seen as a disability" (Schirmer, 2001, p. 81).

Another model of deafness exists, one rooted within many in the Deaf Community and shared by an increasing number of individuals who are hearing. For these individuals, deafness is viewed from a sociocultural perspective or model (Moores, 2001; Schirmer, 2001). Rather than deficiency (Moores, 2001) or disability (Schirmer, 2001), this model embraces deafness as a difference (Moores, 2001; Paul \& Jackson, 1993; Paul \& Whitelaw, 2011). "Deafness is seen as a linguistic and ethnic minority culture" (Schirmer, 2001, p. 81). American Sign Language, a recognized true language (Marschark, Lang, \& Albertini, 2002), is valued by proponents of this model (Crittenden, 1993). 
According to the National Institute on Deafness and Other Communication Disorders (NIDCD), for every 1,000 U.S. births, approximately 2-3 babies enter the world $\mathrm{d} /$ Deaf or hard of hearing $(\mathrm{d} / \mathrm{DHH})$ (National Institute on Deafness and Other Communication Disorders, 2010). And greater than $90 \%$ of these children were born to parents who are hearing (Marschark et al., 2002). That percentage has been estimated as high as 96\% (Mitchell \& Karchmer, 2004). Often these parents have no prior knowledge or experience with deafness. From a linguistic minority culture perspective, these parents are not members (Marschark et al., 2002).

The two divergent medical and sociocultural models perspectives have resulted in controversy and tension (Paul \& Whitelaw, 2011). Paul and Whitelaw believed there was value in both models and that both should be respected. Moores (2001) conceptualized the sociocultural model of deafness not as two different worlds—-"a deaf world and a hearing world - but one world in which deaf and hearing people exist and interact on a daily basis" (p. 1). Regardless of one's worldview or perspective on deafness, complexities and challenges exist for individuals who are $\mathrm{d} / \mathrm{DHH}$ living in a hearing society.

Individuals who are d/Deaf continue to face obstacles in a variety of areas: (a) communication, (b) education, and (c) social (Anderson \& Matkin, 1991/2007; Moores, 2001; Tye-Murray, 2009). For some, including adults with late-onset hearing loss, every day communication and conversations may be difficult across settings and require significant effort (Stenross, 1999; Tye-Murray, 2009). Young children who are d/DHH may also experience delays in speech and language (Tye-Murray, 2009), especially in the area of spoken language (Lederberg, Schick, \& Spencer, 2012). Furthermore, 
consequences of hearing loss are not limited to individuals with a seemingly, substantial hearing loss. Negative educational ramifications of hearing loss have been documented with individuals who have unilateral hearing loss, hearing loss that was believed to be relatively inconsequential in the past (Bess, \& Tharpe, 1984).

Children with unilateral hearing loss were found to be at risk academically (Anderson \& Matkin, 1991/2007; Bess \& Tharpe, 1984). They were nearly 10 times more likely to fail a grade in school when compared against the school population as a whole (Anderson \& Matkin, 1991/2007; Bess \& Tharpe, 1984; Northern \& Downs, 1991). Bess and Tharpe (1984) conducted a study of 60 students in Nashville, Tennessee who had single-sided hearing loss measuring at $45 \mathrm{db} \mathrm{HL}$ or greater and hearing acuity no poorer than $15 \mathrm{~dB} H L$ in the unaffected ear. Of those 60 students, $35 \%$ failed at least one grade in school compared to a 3.5\% general failure rate of students from kindergarten through sixth grade in the Nashville public school system. Likewise, milder forms of bilateral hearing loss, also previously thought to be non-problematic, may indeed pose potential risk to children (Spencer \& Marschark, 2010). In essence, there may be no such thing as an insignificant hearing loss, at least where children are concerned (Flexer, 1994; Welling \& Ukstins, 2015).

The literature review provided the backdrop for this particular study. First, the various levels of hearing acuity were defined with potential ramifications of each level of hearing acuity explained. Next, there was a section about challenges as well as advances related to the education of students who are $\mathrm{d} / \mathrm{DHH}$. The final section focused on educating deaf education teacher candidates. 


\section{Audiometrically Defined Hearing Acuity}

"Hearing loss can be defined medically, educationally, and culturally" (Schirmer, 2001, p. 3). Medically, hearing loss is defined within categories according to the degree or severity of hearing loss. Degree of hearing loss is typically determined by the puretone average (PTA) thresholds across the following speech frequencies for each ear as measured in hertz (HZ): $500 \mathrm{HZ}, 1000 \mathrm{HZ}$, and $2000 \mathrm{HZ}$ (Tye-Murray, 2009). A threshold for a given frequency is determined by the softest intensity of sound a person is able to detect about $50 \%$ of the time (Cole \& Flexer, 2011). Thresholds may be different for different frequencies and ears; therefore, an individual's hearing loss may include more than one category or level of hearing sensitivity (Welling \& Ukstins, 2015).

Most researchers are in agreement that a hearing sensitivity of 25 decibels hearing level $(25 \mathrm{db} \mathrm{HL})$ or better is considered to be at the upper limits of the normal range of hearing sensitivity for adults (Flexer, 1994; Madell \& Flexer, 2008; Northern \& Downs, 1991; Trezek et al., 2010; Tye-Murray, 2009; Welling \& Ukstins, 2015). Some researchers believe the $25 \mathrm{~dB}$ HL cut off is inadequate for children (Flexer, 1994; Madell \& Flexer, 2008; Northern \& Downs, 1991; Welling \& Ukstins, 2015). A slight hearing loss between $15 \mathrm{~dB}$ and $25 \mathrm{~dB}$ may be of significance to young children (Flexer, 1994; Madell \& Flexer, 2008; Northern \& Downs, 1991; Welling \& Ukstins, 2015).

Spencer and Marshark (2010) reported that research has shown that slight or minimal hearing loss may impact academic achievement. Paul and Whitelaw (2011) underscored that research has not shown the relevance for the hearing acuity category distinctions between slight hearing loss and mild hearing loss. Paul and Whitelaw further 
purported that limited research existed "on the relationship between a specific category of hearing loss... and educational achievement" (p. 6).

The following section delineated the different levels of hearing acuity with an emphasis on children who are d/DHH. In addition, potential ramifications of hearing loss were addressed. While variations exist in the way researchers label the decibel levels within each hearing acuity category (Cole \& Flexer, 2011; Northern \& Downs, 1991; Paul \& Whitelaw, 2011; Tye-Murray, 2009), Cole and Flexer's (2011) acuity levels were adopted for the purposes of this study. The content following each heading was compiled from a variety of resources.

\section{Normal Hearing Acuity: 0 - 15 dB HL for Children}

A child who falls within the normal range of hearing acuity should have access to all the sounds of speech. There are no expected negative ramifications due to this level of hearing acuity (Northern \& Downs, 1991). Some indicate that normal hearing sensitivity range begins at a negative $10 \mathrm{~dB}$ HL (-10 dB HL) (Paul \& Whitelaw, 2011). Audiometric zero, written as $0 \mathrm{~dB}$ HL denotes average normal hearing sensitivity; however, individuals may have hearing that is better than $0 \mathrm{~dB} \mathrm{HL}$, thus falling at a negative decibel hearing level (Welling \& Ukstins, 2015).

\section{Slight or Minimal Hearing Loss: 15 - 25 dB HL for Children}

Spencer and Marshark (2010) indicated that students with a slight or minimal hearing loss are often not recognized or counted as students who are d/DHH. Teachers and even the students themselves may be unaware of the impact of this degree of hearing loss (Spencer \& Marshark, 2010). As Welling and Ukstins (2015) explained, "hearing threshold levels consistently within the slight or minimal hearing loss range, particularly 
where children are concerned, can have an adverse effect on communication development and performance" (p. 100). This may include an impact on speech, language, and social development (Anderson \& Matkin, 1991/2007). It may also affect the academic achievement of students (Marschark et al., 2002). In essence, a slight or minimal hearing loss, also referred to as a borderline hearing loss (Tye-Murray, 2009), may have much more effect than the name implies (Flexer, 1994; Welling \& Ukstins, 2015). Flexer (1994) suggested not using the term, minimal, when referring to this degree of hearing loss as it could be construed as inconsequential.

Children falling within this category may have difficulty hearing voiceless consonant sounds (Northern \& Downs, 1991). Challenge with access to voiceless sounds, such as [s, t], could result in difficulties with language concepts such as plurals, possessives, and past tense verbs (Welling \& Ukstins, 2015). Furthermore, this level of hearing loss may be problematic for hearing in the presence of background noise and hearing softer speech or speech at a distance (Anderson \& Matkin, 1991/2007). Anderson and Matkin (1991/2007) also reported that the listener with a minimal hearing loss may miss subtle conversational signals and experience difficulty understanding quickly paced conversations which may affect the child socially. There may also be increased fatigue due to an increased effort for listening.

Incidental learning may also be one of the reasons for a shift to include this slight or minimal hearing acuity range for children. Incidental learning occurs naturally, without direct instruction, when children interact in their environment for which they have awareness (Welling \& Ukstins, 2015) and access. In this particular circumstance, access is referring to auditory access. For children, a slight hearing loss may impede 
optimal listening conditions for incidental learning to happen spontaneously (Welling \& Ukstins, 2015).

\section{Mild Hearing Loss: 25 - 40 dB HL}

Flexer (1994) suggested avoiding use of not only "minimal" but also "mild" hearing loss as the terms seemed to "imply without consequence" (p. 18). Even with amplification, children with a mild degree of hearing loss miss some speech which could lead to misunderstandings and learning difficulty (Anderson \& Matkin, 1991/2007). Difficulty with some early literacy skills such as sound-to-letter associations may make reading more difficult. Class discussions are particularly challenging. Children at the higher end of this hearing acuity range, $40 \mathrm{~dB}$ HL, may miss up to $50 \%$ of classroom discussions (Anderson \& Matkin, 1991/2007).

Anderson and Matkin (1991/2007) emphasized that the actual degree of difficulty was influenced by distance from the speaker, access to visual cues of the speaker, classroom noise, and the shape or configuration of the hearing loss. Typically, for a person with hearing loss, higher frequency hearing loss poses more difficulty than lower frequency hearing loss (Welling \& Ukstins, 2015). Access to high frequency consonant sounds influences one's ability to understand speech because English consonants tend to carry the meaning of speech (Welling \& Ukstins, 2015).

Negative social implications are possible with a mild hearing loss as well (Anderson \& Matkin, 1991/2007). Misunderstandings may result from missing fragments of the spoken message. Students may be accused of selective listening or daydreaming. Anderson and Matkin (1991/2007) also reported that low self-esteem may be a negative consequence; some may perceive themselves as less capable. Physical 
symptoms such as headaches and fatigue may be manifested in both children and adults with this degree of hearing loss due to the need for increased focus to hear (Welling \& Ukstins, 2015).

\section{Moderate Hearing Loss: $40-55 \mathrm{~dB}$ HL}

Both children and adults with a moderate degree of hearing loss may experience significant negative consequences (Welling \& Ukstins, 2015). As reported by Anderson and Matkin (2007), without amplification, around $80 \%$ of the speech signal may be missed with a hearing loss of $50 \mathrm{~dB}$ HL. Welling and Ukstins (2015) stated "even with appropriate amplification, a child is at a considerable disadvantage in most listening environments, especially the average noisy classroom" (p. 102). Lack of visibility of the speaker and increased distance between the speaker and listener may further impede communication (Welling \& Ukstins, 2015).

Early intervention and early amplification are emphasized for supporting the development of speech development, spoken language, and learning (Anderson \& Matkin, 1991/2007). In addition, Anderson \& Matkin (1991/2007) reported that with hearing aids alone, children are at risk for effective learning and comprehension of speech. FM systems are typically needed. In the absence of appropriate intervention, significant language delays and articulation errors will likely develop in children (Anderson \& Matkin, 1991/2007). Anderson and Matkin (1991/2007) indicated that the addition of a visual system may support communication for students experiencing delays in language or with additional disabilities.

Socialization with peers may also be compromised and self-esteem may be impacted (Anderson \& Matkin, 1991/2007). They may be accused of not attending or 
choosing to hear when they want to hear. Noisy settings, such as the school cafeteria and learning groups, may make interactions with peers more challenging. Fatigue may be manifested in this population with an increased effort needed for listening.

\section{Moderately Severe Hearing Loss: 55 - 70 dB HL}

Without amplification, individuals with a moderately severe hearing loss may miss out on everything said in typical conversations (Anderson \& Matkin, 1991/2007). Without early identification of hearing loss and early intervention, delays in speech and language development would most likely be negatively impacted. The impact on language may be far-reaching including disorders in the areas of (a) "syntax," (b) “morphology," (c) “vocabulary," and (d) "semantics" (Welling \& Ukstins, 2015, p. 103).

For children with a moderately severe hearing loss, pragmatics, academics, literacy, and social development may also be negatively affected (Tye-Murray, 2009). Especially in the presence of noise, interactions with peers and others may be compromised in group situations as well as in one-on-one situations (Anderson \& Matkin, 1991/2007). Use of an FM system can improve access to the teacher's spoken message. Use of sign language or another visual system may support learning. Given a moderately-severe hearing loss, Anderson and Matkin (1991/2007) also indicated that social maturity and self-concept may be negatively affected.

\section{Severe Hearing Loss: 70 - $90 \mathrm{~dB}$ HL}

For individuals with a severe hearing loss, there is no access to the speech signal without the use of hearing technology (Welling \& Ukstins, 2015). Cochlear implants may be an option (Cole \& Flexer, 2011). Cochlear implants are medical devices. Cochlear implants provide auditory access for individuals with bilateral, severe to 
profound hearing loss who gain insufficient benefit from amplification with hearing aids (Alexiades et al., 2008). Rather than amplifying sounds as hearing aids do (CDC Treatment and Intervention Services, 2012), cochlear implants bypass damaged inner ear hair cells (Cole \& Flexer, 2011) sending electrical sound sensations to the auditory nerve (Alexiades et al., 2008). The U.S. Food and Drug Administration approved cochlear implants for children with bilateral, profound hearing loss as young as 12 months of age (Alexiades et al., 2008; Kirk, Miyamoto, Ying, Perdew, \& Zuganelis, 2002).

Early amplification and early intervention are imperative for the development of spoken language (Cole \& Flexer, 2011). Anderson and Matkin (1991/2007) indicated that consistency in amplification coupled with intensive signed or spoken language intervention could increase the chance of developing speech and language near a typical rate. They also indicated that socialization with peers who are hearing may be challenging.

\section{Profound Hearing Loss: 90 - dB HL or Greater}

A strong connection exits between the severity of hearing loss and educational impact. Research has demonstrated that the greater the hearing loss, the greater potential for learning difficulties (Schow \& Nerbonne, 2002). Even with hearing aids, individuals with a profound hearing loss will not have access to many of the sounds of speech (Anderson \& Matkin, 1991/2007). Anderson and Matkin (1991/2007) stressed intensive intervention focused on language learning, whether in sign or spoken, for children with a profound hearing loss.

Cochlear implants are an option for many children with profound hearing loss. Cole and Flexer (2011) stressed that cochlear implant technology and focused auditory 
intervention can provide "auditory brain access and auditory neural development" (p. 43) to support the development of spoken language. With appropriate and intensive early amplification and intervention, young children with profound hearing loss who use cochlear implant technology may present more like an individual who is hard of hearing rather than profoundly deaf (Paul \& Whitelaw, 2011).

This section highlighted the categories of degrees of hearing loss and possible ramifications due to hearing loss. For children, hearing acuity levels range from normal hearing sensitivity $(0-15 \mathrm{db} \mathrm{HL})$ through profound hearing loss which falls at $90 \mathrm{~dB} \mathrm{HL}$ or greater (Cole \& Flexer, 2011). The more significant the hearing loss, the more critical role early identification of hearing loss, early amplification, and early intervention are for the child's development of speech and spoken language (Welling \& Ukstins, 2015). However, even a slight or minimal hearing loss may impede a child's development (Flexer, 1994) and affect academic achievement (Spencer \& Marshark, 2010).

\section{Educating Students who are d/Deaf or Hard of Hearing}

"Communication is at the core of our existence... [and] at the heart of expressing oneself lies language - the basic tool that in turn links us to our culture, home, community, and surrounding environment" (Scheetz, 2001, p. 107). For those with hearing, spoken language is typically learned without effort (Ling, 1989). For children with hearing loss, the development of language has been one of the most significant areas affected (Lederberg, Schick, \& Spencer, 2012). In the U.S., deaf education has been known for its controversies, with the oral-manual communication debate among one of its most volatile and long-lasting debates (Marschark et al., 2002; Moores, 2001). 
In the U.S., deaf education has realized mixed success, abundant challenges, and reoccurring failures (Moores, 2001). Even without the presence of an additional condition or disability, hearing loss during childhood places children at "high risk for language, social, and academic difficulties" (Spencer \& Marshark, 2010, p. 16). Delays in speech may also occur in students who are d/DHH (Tye-Murray, 2009). Poor literacy outcomes have been a long-standing challenge in deaf education (Luckner, 2013; Traxler, 2000). Print literacy may indeed be the most troubling challenge to date (Spencer \& Marschark, 2010). As stated in The National Agenda (2005) focused on achieving educational equality for students who are d/DHH, ...perhaps unlike any other children in this nation, [children who are $\mathrm{d} / \mathrm{DHH}$ ] continue to struggle academically, as reflected in 3rd grade reading scores, low high school and college graduation rates, alarmingly high rates of un- and -under employment, reliance on governmental assistance, and earning capacities that are $40-60 \%$ below those of their hearing counterparts. (p. 8)

However, the past two decades have also seen marked developments which resulted in significant changes in the field of deaf education (Johnson, 2013; Lenihan, 2010; Marvelli, 2010). Universal Newborn Hearing Screening (UNHS) has opened the door for earlier amplification and service provisions for infants and toddlers who are d/DHH and their families. In 1990, Hawaii became the first state in the U.S. to mandate hearing screening of all newborns (White, 2008).

Prior to UNHS, diagnosis of hearing loss occurred, on average, at about $21 / 2$ years of age (National Center for Hearing Assessment and Management, 2011). Approximately 20 years later, improved results in the early identification of hearing loss 
were remarkable (Centers for Disease Control and Prevention [CDC], 2011). According to $\mathrm{CDC}$ (2011), approximately $70 \%$ of the infants diagnosed with hearing loss were diagnosed before three months of age. Yet the screening and diagnosis of hearing loss is relatively meaningless without follow up with appropriate early intervention services (Joint Committee on Infant Hearing [JCIH], 2013).

In 2011, CDC (2011) statistics showed that nearly 98\% of infants born in U.S. states and territories were screened for hearing loss. Of those infants failing the screening, $8.6 \%(5,088)$ were diagnosed with hearing loss indicating a prevalence of hearing loss of 1.5 per 1000 screened. Of those diagnosed, $62.9 \%(3,253)$ reportedly received early intervention services. Although a number of infants were lost to follow up at the diagnostic and early intervention phases, $35.3 \%$ and $26 \%$ respectively, UNHS resulted in earlier diagnosis and intervention for many babies who were $\mathrm{d} / \mathrm{DHH}$. In addition, advances in cochlear implant technology resulted in FDA approval for cochlear implant technology for babies as young as 12 months of age (Alexiades et al., 2008; Kirk et al., 2002).

Early intervention and early use of hearing technologies appear to be changing the landscape (Paul \& Whitelaw, 2011). Paul and Whitelaw (2011) contended "with early intervention and early amplification, many individuals with severe-to-profound hearing losses can perform like hard of hearing individuals, which essentially means that they are connected to the world of sound" (p. 5). Cole and Flexer (2011) concurred.

Cole and Flexer (2011) emphasized that the degree of hearing loss has become a non-determinant in "whether a child would likely be auditory or visual in orientation" (p. 38). A child with hearing loss "can function like a child with a mild to moderate hearing 
loss... if we access the brain with appropriate technology early on (hearing aids and cochlear implants) and provide enriched auditory language stimulation” (p. 38). Yet, even a slight to mild hearing loss may affect the child's development (Flexer, 1994) and academic achievement (Spencer \& Marshark, 2010) of children. Furthermore, research has that shown children with unilateral hearing loss, a group frequently overlooked for intervention (Most \& Tsach, 2010), are at high risk for educational difficulties (Bess \& Tharpe, 1984; Northern \& Downs, 1991).

This section of the literature review addressed four key areas. First, the demographics of students who are $\mathrm{d} / \mathrm{DHH}$ was presented. Next, three primary areas regarding the outcomes of students who are $\mathrm{d} /$ Deaf and hard of hearing were addressed: (a) language development with early intervention, (b) language development with cochlear implants, and (c) reading and print literacy. The rational for selection of these student outcome areas was further explained below.

Language is "the basis of all learning" (Wang et al., 2014, p. 36). Language was addressed within the areas of early intervention and cochlear implants because of their impact on positive language outcomes within the field of educating learners who are d/DHH (Geers \& Nicholas, 2013; Nicholas \& Geers, 2008; Lenihan, 2010; MeinzenDerr, Wiley, \& Choo, 2011; Yoshinaga-Itano, 2003). Reading and print literacy was the final section highlighted due to the long-standing challenge in reading outcomes of students who are d/Deaf and hard of hearing (Luckner, 2013; Spencer \& Marshark, 2010; Traxler, 2000; Trezek et al., 2010) as well as the importance of reading living in a global society (Luckner, Sebald, Cooney, Young III, \& Muir, 2005/2006). 


\section{Student Demographics}

Individuals who are d/DHH form a heterogeneous group (Tye-Murray, 2009). There are multiple factors or characteristics that lead to diversity among its members (Marschark et al, 2002). Factors such as (a) degree of hearing loss, (b) age of onset of hearing loss, and (c) etiologies of hearing loss are just a few variables that create this diversity (Marschark et al., 2002). Modes of communication (Holden-Pitt \& Diaz, 1998) and use of different types of hearing and visual technologies and services further distinguish members from each other (Gallaudet Research Institute, 2011). Use of cochlear implant technology is on the rise (Mitchell \& Karchmer, 2006) and this trend is expected to continue.

Gallaudet Research Institute (GRI) data from the Annual Survey of Deaf and Hard-of-Hearing Children and Youth as reported by Holden-Pitt and Diaz (1998) showed $44 \%$ of the students who were $\mathrm{d} / \mathrm{DHH}$ that responded to the $1996-97$ survey used an auditory-oral method of communication. Use of sign and voice as students' main mode of communication topped that percentage at $51 \%$. Of the remaining five percent, $4 \%$ of the students who were $\mathrm{d} / \mathrm{DHH}$ reported they used sign only and $1 \%$ indicated Cued Speech.

About a dozen years later, GRI 2009-2010 Annual Survey of Deaf and Hard-ofHearing Children and Youth data showed different results (GRI, 2011). Fifty-three percent of the students responding to the survey reported using only spoken language as their primary mode of communication. Thirty-nine percent used sign language, with $27 \%$ using sign language exclusively and $12 \%$ using it in combination with speech. The remaining $7 \%$ fell into spoken language with the addition of cues or other. As reported by 
Spencer and Marshark (2010) and Mitchell and Karchmer (2006), GRI data consists of data from about $60-70 \%$ of students who are d/DHH in U.S. schools. Students who are $\mathrm{d} / \mathrm{DHH}$ and attend public schools may be underrepresented in GRI data (Mitchell \& Karchmer, 2006; Spencer \& Marshark, 2010).

The racial and ethnic demographics of the population of students who are d/DHH has also changed (Holden-Pitt \& Diaz, 1998; Johnson, 2004). Holden-Pitt and Diaz (1998) utilized data from the Annual Survey of Deaf and Hard-of-Hearing Children \& Youth collected through Gallaudet Research Institute. Holden-Pitt and Diaz (1998) identified a shift in demographics among students who were d/DHH between 1977 and 1997. Racial and ethnic backgrounds showed a decrease in white students from $71 \%$ to $58 \%$, an increase of $9 \%$ in Hispanic students between those years, resulting in $18 \%$ of those surveyed, and a 3\% increase (to 4\%) in Asian students who are d/DHH. The number of African Americans remained relatively stable, hovering at about 17\% and the population of American Indian rose slightly to $1 \%$.

Mitchell and Karchmer (2006) suggested that the national Child Count data collected was a better estimate for determining students who are d/DHH in U.S. public schools. Child Count began being collected in 1975 as mandated by the Education for All Handicapped Children's Act (Mitchell \& Karchmer, 2006). However, Holden-Pitt and Diaz (1998) showed that the proportion of changes in racial and ethnic demographics closely mirrored changes noted in the general U.S. population. Likewise, the number of White students who are $\mathrm{d} / \mathrm{DHH}$ was expected to continue to decline.

Further disparity was noted within the population of students who are d/DHH. As Spencer and Marschark (2010) reported, at least one third and perhaps as high as half of 
the population of students who are d/DHH have an additional condition or disability beyond hearing loss. Because an individual has a hearing loss, it does not preclude them from having another condition or disability as would occur in the population of students with hearing (Spencer \& Marschark, 2010).

At times, hearing loss and the presence of an additional disability occur concomitantly (Bruce, DiNatale, \& Ford, 2008). And although additional disabilities occurs less frequently in the population of individuals with hereditary deafness (Bruce et al., 2008), approximately $30 \%$ of individuals with a hereditary form of hearing loss present with a genetic syndrome which often includes additional conditions (Arnos \& Pandya, 2007). Adding further complexity to this population is that students who are d/DHH with additional disabilities do not necessarily present with the same additional disabilities or conditions (GRI, 2011).

In the Annual Survey of Deaf and Hard of Hearing Children and Youth (GRI, 2011), nearly 38,000 students who were d/DHH reported on a survey item related to the presence of additional conditions. Nearly 6,000 students did not respond to this item. Thirty-nine percent of the students indicated that they had at least one additional condition. Learning disabilities, mental retardation, and other conditions were the most frequently reported responses (GRI, 2011). Additionally, low vision, legal blindness, developmental delay, orthopedic impairment, Attention Deficit Disorder or Attention Deficit Hyperactivity Disorder, Traumatic brain injury, emotional disturbance, autism, Usher syndrome, and other health impairments were reported by students in GRI's (2011) survey. 
The varieties of existing additional disabilities among students who are d/DHH, increases the diversity among this already highly diverse population. The specific impact on learning "vary [ies] according to the severity of the additional disability" (Bruce et al., 2008, p. 370). Furthermore, "children who are deaf and hard of hearing with multiple disabilities are a heterogeneous and poorly defined subgroup" (Jones \& Jones, 2003, p. 300). And although research is lacking on this subgroup (Jones \& Jones, 2003), Spencer and Marschark (2010) reported "generally [researchers have] concluded that the combined effects of multiple disabilities are multiplicative and not merely additive" (p. $15)$.

It is also evident that the school environment in which students who are $\mathrm{d} / \mathrm{DHH}$ are being educated has changed as well (Holden-Pitt \& Diaz, 1998). During the 20092010 school year, approximately $86 \%$ of students who were $\mathrm{d} / \mathrm{DHH}$ between the ages of 3 and 21 years were enrolled in public schools in the U.S. A majority of those students, over $54 \%$, were educated in the general education classroom at a minimum of $80 \%$ of their school day (U.S. Department of Education, National Center for Education Statistics, 2012).

In the past, when a majority of placements were in residential schools or selfcontained classrooms (Moores, 2001), there was a natural occurrence of a "critical mass" (Johnson, 2004, p. 76) of students who were d/DHH. This decreased the isolation of students and increased opportunities for interpersonal and informational communications (Johnson, 2004). However, the majority of students who are d/DHH are now more spread out within their neighborhood schools (Mitchell \& Karchmer, 2006). Based on estimated of GRI Annual Survey data, Mitchell and Karchmer (2006) indicated that of 
the public schools that have students who are $\mathrm{d} / \mathrm{DHH}$, over half $(53 \%)$ of those schools have only one student who is $\mathrm{d} / \mathrm{DHH}$ receiving special education services in their school. Students who are d/DHH form an eclectic group (Tye-Murray, 2009). Age of onset of the hearing loss, cause of the hearing loss, severity of hearing loss (Moores, 2001), and mode of communication (Holden-Pitt \& Diaz, 1998) distinguishes members from each other (Moores, 2001). Furthermore, the presence of an additional disability further differentiates members of this group from each other (GRI, 2011; Mitchell \& Karchmer, 2006; Spencer \& Marschark, 2010). Diversity also exists in the type and severity of the additional disabilities (Bruce, et al., 2008; Spencer \& Marschark, 2010). In addition, this population is becoming more racially and ethnically diverse (Holden-Pitt \& Diaz, 1998; Johnson, 2004). There is an increasing number of students with cochlear implants in the educational system (Mitchell \& Karchmer, 2006) as well as an increase in those who communicate through spoken language alone (GRI, 2011). Lastly, where these students are being educated has dramatically changed with the majority learning alongside their peers who are hearing in the regular classroom (Mitchell \& Karchmer, 2006; U.S. Department of Education, National Center for Education Statistics, 2012).

\section{Language and Early Intervention}

Regardless of the mode of communication chosen for their child, it is imperative that early intervention directly follow the diagnosis of hearing loss in young children (JCIH, 2013; Wang \& Engler, 2011; Yoshinaga-Itano, 2003). Early intervention has been found to play an important role in early language development and positive outcomes have been documented (JCIH, 2013; Meinzen-Derr et al., 2011; Spencer \& Marschark, 2010; Yoshinaga-Itano, 2003). Strong parental involvement is another 
positive factor influencing the development of language in their young children who are d/DHH (Wang \& Engler, 2011; Yoshinaga-Itano, 2003).

The state of Colorado has aggressively pursued early identification and early intervention for infants and toddlers with hearing loss. To that end, the time span between identification and intervention in Colorado is only two months for most children (Yoshinaga-Itano, 2003). As reported in Yoshinaga-Itano (2003), during the nine year period between 1986 and 1995, Colorado's average age of identification of hearing loss decreased by 20 months to an impressive 3 months of age. Colorado has also been a leader in documenting early intervention results.

In a review of Colorado's early intervention studies, Yoshinaga-Itano (2003) identified an "early-identification language advantage" (p. 256) Infants who were d/DHH who were identified and received early intervention services by six months of age had significantly higher language quotients over peers with hearing loss identified after that six-month marker. This early identification/intervention effect was noted across multiple demographic variables regardless of (a) level of hearing loss, (b) ethnicity, (c) gender, and (d) communication modality of auditory-oral, sign language, or sign and spoken language.

The aforementioned advantage was found across all years tested, from birth through age five. In addition, it was present for children with and without additional disabilities as well as children with lower cognitive functioning. Yoshinaga-Itano (2003) purported that the states of Colorado, Nebraska, and Washington have demonstrated that across all three states, "early-identified/intervened children achieved similar developmental outcomes... indicating that optimal developmental outcomes for children 
with congenital hearing loss is an attainable goal for the majority...of these children" (p. 266).

Similarly, positive outcomes were also found among early identified children, those identified by six months of age, in Ohio (Meinzen-Derr et al., 2011). On average, when excluding those with medically fragile conditions, the children who were $\mathrm{d} / \mathrm{DHH}$ in Ohio's study had expressive language skills appropriate for their age. They were also shown to maintain that level during their early intervention years.

Unlike the Yoshinaga-Itano (2003) study, there was no data available after the Ohio children exited early intervention (Meinzen-Derr et al., 2011). An important finding by Meinzen-Derr et al. (2011) was that late identified children showed greater gains to their language quotients overtime than those in the early identified category, thus indicating a potential for closing the gap. Results from the aforementioned studies yielded positive outcomes and promising results. However, aside from some overlap, early intervention for young children who are d/DHH has not been shown to "match [the] typical [language] performance of hearing children" (Spencer \& Marschark, 2010, p. 47).

\section{Language and Cochlear Implants}

In Evidence-based Practice in Educating Deaf and Hard-of-Hearing Students, Spencer and Marschark (2010) reported on research on language development among children who were d/DHH using different communication modalities. Although some age-appropriate language skills were found across all methodologies, this success was not commonplace. "Regardless of modality, language delays result from lack of complete access to a language model" (Spencer \& Marschark, 2010, p. 78). Spencer and Marschark further reported that language delays frequently existed in the areas of 
vocabulary acquisition, sentence structure, and English morphological markers. Again, this was evident across all methodologies. However, Spencer and Marschark also reported on a new wave of studies. These studies, focused on cochlear implants and early intervention, have shown great strides in the development of spoken language.

Unlike Yoshinaga-Itano (2003) and Meinzen-Derr et al. (2011) studies demonstrating great strides in the development of spoken language among cochlear implant users who received early intervention services, often included children with normal intelligence and excluded children with additional disabilities (Spencer \& Marschark, 2010). Spencer and Marschark (2010) also cautioned that comparisons across studies and methodologies may be flawed. Some researchers "have limited their participants to children who are most likely to succeed...children...[with] no [additional] disabilities... and [children] whose families are intensely involved in the program" (p. 80). A study by Nicholas and Geers (2008) may demonstrate this potential flaw.

Nicholas and Geers (2008) studied 76 congenitally deaf children from 23 states and one Canadian province. Seventy-five percent of the children were White. All children included in the study had one cochlear implant with full insertion of the cochlear implant electrode array and no problems with the cochlear implant that resulted in lack of use for over 30 days. In addition, all children in the study participated in oral based programs at least since implantation with $80 \%$ of those studied attending private oral schools or enrolled in auditory-verbal therapy. Only $8 \%$ of the children received their intervention within the public schools. All families used spoken English as the primary language in the home. And only children who scored average or above average for intelligence and had no additional disabilities were included in the study. Lastly, the 
majority of participants were from households of middle to high income with $71 \%$ of the mothers having at least a four-year college degree.

Positive spoken language outcomes of children with cochlear implants who communicate through spoken language have been well documented (Geers \& Nicholas, 2013; Lenihan, 2010; Nicholas \& Geers, 2008; Spencer \& Marschark, 2010). For example, Geers and Nicholas (2013) demonstrated advantages in spoken language outcomes, "for both lexical skills and for overall receptive and expressive language" (p. 652) among children receiving cochlear implants at an early age. This advantage was continued into the middle elementary school years. In contrast, historically, children who were deaf would lose ground in language with advancing age (Geers \& Nicholas, 2013), thereby, creating a greater gap between their language performance when compared to their peers with typical hearing.

Geers and Nicholas (2013) recognized that the aforementioned study included "a relatively advantaged group of CI [cochlear implant] recipients" (p. 646). Again, their sample of participants excluded children with additional disabilities. In addition, family incomes were "well above average, and parents were motivated to help their own child... as evidence by their willingness to accompany their child to a research camp some distance from their home" (p. 647). Regardless of the parameters, the spoken language outcomes for children with cochlear implants who use spoken language are encouraging and positive. Indeed, early identification of hearing loss followed by intensive auditory-oral intervention has resulted in improved spoken language outcomes for some young children who are deaf (Geers \& Nicholas, 2013; Spencer \& Marschark, 2010). 
Cochlear implants have also yielded some positive results within other groups of individuals who are d/DHH (Beer, Harris, Kronenberger, Holt, \& Pisoni, 2012; Kirk et al., 2002). Kirk et al. (2002) study of 106 cochlear implant users who were prelingually deaf, showed improvement in communication skills overtime, yet, on average, their language age fell below same age peers with hearing. Children who received their cochlear implant before the age of two had better language outcomes.

Kirk et al. (2002) found "no significant differences in rates of language development between the oral and Total Communication groups" (p. 127). However, "spoken word development was significantly greater for children who used oral communication, than for children who used Total Communication" (p. 127). And overall word recognition was better for the group who received their cochlear implant at or after the age of five. Familiarity with vocabulary may have been a factor.

Lastly, Beer et al. (2012) studied 23 children with cochlear implants who had an additional disability. The additional disability present was different among participants. They matched these children with 23 children who also had cochlear implants, but no additional disability. Language quotient results indicated that although the group of students with a cochlear implant and an additional disability made progress, they lagged behind their matched peers.

Marvelli (2010) stated "cochlear implants have provided... [oral deaf] children with the potential for living and learning alongside their peers with typical hearing" (p. 110). He further indicated that the focus of educating students who are $\mathrm{d} / \mathrm{DHH}$ has shifted to the birth to 5 population, "without forgetting that these children also may need special services throughout their years of schooling" (p. 110). Yet, although gains in the 
development of spoken language for the cochlear implant recipients have been significant, language scores have not yet yielded commensurate outcomes when compared with peers with typical hearing (Geers \& Nicholas, 2013; Spencer \& Marschark, 2010).

\section{Reading and Print Literacy}

As previously stated, deaf education has yielded dismal results in the area of reading for students who are d/DHH (King \& Quigley, 1985; The National Agenda, 2005; Traxler, 2000; Trezek, Wang et al., 2010). And these poor literacy outcomes have been long-standing (Luckner, 2013; Traxler, 2000). The reading levels of most deaf students with severe-to-profound hearing loss lagged behind their peers with hearing upon high school graduation (Traxler, 2000).

More alarming is that for years, standardized achievement tests showed a fairly stable plateau for many readers, with severe-to-profound hearing loss placing them near a third or fourth grade reading level (King \& Quigley, 1985; The National Agenda, 2005; Traxler, 2000). Although successful readers exist (e.g., Luckner \& Muir, 2001; Wang, Spychala, Harris, \& Oetting, 2013), unfortunately, "for the majority of students who are deaf or hard of hearing, learning to read... is a tortuously slow and frustrating process" (Luckner et al., 2005/2006, p. 444).

There is agreement among researchers in the field that the low reading levels among students who are d/DHH is unacceptable (Allen et al., 2009; The National Agenda, 2005; Trezek et al., 2010). Where agreement among researchers is lacking is on the best approach for teaching reading to students who are d/DHH (Allen et al., 2009; Paul, Wang, Trezek, \& Luckner, 2009; Syverud, Guardinao, \& Selznick, 2009; Wang, 
Trezek, Luckner, \& Paul, 2008). Also, some professionals cling to a deeply held belief regarding a particular mode of communication being more suited for the development of reading skills with the mode of choice differing among professionals (Trezek et al., 2010).

Irrespective of modes of communication used to establish language of children who are $\mathrm{d} / \mathrm{DHH}$, a body of evidence is beginning to emerge in support of direct instruction for literacy skill development (Spencer \& Marschark, 2010). Direct instruction of literacy skills may warrant changing teacher preparation programs to accommodate improved instructional strategies taught to teacher candidates (Trezek et al., 2010), with the hopeful outcome that these strategies are implemented with students who are d/DHH in schools. Specifically, a meaningful focus on direct teaching of (a) vocabulary, (b) syntax, and (c) phonology through interactive contexts is deemed necessary (Spencer \& Marschark, 2010).

"Phonological awareness and print knowledge are strongly predictive of later reading abilities" (Ambrose, Fey, \& Eisenberg, 2012, p. 817). Research suggests that students, including those who are d/DHH, "need to understand the connection between the phonemes of a phonetic language and the graphemes of print, especially for a language such as English" (Trezek et al., 2010, p. 17). Trezek et al. (2010) and Wang et al. (2008) contended that students who are d/DHH may access phonemic awareness and phonics skills through a visual modality; audition alone may not be sufficient for this group. Wang et al. (2008) purported that Visual Phonics or Cued Speech in combination with speechreading should be incorporated through direct instruction to teach these phonological skills. Not all researchers agreed (Allen et al., 2009). 
In a critical response to Wang et al. (2008), Allen et al. (2009) purported "only a weak correlation [existed] between phonological coding and reading in deaf readers" ( $\mathrm{p}$. 339). Allen et al. further argued that Wang et al. (2008) inaccurately represented Cued Speech, missed the mark by not including a discussion regarding "the role of sign language in the acquisition of reading" (p. 340), among other claims. Paul, Wang et al. (2009) countered with a rejoinder in which they continued to emphasize the importance of phonology in learning to read, also recognizing that it isn't the only factor of relevance.

Paul et al. (2009) stressed that not only do all children, those with hearing included, need to develop English phonology; but, that it is accessible to all students regardless of mode of communication and level of hearing acuity. Perhaps the best response for Wang et al. (2008) would be sharing results of research conducted at a clinic preschool for young children who were d/DHH within the Midwest. Two years postintervention, Wang et al. (2013) showed age and above age-appropriate reading levels found in three highly diverse students across three different levels of hearing loss, three different modes of communication, and three different types of amplification, including no amplification at all for one of the students.

As previously stated, studies are beginning to show support for direct instruction for literacy skill development (Spencer \& Marschark). Wang et al. (2008) and others (Ambrose et al., 2012; Syverud et al., 2009; Wang et al. 2013) explored phonological skill development in children who are d/DHH. Syverud et al. (2009) conducted a case study of a 7 year old first grader with a progressive, bilateral, moderate-to-severe hearing loss who was "struggling with reading" (p. 384). Given eight weeks of intervention using 
a 1983 curriculum of direct instruction, Teach Your Child to Read in 100 Easy Lessons

by Engelmann, Haddox and Brunner, the student demonstrated progress in the phonological skill of decoding.

Ambrose et al. (2012) set out to determine if 24 preschoolers with cochlear implants, those presenting with no cognitive concerns and with no additional disabilities, demonstrated age-appropriate skills in phonological awareness and print knowledge as compared with peers who were hearing. All of the children with cochlear implants in the study had their cochlear implant for at least 18 months. And although, mode of communication was not specified for the group of cochlear implant users, only spoken language users participated in the study.

Both the children with cochlear implants and the children with typical hearing were between the ages of 36 and 60 months and both were from English speaking home environments (Ambrose et al., 2012). Likewise, the children with hearing had no additional disabilities. The average score on the phonological awareness measure for the cochlear implant users was "slightly more than one standard deviation below the mean score [of matched hearing peers]... and this difference was statistically significant” (p. 816). Print knowledge scores yielded no significant difference between the two groups. As previously mentioned, Wang et al. (2008) indicated that Visual Phonics or Cued Speech could be used to support the development of phonology, specifically phonemic awareness and phonics skills. Visual Phonics, formally titled See-theSound/Visual Phonics, is a multi-sensory approach to assist in the "development of an inner phonological coding related to English structure" (Waddy-Smith \& Wilson, 2005, p. 6). The 46 hand cues have corresponding symbols to make the grapheme-phoneme 
connection (Trezek et al., 2010). Individuals who use sign language or spoken language may utilize Visual Phonics (Waddy-Smith \& Wilson, 2005).

Cued Speech is a system for transmitting language (Smith, 2007). Cues are rendered meaningless without being paired with speechreading. There are eight handshapes that are positioned in four different locations around the mouth. When the cues are coupled with speechreading it completes the spoken language picture, thus providing information that differentiates the phonemes that look the same on the lips. Cued Speech may be used by individuals across a variety of communication modalities (Smith, 2007).

Wang et al (2013) explored the impact of an early intervention phonics-based intervention which incorporated Visual Phonics, on the reading skills and grade level reading outcomes of three, young, diverse students who were $\mathrm{d} /$ Deaf of hard of hearing. All three students attended a clinic-based, language-intensive preschool program for students who are d/DHH. Each preschooler received 40-50 weeks of phonics-based intervention. And each preschooler differed in (a) degree of hearing loss, (b) modes of communication, and (c) use of hearing technologies, including no amplification.

Given training, all three preschoolers demonstrated "some use of phonemic awareness and phonics skills, and... these skills were maintained in early elementary school" (Wang et al., 2013, p. 107). At two years post-intervention, all three students who attended elementary schools in three different cities displayed an overall functioning at or above grade level for reading. In light of the often homogenous and "relatively advantaged" (Geers \& Nicholas, 2013, p. 646) cochlear implant groups in studies 
regarding spoken language and reading outcomes, Wang et al. (2013) study of an eclectic group of students who are d/DHH may yield further merit.

Specifically, the first student in the Wang et al. (2013) study used primarily oral communication. This student had a moderate hearing loss that presented bilaterally. She wore two hearing aids and utilized an FM system. Student two was diagnosed with a central hearing loss as well as a language processing disorder. This student used no amplification. Her only mode of communication was ASL. Preschool provided her first experience in an intensive, language-rich environment. The third and final student in the study had a profound, bilateral hearing loss. This student had a cochlear implant and wore a hearing aid on her contralateral ear. She communicated simultaneously through use of spoken English and sign language.

Each of the aforementioned students attended the same preschool and received phonics-based group instruction and individualized instruction (Wang et al., 2013). Upon graduation from preschool, the students attended three different public schools in three different cities. When tested in early elementary school, two of the students, the first and second described above were in first grade. The third student, the cochlear implant user was in kindergarten. All students "demonstrated at least some use of phonemic awareness and phonics skills... [and] these skills were sustained in early elementary school" (Wang et al., 2013, p. 107). Furthermore, as previously mentioned all three students tested at or above age-appropriate level in the area of reading when tested in early elementary school as compared to peers with typical hearing.

Outcomes for students who are d/DHH have been mixed. Although there are success stories across all communication methodologies, too many students have fallen 
short of reaching their full potential. Students who are d/DHH are at risk for academic, language, and social difficulties (Moores, 2001). Reading and print literacy have yielded dismal results for student who are d/DHH (Luckner, 2013; Spencer \& Marschark, 2010; The National Agenda, 2005; Traxler, 2000). However, significant positive outcomes in both language and literacy have emerged.

Early identification of hearing loss, improved access to auditory information through cochlear implant technology, and children receiving early intervention at earlier ages has resulted in great strides in the field (e.g., Geers \& Nicholas, 2013; Meinzen-Derr et al., 2011; Nicholas \& Geers, 2008; Yoshinaga-Itano, 2003). This group, however, continues to lag behind same age peers with hearing (Spencer \& Marschark, 2010). There is also an emerging body of evidence that supports direct instruction for literacy skill development (e.g., Trezek et al., 2010; Wang et al., 2013). Positive literacy outcomes have resulted (Trezek et al., 2010; Wang et al., 2013).

\section{Educating Deaf Education Teacher Candidates}

There is a critical need for teachers of students who are d/DHH in U.S. public schools (American Association for Employment in Education, 2008; U.S. Department of Education, 2012); yet, U.S. deaf education teacher preparation programs have continued to decline (Johnson, 2013). More are at risk of closing their doors (Benedict et al., 2011). It is imperative that deaf education teacher preparation programs that remain, prepare teacher candidates to meet the heterogeneous population of students who are $\mathrm{d} / \mathrm{DHH}$ they serve.

There are notable trends in deaf education as well as a variety of challenges facing deaf education teacher preparation programs as they prepare teacher candidates to teach 
students who are d/DHH (Andrews \& Covell, 2006/2007; Ausbrooks, Baker, \& Daugaard, 2012; Benedict et al., 2011; Dolman, 2010; Lenihan, 2010; Johnson, 2004; Johnson, 2013; Spencer \& Marshark, 2010). This section of the paper (a) identified the population of educators of students who are $\mathrm{d} / \mathrm{DHH}$, (b) reviewed the growing knowledge base and skill set needed for educators of students who are d/Deaf and hard of hearing, and (c) explored the functioning of deaf education teacher preparation programs in the current literature. In closing, the need for this study was solidified.

\section{Educator Demographics}

It has been well documented that students who are $\mathrm{d} / \mathrm{DHH}$ are a heterogeneous group (Andrews \& Covell, 2006/2007; Gallaudet Research Institute, 2011; Moores, 2001, Spencer \& Marshark, 2010; Tye-Murray, 2009). In contrast, teachers of student who are d/DHH are a highly homogeneous group (Simms, Rusher, Andrews, \& Coryell, 2008). Rice and Goessling (2005) reported that special education field was dominated by females. Moores (2001) reported that deaf education teachers are not only typically female, but, also, typically hearing. Ausbrooks et al. (2012) and Simms et al. (2008) further stipulated that most teachers of students who are $\mathrm{d} / \mathrm{DHH}$ are White, female, and hearing.

Simms et al. (2008) conducted a study to describe the diversity of deaf education professionals, namely, teachers and administrators. Their study included collecting data from professionals in U.S. public schools, residential schools for the deaf, and deaf education teacher preparation programs. Professionals in 43 of the 50 states responded to the survey. Of the nearly 3,300 professionals surveyed by Simms et al. (2008), it is the teacher percentages taken from the data across 313 programs that are included here. 
Only $2.5 \%$ of the teachers who responded were both deaf and persons of color. When considering these areas of diversity separately, the numbers rose to nearly $22 \%$ for teachers in each category. These percentages were not significantly different from data collected approximately one decade earlier (Simms et al., 2008) nor do they mirror the race and ethnicity of the students who are d/DHH whom they serve (Gallaudet Research Institute, 2011).

According to Gallaudet Research Institute (2011), 46\% of the surveyed students who were $\mathrm{d} / \mathrm{DHH}$ self-reported as White with the remaining percentages as follows: (a) $25 \%$ Hispanic, (b) 14\% Black, (c) 3\% Asian, (d) 2\% Native Hawaiian or Pacific Islander, and (e) $6 \%$ reported to be of multi-ethnic background. Perhaps of further relevance is that the majority of teachers who were deaf, teachers of color, and male teachers were found in residential schools for the deaf (Gallaudet Research Institute, 2011), a school environment that has declined in enrollment numbers (Mitchell \& Karchmer, 2006) and the number of schools.

The trend toward racial and ethnic diversity among students who are d/DHH is projected to continue (Holden-Pitt \& Diaz, 1998; Johnson, 2004; Simms et al., 2008). Simms et al. (2008) and others (Andrews \& Covell, 2006/2007; Ausbrooks et al., 2012: LaSasso \& Wilson, 2000) contended that teacher preparation programs should prepare more diverse teachers. Andrews and Covell (2006/2007) offered recruitment strategies such as increasing university students' access to visual technologies (e.g., CART, Communication Access Realtime Translation) and recruiting at Deaf events and conferences to increase the number of deaf professionals in the field. 
Ausbrooks et al. (2012) recognized the advantage of having Deaf adult role models who are fluent in ASL for students who are deaf. They omitted any reference to oral deaf adults. What Ausbrook et al. (2012) failed to mention was the potential positive influence that oral deaf adults may have on students who are $\mathrm{d} / \mathrm{DHH}$ and use spoken language as their primary mode of communication.

Dolman (2010) completed a study on enrollment trends in deaf education teacher preparation programs with a focus on a 36 span of years from 1973-2009. He estimated ratios of deaf education teacher preparation graduates to students who are $\mathrm{d} / \mathrm{DHH}$ at 1:32 in 1973 and 1:56 in 2006. Although Dolman reported this change was relatively stable, Mitchell and Karchmer (2006) found the population of students who are d/DHH to be more widely dispersed "among a greater number of schools and programs" (p. 101). In fact, Mitchell and Karchmer (2006) reported that "more than half (53\%) of schools serving deaf and hard of hearing students have only one such student in that school" ( $p$. 99). Thus when considering the 1:56 teacher to student ratio estimated by Dolman (2010) and the greatly dispersed population of students who are $\mathrm{d} / \mathrm{DHH}$, the role of the teacher has changed. There are an increasing number of itinerant teachers of students who are d/DHH (Luckner \& Howell, 2002).

While the demographics of students who are d/DHH has evolved to include more racial and ethnic diversity, the demographics of educators of students who are $\mathrm{d} / \mathrm{DHH}$ remained relatively unchanged. The majority of educators of students who are d/DHH continues to be White, female, and hearing (Ausbrooks et al., 2012; Simms et al., 2008). The segment of the population of educators of the $\mathrm{d} /$ Deaf and hard of hearing found to be the most diverse were in residential schools for the deaf (GRI, 2011), a school 
environment that has declined in number (Luckner \& Muir, 2001). The role of the educator has needed to change to keep up with the demographic changes within this population (Luckner \& Howell, 2002).

\section{Educators' Need for Increased Knowledge, Skills, and Experiences}

Johnson (2013) and others (Dodd \& Scheetz, 2003; Luckner \& Howell, 2002;

Teller \& Harney, 2005/2006) identified “an ever-expanding array of knowledge, skills, and experiences" (Johnson, 2013, p. 441) necessary for educators to teach students who are $\mathrm{d} / \mathrm{DHH}$. Information was gathered from a variety of sources such as (a) deaf education teacher preparation faculty, (b) program directors of programs that teach students who are d/DHH and, (c) teachers of students who are d/DHH. Given the highly diverse population of students who are $\mathrm{d} / \mathrm{DHH}$, it stands to reason that the areas identified were also highly diverse.

Teller and Harney (2005/2006) surveyed program directors of students who are d/DHH. Using a computer generated random selection process, 100 programs were selected among a possible 643 programs in North America as identified through the 2004 American Annals of the Deaf reference issue. There was a 19\% return rate; therefore, 19 out of the 100 invited to participate, actually participated. Teller and Harney (2005/2006) wanted to determine directors' perceptions of competencies needed by teachers of students who are d/DHH. Although, the return rate was low, programs represented included parent-infant through high school and young adults up to 21 years and included a variety of school placements as well as modes of communication used by teachers and students. 
When program directors were asked an open ended question regarding suggestions for change in deaf education teacher preparation programs, a variety of answers resulted (Teller \& Harney, 2005/2006). The most frequently occurring responses included the need for increased experiences, skills and knowledge. Program directors believed teacher candidates should have more experiences with (a) students who were d/DHH who had additional disabilities, (b) using and maintaining hearing technologies including FM systems, (c) implementing auditory-verbal therapy, (d) working with students who use cochlear implant technology, and (e) experience in itinerant and resource placements. Additional skills were believed to be needed in sign language and addressing mental health issues. Furthermore, program directors suggested that teacher candidates needed increased knowledge of the general curriculum and meeting the No Child Left Behind Act requirements.

Dodd and Scheetz (2003) completed a statewide needs assessment in Georgia. They targeted the teachers of students who were $\mathrm{d} / \mathrm{DHH}$ to determine how prepared they were to work with students who were $\mathrm{d} / \mathrm{DHH}$. They were also interested in determining what they saw as critical to include in teacher preparation programs. One hundred and ten teachers responded to the survey from a potential pool of 250 participants. The teachers graduated from deaf education teacher preparation programs in 34 different states.

As reported by Dodd and Scheetz (2003), over half of the teachers believed they received appropriate preparation in their teacher preparation programs. The teachers also believed there were gaps in their teacher preparation programs. They stated they needed additional training in the following areas (a) ASL skills, (b) written language assessment 
for students who are $\mathrm{d} / \mathrm{DHH}$, (c) assistive listening devices, primarily cochlear implant technology and FM systems, (d) IEP development, (e) coordination of services for their students, and (f) integration of the general curriculum.

Luckner and Howell (2002) interviewed 25 itinerant teachers of students who are d/DHH. They were interested in identifying knowledge, skills, and experiential learning needed to function in an itinerant role. A consultative role was deemed most important by almost every teacher interviewed. Their job responsibility rated as providing the most impact was divided between their consultative role and their teaching role in which they provided direct instruction to their students.

The teachers who participated in the Luckner and Howell (2002) study identified key skill areas which they believed were important for inclusion in deaf education teacher preparation programs. Working well with people was emphasized. They suggested interpersonal skill development as well as consultative and collaborative skills were needed to work with professionals and with families.

Coursework was deemed important but not sufficient to develop skills needed by itinerant teachers of students who are d/DHH (Luckner \& Howell, 2002). Field experiences should also be incorporated into these areas of skill development. Luckner and Howell (2002) also found that the teachers believed an itinerant student teaching placement was important. Likewise they mentioned other areas that were also reflected by other researchers such as working with hearing technology, developing auditory skills in students, the development of self-advocacy skills in their students, and working with students with additional disabilities. And one of their most significant challenges reported impacting their effectiveness as teachers was time limitations. This was no 
surprise given that the teachers reported that driving was the second most frequent task they performed (Luckner \& Howell, 2002).

In a review of studies, Johnson (2013) summarized topic areas of knowledge and skills needed by teachers of students who are $\mathrm{d} / \mathrm{DHH}$. Some identified topic areas were specific to teachers of students who were $\mathrm{d} / \mathrm{DHH}$ and others were seemingly applicable to all teachers. Furthermore, some seemed to more closely relate to communication methodologies while others seemingly related to all students regardless of mode of communication.

According to Johnson (2013) researchers recognized the need for deaf educators to stay abreast of the advances in hearing technology and the development of listening and spoken language. Researchers also emphasized the importance of proficiency in sign language and bilingual instruction. Johnson (2013) further reported that an understanding of service delivery for individuals who are d/DHH across different environments throughout the educational spectrum was needed. This included early intervention, center-based programs, itinerant education, residential schools, and education in the general classroom, and secondary transition.

Johnson (2013) further reported that there was a recognized the need for evidence-based teaching and assessment across the curriculum for students who are $\mathrm{d} / \mathrm{DHH}$, including those with additional disabilities. In addition, uses of technologies, assistive and instructional, were included as well as the need to develop students' advocacy skills. Lastly, several items that would seemingly relate to all teachers were: (a) behavior management, (b) collaboration skills for working with parents and other professionals, (c) cultural knowledge and skills for working with multi-cultural and 
multi-lingual students, and (d) understanding and application of general education benchmarks, curriculum, and assessment mandates.

Complexities exist in deaf education (Johnson, 2013) and the role of teachers of students who are d/DHH has changed (Luckner \& Howell, 2002). Teachers of students who are d/DHH need to keep up with the changing job responsibilities (Luckner \& Howell, 2002), technologies (Dodd \& Scheetz, 2003; Johnson, 2013; Luckner \& Howell, 2002; Teller \& Harney, 2005/2006), and the diverse students they serve. A variety of professionals have recognized that the knowledge and skills needed to teach students who are d/DHH has expanded (Dodd \& Scheetz, 2003; Johnson, 2013; Luckner \& Howell, 2002; Teller \& Harney, 2005/2006). Deaf education teacher preparation programs are challenged with responding to this growing need to prepare teacher candidates for the students they will serve (Johnson, 2013; Luckner \& Howell, 2002).

\section{Deaf Education Teacher Preparation Program Functioning}

The expanding knowledge, skills, and experiences needed by teachers of students who are d/DHH has been well documented (Dodd \& Scheetz, 2003; Johnson, 2013; Luckner \& Howell, 2002; Teller \& Harney, 2005/2006). This comes at a time when deaf education teacher preparation programs are on the decline (Johnson, 2013). Benedict et al. (2011) completed a study of deaf education teacher preparation faculty. They attempted to target all deaf education teacher preparation programs in the U.S. gathered from two sources: (a) the 2007 American annals of the Deaf annual reference issue and, (b) the Michigan State University's program web-site. Prior to contacting the programs, six of the programs listed had closed bringing the total of possible deaf education teacher preparation programs to 60 . 
Out of the 60 deaf education teacher preparation programs, faculty representing 48 programs agreed to participate. Of the 48 programs, there was a response rate of $80 \%$. Of those who responded, half of the faculty in deaf education teacher preparation programs were reported to hold non tenure track, part-time positions (Benedict et al., 2011). Only a third of the faculty held full-time, tenure track positions. And seven of the institutions represented had no full-time faculty running their programs. Benedict et al. (2011) indicated the data suggested that the small body of full-time, tenure track professors were "responsible for program development, research, and leadership in deaf education" (p. 10). The researchers further reported that anecdotal evidence suggested that the full-time, tenure track faculty may take on additional teaching and administrative responsibilities to maintain program viability.

Teachers of students who are $\mathrm{d} / \mathrm{DHH}$ are needed across the U.S. (American Association for Employment in Education, 2008; U.S. Department of Education, 2012). Researchers have identified an expanding knowledge, skill set, and experiences needed by teacher candidates (Dodd \& Scheetz, 2003; Johnson, 2013; Luckner \& Howell, 2002; Teller \& Harney, 2005/2006). There is also a declining number of deaf education teacher preparation programs (Johnson, 2013), and limited full-time deaf education teacher preparation faculty to meet the demands for preparing teacher candidates (Benedict et al., 2011). It begs into question, how existing deaf education teacher preparation programs are performing.

There has been an influx of U.S. deaf education teacher preparation programs in the literature (Compton, Niemeyer, \& Michael, 2004; Hayes, 2010; Houston, 2010; Humphries \& Allen, 2008; Lenihan, 2010; McGinnis, 2010; Paterson \& Cole, 2010). Out 
of these seven programs, six of the programs are listening and spoken language focused (Compton et al., 2004; Hayes, 2010; Houston, 2010; Humphries \& Allen, 2008; Lenihan, 2010; McGinnis, 2010; Paterson \& Cole, 2010), which align closely with CED’s (2013) auditory-oral philosophy. The remaining program is an ASL/English bilingual program, aligning with CED's bilingual-bicultural approach (Humphries \& Allen, 2008). None of the programs espoused a comprehensive philosophy.

Six of the deaf education teacher preparation programs are at the graduate level (Hayes, 2010; Houston, 2010; Humphries \& Allen, 2008; Lenihan, 2010; McGinnis, 2010; Paterson \& Cole, 2010) and one is at the undergraduate level (Compton et al., 2004). One of the programs is designed as a distance education program (McGinnis, 2010); however, about half of the courses are offered online and the other half are offered in-house. Only one of the programs includes a university laboratory school (Houston, 2010). And only one of the programs addressed program effectiveness through a study (Humphries \& Allen, 2008).

Educators of students who are d/Deaf and hard of hearing are needed (American Association for Employment in Education, 2008; U.S. Department of Education, 2012); yet, deaf education teacher preparation programs are struggling to survive (Benedict et al., 2011; Johnson, 2013). Researchers identified an expanding knowledge and skill set needed for teacher candidates to face the growing and changing needs of students who are d/DHH (Dodd \& Scheetz, 2003; Johnson, 2013; Luckner \& Howell, 2002; Teller \& Harney, 2005/2006); yet, there are a limited number of full-time faculty lines in deaf education teacher preparation programs to meet this need (Benedict et al., 2011). Current 
research is lacking on the functioning of comprehensive deaf education teacher preparation programs.

\section{Summary}

The complexities of deafness are often misunderstood by the hearing majority (Moores, 2001). The dichotomy of the medical (Moores, 2001; Paul \& Jackson, 1993) and sociocultural (Moores, 2001; Schirmer, 2001) perspectives has caused tension (Paul \& Whitelaw, 2011) as has the oral-manual communication debate (Moores, 2001; Marshark et al., 2002). Regardless of one's philosophical stance, individuals who are $\mathrm{d} / \mathrm{DHH}$ loss often face a variety of challenges that not only impact academic achievement, but also social development (Anderson \& Matkin, 1991/2007; Flexer, 1994; Tye-Murray, 2009; Welling \& Ukstins, 2015). Notable and long-standing challenges in the education of students who are $\mathrm{d} / \mathrm{DHH}$ have been in the areas of language and literacy (Johnson, 2013; Luckner, 2013; Spencer \& Marshark, 2010; Traxler, 2000).

In the past couple of decades, the tide has begun to turn. Early identification of hearing loss, early intervention, and improved hearing technologies (e.g. cochlear implants) have yielded positive outcomes (e.g., Geers \& Nicholas, 2013; Lenihan, 2010; Marvelli, 2010; Nicholas \& Geers, 2008; Yoshinaga-Itano, 2003). However, research has shown that even those students who are d/DHH that are "relatively advantaged" [e.g., those student with normal or above normal intelligence and no additional disabilities] (Geers \& Nicholas, 2013, p. 646) typically lag behind in both language and literacy when compared with their peers with typical hearing (Geers \& Nicholas, 2013; Spencer \& Marschark, 2010). And while the demographics of students who are d/DHH has 
changed, the demographics of teachers who educate these students has primarily remained the same, White, hearing, and female (Simms et al., 2008).

Teachers of students who are d/DHH are in high demand within U.S. public schools (American Association for Employment in Education, 2008; U.S. Department of Education, 2012). Yet, the programs responsible for preparing these teachers, deaf education teacher preparation programs, have declined and more are at risk of closing their doors (Benedict et al., 2011). If the programs in risk of closing actually close, the Midwest will be hit the hardest (Benedict et al., 2011). There is a gap in the literature of how deaf education teacher preparation programs function, especially, those with a comprehensive philosophy. There is also a gap in the literature on programs that function while simultaneously serving students who are d/DHH with an on-site preschool program. This study explored Midwest University's deaf education teacher preparation and its people, specifically the teacher candidates and how they were empowered and enabled to learn (Marquardt, 2011). Midwest University prepared deaf education teacher candidates while simultaneously providing services to preschool children who are d/DHH and their families. 


\section{CHAPTER THREE}

\section{DESIGN AND METHODOLOGY}

In the United States, a critical need exists for public school teachers of students who are d/DHH (American Association for Employment in Education, 2008; U.S. Department of Education, 2012). Yet, the number of deaf education teacher preparation programs is on the decline (Dolman, 2008; Johnson, 2013). Thirty percent of the states within the U.S. (15 out of 50 states) are reported to have no deaf education teacher preparation programs (Deaf Education Teacher Preparation Programs, 2012).

Benedict et al.'s (2011) study of deaf education teacher preparation faculty uncovered three deaf education programs in the process of closing their doors and an additional seven programs at-risk of closing. If those programs indeed close, the Midwest will be hit the hardest. Only nine of the 15 programs in existence would remain, thus resulting in "a $40 \%$ decrease in the region's capacity to prepare teachers of deaf and hard of hearing students" (p. 7). It is imperative that states with teacher preparation education of the $\mathrm{d} /$ Deaf and hard of hearing programs work to close the gap between the shortage of deaf educators and the need for deaf educators in public school systems across the country.

Attention to the plight of deaf education has resulted in a push for change in deaf education teacher preparation programs (Johnson, 2004, 2013; Lenihan, 2010). Advances in technologies, early identification of hearing loss, and early intervention have also sparked the need for change (Lenihan, 2010; Marvelli, 2010). Researchers have identified challenges and proposed solutions (Humphries \& Allen, 2008; Johnson, 2004; Johnson, 2013; Lenihan, 2010; Paterson \& Cole, 2010); yet, little is known about the 
success of education of the $\mathrm{d} /$ Deaf and hard of hearing teacher preparation programs that exist. Even less is known about the functioning of these programs and how they prepare teacher candidates while simultaneously providing services to learners who are $\mathrm{d} / \mathrm{DHH}$. This researcher sought to describe a Midwest's deaf education teacher preparation program and its teacher candidates through a "detailed understanding of a central phenomenon" (Creswell, 2008, p. 51).

Qualitative researchers seek to understand the essence of the lived experiences and perspectives of others to understand the world (Hatch, 2002). Specifically, qualitative case studies "examine the relationship between people and structures" (SavinBaden \& Major, 2013, p. 168). As postulated by Emerson, Fretz, and Shaw (1995) a qualitative research design provided the most thick and rich data for an in-depth exploration of the phenomenon within the context of a single deaf education teacher preparation program.

This single case, Midwest University's graduate, deaf education teacher preparation program received national approval from the Council on Education of the Deaf (CED) during academic year 1988-1989 (Midwest University '88-'89 Accomplishments, 1989). For 25 years, this deaf education program has prepared teachers of students who are $\mathrm{d} / \mathrm{DHH}$ while simultaneously providing services to preschoolers who are d/DHH. Currently, the program functions with one full-time academic faculty and two full-time clinical faculty members. In addition, per course faculty are hired on a class by class basis to meet programming needs. During the threeyear timeframe of this study, five per course faculty were utilized to assist in teaching 
required deaf education teacher preparation classes. The researcher of this study is one of the two full-time clinical faculty members.

This chapter begins with a brief statement of the research purpose and research questions. Next, the researcher highlights the research design and methods providing thoughtful rationales for the proposed design and sample selection. Then, data collection gathering tools and data analysis are presented with an explanation of human subjects' protection. Next, the researcher's positionality was delineated along with strategies that were used to address study quality, specifically: (a) trustworthiness, (b) dependability, and (c) transferability. This paper concluded with a section on study limitations, assumptions, a summary, and appendices of interview, survey, and document protocols that guided this qualitative inquiry. This phenomenological case study explored Marquardt's (2011) learning organization people subsystem's “empowered and enabled to learn" (p. 26) phenomenon.

\section{Purpose}

The intent of this phenomenological case study was to provide a detailed understanding of a central phenomenon as it relates to Midwest University's comprehensive, graduate deaf education teacher preparation program and its teacher candidates. It was bounded by a single case and the number of years to be studied, three. The program's deaf education teacher candidates were the primary participants identified for this study. They were selected for study because they were most central to the circle of influence of this deaf education teacher preparation program and because they were, in part, the future teachers required to assist in filling the critical shortage of educators of students who are d/DHH in public schools across the U.S (American Association for Employment in Education, 2008; U.S. Department of Education, 2012). 
Little is known about the quality of existing deaf education teacher preparation programs. Specifically, this study explored the essence of Marquardt's (2011) learning organization people subsystem's phenomenon, empowered and enabled learning. Empowered and enabled learning was generally defined by the three overarching professional educator frames adopted and approved by Missouri's Department of Elementary and Secondary Education (MO-DESE) (Missouri Department of Elementary and Secondary Education, MO-DESE, 2011). All teaching standards and quality indicators fall within the three professional educator frames (MO-DESE, 2011). The three pillars are (a) commitment to the profession, (b) proficiency in practice, and (c) learning impact (p. 9). These three professional educator frames were used to guide the evaluations of all Missouri teacher candidates and teachers throughout the state with a goal "to ensure that students in Missouri public schools continually grow and improve" (MO-DESE, 2011, p. 3).

\section{Research Questions}

Within the context of this study, the following four research questions were addressed:

1. How does the program empower and enable teacher candidates to learn commitment to the Deaf education profession?

2. How does the program empower and enable teacher candidates to learn proficiency in practice with students who are d/Deaf or hard of hearing?

3. How does the program empower and enable teacher candidates to create learning impact in students who are $\mathrm{d} /$ Deaf or hard of hearing?

4. How could the program improve to empower and enable the learning of teacher candidates who are prepared to teach students who are d/Deaf or hard of hearing? 


\section{Design for the Study}

This qualitative research study was bounded by one case, Midwest University's comprehensive, graduate, deaf education teacher preparation program. This study investigated the program and its teacher candidates over three academic years from August 2011 through May 2014. A case study by methodology, phenomenology, was the research approach used in this study. As Savin-Baden and Major (2013) argued by blending a specific methodology with a case study it, "creates an altered and synergistic version of the approach, making the approach...more holistic, particularistic, contextual, descriptive and concrete" (p. 157). The phenomenon of study, empowered and enabled learning, was drawn from Marquardt's (2011) learning organization people subsystem.

In the field of deaf education, a field in which teacher preparation programs are struggling to survive (Benedict et al., 2011), it is critical that existing teacher preparation programs prepare teacher candidates who are able to positively impact student learning (MO-DESE, 2011). The researcher explored how one deaf education teacher preparation program prepared teacher candidates who were "empowered and enabled to learn" (Marquardt, 2011, p. 26). This qualitative research focused specifically on the three professional educator pillars central to the development of quality teachers: (a) "professional commitment," (b) "professional practice," and (c) "professional impact" (MO-DESE, 2011, p. 9). All of Missouri's quality indicators and teaching standards are encompassed within these three overarching frames (MO-DESE, 2011, 2013)

Qualitative inquiry provided the strongest research design to discover the underpinnings of Marquardt's (2011) empowered and enabled learning phenomenon as it related to the teacher candidates. Qualitative methods provided the opportunity to collect 
rich data for analysis (Emerson et al., 1995). A variety of internal and external stakeholders (Marquardt, 2011) were included in the study to strengthen the research design and to lead to a better understanding of the central phenomenon.

\section{Participants}

A primary participant group, identified within Marquardt's (2011) people subsystem as customers, was earmarked for this phenomenological case study. That group was the front-line customers, graduate teacher candidates, who graduated from Midwest University's graduate deaf education teacher preparation program during the three year timeframe of the study or were still in the program at the end of the study. A secondary group of significant interest was the ultimate customers, young preschool children who were d/Deaf and received services through the program's preschool for young children who are d/DHH.

As previously stated, Midwest University's preschool program is housed within the program's speech-language-hearing clinic. All preschoolers who are d/DHH, ages 3 -6 years, are welcome into the program regardless of the degree of hearing loss, use or non-use of amplification, mode of communication, and the presence of additional disabilities. In part, program impact for this study was evaluated through families' perceptions of program impact on their preschoolers who were $\mathrm{d} / \mathrm{DHH}$ based on archival data gathered from Preschool Evaluation of Services forms across the three year span of the study. Other participant groups were also included to gain "the perspectives of those living in it" (Hatch, 2002, p. 7), thereby, increasing an understanding of the empowered and enabled learning phenomenon (Marquardt, 2011) as it related to the primary research participants. Purposive, nonrandom sampling was used to select participant groups 
because their input was believed to be important for answering the research questions (Fraenkel \& Wallen, 2009; Merriam, 2009).

In total, 44 people were invited to participate in this qualitative study or, in the case of the families of the preschoolers who were $\mathrm{d} / \mathrm{DHH}$, have their voices heard through archival documents. Gathering data from five different groups of participants enhanced the qualitative research design (Hatch, 2002). The participants encompassed both internal and external stakeholders whom Marquardt (2011) espoused were important for organizational learning. Table 1 depicts the breakdown of all participant groups.

Table 1

Breakdown of Stakeholder Participants

\begin{tabular}{lc}
\hline Internal Stakeholder Groups & Number of Participants \\
\hline Deaf education program faculty & $7^{*}$ \\
Full-time & 2 \\
Per course & $5^{*}$ \\
Teacher candidates & 9 \\
$1^{\text {st }}$ year & 7 \\
$2^{\text {nd }}$ year & 2 \\
Total & 15 \\
& \\
\hline External Stakeholder Groups & Number of Participants \\
\hline Preceptors & $11^{*}$ \\
Off-site practicum & 4 \\
Student teaching & $9 *$ \\
Deaf education graduates & 8 \\
Preschool family participants & 10 \\
Total & 29 \\
\hline Note. Two of the off-site practicum preceptors were also student teaching preceptors. They were only \\
counted once in the final total of possible participants. One of those preceptors was also per course \\
faculty. This person was counted once in each stakeholder group, yet, only once in total participants.
\end{tabular}


Seventeen possible participants were part of the primary participant group. They were the graduate teacher candidates of this program who either graduated from the program during the three year period of this study or were still enrolled as graduate teacher candidates during the spring 2014 semester, the semester in which the data for this study was collected. The 17 individuals who make up this primary participant group were further described and delineated within appropriate sub-categories in subsequent paragraphs.

The researcher categorized each teacher candidate according to the study's ending month and year, May 2014. Eight of the teacher candidates were teachers of students who were $\mathrm{d} / \mathrm{DHH}$. Those eight were external stakeholders and were referred to as deaf educators throughout the study. Two of the graduate teacher candidates graduated in May 2014. They were referred to as 2 nd year teacher candidates. The remaining seven were in their first year of the graduate deaf education teacher preparation program and were, therefore, referred to as 1 st year teacher candidates. Both 1st and 2nd year teacher candidates were included within the internal stakeholder group.

At the time of the study, Midwest University's graduate deaf education teacher preparation program offered three Master of Science degree options: (a) an accelerated master's option, (b) a traditional master's option, and (c) a track 2 option, primarily for international students, which would not lead to teacher certification. The accelerated masters' teacher candidates completed 13 hours of graduate credit while completing their undergraduate degree. Twelve of those credit hours counted for both their undergraduate and graduate degrees. 
Technically, the accelerated masters' students were not graduate students until they earned their bachelor degree. Following full graduate student status, those teacher candidates were slated to complete their coursework within one year, thus completing their undergraduate and graduate degrees within a 5-year period. Traditional masters' teacher candidates already had their bachelor's degree upon entering the graduate program. The accelerated and traditional masters' teacher candidates followed a consistent course sequence; therefore, the students completed their graduate coursework alongside their peers who were in their same cohort regardless of their accelerated or traditional masters' status.

Only those teacher candidates whose master degree included teacher certification were considered as possible participants in the study. This included the 17 teacher candidates mentioned previously. During the time of this study, there was only one graduate student enrolled in the track 2 option. Again, that individual was not included in the study as that degree does not lead to teacher certification. The 17 primary study participants graduated or were slated to graduate within the accelerated masters or traditional masters' options. Nearly half of those teacher candidates were accelerated masters' students and half traditional masters' students. Table 2 depicts the breakdown of the 17 primary study participants by category and masters' option.

Table 2

Primary Study Participants May 2014

\begin{tabular}{lccc}
\hline & Accelerated Masters & Traditional Masters & Total \\
\hline Deaf educators & 3 & 5 & 8 \\
$2^{\text {nd }}$ year teacher candidates & 0 & 2 & 2 \\
$1^{\text {st }}$ year teacher candidates & 5 & 2 & 7 \\
Totals & 8 & 9 & 17 \\
\hline
\end{tabular}


Homogeneous sampling (Fraenkel \& Wallen, 2009) occurred naturally within some groups. For example, deaf education teacher preparation programs tend to have more teacher candidates who are hearing than teacher candidates who are $\mathrm{d} / \mathrm{DHH}$ (Johnson, 2013). In addition, within the teaching profession as a whole, there are a greater number of females than males (Grant \& Murray, 2002). However, whenever possible, maximum variation samples were utilized to include diverse perspectives (Hatch, 2002). For this particular study, five of the possible participants were $d / \mathrm{DHH}$ and the remaining were hearing. By hearing status, the proportion of possible participants was $1: 8.8$. Therefore, 1 out of every 8.8 possible participants was $\mathrm{d} / \mathrm{DHH}$. All participants were female with the possible exception of the parents/families of the preschoolers. Because the Preschool Evaluation of Services form collected data anonymously, there was no way to know whether males or females completed the forms.

For this particular study, the best way to maximize sampling variation was to include, in as much as possible, all eligible participants as bounded by this one case and three year time span (Hatch, 2002). Following is a list of the internal stakeholder groups and the number within each group that were invited to participate: (a) seven deaf education program faculty, excluding the researcher, and (b) nine teacher candidates, seven 1st year and two 2nd year. One of the seven program faculty was also included within the external stakeholder group. Although this person was counted in both of these larger subgroupings, as internal and external stakeholders, this individual was counted only once in the final total of possible participants. The external stakeholders groups and the number within each group that were invited for participation are identified below. 
There were four off-site practicum and nine student teaching preceptors, bounded by this one case and three year period included within the external stakeholder group. Two of the off-site practicum preceptors were also student teaching preceptors during this three-year time frame. They were counted only once in the final total of possible participants. In addition, there were eight deaf educators who graduated from Midwest University's deaf education teacher preparation program in May 2012 or May 2013 who were also part of this external stakeholder group. As previously identified, the two teacher candidates who graduated in May 2014 were classified as 2nd year candidates since they had not yet taught as deaf educators. Thus, the 2 nd year candidates were internal stakeholders at the time of the study.

In addition to the aforementioned external stakeholders, there was one additional external group, the parents/families of the preschoolers who were $\mathrm{d} / \mathrm{DHH}$. There were up to 10 unique possible families of preschoolers who are $\mathrm{d} / \mathrm{DHH}$ enrolled in the program during the three-year bounded timeframe of this study. Unique indicated that there were a total of 10 preschoolers who were d/DHH in Midwest University's preschool program during the three year period. Therefore, 10 unique families were possible participants through archival documents.

\section{Internal Stakeholder Participants}

The 16 internal stakeholders were, in general, easily accessible to the researcher. This researcher had "insider" (Creswell, 2008, p. 219) status since she taught within this deaf education teacher preparation program for almost 23 years. During the proposal phase, the researcher received verbal permission from the primary gatekeepers (Creswell, 2008; Hatch, 2002), the program's department chair, clinic director, and the full-time 
deaf education teacher preparation faculty. Permission from the college dean was also obtained through the department chair. Opposition to this qualitative research study by any of the above individuals would have ended pursuit of this study.

Deaf education program faculty. Seven deaf education teacher preparation faculty were invited to participate in the study, excluding the researcher. Two of the seven were full-time faculty at Midwest University, one academic faculty and one clinical faculty. The full-time academic faculty was an associate professor and the fulltime clinical faculty member was a clinical associate professor. The other five deaf education program faculty were per course faculty who were hired, as needed, on a semester by semester basis.

The goal for surveying all education of the deaf program faculty members was to get the perspectives of all faculty members. The full-time faculty members were the best suited to explain the ways in which this deaf education teacher preparation program was designed to prepare teacher candidates who were empowered and enabled to learn to teach students who are d/DHH. It was also believed that the perspectives of the five per course faculty could add unique perspectives that would enhance understanding of the central phenomenon of study.

Lastly, the full-time deaf education teacher preparation program faculty member who was not surveyed was a clinical professor and the researcher of this study. She had taught at Midwest University for nearly 23 years. Although she was prelingually deafened in her right as a toddler, she has always considered herself to be hearing. She is White and female. More information about the researcher was explained in the positionality statement. 
Teacher candidates. All nine current teacher candidates were invited to participate. It was important to understand what current teacher candidates "believe, know, and think" (Fink, 2009, p. 11). Of the nine possible participants, all were female, eight were hearing and one was hard of hearing. Seven of the teacher candidates were 1st year students and the remaining two were 2 nd year teacher candidates. Special care was taken to ascertain that the teacher candidates understood that their participation was voluntary (Fraenkel \& Wallen, 2009; Hatch, 2002). In addition, all survey answers were received anonymously. If the researcher was able to identify teacher candidates due to demographic data or by their responses, their identity would be kept confidential. Furthermore, their involvement, in as much as possible, was distanced from the researcher's teaching responsibilities.

During academic year 2013-2014, none of the seven 1st year teacher candidates were taught by the researcher. However, since some of those students were taught by the researcher previously and all 1 st year teacher candidates would be taught by the researcher in subsequent semesters, their participation was requested through a survey instrument. It was believed that 1 st year teacher candidates may be more willing to report what they knew and perceived in survey format. In addition, 1st year teacher candidates were at the beginning of their practicum experiences. When they were surveyed, they were in their second of three on-site practicum placements with the preschool participants. The researcher believed it was important to gather information at different stages of program preparation. Also, surveys are important tools for gathering information from people. As Fink (2009) stated, "surveys are best when you need information directly from people about what they believe, know, and think" (p. 11). 
Both 2nd year teacher candidates were surveyed near the end of their student teaching experiences. The researcher was their university supervisor for student teaching. Anonymity of the 2 nd year teacher candidates was more difficult because there were only two students. In addition, one of the 2 nd year teacher candidates was hard of hearing and the other one was hearing. Their answers would be anonymous; however, if they were able to be identified through demographic data or their responses to the questions, their identity was kept confidential. It was believed that their input was important to include because they graduated from the program in May 2014 which made them the most recent program graduates. They had completed all coursework and practicum experiences within the current program design so they were surveyed during the final semester of their program preparation.

\section{External Stakeholder Participants}

All possible participants (a) four off-site practicum preceptors, (b) nine student teaching preceptors, (c) eight deaf educators, former graduates of this Midwest's teacher preparation program, and (d) 10 unique parents/families of preschoolers who were $\mathrm{d} / \mathrm{DHH}$ served in this program fit within this bounded case and three year time span (Savin-Baden \& Major, 2013). Only those preceptors still employed within the same school district were invited to participate. There was only one student teaching preceptor who was not asked to participate because that person no longer taught at the same school district in which she provided student teaching supervision for a Midwest University teacher candidate during this bounded study. As previously described, a couple of the preceptors served both as off-site practicum and student teaching preceptors during this bounded three year case study. 
External stakeholders were included in the study using various data collection methods. Both groups of preceptors, off-site practicum preceptors and student teaching preceptors were invited to be interviewed. The eight deaf education teachers, who had graduated from Midwest University during the bounded time of this study, were invited to participate in a survey. The voices of the preschoolers' parents/families were heard through archival documents collected through a Preschool Evaluation of Services form.

Off-site practicum preceptors. All four of the off-site practicum preceptors graduated from this Midwest University's deaf education teacher preparation program and were employed at public school districts within 45 minutes of the university. Fortyfive minutes between the practicum site and the university was the furthest distance considered for off-site practicum placements. This allowed teacher candidates reasonable access to both their practicum site and the university.

It was important to glean information from the off-site preceptors because they were the first to work with the graduate teacher candidates in the public school setting. Off-site practicum followed the teacher candidates' year of on-site practicum experiences in the program's preschool for students who are d/DHH. Their input was important to understanding preparation near the program's midpoint. The researcher had easy access to the off-site practicum preceptors because the off-site practicum preceptors were program graduates and because the researcher was responsible for off-site practicum placements. All off-site practicum preceptors who agreed to participate were interviewed. All of the off-site practicum preceptors were hearing and females.

Student teaching preceptors. There were nine individuals who were eligible to be participants. They were eligible because they were still employed in the school 
district in which they served as a student teaching preceptor for this university program within the study span of three years. Each of the student teaching preceptor participants was interviewed. It was important to include the voices of the student teaching preceptors because they provided the culminating externship experiences for Midwest University teacher candidates prior to graduation. Student teaching preceptors' perceptions of Marquardt's (2011) empowered and enabled learning phenomenon as it related to teacher candidates was critical to understanding this phenomenon.

The student teaching preceptors were located across two states and included deaf education teachers in public school districts as well as a School for the Deaf. Six of the nine student teaching preceptors served as student teaching preceptors at least twice for this deaf education teacher preparation program, some within this bounded time span. At the time of this study, the remaining three student teaching preceptors served in this capacity only once. In addition, four of the student teaching preceptors were program graduates of this deaf education teacher preparation program and five were not program graduates of this university. The researcher was responsible for making all student teaching placements; therefore, access to the nine student teaching preceptors was easily available to the researcher (Creswell, 2008). Of the nine possible student teaching preceptors, eight were hearing and one was deaf. All of the student teaching preceptors were females.

Deaf educators. Of the eight deaf educators invited to participate in this study, four graduated from this deaf education teacher preparation program in 2012 and four graduated in 2013. These deaf educators taught in five different states in the U.S., seven 
in public schools and one at a School for the Deaf. At the time of this study, these deaf educators were teaching in Colorado, Iowa, Kansas, Louisiana, and Missouri.

Gathering data from this deaf educator participant group could provide unique and important data. Since they were practicing deaf educators, they could reflect on their past program learning at this deaf education teacher preparation program. In addition, with these eight deaf educators teaching in five different states and in different types of schools, their data may shed light not only on how this teacher preparation program prepared them to teach students who were $\mathrm{d} / \mathrm{DHH}$, but how prepared they were to teach in different settings and across different states.

Although the deaf educators spanned across five states, there was easy access to these teachers. The researcher was able to contact them because of connections to the university, connections to deaf education program faculty, and through the use of social media, if needed. All of the deaf educators who participated in this study were surveyed. All were female and hearing.

\section{Parents/families of preschoolers who were d/DHH. Up to 10 unique}

parents/families were included in the study through archival documents. As previously mentioned, unique referred to 10 different preschoolers having participated in Midwest University's on-site, clinic preschool program during some part of this study's three year bounded timeframe. Parents/families were invited to complete the Preschool Evaluation of Services form during the course of the three year span of the study as clinic protocol. The gatekeeper, the clinic director, supplied access to the documents after IRB approval for archival documents. Depending on the number of semesters that the preschoolers attended, families were given multiple opportunities to complete the evaluation. There 
were six times during this three year time span that parents/families were asked to complete the Preschool Evaluation of Services form.

\section{Data Collection Instruments}

Creswell (2012) indicated that good qualitative case studies include a variety of types of data to acquire an in-depth understanding of the case. For this case study, data was gathered from five groups of participants: (a) deaf education program faculty, (b) teacher candidates, (c) preceptors, (d) deaf education graduates, and (e) preschool family participants through multiple sources: (a) interviews, (b) surveys, and (c) documents to get a more holistic perspective of the program and its teacher candidates (Savin-Baden \& Major, 2013). This phenomenological case study was bounded by three academic years, from August 2011 - May 2014 so data collected fit within this timeframe (Savin-Baden \& major, 2013). Interview, survey, and document protocols were developed to guide the data collection process (Creswell, 2008; Hatch, 2002). Collecting interview and survey data required participants' direct consent (Appendices A, B, \& C).

\section{Preceptor Interview Protocol}

Interviewing is one of the most frequently used methods of gathering qualitative data (Merriam, 2009). For this study, the interview included two distinct components; therefore, the interview protocol was divided into part one and part two (Appendices D \& E). Appendix D, part one of the interview, included the formal, qualitative interview questions (Hatch, 2002). Appendix E, part two of the interview, included the demographic interview items. The interview protocols were used with both sets of preceptors: (a) off-site practicum preceptors, and (c) student teaching preceptors. 
Both parts one and two of the interview included a place to record the interviewee category, the date interviewed, and an interview ID. This ID replaced the use of participant names. The key which linked participant names with their corresponding ID was kept in a separate and secure location from that of the data. The letter and numeric values were assigned based on the interviewee category and order interviewed respectively. Off-site practicum preceptors were labeled OPP 1 - OPP4. The student teaching preceptors were labeled STP1 - STP7. Again, two of the off-site practicum preceptors also served as student teaching preceptors during this span of three years. Offsite practicum was the focus of the four possible off-site practicum preceptor interviews. They may also decide to share information about their lived experiences as student teaching preceptors.

Formal, qualitative questions as described by Hatch (2002), also known as semistructured (Creswell, 2009; Merriam, 2009), include both structure and flexibility (Hatch, 2002). There was a specific order to the eight questions being asked (Appendix D) and all eight questions were asked of each preceptor, much like a standardized interview (Hatch, 2002). However, there were also follow-up questions asked that flowed from the individual preceptor's responses and added flexibility to delve deeper into the phenomenon studied.

The interview questions focused on collecting data from participants to help answer the research questions. The first three research questions centered on Marquardt's (2011) learning organizations people subsystem's empowered and enabled learning phenomenon. Specifically, the research questions targeted how this deaf education teacher preparation program empowered and enabled teacher candidates: (a) to 
learn commitment to the profession, (b) to learn proficiency in practice, and (c) to create learning impact in students who are d/DHH (MO-DESE, 2011). The final research question, how could the program improve to empower and enable the learning of teacher candidates who are prepared to teach students who are $\mathrm{d} / \mathrm{DHH}$, was designed to address program improvement.

Part two of the interview questions (Appendix E) was highly structured (Merriam, 2009) or standardized (Hatch, 2002; Merriam, 2009). These were the 12 specific demographic types of interview questions with primarily fixed multiple choice options. A couple of the questions provided an 'other' category so participants could fill in the blank with an answer that more accurately represented them. The participants' responses to these questions and qualitative question one provided a more in-depth understanding of the research participants, their schools, and the students who are $\mathrm{d} / \mathrm{DHH}$ whom they served. The 12 demographic types of interview questions were presented in paper format (Appendix E) to each preceptor at the end of the interview for completion. The interview form was collected prior to the researcher leaving the interview.

\section{Faculty Survey Protocol}

Midwest University's seven deaf education teacher preparation faculty, which excluded the researcher, were invited to participate in a survey. Two of the three fulltime deaf education teacher preparation program faculty were included, again, the researcher was excluded. The remaining five were per course faculty. The survey protocol mirrored the interview format almost verbatim (Appendix F). The qualitative questions were identical. There were only five demographic items included due to only including applicable questions. The qualitative questions primarily focused on collecting 
data from participants to help answer the research questions. The demographic items and qualitative question one helped the researcher to define the study participants.

There were two types of faculty IDs. The protocol included a place to record the faculty survey ID. This ID replaced the use of participant names. The letter and numeric values were assigned based on whether the faculty were full-time or per course and the order in which the survey responses were received. The deaf education teacher preparation full-time faculty were categorized as FF1 and FF2. The deaf education teacher preparation per course faculty were categorized as PCF1 - PCF5. There was also a place for the researcher to record the date the survey was sent and the date it was returned.

Even without the separate identification of full-time and per course faculty, it was almost impossible for the researcher to not know which full-time faculty completed the survey. Each full-time faculty member's role within this university's deaf education teacher preparation program was unique. The researcher was upfront with both faculty members that confidentiality could be assured, but not anonymity. Both faculty have a strong vested interest in deaf education, in general, and in this deaf education teacher preparation program, specifically. Therefore, they were willing to participate in this study. It was believed that it may be important to distinguish between the responses of the full-time and per course faculty in the analysis phase; therefore, that feature was added to the survey.

\section{Student Survey Protocol}

A survey was provided to the following program participants: (a) 1st year teacher candidates, (b) 2nd year teacher candidates, and (c) deaf education teachers, past program 
graduates who graduated in 2012 or 2013 . In general, the survey protocol mirrored the interview format, including the positioning of the questions (Appendix G). The qualitative questions were presented first followed by the close-ended demographic types of questions. The only difference in the qualitative questions was replacing the words teacher candidate with you for questions three, four, and five. These questions focused on the individuals' direct experiences with the program as former or current program teacher candidates. Nine demographic survey items were asked of this group due to only including applicable questions.

Each protocol included a place to record the student survey ID. This ID replaced the use of participant names. The letter and numeric values were assigned based on the student's category and the order in which the survey responses were received. Teacher candidates who were 1st year students were labeled 1TC1 - 1TC7. Those teacher candidates who completed the survey as 2nd year teacher candidates were labeled 2TC1 and 2TC2. The final group, deaf educators who were program graduates, was labeled DEd1 - DEd8. There was also a place for the researcher to record the date the survey was sent and the date it was returned.

The qualitative survey questions asked participants to describe their deaf education teacher preparation program experiences in learning professional (a) commitment, (b) proficiency in practice, and (c) their outcomes in creating learning impact with students who were d/DHH (MO-DESE, 2011). They were also specifically asked how the program could improve in preparing teacher candidates to teach students who are $\mathrm{d} / \mathrm{DHH}$. The demographic questions and qualitative question one provided the researcher with information to better describe the pool of research participants, the 
participants' schools in which they worked or were assigned for on-site practicum or student teaching, and the students who were $\mathrm{d} / \mathrm{DHH}$ whom they served.

\section{Program Documents Protocol}

The document protocol was designed to collect data focused on the research questions (Appendix H). The protocol was separated into six primary sections for ease of collecting evidence of empowered and enabled learning and contrary evidence to empowered and enabled learning (Marquardt, 2011) across the three professional educator pillars: (a) commitment, (b) proficiency in practice, and (c) learning impact (MO-DESE, 2011). Archival documents collected centered around both teacher candidates and program impact. Documents such as final student teaching evaluations and teaching philosophy statements were gathered within the three year time span of the study. Teacher candidates' teaching philosophy statements were created in the semester prior to engagement in student teaching.

\section{Preschool Evaluation of Services Document Protocol}

The archival Preschool Evaluation of Services documents were obtained to help measure program impact. The gatekeeper of the archival Preschool Evaluation of Services documents, the clinic director, granted permission for access and this process was also delineated in the IRB. The document protocol (Appendix I) included both quantitative and qualitative data collection items. The items were taken directly from the evaluation form. Only items believed to support answering the research questions, primarily program impact, were included in the protocol. Length of time the child was in the program and whether or not the form was completed by a parent or family member was also included to further define the population. 
The Preschool Evaluation of Services forms were de-identified. Clinic personnel gathered the protocols prior to the researcher gaining access in order to remove any identifying items. For example, although no form asked for identifying information such as a name, when completing the anonymous form, a parent may have referred to their child by using the child's first name. Those identifiers were removed prior to the researcher having access to the archival documents. In addition, the researcher made no attempt to determine the preschoolers, parents, or family members' identities.

\section{Data Collection Procedures}

This phenomenological case study of a deaf education teacher preparation program involved collecting data from a possible 44 participants out of a potential population of 45 individuals. One preceptor was not included because this individual was no longer at the district in which she taught when serving as a preceptor for Midwest University. As Creswell (2009) purported, "learning the meaning that participants hold" (p. 175) is critical in qualitative research. Using a variety of sources allowed the researcher to get a more holistic perspective of the program and its teacher candidates (Savin-Baden \& Major, 2013).

Thirty-three of the participants were invited to participate in an in-person interview or online survey. An additional person was invited to participate through the interview and online survey. Therefore, this person was invited to participate as both an internal and external stakeholder. She was an internal stakeholder as program per course faculty and an external stakeholder by serving as both an off-site practicum and student teaching preceptor during this three-year span of the study. 
The voices of up to 10 unique families of the preschoolers who received services during this bounded three-year study were heard through archival documents. Archival data from the families of the preschoolers was used to help determine program impact. Furthermore, unobtrusive documents and artifacts were gathered to provide insights into the phenomenon of study without interfering with the phenomenon within social contexts (Hatch, 2002).

If data saturation, "a state in which the researcher makes the subjective determination that new data will not provide any new information or insights for the developing categories" (Creswell, 2008, p. 646), was not achieved through the aforementioned sources the researcher would interview the four deaf educators who graduated in 2012. They were the study participant group labeled deaf educators and had the most field experience of the individuals within this group. These additional interviews could also be conducted as confirming and disconfirming sampling data (Creswell, 2008).

\section{Preceptor Interviews}

Approximately one hour interviews were conducted with off-site practicum and student teaching preceptors who agreed to participate in the study to build an understanding of Marquardt's (2011) empowered and enabled learning phenomenon through their perceptions of lived experiences (Hatch, 2002). Gathering input from these external stakeholders was important for understanding perspectives beyond the program walls. Collecting data through interviews allowed for flexibility in asking follow up questions that could lead to a deeper understanding of the phenomenon of study (Hatch, 
2002). Therefore, each of the preceptor's individual interviews was conducted in-person by the researcher. A total of 11 individuals were invited to participate in interviews.

Contacting preceptors was convenient because the researcher had established relationships with most of them. Six of the 11 preceptors were former graduates of the deaf education teacher preparation program. The researcher contacted most potential participants informally during the proposal phase to ascertain there was at least some interest in being interviewed. Since this case was bounded within a three year time frame, the number of participants was limited. If there was a trend for non-participation, the researcher may have needed to make adjustments to the study.

The researcher asked each participant for their preferred email for correspondence during the study. A consent form (Appendix A) was provided to each researcher through an email attachment following IRB approval. In the consent form, the researcher made it clear that their informal agreement to participate in the study was not a binding commitment to participate. It was within this email correspondence that an interview date was requested, for those willing to participate. It was also explained in the email that the researcher would bring a consent form to the interview. The researcher reviewed the consent form with each participant and acquired a formal signature for commitment to participate prior to beginning the interview. Their signed consent forms were kept in a folder separate from their interview protocols. For confidentiality, their interview protocols did not include their names.

Approximately one to two weeks prior to the interview, each preceptor to be interviewed was provided with general information about the type of interview questions. In addition, the interviewees were provided with one of the questions that they would be 
asked so they could give thought to that particular question prior to the interview. The question supplied was, "From your perspective, at the end of student teaching, what should a teacher candidate 'look like' who is ready to teach students who are d/DHH?". The other questions were not provided because the researcher wanted to avoid the possibility of the three overarching educator frames influencing their responses to the question provided in advance.

Each interview was audio-recorded. Interview locations were determined on an individual basis, primarily based on preceptor's preference for convenience, comfort, and privacy. In addition, the researcher requested a location with a good acoustic environment to better support communication and improved sound quality of the recording. One of the potential preceptors to be interviewed was deaf. In addition, the researcher has a profound, unilateral hearing loss and is challenged with listening in noisy environments. It was important that the quality of the recording allowed the researcher to transcribe the interview accurately. Most interviews occurred at the preceptor's school or at, or near, the hometown of the interviewee. Interview locations ranged from 20 minutes to $4 \frac{1}{2}$ hours from the university.

\section{Faculty and Student Surveys}

Surveys were provided to both internal and external stakeholders (Marquardt, 2011). The three internal stakeholder groups were (a) 1st year teacher candidates, (b) 2nd year teacher candidates, and (c) deaf education teacher preparation program faculty. The external stakeholder group was deaf educators, former program graduates who graduated in either 2012 or 2013. It was believed that it was important to gather information from current teacher candidates and program faculty. Survey responses received from external 
stakeholders had particular relevance to understanding perceptions beyond the program walls. The researcher had easy access to the email addresses of current teacher candidates and program faculty. The deaf educator group was also easy to locate due to this group being recent graduates of the program. Surveys were provided through the Internet using Qualtrics (Qualtrics, 2013).

A consent form was made available as an email attachment approximately one to two weeks prior to the survey being sent. Within the email, general information was included about the survey. As with the interview email, the survey email included one of the exact questions that would be asked on the survey to give the participant the chance to think about the question in advance. The question provided was, "From your perspective, at the end of student teaching, what should a teacher candidate 'look like' who is ready to teach students who are d/DHH?" (Appendices B \& C). The other questions were not provided because the researcher wanted to avoid the possibility of the three overarching educator frames influencing their responses to the question provided in advance.

Collection of survey data included a consideration of first language or native language (Mertens, 2015). For individuals who are Deaf and use ASL as their primary language, communicating in ASL over written English may be preferred. Therefore, the consent form included a sentence about accommodations being available. Furthermore, in a reminder recruitment email to a Deaf individual, this accommodation was made more explicit by indicating that their participation could be included through an in-person interview utilizing an ASL interpreter rather than in written format.

Although a consent form was sent to the survey participants in advance, the participant gave consent directly before beginning the survey items on Qualtrics. The 
consent information was again provided to the participants. Each participant had to select a box or radio button indicating that they were willing to participate. The selection of this button also allowed the participant to access the survey. By clicking into and completing the survey, the participants also acknowledged their willingness to participate.

\section{Program Documents}

Each document protocol indicated the type of document and document ID. The document ID replaced the use of participant names to protect participants' identities. There was also a place to record the date the document was retrieved. Archival clinic program documents were made available through the clinic director who served as the gatekeeper of the data and was covered through the IRB process. Document information needed for research purposes was transferred to the document protocol using only the document ID. The archival data collected was stored in a locked file cabinet in the researcher's office.

All 17 possible primary research participants (a) nine teacher candidates, seven 1st year and two 2nd year, and (b) eight deaf educators, former program teacher candidates within the 3-year span of this study, were requested to send their final student teaching evaluation and teaching philosophy statement to the researcher. Both the final student teaching evaluations and teaching philosophy statements were used as archival data in this case study. The request for these documents and the voluntary aspect of providing these documents were clearly outlined within the student recruitment email as well as within the consent form. The data collected from those documents were put on a program document protocol (Appendix H). Instead of the participant's name, a document ID was used. The data was kept in a locked file cabinet in the researcher's office. 


\section{Human Subjects Protection and Other Ethical Considerations.}

The researcher complied with all internal review board criteria for Midwest University where the study was located and University of Missouri, the university which would confer the researcher's doctoral degree, to ascertain that all human subjects were protected. Children are of high risk; therefore, protection of this group was a top priority. In addition, the researcher made every effort "to ensure that none of the information collected would embarrass or harm them [the participants]" (Fraenkel \& Wallen, 2009, p. 433).

Of additional consideration was confidentiality. With a low number of subjects, outliers were especially protected. Although, including a teacher candidate who did not graduate would provide integrity to the study by giving a more complete picture of the program and its participants (Creswell, 2008), issues of confidentiality were probable. Therefore, during the interview process, preceptors were asked to share information on the teacher candidates based on the teacher candidates who graduated. Also, because the researcher was the university supervisor of teacher candidates during student teaching in spring 2014, the voluntary aspect of participation was emphasized. Lastly, preceptors were given the opportunity to member check to ensure that what it recorded on their interview transcript was indeed what they intended to say (Creswell, 2012; Hatch, 2002).

\section{Data Analysis}

"Data analysis is a systematic search for meaning" (Hatch, 2002, p. 148). Application of rigorous data analysis techniques added to the overall credibility and trustworthiness of the findings (Patton, 1999). Following a postpositivist worldview perspective (Creswell, 2009; Hatch, 2002), this researcher utilized typological analysis as it provided more structure to data analysis. Initially, data was divided into categories 
based on the central phenomenon of study (Hatch, 2002), as defined within the professional educator frames of (a) commitment, (b) proficiency in practice, and (c) learning impact (MO-DESE, 2011). These educator frames embeded state and national competency areas for which Missouri teacher candidates and teachers should strive to be effective, even distinguished teachers (MO-DESE, 2011, 2013).

Preceptor interviews followed a two-step process prior to being sent to the interviewee for member checking. The transcription and transcript verification process involved the researcher and a graduate assistant. The graduate student was, primarily, responsible for the initial transcription. Each completed transcript was printed by the researcher. The verification process included listening to the interview and making changes directly on the hard copy of the transcript.

After completing the verification process, changes were made to the typed transcript prior to the transcript being sent to the interviewees who elected to member check their transcript. Member checking allowed interviewees to check the completed transcript to ascertain what was recorded in the transcript was indeed what they had intended to say (Creswell, 2012; Hatch, 2002). Member checking enhances the credibility of data collected (Mertens, 2015). Interviewees were allowed to strike through, delete, and add to their completed transcript to better reflect what they wanted to say. The member checked transcripts were the version used in the data analysis process.

Hatch (2002) believed analysis was about asking the right questions of the data. By beginning data analysis early in the process, the researcher was able to "shape the direction of future data collection...and...improve the quality of the research" (Hatch, 2002, p. 149). Careful observations of interviewee responses to the qualitative questions 
early within the interview process also helped shape the interview questions for subsequent interviews as well as the qualitative survey items.

Analysis included triangulation of data sources for findings validation (Creswell, 2008). Coding strategies such as placing parenthesis around data that indicates the ideal of what a teacher candidate should "look like" at the end of student teaching was printed directly on the transcripts and printed surveys. In addition, highlighting evidence, both positive and contrary, around the aforementioned educator frames (a) commitment, (b) proficiency in practice, and (c) learning impact, was included on the printed protocols. A different color was used to highlight the three different educator frames or pillars.

Typological analysis using these frames is a good starting point for a study of the empowered and enabled learning phenomenon (Marquardt, 2011). At this deductive stage, the whole phenomenon was divided into specific elements (Hatch, 2002). Data from each preceptor interview and each faculty and student survey were assimilated onto the appropriate summary sheet (Appendices J, K, \& L). Prior to data compilation, the summary sheets were printed on colored paper to easily delineate the participant groups. Summary sheets were designated by the participant type and data collection type. The color-coded paper facilitated triangulation of data across data sources (Creswell, 2008).

The data collected on the individual summary sheets focused on answering the research questions. Data entry included both positive and contrary evidence of empowered and enabled learning across the three educator frames. Also, within the transcript coding strategies such as starring powerful quotes and bolding words said with emphasis by the interviewee were used. Furthermore, powerful quotes were included in 
summary sheets with page and line numbers, as applicable which allowed for ease at verification to the original transcript, survey, or documents.

Following the grouping of data into typological categories, the researcher read data summary sheets multiple times to identify "patterns, relationships, [and] themes" (Hatch, 2002, p. 153). This search for meaning required inductive reasoning in which the researcher looked at the pieces to develop the whole. Patterns are those regularities that emerge from within the data and may be noted in different ways. Relationships describe how data is connected. Lastly, the themes are the broad statements of meaning that emerge.

Uncovering data that did not fit or that contradicted was also a part of the process. Careful scrutiny was required to provide an explanation that was satisfactory or the findings were adjusted to reflect this difference (Hatch, 2002). The researcher returned to the original data source and again read the transcripts and survey data to ascertain that the emerging themes were reflected in the original documents and something was not missed in translation to the summary sheets.

\section{Positionality of Researcher}

Researchers' philosophical beliefs influence qualitative research (Creswell, 2013). A postpositivist worldview suggests that researcher bias should be "controlled and not expressed in a study" (Creswell, 2013, p. 36). However, this researcher believes it is important to be upfront about her beliefs and biases related to this case study. The researcher has personal biases based on the researcher's lived experiences, and passion for and vested interest in this program of study.

As previously mentioned, the researcher is a clinical professor in this deaf education teacher preparation program. She has taught at Midwest University for nearly 
23 years. Throughout the researcher's tenure at the university, she has had the privilege of serving children as young as infants and toddlers who are $\mathrm{d} / \mathrm{DHH}$ and their families as well as preparing graduate teacher candidates who will be future teachers of students who are $\mathrm{d} / \mathrm{DHH}$ and many levels in between.

This researcher brings nearly 30 years of professional experience to this research study, almost 23 years of which have been within the field of deaf education. She has worked in a variety of settings and served in a variety of roles such as a (a) speech therapist, (b) teacher, and (c) cochlear implant educational consultant. Specifically, in the area of serving individuals who are d/DHH, the researcher has worked between 6-12 years in each of the following roles (a) early interventionist, (b) preschool teacher, (c) cochlear implant aural habilitation service provider, and (c) cochlear implant educational consultant for public school districts throughout the state of Missouri.

Throughout the researcher's years of experience, her philosophy in educating students who are d/DHH and her beliefs about parents with children who are d/DHH has emerged. The researcher believes that there is not one communication modality that fits all individuals who are d/DHH. Parents have choices regarding communication methodologies and technologies for their young children who are d/DHH. The researcher further believes that "having a positive belief set about parents and working from where the family is rather than where professionals believe the family should be...is a winning combination" (Wang \& Engler, 2011, p. 251).

Where Wang and Engler (2011) may have missed the mark was omission of the belief that parents want their children who are $\mathrm{d} / \mathrm{DHH}$ to be independent. This researcher believes in the potential of all children regardless of degree of hearing loss and presence 
of additional disabilities. Regardless of degree of hearing loss, too many individuals who are d/DHH are not reaching their full potential (Luckner, 2013; Moores, 2001; Spencer \& Marshark, 2010; The National Agenda, 2005; Traxler, 2000). Reaching one's full potential should be coupled with high expectations and omission of a good enough mentality.

The researcher has a profound, unilateral hearing loss as a result of an ototoxic drug that was administered when she contracted meningitis as a toddler. Because the researcher had meningitis in the early 1960s, it was not unusual that the hearing loss went undetected for a few years. In addition, the researcher's hearing acuity in the contralateral ear was within normal limits. No recommendation was made for a hearing aid. Preferential seating in the classroom and preservation of the "good" ear were the major recommendations made to the researcher's parents. Preservation of the good ear included wearing a swimming cap when swimming and being proactive in the event of ear infections or ear aches. As an adult, the researcher visited with an audiologist about the possibility of using a cross-aid; however, the researcher has not pursued that option.

The researcher's profound, unilateral hearing loss may have influenced her career path. It may also have sparked the researcher's passion for success in reaching one's full potential for all individuals who are $\mathrm{d} / \mathrm{DHH}$ regardless of degree of loss and unilateral or bilateral involvement. Regardless of the aforementioned biases, it is the people that matter the most to the researcher. It is the teacher candidates whom she serves and the outcomes of the ultimate customers, all children who are $\mathrm{d} / \mathrm{DHH}$ and their families that were of paramount importance. The researcher sought to explore the essence of Marquardt's (2011) empowered and enabled learning phenomenon as it applied to the 
participants. This was best discovered through a qualitative design (Creswell, 2008/2013).

\section{Trustworthiness of Research}

"The conventional criteria for trustworthiness are internal validity, external validity, reliability, and objectivity" (Lincoln \& Guba, 1985, p. 218). A lack of consensus existed among qualitative researchers on how to determine validity and reliability in qualitative inquiry (Fraenkel \& Wallen, 2009; Merriam, 2009) and whether it was even relevant to do so (Lincoln \& Guba, 1985; Wolcott, 1995). Likewise discrepancies existed on the terminology that should be used to represent the overarching concepts of validity and reliability and how those terms should be defined (Denzin \& Lincoln, 2005; Lincoln \& Guba, 1985; Merriam, 2009). For this study, the researcher used the following terminology as presented in Merriam (2009) to address the trustworthiness of the study: (a) credibility, (b) consistency, and (c) transferability. Following is a description of each term and how it was addressed in this study.

Credibility. Credibility is an important component of trustworthiness (Creswell, 2012). Credibility in sometimes used interchangeably with internal validity (Merriam, 2009). Lincoln and Guba (1985) believed that credibility should replace internal validity in qualitative research. They alleged that inquiry should be undertaken in a way that enhances the "probability that the findings will be found to be credible" (p. 296). Triangulation through corroboration among different people, triangulation of multiple data sources, as well as member checking are acceptable means for demonstrating credibility or validating findings (Creswell, 2012). 
To answer the research questions, the researcher included a variety of individuals' perspectives to understand empowered and enabled learning of teacher candidates (Marquardt, 2011). The participant groups invited within the bounded case of three years were (a) deaf education program faculty, (b) 1st and 2nd year teacher candidates, (d) offsite practicum preceptors, (e) student teaching preceptors, and (f) deaf educators who were former program graduates. Triangulation through corroboration occurred among the aforementioned groups. Archival documents, including the Preschool Evaluation of Services data was used to help answer research question three regarding creating learning impact in students who are $\mathrm{d} / \mathrm{DHH}$.

Triangulation of data sources included (a) interviews, (b) surveys, and (c) documents. Member checking (Creswell, 2012; Lincoln \& Guba, 1985; Merriam, 2009) was included at the level of requesting interview participants to read their transcripts to ascertain what was recorded was what they intended to say. In addition, the researcher provided as quick as possible turn-a-round time between interviews and transcription. Furthermore, the researcher read and reread data and data analysis to ensure that the findings were based on evidence accrued rather than inferences (Gay, Mills, \& Airasian, 2012).

Consistency. Consistency is sometimes used interchangeably with reliability (Merriam, 2009). "Reliability refers to the extent to which research findings can be replicated" (Merriam, 2009, p. 220). In this traditional sense, reliability poses a challenge for qualitative researchers. As Merriam (2009) reported, "human behavior is never static, nor is what many experience necessarily more reliable than what one person experiences" (p. 221). Lincholn and Guba (1985) coined the term consistency as it 
related to qualitative inquiry. Consistency of results needs to be sensible based on the data collected. The researcher provided detailed descriptions of data collection techniques and analysis to give the reader adequate information to judge dependability or consistency of findings.

Transferability. There is "seldom methodological justification for generalizing the findings" (Fraenkel \& Wallen, 2009, p. 432) of qualitative research. In quantitative research it is the randomization of the sample that allows inferential statics to be applied to the larger population (Eisner, 1991). However, by its very nature, purposive sampling is more common in qualitative inquiry (Fraenkel \& Wallen, 2009).

Transferability is sometimes used synonymously with external validity (Merriam, 2009). It is transferability that Lincoln and Guba (1985) and others (Eisner, 1991; Fraenkel \& Wallen, 2009; Merriam, 2009) highlight as being applicable to qualitative research. It is, however, at the discretion of the practitioner to determine applicability to their situation (Eisner, 1991; Stake, 2005). Giving a detailed description of the qualitative research setting is important for supporting transferability (Lincoln \& Guba, 1985; Merriam, 2009). The researcher provided a detailed description of the case, Midwest University's graduate, comprehensive, deaf education teacher preparation program.

This study had a unique opportunity to inform practice and educational leadership policies. This comprehensive, graduate, teacher preparation program attempted to fill two separate, but related needs (a) preparing teacher candidates who will teach students who are d/Deaf and hard of hearing, a critical area of need within U.S. public schools (American Association for Employment in Education, 2008; U.S. Department of 
Education, 2012) and (b) teaching children who are d/DHH, thus, assisting in filling that void. This research may provide considerations for transferability of program design regardless of communication philosophy (Fraenkel \& Wallen, 2009).

\section{Limitations and Assumptions}

\section{Limitations}

Qualitative research includes limitations (Creswell, 2008, 2009; Hatch, 2002, Merriam, 2009; Patton, 1999, Fraenkel \& Wallen, 2009). A significant limitation of this research is that it covers only one case. Patton (1999) also indicated limitations bounded by time, in this study, a three year period.

Although the researcher attempted to study all participants who fit within this bounded case, there were limitations regardless of the number who elected to participate. There were a disproportionate number of females as well as individuals who were hearing in this study. Of the 34 participants invited to participate, all were female and 30 were hearing. Similarities were noted within relevant teaching fields. Teachers in special education continue to be dominated by females (Rice \& Goessling, 2005). Furthermore, according to Moores (2001) deaf education teachers are predominately female and hearing. In addition to the aforementioned limitations, some participants may not be located and others may simply elect to not participate. Using a variety of data collection methods and triangulation of these data sources through rigorous analysis provided a validity cross-check of findings (Patton, 1999).

\section{Assumptions}

By its very nature, "qualitative work starts with the assumption that social settings are unique, dynamic, and complex" (Hatch, 2002, p. 9) and researchers approach study of the whole rather than pieces. As other researchers (Creswell, 2008; Emerson et al., 1995; 
Hatch, 2002; Savin-Baden \& Major, 2013), this researcher assumed there was merit in studying one case. Also, the researcher made the assumption that the participants were forthcoming and honest in their responses to questions through interviews and surveys. Philosophical assumptions are a part of qualitative research (Creswell, 2013). The following paragraphs depict the philosophical beliefs of the researcher and how that interfaces with the interpretive framework of the study (Creswell, 2013).

A postpositivist worldview perspective (Creswell, 2009/2013; Hatch, 2002) is primarily held by the researcher. Therefore, the researcher's ontological belief is that reality exists; however, that it may never be found in its entirety, only approximated (Creswell, 2013). Rigorous techniques were utilized in an effort to "capture close approximations of reality" (Hatch, 2002, p. 14). However, in contrast to a postpositivist paradigm, the researcher believed the voices of the participants influenced the validity of the research. Although data collection protocols were used, the researcher was attentive to the voice of the people thus allowing for an emergent design. Typological analysis was followed methodically.

Regardless of rigorous techniques, the researcher recognized interpretation of data was part of the process which interfaced within an interpretive-constructivist paradigm (Merriam, 2009). Furthermore, the researcher was cognizant of the possibility of multiple realities emerging from the data and emphasized to the research participants the importance of their lived experiences and perspectives in understanding the phenomenon of study. This extended into a constructivist worldview (Creswell, 2009; Merriam, 2009). 


\section{Summary}

Deaf education teachers were in demand in U.S. public schools (American Association for Employment in Education, 2008; U.S. Department of Education, 2012). Yet, deaf education teacher preparation programs were reportedly on the decline (Dolman, 2008; Johnson, 2013). Little was known about deaf education teacher preparation programs that existed and less was known about how those programs prepare teacher candidates while simultaneously serving young children who are d/DHH.

For this research, a phenomenon in the bounded case of a graduate, comprehensive, deaf education teacher preparation was explored through a qualitative, phenomenological case study (Savin-Baden \& Major, 2013). This research described the program and its people, namely, the teacher candidates through the phenomenon of study. The phenomenon studied, empowered and enabled learning, was drawn from Marquardt's (2011) learning organizations' people subsystem and guided the study. Empowered and enabled learning was defined through MO-DESE's overarching educator pillars: (a) commitment, (b) proficiency in practice, and (c) learning impact $(2011,2013)$. A variety of data collection methods were employed to increase understanding of the case (Creswell, 2009) (a) interviews, (b) surveys, and (c) documents. Aligned with the researchers primary postpositivist worldview perspective (Creswell, 2009; Hatch, 2002), this researcher used typological analysis to provide more structure to data analysis. This analysis allowed for deductive and inductive reasoning approaches resulting in emerging themes of the central phenomenon of study (Hatch, 2002). The researcher also recognized the interpretive-constructivist lens expressed by Merriam (2009). Research findings may inform deaf education leadership policies and practices. 


\section{CHAPTER FOUR}

\section{PRESENTATION OF FINDINGS}

Students who are $\mathrm{d} /$ Deaf or hard of hearing $(\mathrm{d} / \mathrm{DHH})$ account for only $1.2 \%$ of the U.S. school population of students with disabilities (U.S. Department of Education, 2012). Yet, a critical shortage of teachers of students who are $\mathrm{d} / \mathrm{DHH}$ has been documented within U.S. public schools (American Association for Employment in Education, 2008; U.S. Department of Education, 2012). Filling the need for teachers of students who are $\mathrm{d} /$ Deaf or hard of hearing $(\mathrm{d} / \mathrm{DHH})$ was further compromised because deaf education teacher preparation programs were reportedly on the decline (Johnson, 2013). Furthermore, if the deaf education teacher preparation programs identified at-risk indeed close, the Midwest will be hit the hardest (Benedict, Johnson, \& Antia, 2011).

Program disparity existed among some of the remaining deaf education teacher preparation programs due to communication philosophy (CED, 2013). Deaf education teacher preparation programs prepared teacher candidates based on their program philosophy. Furthermore, there are distinct differences among individuals who are d/DHH; they formed a diverse group (Johnson, 2013; Marschark et al, 2002; TyeMurray, 2009). It was imperative that the deaf education teacher preparation programs that remained help to fill the need for teachers of students who are d/DHH in U.S. public schools.

Little was known about the functioning of existing deaf education teacher preparation programs, especially programs espousing a comprehensive philosophy. Even less was known about deaf education teacher preparation programs that prepared teacher 
candidates while simultaneously serving young children who were d/DHH within their program walls.

This researcher conducted a qualitative, phenomenological case study (SavinBaden \& Major, 2013) of a graduate, comprehensive, deaf education teacher preparation program that included an on-site preschool program for young children who were d/DHH. The study was bounded by three academic years from fall 2011 - spring 2014. Marquardt's (2011) learning organization's people subsystem guided the investigation. The researcher explored how this one case prepared teacher candidates who were “empowered and enabled to learn" (Marquardt, 2011, p. 26).

Marquardt's (2011) empowered and enabled learning phenomenon was explored through Missouri's Department of Elementary and Secondary Education (MO-DESE) three professional education pillars deemed central to the development of quality teachers (MO-DESE, 2011, 2013). The pillars (a) commitment to the profession, (b) proficiency in practice, and (c) learning impact were used to frame the research questions. The researcher sought to answer the following research questions:

1. How does the program empower and enable teacher candidates to learn commitment to the deaf education profession?

2. How does the program empower and enable teacher candidates to learn proficiency in practice with students who are d/Deaf or hard of hearing?

3. How does the program empower and enable teacher candidates to create learning impact in students who are $\mathrm{d} /$ Deaf or hard of hearing?

4. How could the program improve to empower and enable the learning of teacher candidates who are prepared to teach students who are d/Deaf or hard of hearing? 
The presentation of findings were organized into three primary sections (a) data collection, (b) findings, and (c) summary. The data collection section began with a backdrop of the setting to provide a description of the program, its comprehensive philosophy and general information on coursework and practical experiences. Next, the pool of research participants were described along with the schools in which they served and the students who are $\mathrm{d} / \mathrm{DHH}$ whom they served. Then, data collection instruments and protocols were discussed. The next section, findings, was organized to answer the four research questions. The themes that emerged from the qualitative data were explored. A summary reviewing the findings concluded this chapter.

\section{Data Collection}

\section{Setting}

Midwest University was a pseudonym for the deaf education teacher preparation program of this phenomenological case study. Its deaf education teacher preparation program was designed to meet not only the required state standards, Birth-grade 12 (B12) Deaf and Hard of Hearing, but also the national certification standards outlined by CED and the Council on Exceptional Children (CED, 2013). Johnson (2013) reported that only half of the deaf education teacher preparation programs were designed to meet this higher, national certification standard.

Midwest University offered both an undergraduate and graduate degree in communication sciences and disorders with an emphasis in Education of the Deaf and Hard of Hearing. The graduate program consisted of $46-48$ credit hours depending on whether a student selected the graduate project or graduate thesis option. For teacher 
candidates in the accelerated master's option, 12 credit hours taken in their senior year of undergraduate studies counted for both their undergraduate and graduate degrees.

The graduate sequence was typically a five semester program which included one summer. Teacher candidates became eligible for initial teacher certification at the graduate level. Following was a description of this deaf education teacher preparation program's espoused philosophy placed within the framework of its position within this Midwest region thus further establishing the program setting.

Program philosophy. Midwest University was the only program in its state that embraced a comprehensive philosophy as identified by its national approval body, CED (CED, 2013). Three of its four primary bordering states had no deaf education teacher preparation program regardless of communication philosophy (Deaf Education Teacher Preparation Program, 2012). And as previously noted, some programs were in jeopardy of closing in the Midwest; this could result in a $40 \%$ decline in the Midwest's capacity to prepare future teachers of students who are d/DHH (Benedict, Johnson, \& Antia, 2011).

Because of its comprehensive philosophy, the program's intention was to prepare teacher candidates to teach students who are d/DHH regardless of the student's mode of communication. Therefore, Midwest University prepared teacher candidates through coursework and experiences designed to teach students who were d/DHH who use spoken language, sign language, or a combination of different methodologies. Following was an overview of Midwest University's courses and hands-on learning experiences. This overview reflected the experiences of the teacher candidates who were program students in this deaf education teacher preparation program sometime during the 
three-year span of this study. The 1st year teacher candidates had not yet completed all of the coursework or experiences.

Program coursework. All graduate teacher candidates' completed coursework specific to the area of $d /$ Deafness and educating students who were $d / D H H$ which were taught by deaf education preparation program faculty. Teacher candidates took two courses each in language development, speech development, and early intervention. They also took a course in reading, counseling, and instructional strategies. Program students were required to take research hours which culminated in a graduate project or thesis. There were other deafness related courses required if they were not taken at the undergraduate level.

The program also required coursework in sign language. The program offered a total of six sign language courses. There were four courses, three credit hours per course, of American Sign Language (ASL) and two courses, three credit hours each, of Conceptually Accurate Signed English (CASE). Although four of these courses, the first two ASL and both CASE, were required at the undergraduate level, only two courses were required for a teacher candidate entering the program at the graduate level. Teacher candidates were encouraged to take as many sign language courses as their schedules permitted, hopefully, at a minimum, four courses.

Teacher candidates were also required to take additional coursework for state teaching certification. Courses such as reading, math, and behavior management were required. In addition to coursework, teacher candidates engaged in a variety of hands-on learning experiences. Practicum was an integral component of this deaf education teacher preparation program. 
Program practicum and student teaching experiences. Teacher candidates completed their first year of practicum (fall, spring, and summer) within the on-site program's preschool for children who were d/DHH. In line with the program's philosophy, all young children who were d/DHH were welcomed. Preschoolers learned alongside their peers regardless of mode of communication, use or non-use of amplification, or the presence of additional disabilities (Wang et al., 2014).

This first practicum year of working with the preschoolers who were $\mathrm{d} / \mathrm{DHH}$ and the preschoolers' families was considered the cornerstone of practicum by full-time program faculty. Teacher candidates completed methodical, sequential practicum experiences that increased in expectations under the direction of the program's clinical associate professor who, in part, served as the classroom teacher and supervisor of the program. Each practicum course required at least three contact hours weekly in the preschool as well as weekly seminar meetings. The practicum experiences were frontloaded with individualized instruction and ongoing written feedback. Additionally, throughout the program, some graduate courses included additional learning experiences within the program's preschool and some teacher candidates participated in research within this setting as well.

Following the first year of on-site preschool practicum, teacher candidates were placed in the public school setting for one semester of practicum. The teacher candidates completed at least three hours of contact time weekly. They also attended a weekly seminar class with the program's clinical professor who was the university supervisor for off-site practicum and researcher of this study. 
The teacher candidates' culminating practical experience, student teaching evolved from an 11-week, full day placement at the beginning of this study to a 13-week, full day placement at the end of this study. Teacher candidates received ongoing written and oral feedback from the preceptors. Feedback was also provided by the same program faculty member who served as the university supervisor for their public school placements. The university supervisor observed and evaluated the teacher candidates at least twice during each placement.

All practicum and student teaching experiences included a mid-term self-study. During the mid-term evaluation, the team, in the case of off-site experiences, the teacher candidate, preceptor, and university supervisor met. The team discussed teacher candidate's strengths, areas for continued growth, and teacher candidate goals were set for the remainder of the semester. The process began with the teacher candidate's reflection on their practice.

The focus of Midwest University's comprehensive deaf education teacher preparation program was to prepare teacher candidates to educate the diverse population of learners who are d/DHH irrespective of communication modalities. The program's on-site preschool was a pivotal part of the program. Not only was Midwest University helping to fill the gap for teachers of students who are d/DHH in U.S. public schools through its deaf education teacher preparation program, it was simultaneously filling a community need by educating preschoolers who were $\mathrm{d} / \mathrm{DHH}$. The setting of this case study has been established. Next, the study participants were described as well as the schools and students they served.

\section{Participants}


All 17 potential Midwest University deaf education teacher preparation program teacher candidates who were accelerated or traditional masters graduate teacher candidates or who had already graduated during the three-year span of this study were invited to participate. These potential participants were the primary research participants and the focus of this phenomenological case study. However, as Marquardt (2011) espoused internal and external stakeholders were critical for organizational learning. The voices of 44 unique individuals or families were potential participants of this study.

Out of the 44 possible participants, 34 distinct individuals were invited to participate. The perspective of these 34 invited participants was believed important for answering the four research questions. The invited participants consisted of two internal stakeholder groups and two external stakeholder groups (Marquardt, 2011). Including a variety of participant groups strengthened the research design (Hatch, 2002).

To maximize sampling variation almost all possible participants across the threeyear span of the study were included (Hatch 2001). The preceptors invited to participate had to be employed by the same district or School for the Deaf in which they were teaching when they were a preceptor for this program during the three-years of the study. One of the potential preceptors was not invited to participate because she had taken an administrative position at a different school district.

The remaining 10 possible participants were not specifically invited to participate. They were included in the study primarily to assist in answering research question three on learning impact. Ten unique families were the total number of families who had a child who was d/DHH who attended the program's preschool sometime during the threeyear time span of this study. Their voices were heard through archival documents, 
specifically, the Preschool Evaluation of Services documents (PED1-PED26) that were collected as clinic program data during the course of the three-year span of this study. There was no way of knowing if all 10 unique preschool families participated in completing the evaluations because the data was collected anonymously.

At this point in the research, invited participant groups were organized around general categories: (a) program students, current and former, (b) program faculty, and (c) preceptors to assist in assimilating the findings. Of the invited participants, only two chose not to participate; therefore, 32 (94\%) of the invited participants agreed to participate. Of those invited, 28 completed participation in this study, thus, $87.5 \%$ of their voices were represented in the findings.

As previously mentioned, all general participant groups were well represented in this study. In addition, they were fairly equally represented across groups with actual percentage participation rates ranging from $81.8 \%-87.5 \%$. Of the 34 eligible study participants, only six individuals did not end up completing participation. Therefore, $82.4 \%$ of the entire eligible pool of invited participants was represented in the findings.

Each of the general categories of invited participants was further divided into subgroups. Program students included (a) 1st year teacher candidates, (b) 2nd year teacher candidates, and (c) deaf educators, former teacher candidates sometime during the threeyear span of this study. Program faculty included (a) full-time faculty, and (b) per course faculty. The final group of invited participants, preceptors, consisted of (a) off-site practicum preceptors and (b) student teaching preceptors. Table 3, presented on the next page, depicted the participation rate of all the participants. Preschool families were not included in the totals because the actual number of family participants was unknown. 
Table 3

Participation Rates of Study Participants

\begin{tabular}{|c|c|c|c|c|c|}
\hline $\begin{array}{l}\text { Invited } \\
\text { Participant } \\
\text { Groups } \\
\end{array}$ & $\begin{array}{r}\text { \# Invited } \\
\text { Participants }\end{array}$ & $\begin{array}{l}\text { \# Agreed to } \\
\text { Participate }\end{array}$ & $\begin{array}{r}\text { \# Actual } \\
\text { Participants }\end{array}$ & $\begin{array}{r}\text { Percentage of } \\
\text { Agreed } \\
\text { Participation } \\
\end{array}$ & $\begin{array}{r}\text { Percentage of } \\
\text { Actual } \\
\text { Participation } \\
\end{array}$ \\
\hline Students & 17 & 16 & 14 & $94.1 \%$ & $87.5 \%$ \\
\hline 1st Year & 7 & 7 & 6 & $100.0 \%$ & $85.7 \%$ \\
\hline 2nd Year & 2 & 2 & 1 & $100.0 \%$ & $50.0 \%$ \\
\hline Deaf Ed. & 8 & 7 & 7 & $87.5 \%$ & $100.0 \%$ \\
\hline Faculty & 7 & 6 & 5 & $85.7 \%$ & $83.3 \%$ \\
\hline Full-Time & 2 & 2 & 2 & $100.0 \%$ & $100.0 \%$ \\
\hline Per Course & $* 5$ & $* 4$ & 3 & $80.0 \%$ & $60.0 \%$ \\
\hline Preceptors & 11 & 11 & 9 & $100.0 \%$ & $81.8 \%$ \\
\hline \multicolumn{6}{|l|}{ Off-site } \\
\hline Practicum & $* 4$ & $* 4$ & 4 & $100.0 \%$ & $100.0 \%$ \\
\hline \multicolumn{6}{|l|}{ Student } \\
\hline Teaching & 7 & 7 & $* * 5$ & $100.0 \%$ & $71.4 \%$ \\
\hline \multicolumn{6}{|l|}{ Preschool } \\
\hline \multicolumn{6}{|l|}{ Family } \\
\hline Participants & $\mathrm{N} / \mathrm{A}$ & N/A & Up to 10 & Unknown & Unknown \\
\hline Total & $* 34$ & $* 32$ & 28 & $94.1 \%$ & $87.5 \%$ \\
\hline $\begin{array}{l}\text { Note. }{ }^{*} \text { One uni } \\
\text { site practicum } \\
\text { **All } 11 \text { invite } \\
\text { were transcribe } \\
\text { checking proce } \\
\text { the findings. }\end{array}$ & $\begin{array}{l}\text { e participant } \\
\text { peeptor. This i } \\
\text { preceptors agr } \\
\text { Two of the s } \\
\text { so they were }\end{array}$ & $\begin{array}{l}\text { eed to particip } \\
\text { ividual was on } \\
\text { d to be intervi } \\
\text { en student teac } \\
\text { t counted as ac }\end{array}$ & $\begin{array}{l}\text { ate as both per } \\
\text { ly counted onc } \\
\text { wed and were } \\
\text { hing preceptor } \\
\text { tual participant }\end{array}$ & $\begin{array}{l}\text { ourse program } \\
\text { in the total pool } \\
\text { nterviewed. All } \\
\text { did not complet } \\
\text {, nor was their d }\end{array}$ & $\begin{array}{l}\text { culty and off- } \\
\text { of participants. } \\
1 \text { interviews } \\
\text { the member } \\
\text { ta included in }\end{array}$ \\
\hline
\end{tabular}


Demographics. Each general participant group included one individual who was deaf or hard of hearing. Therefore, there were three (10.7\%) known individuals who were $\mathrm{d} / \mathrm{DHH}$ in the participant group. There was one potential preschool family participant who was deaf. Because the archival Preschool Evaluation of Services documents were completed anonymously, there was no way to know if that particular parent participated. Two of the aforementioned individuals self-identified as deaf and one identified as hard of hearing. To better protect participants' identities, the researcher did not specify the exact group or sub-group to which each individual belonged. The remainder of the actual participants, 25 out of $28(89.3 \%)$, were hearing. There was also one individual who was a CODA, child of deaf adults. This individual was hearing and, therefore, included in that percentage.

As noted above with hearing status, gender, race and ethnicity were also heavily weighted in one category. All participants in this study, except for possibly the preschool family participants were females. The majority of the participant pool, 24 out of 28 , $(85.7 \%)$ was White. Of the remaining four participants, two were Asian, one was Hispanic or Latino, and one was American Indian, Caucasian, and Hispanic. Again, the researcher did not specify the sub-groups to which each participant belonged to protect identities.

Fourteen program students (a) 1st year teacher candidates, a 2nd year teacher candidate, and (c) deaf educators participated in the study. The program students were equally divided on their master's option during graduate school, accelerated or traditional. Seven of the program participants were accelerated master's students and seven were traditional master's students. 
There were six current teacher candidates who were still program students at the time of reporting the findings. All six of those students had completed a bachelor degree and were working toward a master's degree at the time of the study. The remaining eight individuals were graduates of the program holding a master's degree. Four of them were completing their second year of teaching, three were competing their first year of teaching, and one had just graduated.

The five program faculty participants formed an eclectic group. One person had a Ph.D. Another program faculty had two master degrees, one degree in the area of deaf education and the other degree in the area of vocational rehabilitation. Another program faculty had a master's degree in the area of deaf education and was nationally certified as an interpreter. For the final two program faculty, one had a degree in the area of deaf education with additional university credits. The other participant had a master's degree in counseling. As a group, the program faculty represented combined years of teaching at Midwest University of at least 25 years with a range from 1-2 years up to 11-15 years. The faculty's years of experience teaching students who were d/DHH ranged from 0-17 years. The 17 years spanned across early childhood through high school.

The preceptor participants had a total combined minimum years of teaching experience of over 101 years. Their years of experience ranged from 3-5 years up to over 20 years teaching students who were $\mathrm{d} / \mathrm{DHH}$ and included teaching in public schools and a School for the Deaf. They also had various types of teaching positions ranging from an itinerant position covering multiple rural districts to a self-contained room in a School for the Deaf and a variety of other placement type options. 
Out of the nine preceptors, one (1.1\%) held a bachelor's degree, the other eight (88.9\%) had master degrees with four of them having university credit beyond the master's degree. One of the teachers was nationally board certified. Another preceptor was a certified interpreter as well as a teacher. All four (100\%) off-site practicum preceptors were program graduates. Two out of the five (40\%) student teaching preceptors were program graduates; three $(60 \%)$ were not program graduates. Next, the researcher provided information about the schools and the students who were $\mathrm{d} / \mathrm{DHH}$ whom they served.

Description of schools. Eight of the nine preceptors were teaching students who were $\mathrm{d} / \mathrm{DHH}$ at the time of the study. The other preceptor was serving as a K-5 academic coach for teachers of students who were d/DHH. Seven out of the nine (77.8\%) preceptors were employed within the public school system; two (22.2\%) were employed at a School for the Deaf. The teachers taught in two different states within the Midwest. The specific states were not mentioned to protect the study participants' identities.

The U.S. National Center for Education Statistics divided school districts by locale (National Center for Education Statistics, 2012). There were four general locale categories in which public school districts placed: (a) "rural," (b) "town," (c) "suburban," and (d) "city" (National Center for Education Statistics, 2012, p. 1). Each category was further divided into three specific categories. For the purposes of this study, the general categories were used with the same categories used for both public schools and Schools for the Deaf. Over the three year span of this study, Midwest University preceptors covered three $(75.0 \%)$ of the four general locale categories. 
Five out of the nine (55.6\%) preceptors taught in rural school districts. One of those teachers covered at least four different rural districts. Two (22.2\%) preceptors taught in suburban public school districts. The final two (22.2\%) taught in a city at a School for the Deaf. Additionally, the on-site preschool program, also a practicum site, fell within the city category. The town locale is the only district type that was not covered within the three-year span of this study. All teacher candidates had at least one rural and one city hands-on teaching experience with students who were d/DHH. Not all teacher candidates had a suburban teaching experience.

The seven deaf educators were also teaching at the time of this case study. Of the seven deaf educators, six (85.7\%) were employed in public schools as teachers of students who were $\mathrm{d} / \mathrm{DHH}$. The remaining deaf educator (14.3\%) taught at a School for the Deaf. Their schools were located across four different states within the U.S. (a) Colorado, (b) Iowa, (c) Missouri, and (d) Louisiana. The diverse school settings have been established. Next, the demographics of the population of students who are $\mathrm{d} / \mathrm{DHH}$ whom they served followed.

Description of students who were d/DHH. The students who were d/DHH were served by the preceptors, deaf educators, and the clinical associate professor of the program's preschool at the time that the study data was collected, spring 2014. Although the student demographics did not exactly match the students who were $\mathrm{d} / \mathrm{DHH}$ that were served within the entire three-year period of this study, the researcher believed that the pool was representative of a typical time frame.

Only preceptors who were still working in the same district at the time of the study as when they were a preceptor during the course of this study were included in the 
study. One preceptor was not invited to participate because this person had changed districts. The researcher thought that the student population had a higher probability of changing if the preceptor changed districts rather than remaining in the same district. It was believed that the findings could be better understood within the context of the students who were d/DHH who were being served by study participants. Lastly, and most importantly, the researcher did not want to breakdown the students who were d/DHH into smaller groups: (a) preceptor's students, (b) deaf educators' students, and (c) program preschool students to protect the identities of the students who were $\mathrm{d} / \mathrm{DHH}$.

The majority (64.4\%) of the students who were $\mathrm{d} / \mathrm{DHH}$ served by study participants were White. The next largest race and ethnic groups of students were Hispanic or Latino (18.8\%) and African American (8.9\%). The number of students in the other ethnic categories was too small to display at the one-tenth percentage level. There were, however, students who fell within the following categories: (a) Asian, (b) American Indian or Alaska Native, and (c) other. The race or ethnicity of the four students who fell within the 'other' category were not specifically noted to protect their identities. There was no student who was d/DHH that fell within the Native Hawaiian or other Pacific Islander category.

There were between 93 and 105 students who were d/DHH served during spring 2014 when the study data was collected. There was some variation in the reporting of data by participants; hence, the number of students counted in any specific category differed. The differences in participant numbers were reflected on the subsequent page in Table 4. 
Table 4

Students who are d/DHH Served by Project Participants

Number of Students Percentage of Students

Additional Disabilities

No

75

$71.4 \%$

Yes

30

$28.6 \%$

Total Number of Students

*105

Primary Mode of Communication

ASL

16

$16.7 \%$

Sign lang. with spoken lang.

27

$28.1 \%$

Spoken language

53

$55.2 \%$

Total Number of Students

*96

Amplification

Bilateral CI

16

$17.2 \%$

One CI only

6

$6.5 \%$

$\mathrm{CI}+\mathrm{HA}$

8

$8.6 \%$

Bilateral HA

42

$45.2 \%$

One HA only

9

$9.7 \%$

Bone Conduction Aid

3

$3.2 \%$

No amplification

8

$8.6 \%$

Other

1

$1.1 \%$

Total Number of Students

$* 93$

Note. *Data for total number of students who were $\mathrm{d} / \mathrm{DHH}$ per category varied based on participants' responses to demographic items. 
The data reported in Table 4 indicated that approximately $70 \%$ of the students had no additional disabilities and approximately $30 \%$ were reported to have additional disabilities. During the interview, there was some question by some preceptors on whether to report a student as having additional disabilities if they were not officially diagnosed as such. There was no way of knowing whether participants surveyed had a similar consideration or if they did how this was reflected in the numbers reported in Table 4.

The primary mode of communication category yielded diversity as well. Over half $(55.2 \%)$ of the students used only spoken language. However, within the pool of schools, only two school environments were strictly, at the time of this study, serving only students who were d/DHH who communicated through listening and spoken language. Also within this pool, there were two distinct school environments that, at the time of this study, were only teaching students whose primary mode of communication was sign language with spoken language. These student groups made up $28.1 \%$ of the population of students who were d/DHH served by the participants in this study.

The remaining 12 schools (over 70\%) were equally divided regarding the mixed communication modalities of the students. Six schools served students who represented two different primary modes of communication categories and six schools included all three primary modes of communication categories among the students who were $\mathrm{d} / \mathrm{DHH}$. In essence, most teachers were serving in schools with students whose primary mode of communication encompassed at least two, if not all of the primary communication modality categories. 
Diversity within the area of amplification was also apparent. Out of the 93 students who were d/DHH, 51 (54.8\%) of the students used only hearing aid (HA) technology, unilateral or bilateral. There were $22(23.7 \%)$ students who utilized cochlear implant (CI) technology, unilateral or bilateral. There were two groups who represented eight (8.6\%) students each. One group used one CI and one HA. The other group used no amplification. Of the remaining students, three (3.2\%) utilized bone conduction technology and one (1.1\%) fell within the 'other' category.

\section{Instruments and Protocols}

Surveys were sent electronically to teacher candidates, current and former, and program faculty who agreed to participate in this study. The surveys were disseminated through Qualtrics (Qualtrics, 2013). Survey distribution was non-anonymous; however, the participants' responses were received anonymously. Within Qualtrics, completed surveys were assigned a number; their name or email addresses were not included.

Because the researcher was a program faculty member at the time of this study, extra care was taken to make sure that research participants understood that their participation was completely voluntary. Therefore, at the most, one participant may have received only one reminder recruitment email and only one reminder for completion email. There was an exception. The exception was when research participants contacted the researcher specifically. In that case, the researcher followed up at the request of the participant.

Student surveys were sent to the 16 program students who agreed to participate in the research. Of this group, there were (a) seven 1st year teacher candidates (1TC11TC7), (b) two 2nd year teacher candidates (2TC1 \& 2TC2), and (c) seven deaf 
educators (DEd1-DEd7) who were former program teacher candidates. The seven deaf educators were external stakeholders at the time of the study. The survey consisted of qualitative questions and some close-ended demographic items (Appendix G). Fourteen (87.5\%) of the program students completed the survey.

Faculty surveys were sent to the six faculty members who agreed to participate in the study. Of this group, there were (a) two full-time program faculty (FF1 \& FF2), and (b) four per course program faculty (PCF1-PCF4). The qualitative questions on the survey nearly mirrored the questions on the student survey (Appendix, E). The demographic items were slightly changed. There was no need to determine the students who were $\mathrm{d} / \mathrm{DHH}$ that they served. Most $(80.0 \%)$ of the faculty who participated in the survey did not directly serve students who were d/DHH. Five (83.3\%) faculty actually participated in the survey. The faculty member who did not participate in the survey had participated in and completed the interview process as a preceptor. Therefore, in reality, the voices of $87.5 \%$ of the faculty were reflected in the data.

Interviews were conducted with four off-site practicum preceptors (OPP1-OPP4) and seven student teaching preceptors (STP1-STP7). Even though three of the practicum preceptors also served as student teaching preceptors at some time for this program, they were only counted once in the preceptor category as off-site practicum preceptors. There was $100 \%$ agreement by both preceptor groups to participate in the interview process. Nine of the 11 invited preceptor participants (81.8\%) completed the interview process (OPP1, OPP2, OPP3, OPP4, STP1, STP2, STP4, STP6, STP7).

The interview process included being interviewed by the researcher and member checking the transcription if they wanted to do so. Member checking was included in this 
study to ascertain that what was transcribed was indeed what the interviewee wanted to say. Of the 11 participants interviewed, 10 chose to participate in the member checking process. One opted out of the member checking process and granted permission for the researcher to use the transcript. Two (18.2\%) of the 11 interviewed did not follow through with the member checking process (STP3, STP5). Therefore, those two student teaching preceptors' transcripts were not included in the study nor were those preceptors counted in the total of actual participants. Table 5 depicted the participants' ID codes by participant groups and data collection instruments.

Table 5

Participant ID Codes and Collection Instruments

\begin{tabular}{lrr}
\hline Participant & ID Code & Instrument Type \\
\hline Program Students (current \& former) & & \\
$1^{\text {st }}$ Year Teacher Candidates & 1TC1-ITC6 & Student Survey \\
$2^{\text {nd }}$ Year Teacher Candidates & 2TC1 & Student Survey \\
Deaf Education Teacher Candidates & DEd1-DEd7 & Student Survey \\
Program Faculty & & \\
Full-time & FF1 \& FF2 & Faculty Survey \\
Per course & PCF1-PCF3 & Faculty Survey \\
Preceptors & & \\
Off-site Practicum & OPP1-OPP4 & Interview \\
Student Teaching & $*$ STP1,2,4,6,7 & Interview \\
Preschool Family Archival Documents & PED1-PED26 & Archival Documents
\end{tabular}

Note. *All 11 invited preceptors agreed to be interviewed and were interviewed. All interviews were transcribed and verified. Two of the 11 preceptors did not complete member checking. 
Documents, student teaching final evaluations and teaching philosophy statements, were requested of nine individuals. They were the (a) two 2 nd year teacher candidates, and (b) seven former teacher candidates who were deaf educators who had already completed those program milestones. Of the 18 possible documents, only two student teaching final evaluations and one teaching philosophy statement were sent to the researcher which indicated a 16\% return rate. Both student teaching final evaluations were completed by two different supervisors, in essence, there were four documents. However, since each set was related to only one teacher candidate, the evaluations that were completed for the same student were given an A and B distinction (STED1A \& STED1B; STED2A \& STED2B).

There were several communications from participants to the researcher expressing challenges with forwarding the requested documents to the researcher and a desire to meet this request. The most common challenges expressed were difficulty in finding the documents and not receiving their completed portfolio from the program that included those specific program documents. The researcher thanked the program students for their efforts and suggested they discontinue their search. There were no separate reminder emails sent about the above documents. The reminder was included within the survey. The documents that were received were used as evidence to confirm or disconfirm the findings generated through the interviews and surveys.

Twenty-six Preschool Evaluation of Services survey documents (PED1-PED26) were received during the course of the three-year span of this study. This survey was an ongoing part of the program clinic's protocol for evaluation of services. The documents were completed anonymously; however, whether the person was a parent or other family 
member was requested as well as the length of service the child who was $\mathrm{d} / \mathrm{DHH}$ was in the preschool program was also collected. Out of the 26 archival documents at least 21 (80.7\%) documents were completed by a parent, $3(11.5 \%)$ were completed by a family member, and $2(7.6 \%)$ were unknown. The latter two appeared to miss the whole back side of the survey, the page that included the request for this data. Table 6 depicted the archival program documents used in the study.

Table 6

Archival Data Collection

\begin{tabular}{lrrr}
\hline & $\begin{array}{r}\text { \# Possible } \\
\text { Documents }\end{array}$ & $\begin{array}{r}\text { Total \# of Documents } \\
\text { Received }\end{array}$ & Percentage \\
\hline Student Teaching & 9 & & \\
Evaluation (STED) & & 2 & $2.2 \%$ \\
Teaching Philosophy & 9 & 1 & $11.1 \%$ \\
Document (TPD) & & & \\
Preschool Evaluation & & & $*$ Unknown \\
Document (PED) & $*$ Unknown & 26 &
\end{tabular}

Note. ${ }^{*}$ A total of 10 unique preschool families had a child in the program preschool. Data was collected anonymously at six points throughout the three-year span of this study; therefore, the actual number of families voices heard and the possible documents and percentage of collection were unknown.

For this study, data was collected from a variety of sources: (a) interviews, (b) surveys, and (c) documents and a variety of participants: (a) program students, (b) program faculty, and (c) preceptors. Having multiple data sources and participant groups enhanced the quality of the research design (Hatch, 2002). Transferability of the findings will be determined by the reader through the rich description of the context of the study provided (Mertens, 2015). 
Midwest University was the pseudonym used for this comprehensive, graduate deaf education teacher preparation program located in the Midwest. Midwest University prepared teacher candidates for state teaching certification, B-12 Deaf and Hard of Hearing, and national approval through CED (CED, 2013). It was the only comprehensive program in the state (Deaf Education Teacher Preparation Programs, 2012). At the time of the study, there were no deaf education teacher preparation programs in three of its four primary bordering states.

The pool of actual participants represented $82.4 \%$ of the voices of the entire pool of possible participants. A majority of the participants were female, hearing, White, and graduated or will graduate from this deaf education teacher preparation program. There were at least three $(10.7 \%)$ research participants who were d/DHH. Program faculty formed an eclectic group. The school environments in which the participants served were diverse by school type, school locale, and position type. The preceptors exhibited an abundance of collective experience, over 100 years, teaching students who were d/DHH. The group of students who were d/DHH showed diversity in mode of communication, amplification type, and whether the students had additional conditions or disabilities.

\section{Findings}

Marquardt's (2011) empowered and enabled learning phenomenon within his people subsystem was used to guide this research. MO-DESE's three educator pillars: (a) commitment to the profession, (b) proficiency in practice and, (c) learning impact were deemed paramount to the development of quality teachers (MO-DESE, 2011, 2013). Therefore, MO-DESE's pillars were used to frame the research questions to explore the empowered and enabled learning (Marquardt, 2011) of the program's teacher candidates. 
The more participants with whom a particular theme resonated, the more prevalent that theme was. Themes were presented in order of prevalence. Some themes were labeled as dominant themes, while other themes were labeled as emerging themes depending on how prevalent the themes were. The data had to echo a particular theme from at least 8-12 participants for it to be recognized as a dominant theme. An emerging theme was any theme that resonated with 5-7 participants. Therefore, emerging themes resonated with approximately $15 \%-25 \%$ of the participants. Dominant themes resonated with greater than $25 \%$ of the participants. Any data that represented the voices of only one to four participants was not considered a theme. Figure 1 on the subsequent page depicted the dominant and emerging themes that emerged from this study.

As previously stated, program archival documents, namely, student teaching final evaluations and teaching philosophy statements were used to confirm or disconfirm the findings. In addition, although the preschool archival documents (PED1-PED26) were intended to be used to assist in answering research question three on learning impact, they were instead used to confirm or disconfirm the findings for research question three. This change in findings presentation resulted from the inability to ascertain how many of the 10 possible unique preschool family participants participated and at what level they participated. For example, there was no way of knowing if the supporting data which lead to a dominant theme, was actually due to counting the same family for similar qualitative responses as separate families. In essence, the researcher was concerned that using the data as if each of the 26 documents represented a different individual would skew the findings. 
Commitment Proficiency in Practice Learning Impact

\begin{tabular}{|c|c|c|}
\hline Dominant Themes: & Dominant Themes: & Dominant Themes: \\
\hline $\begin{array}{l}\text { Embracing } \\
\text { Diversity of } \\
\text { d/Deafness }\end{array}$ & $\begin{array}{l}\text { Differentiated } \\
\text { Instruction } \\
\text { How to Teach }\end{array}$ & \\
\hline Passionate Caring & Preparedness & \\
\hline $\begin{array}{l}\text { Above and Beyond } \\
\text { Expectations }\end{array}$ & Flexibility & \\
\hline $\begin{array}{l}\text { Dedication to } \\
\text { Learning }\end{array}$ & $\begin{array}{c}\text { Building } \\
\text { Competence } \\
\text { through Practice } \\
\text { Knowledge through } \\
\text { Coursework }\end{array}$ & \\
\hline Emerging Themes: & Emerging Themes: & Emerging Themes: \\
\hline $\begin{array}{l}\text { Learning to } \\
\text { Collaborate }\end{array}$ & $\begin{array}{l}\text { Critical Thinking } \\
\text { Evidence-based } \\
\text { Teaching } \\
\text { Ongoing Feedback }\end{array}$ & $\begin{array}{l}\text { Meeting Diverse } \\
\text { Learners' Needs } \\
\text { Collaborating with } \\
\text { Educators } \\
\text { Teaching Beyond } \\
\text { Academics } \\
\text { Connecting with } \\
\text { and Engaging } \\
\text { Students }\end{array}$ \\
\hline
\end{tabular}

Foundation of this Deaf Education Teacher Preparation Program

Embracing Diversity of d/Deafness \& Differentiated Instruction

Meeting Diverse Learners' Needs in Diverse Contexts

Figure 1. MO-DESE educator pillars (MO-DESE, 2011, 2013) and this study's themes. 


\section{Research Question One}

How does the program empower and enable teacher candidates to learn commitment to the deaf education profession?

Commitment to the profession is the first of Missouri's educator pillars considered paramount to the development of quality teachers (MO-DESE, 2011, 2013). According to MO-DESE $(2011,2013)$, commitment was rooted in professionalism, lifelong learning, and high expectations reflected through caring teachers who were determined to assist every student reach his or her full potential. To determine the program's influence on teacher candidate's commitment, the researcher asked about the program's role in the development of teacher candidates' commitment to the deaf education profession.

In the area of commitment to the profession, the following five dominant themes emerged from the data: (a) embracing diversity of d/Deafness, (b) passionate caring, (c) above and beyond expectations, (d) dedication to learning, and (e) beyond the classroom. There was one emerging theme, learning to collaborate, identified within research question one. There was no conflicting evidence that surfaced regarding how the program empowered and enabled teacher candidates to learn commitment to the deaf education profession. Following was the data that supported each theme.

Dominant themes of commitment. Embracing diversity of d/Deafness was the most prevalent of the dominate themes within MO-DESE's commitment to the profession pillar (MO-DESE, 2011, 2013). This embracing diversity of $d /$ Deafness theme was echoed from twelve (42.9\%) different participants. The next two dominant themes, passionate caring and above and beyond expectations, were heard from a total of 10 
(35.7\%) participants each. The final two dominant themes, dedication to learning and beyond the classroom, resonated with nine (32.1\%) participants per dominant theme. Following were the dominant themes that demonstrated how Midwest University empowered and enabled (Marquardt, 2011) teacher candidates to learn commitment to the deaf education profession.

Embracing diversity of d/Deafness. It was apparent across all participant groups that the program's espoused comprehensive philosophy transpired into practice (FF1, FF2, DEd2, DEd4, DEd7, 1TC1, OPP4, PCF2, STP1, STP2, STP6, 2TC1). As one student teaching preceptor articulated, "Once I got... [into] the field, it [mode of communication] [was] kind of an either/or thing" (STP1, p. 29, 590-591). She went on to explain that mode of communication should not be about "camp[s]" (STP1, p. 30, 614), but, about the individual; yet, she continued to feel pressured to pick a side. "What [mode of communication] works for one isn't always going to work for the next person" (STP1, p. 30, 617-618). She indicated that individuals from comprehensive programs tended to be very open to either approach.

The importance of choices in communication options was echoed by a deaf educator who said, “I don't feel like one type of 'deafness' or approach is always right and that, instead, it is a very personal decision based on what is best for each child and his or her family" (DEd4, p. 4). A per course faculty member agreed that it was about meeting each student's individualized needs and there was not just one way to go about doing that (PCF2). She said that meeting students' individualized needs could also include the use of Visual Phonics or Cued Speech. 
Diversity of $d /$ Deafness was seen in the responses of full-time program faculty as well. One faculty member said, "Too many times, we see candidates who consider themselves 'competent' teachers if they can sign. Teaching is much more than signing" (FF1, p. 2). This same faculty member also remarked that teacher candidates need to have "strong oral and written communication skills (sometimes sign language)" (FF1, p. 2). The other full-time faculty participant remarked that teacher candidates' involvement with the Deaf Community should not be at the exclusion of other groups of individuals who were d/DHH (FF2).

This diversity of $\mathrm{d} /$ Deafness theme was echoed through the voices of program students and the preceptors who had program teacher candidates during their student teaching experience. One way that student teaching preceptors noted the program's openness to oral and manual communication options was through the open-mindedness of the program teacher candidates (STP1, STP2, STP6). Preceptors also remarked on the teacher candidates' preparedness to meet the diverse learning needs of students who were $\mathrm{d} / \mathrm{DHH}$, including the auditory and visual needs of the learners (DEd2, OPP2, STP2).

One student teaching preceptor commented that both of the teacher candidates she had from this program were able to communicate based on the student's needs, whether their primary mode of communication was spoken English or ASL (STP2). Others concurred that the teacher candidates could meet the needs of students who communicated primarily in sign language or spoken language (STP1, STP4, STP6). Lastly, program students touted the program's unbiased approach regarding communication modalities. 
As one deaf educator stated, "This program did not force an opinion one way or another on its students" (DEd7, p. 4), instead the program focused on parent choice and students' needs. Another deaf educator phrased the program's openness to diversity of $\mathrm{d} /$ Deafness in this way:

I feel confident in the knowledge I acquired about hearing loss and deafness while in this program. It [the program] instilled in me a great respect for all people with hearing loss - whether they consider themselves deaf, Deaf, or hard of hearing. (DEd4, p. 4)

Passionate caring. The passionate caring dominant theme rang true across all general groups (FF1, DEd1, DEd2, DEd4, OPP2, OPP4, STP1, STP2, STP6, \& STP7). Additionally, passionate caring was confirmed in program documents (STED1A \& STED1B). Although MO-DESE's educator framework did not include anything specific to serving students who were $\mathrm{d} / \mathrm{DHH}$, the following student teaching preceptor exuded a determination to help all students who were $\mathrm{d} / \mathrm{DHH}$ when she said:

I am deaf myself... I'm just dedicated to helping... [students who are d/DHH] go farther in life because I know what I went through... these kids have a lot more potential and I just want to see them get there... in any way, shape, form, or fashion. (STP1, p. 81-86)

Her passionate caring embodied a determination to make a difference in the lives of students who were $\mathrm{d} / \mathrm{DHH}$. In addition, program professors were reported to demonstrate a passion for the field of deaf education (OPP4, STP2). One faculty member who was also a graduate of one of the most prestigious law schools in China demonstrated passionate caring when she said, "Deaf Education has always been in my heart...the 
world might not miss another Chinese lawyer, but an advocate serving the low-incident disability group is greatly needed" (FF1, p. 1).

A deaf educator described a person who shows commitment as, "someone [who] is willing to do something even when it gets difficult because they care about it" (DEd1, p. 2). She shared her interest, initially, within the broader field of communication disorders when she said, "Those who struggle to communicate...do not have the same access to opportunities and experiences" (DEd1, p. 1). Passionate caring, caring enough to make a difference with the students who were $\mathrm{d} / \mathrm{DHH}$ that they served or will serve, was evident at almost every turn.

One preceptor described the spark that ignited her passion in this way, "They [the students who were $\mathrm{d} / \mathrm{DHH}$ ] amazed me...they were so eager to learn...they just melted my heart!" (OPP2, p. 4. 58-60). A deaf educator stated, "There is nothing better than teaching a child a new concept and seeing their eyes and attitude spark with excitement and pride—-showing they have knowledge they didn't [have] before" (DEd4, p. 1). Another deaf educator said, “...this is absolutely what I am meant to do; every day I love coming to work" (DEd2, p. 1). Passionate caring was not limited to those who were already teachers.

The majority of the teacher candidates who completed student teaching during this three year period were reported to display a strong passion for the field (STP2, STP6, STP7). One student teaching preceptor who ranked passion for teaching as the number one commitment factor indicated, "passion was flowing" (STP7, p. 6, 96-97) from both Midwest University's teacher candidates. Another student teaching preceptor who also had two program teacher candidates described their passion in this way, "it's...that inside 
fire they [Midwest University teacher candidates] have to really motivate and get the kids to learn. And they just have this level of compassion and caring for the students" (STP2, p. $5 \& 6,106-108)$.

This passionate caring for the students they served was also evident in the student teaching evaluations (STED1 \& STED2). As one supervisor stated about a teacher candidate in a final student teaching evaluation, "She has sincere concern for her students and wants them to learn \& excel. She promotes her students strengths but is aware of their weaknesses" (STED1A).

Above and beyond expectations. Program faculty set the bar high (DEd1, OPP3, OPP4, 2TC1). The above and beyond expectations dominant theme emanated from the data (DEd1, FF1, FF2, OPP1, OPP3, OPP4, STP2, STP6, 1TC1, 2TC1). As one preceptor indicated, "the person who is really committed is go[ing] to go above and beyond" (OPP1, p. 14, 271). A student teaching preceptor concurred and indicated that the two teacher candidates she had, met that high mark (STP2). Both teacher candidates were "phenomenal" (STP2, p. 5, 98) examples of commitment. They had passion and a willingness to do whatever was needed to meet the individualized needs of the students. Another student teaching preceptor was impressed with Midwest University's teacher candidates' high level of commitment (STP6). She emphasized how the teacher candidates were there "before school, after school" (STP6, p. 8, 153). She indicated how one student used her before school time to organize so she executed a nearly seamless flow from lesson to lesson.

Teacher candidates were informed of program expectations at their graduate program orientation and throughout their program; time and effort were two of the 
expectations (FF1). One preceptor who was also a program graduate recognized the level of commitment that it takes to complete the program (OPP4). She indicated that teacher candidates who graduate from this program were most likely already demonstrating commitment to the field because of the program's high expectations and standards.

A deaf educator concurred (DEd1). She indicated that the program was challenging because of its content, but, also because of the high expectations of the professors. This deaf educator indicated the professors are continually providing honest feedback and, at times difficult to hear feedback, to encourage the ongoing growth and development of the teacher candidates (DEd1).

A practicum preceptor who had also served as a student teaching preceptor during this three-year period suggested that exposure to the real-world of teaching through practicum and student teaching experiences provided teacher candidates with hands-on experiences which helped to develop an "above and beyond" mindset (OPP1, p. 17, 347). The importance of practicum experiences in developing a high level of commitment was reiterated by a faculty member when she said, "The real experiences help them [teacher candidates] to see the importance of giving $110 \%$ to the people they serve" (FF2, p. 2). Interestingly, almost this exact sentiment was echoed by a 1st year teacher candidate when she indicated that it was important to give "one hundred and ten percent each day" (ITC1, p. 2) to demonstrate commitment to the profession. This high level of commitment was echoed in the teaching philosophy document received; there was a drive for giving the best education to all students (TPD1).

Dedication to learning. Teacher candidates were dedicated to learning (DEd2, DEd4, DEd6, DEd7, FF1, PCF2, STP7, 1TC4, 1TC5). There was an expectation by 
program faculty that teacher candidates needed to demonstrate a desire for learning (FF1). This was reflected in a student teacher preceptor's comment when she said that the teacher candidates she had from Midwest University were, "very willing to learn" (STP7, p. 27, 539). In addition, a deaf educator knew that she had a passion for learning, but, was not sure whether to credit the program for its development or whether it was a personal trait she possessed (DEd4).

Three primary elements were highlighted within this dedication to learning theme. The first element was that learning needed to be ongoing (DEd2, DEd6, PCF2). Next, learning could be achieved through a variety of avenues (DEd6, PCF2, DEd7, ITC5). And finally, the program's emphasis on ongoing learning was credited, in part, for teacher candidates' commitment to the profession (DEd2, DEd6, DEd7, ITC5, PCF2).

The program set the stage for an ongoing dedication to learning through resources. At times, the resources were directly provided by the program, for example providing a variety of sign language classes and providing ongoing learning opportunities through "numerous research projects" (DEd7, p. 2) that were a part of the program. The program also provided information on how to access resources beyond the program walls. For example, connections were made to people such as deaf educators in the field (PCF2), information was shared on upcoming speakers or workshops (ITC5), and teacher candidates were taught about how to find research in the field (DEd6).

Beyond the classroom. The dominant commitment theme which took teacher candidates beyond the classroom and into the community of individuals who are $\mathrm{d} / \mathrm{DHH}$ surfaced in a variety of ways. The beyond the classroom theme was provided through hands-on practicum experiences, a program staple and state and national CED 
requirement. One faculty member mentioned that although CED required 150 clock hours of practicum prior to student teaching that Midwest University required 150-200 contact hours (FF2). As one preceptor emphasized, "outside practicums are crucial...and the [on-site, program] preschool as well...that really helps you make the connection between what you're learning... and...how it's going to be used" (OPP1, p. 18, 358-361).

Program faculty encouraged and, at times, required teacher candidates to move beyond the practicum classroom and academic classroom into the Deaf Community (1TC6, OPP2, DEd4) and the greater community of individuals who are d/DHH (FF2, OPP2, 2TC1). This push to engage teacher candidates with individuals who were $\mathrm{d} / \mathrm{DHH}$ was mentioned as a positive program influence across all general invited participant groups for teacher candidates to learn commitment to the deaf education profession (1TC6, 2TC1, DEd4, FF2, OPP1, OPP2). As one preceptor indicated, the teacher candidates who get involved beyond the classroom demonstrated their commitment (OPP2).

Teacher candidates' learning was integrated with problem-based learning within coursework in the form of action learning projects designed to make a difference within the community (DEd6). Program teacher candidates explored solutions that addressed needs within the greater community that would impact individuals who were $\mathrm{d} / \mathrm{DHH}$. Play dates were arranged for young children who were $\mathrm{d} / \mathrm{DHH}$ which also integrated a “support group element for families" (DEd6, p. 2). Teacher candidates also created a communication book that included signs and a variety of strategies for improving communication with hospice patients with hearing loss. Finally, a classroom acoustics project was implemented at an off-site preschool (DEd6). 
Emerging themes of commitment. There was only one emerging theme that surfaced from the data within MO-DESE's commitment to the profession pillar (MODESE, 2011, 2013). The theme, learning to collaborate, was heard from six $(21.4 \%)$ participants. The learning to collaborate theme was heard from participants within all three general participant groups: (a) students, (b) faculty, and (c) preceptors.

Learning to collaborate. Learning to collaborate was the least prevalent of the commitment themes. Collaboration signaled one way in which the program enhanced teacher candidates' commitment to the deaf education profession (FF1, DEd3, DEd4, DEd6, OPP3, PCF2). One deaf educator mentioned the benefit of what she termed, "forced collaboration" (DEd4, p. 2) or group work with peers throughout the program.

"Forced collaboration" (DEd4, p. 2) was credited with "create[ing] a bond and a willingness to ask input from others when needed" (DEd4, p. 2). She further expanded that the bond created was indeed lasting. This deaf educator has continued contact with all of her peers that were in her cohort as well as other program teacher candidates, all three full-time program professors and the preceptors to whom she was assigned. In addition, she has continued to see her Deaf friends whose friendships formed while in the program.

Program faculty as well as a preceptor emphasized the importance of collaboration. There was an expectation of teacher candidates to exude a willingness to listen, learn, and collaborate with others (FF1). Another program faculty emphasized the importance of collaborative skills in order to be proficient in teaching students who are d/DHH (PCF2). Both a willingness to collaborate and the ability to carry out collaboration with professionals were deemed critical (PCF2). 
Although the collaborative aspect was not mentioned by the deaf educator who reported community projects that helped form teacher candidates' commitment, all three projects that were mentioned (DEd6) were collaborative projects. In addition, a preceptor recognized that team collaboration between the university supervisor and off-site practicum supervisor was instrumental for teacher candidate programming (OPP3). She also indicated that this collaborative team expanded to include the teacher candidate during the practicum experience.

Research question one, how the program empowered and enabled teacher candidates to learn commitment to the deaf education profession, yielded several compelling, dominant themes and one emerging theme. The data revealed that committed deaf educators from Midwest University (a) embrace diversity of d/Deafness, (b) care passionately, (c) exceed high expectations, (d) are dedicated to learning, and (e) are immersed beyond the classroom in experiences with individuals who are d/DHH. The emerging theme indicated that committed deaf educators need to learn to collaborate with others. There was no conflicting evidence that emerged from the data within the area of commitment to the deaf education profession. Embracing diversity of $\mathrm{d} / \mathrm{Deafness}$, the most prevalent of all the commitment themes, gave a resounding nod that Midwest University’s espoused comprehensive philosophy was indeed practiced.

\section{Research Question Two}

How does the program empower and enable teacher candidates to learn proficiency in practice with students who are d/DHH?

Proficiency in practice was the second of Missouri's educator pillars considered paramount to the development of quality teachers (MO-DESE, 2011, 2013). For this 
study, practice was defined as (a) providing meaningful learning opportunities adapted to diverse learners that support development of the whole student, (b) utilizing strategies that promote the development of students' critical thinking, problem solving, and effective communication skills, and (c) creating positive learning environments which promote student engagement, self-motivation, collaboration, and respect for all (MODESE, 2011). To determine the program's influence on teacher candidates' proficiency in practice, the researcher asked about the program's role in the development of teacher candidates' proficiency in practice.

In the area of proficiency in practice, the following six dominant themes emerged: (a) differentiated instruction, (b) how to teach, (c) preparedness, (d) flexibility, (e) building teaching competence through practice, and (f) knowledge through coursework. In addition, the data revealed three emerging themes (a) critical thinking, (b) evidencebased teaching, and (c) ongoing feedback loop. There was some conflicting evidence that surfaced regarding how the program empowered and enabled teacher candidates to learn proficiency in practice. The conflicting evidence revolved around understanding the public school system and having a stronger knowledge base for subject content areas. These deficit areas were further explored under research question four on program improvement. Following was the data that supported each theme.

Dominant themes of proficiency in practice. The most prevalent dominant theme within MO-DESE's proficiency in practice educator pillar (MO-DESE, 2011, 2013) was differentiated instruction. The differentiated instruction theme resonated with $12(49.2 \%)$ of the participants. The next most prevalent dominant theme was how to teach and it was heard from 11 (39.3\%) participants. The themes, preparedness and 
flexibility emerged from 10 (35.7\%) different participants each. The final two dominant themes, building teaching competence through practice and knowledge through coursework, were heard from eight (28.6\%) different participants per theme. Following are the dominant themes that demonstrated how Midwest University empowered and enabled (Marquardt, 2011) teacher candidates to learn proficiency in their teaching practice.

Differentiated Instruction. Differentiated instruction was the most prevalent dominant theme under research question two on proficiency in practice and it received equal billing to the embracing diversity of $\mathrm{d} /$ Deafness theme of research question one. Proficiency in teaching students who were $\mathrm{d} / \mathrm{DHH}$ was associated with meeting the diverse needs of this population of students whom they served (DEd2, DEd7, FF1, FF2, OPP1, OPP4, PCF2, STP1, STP2, STP4, STP6 1TC1). There was also confirming evidence within a student teaching evaluation document (STED1B).

Within the differentiated instruction theme, the importance of meeting diverse learners' mode(s) of communication needs was stressed (DEd2, PCF2, STP1, STP2, 1TC1). To be proficient in teaching, a teacher candidate needed to be able to teach in the student's mode of communication (FF2, PCF2, STP1, STP2). This differentiated instruction theme was additional evidence that this deaf education teacher preparation program's espoused philosophy was practiced.

Student teacher candidates were reported to demonstrate proficiency in teaching irrespective of the students' communication modality, manual or oral (STP1, STP2, STP6). A 1st year candidate recognized that being a strong signer was not enough (1TC1). Teacher candidates needed a larger skill set to meet the needs of this diverse 
population of students who were d/DHH. An off-site practicum preceptor mentioned that some of the teacher candidates she worked with had a strong knowledge base of cochlear implants and could apply both visual and auditory strategies to meet students who were d/DHH needs (OPP4).

There was much more to differentiated instruction than communicating in the student's primary mode of communication. Differentiated instruction may be best summed up by one student teaching preceptor's perception of a Midwest University teacher candidate when she said, "The student teacher was very attuned to what each student in the classroom needed...there was a wide variation of skill level...it required a lot of differentiation, and that was always, always present" (STP4, p. 8 \& 9, 166-169). Differentiated instruction started with planning for individualized needs within lessons (STP6) and then putting the plans into action (DEd7, STP4). Differentiated instruction also included adapting teaching materials to fit students who were d/DHH needs (STP2) and teaching at the student's language level (OPP1).

As one preceptor noted, "You have to meet them [students who are d/DHH] where they're at or you're not going to be able to teach them anything" (OPP1, p. 28, 576-577). This off-site practicum preceptor indicated that most teacher candidates from Midwest University were able to teach at students' language levels (OPP1). If a teacher candidate needed prompting in the area of teaching to the student's language level, the teacher candidate usually aimed too high (OPP1). There was confirming evidence of this differentiated instruction theme within a final student teaching evaluation. The university supervisor remarked how the student teacher displayed strong critical thinking 
skills that were reflected in her awareness and insights into students' individualized needs (STED1B).

How to teach. As the researcher combed through the data, how to teach was not the first dominant theme to surface, nor was it the second. The themes differentiated instruction and preparedness were the dominant themes that surfaced first. However, the 'how' of teaching was the reality that resonated in each of the dominant and emerging themes within this educator pillar (DEd4, DEd5, DEd7, FF1, FF2, OPP1, PCF2, STP6, 1TC4, 1TC6, 2TC1). Therefore, much of the how to teach theme came to light within the other dominant and emerging themes.

Program faculty who had taught students who were d/DHH for 15-17 years each zeroed in on the nuts and bolts of teaching (FF2, PCF2). Goal-directed lesson planning, differentiated instruction with a focus on effective communication across modalities, flexibility, and classroom and behavior management resonated within their responses. Lesson planning was a process which included several key components from planning (FF2, PCF2) and implementation (FF2, PCF2) to evaluation (FF2). And teacher candidates had to know a variety of teaching strategies, including methodologies for teaching students who were d/DHH (FF2). Program students concurred (DEd5, 1TC5, 1TC6).

Teacher candidates acknowledged that they were taught effective ways on how to teach students who were d/DHH (DEd5, 1TC5, 1TC6) using different methods and strategies (DEd5, 1TC4) such as direct teaching of concepts (1TC5) and infusing teaching with enhanced visuals (1TC4). An off-site preceptor agreed that most practicum students knew "how to teach" (OPP1, p. 2). Lesson plans were pivotal to effective 
teaching and there were ongoing opportunities within practicum experiences to practice lesson plan writing and implementation of lesson plans (FF2, DEd4, 1TC1, PCF2). Lesson plan writing and implementation seemingly connected to the positive outcomes. A former program student stated, "I think the consistency in expectations across practicum courses resulted in my continued improvement and growth in writing and executing lesson plans” (DEd4, p. 2). In addition, student teaching preceptors found Midwest University teacher candidates to have strong lesson plan writing skills (STP2, STP4, STP6, STP7). As one student teaching preceptor stated:

It's like they [student teacher candidates] get to the meat of what needs to be taught...their lessons are focused, and are meaningful. They're not just a bunch of fluff because they've taken the time to analyze what the goals are and what the curriculum is, which is what was hit so hard in their preparation program. (STP2, p. 11, 218-224)

And skilled teacher candidates were noted to teach across the curriculum as well. As another student teaching preceptor remarked, "I think my last student teacher [from Midwest University] was the best [at teaching across the curriculum]...I was just amazed at how she went from 8:00 to 3:00 and just integrated everything in every part of her day. There was confirming data on teaching across the curriculum within final student teaching evaluations on one teacher candidate (STED1A, STED1B). Both the student teaching preceptor and university supervisor noted how well the student taught concepts across the curriculum. This same student teacher was also encouraged to expand her teaching of concepts across multiple days (STED1B). 
Preparedness. Preparedness was another dominant theme that emerged from the data to answer question two on proficiency in practice. The preparedness theme was heard from the voices of a variety of participants (FF1, FF2, DEd6, OPP1, OPP4, STP2, STP4, STP6, STP7, 2TC1). And as one preceptor stated, "There's a lot to Deaf ed... we're K-12, we're all seven subjects" (OPP3, p. 16, 296-297). With deaf education covering a broad spectrum, the preparedness of teacher candidates was of paramount importance.

At Midwest University, teacher candidates were immersed in both learning (FF1, FF2, OPP1, STP7) and practice during graduate school (FF2, OPP1, STP7). Both book knowledge and experiences were deemed important to becoming proficient in teaching (FF2, OPP1, STP7). One preceptor acknowledged that the program had "a nice balance... [between] book knowledge and the life experiences" (OPP1, p. 19, 193-194).

The program was also reported to provide resources to teacher candidates to assist them in becoming proficient in their teaching (OPP4, STP2, 2TC1). Teacher candidates were reported to arrive at their student teaching placements with an abundance of resources from Midwest University (STP2). Some of the resources were new to the preceptor and were then added to her repertoire (STP2). One deaf educator mentioned how the resources that teacher candidates' created during class projects not only addressed community needs, but, would be beneficial to the teacher candidates in the future (DEd6).

There were a few powerful quotes that emerged from the data about how well prepared the teacher candidates from Midwest University were for student teaching (STP2, STP4, STP6, STP7). Perhaps the most compelling evidence for the preparedness 
theme was that the quotes selected for use came from the three preceptors who were not program graduates of Midwest University (STP4, STP6, STP7). The first preceptor mentioned below also commented on her own program preparation.

"Everybody that we have seen from your program [Midwest University] is top notch" (STP4, p. 13, 250-251) stated one student teaching preceptor. She went on to say, “They [Midwest University teacher candidates] come in and they're confident...they're prepared, they're ready to jump in and grab the bull by the horns and I did not feel that way coming out of my program" (STP4, p. 13, 257-259).

Another preceptor who was not a program graduate and who had two student teachers indicated that the teacher candidates did not just have book knowledge, "they [knew] how to use it" (STP7, p. 8, 150). The next student teaching preceptor also had two student teachers from Midwest University. Although she commented on one of the two student teachers getting off to a rockier start which she attributed to nerves, she stated, "The two student teachers that I've had [from Midwest University] have been more prepared than any other student teacher I had from other programs” (STP6, p. 9, 172-173). This same preceptor, who was also not a program graduate, did not see any program adjustments that were needed to enhance proficiency in practice of the teacher candidates. A deaf educator agreed that she was prepared for teaching when she said, "I feel like my training was very beneficial to the job I currently hold” (DEd6, p, 4).

Flexibility. Flexibility, another dominant theme in the area of proficiency in practice was believed to be an important skill across all general participant groups (DEd3, DEd6, FF2, OPP2, PCF2, STP4, STP6, STP7, 1TC4). It was touted by one preceptor as the number one thing a teacher candidate needed in order to be proficient in teaching 
(STP7). This student teaching preceptor believed that Midwest University teacher candidates stood out from the crowd in the area of flexibility. She said, "I get student teachers from two different programs...there's a lot of them that are not trained to be flexible...the [teacher candidates] I've had from [Midwest University] are very flexible" (STP7, p. 9, 166-172).

An off-site preceptor agreed that flexibility was critical. She said, "flexibility is a huge one [characteristic needed by teacher candidates]" (OPP1, p. 7, 145). According to the participants, flexibility fits a variety of areas. Flexibility was seen in openmindedness to how students' learn (STP4) including adjusting to the students' mode of communication (1TC4). Flexibility also included a willingness to seek out information "to try new things" (STP4, p. 6, 109-110), as well as having the ability to monitor and adjust, (STP4, STP6). One preceptor described a Midwest University teacher candidate on the subject of flexibility in this way, "She was flexible, monitoring and adjusted [based on] how each student is different... your standard classroom behavior management might be one way; you might have to deal with another student another way" (STP6, p. 11, 220-223).

Another preceptor described this aforementioned flexibility as a necessary skill that allowed teacher candidates to "think on their feet" (OPP2, p. 13, 251). This type of on-the-spot flexibility required practice (DEd6, 1TC4, OPP2) and a skill that some offsite practicum students do well with and others still require more practice (OPP2). The on-site preschool was one place that flexibility was learned (DEd6, 1TC4). As one deaf educator said: 
At [Midwest University] we received many opportunities to work on flexibility...[were] given reminders and advice, but also allowed to figure it out on our own. It may have taken me awhile to get it right, but it was a valuable skill to gain and I credit my experiences in the Deaf Preschool for preparing me in that way (DEd6, p. 3).

Building teaching competence through practice. On-site and off-site practicum experiences were deemed critical for developing proficiency in teaching among participants (DEd1, DEd4, DEd6, OPP1, OPP2, PCF2, STP2, 1TC1). As one student teaching preceptor said, "Getting [teacher candidates] outside of their classroom and in other sites practicing... skills before they come to student teaching is $100 \%$ beneficial" (STP2, 187-188). Another preceptor said, "You're learning it and you're using it, basically at the same time and that's really what you need to really become a proficient teacher, because you need that, you need the balance of both [book knowledge and practice teaching] (OPP1, p. 20, 413-415).

This deaf education teacher preparation practicum experiences are sequential (FF2, DEd1, OPP2, STP2) beginning with observations, seeing other teachers teach (DEd4, DEd6, OPP1, OPP3, STP2) and experiences in the preschool for young children who are d/DHH (FF2, OPP2, STP2). The on-site preschool program was credited as a great hands-on learning opportunity (DEd6, OPP1). A deaf educator said that the preschool environment played an important role in building behavior and classroom management skills as well as flexibility in teaching (DEd6).

Off-site preceptors noted advantages to the on-site preschool as well (OPP1, OPP3). The preschool environment provided an opportunity to learn and use SMART 
Board technology (OPP3). Another off-site practicum preceptor was quoted saying, "There's a high standard expected of the teachers [teacher candidates] as far as what they're learning and what they're expected to perform...especially with the preschool practicum" (OPP1, p. 21, 430-433).

The preschool teacher and program faculty knew what teacher candidates were learning and expected to see the learning in the their practice (OPP1). This preceptor who was also a program graduate believed this extra knowledge advantage may not be as easily replicated at off-site practicum sites. "They [off-site practicum preceptors] may not be holding you quite as accountable for some...things" (OPP1, p. 22, 441-442) because these preceptors were not as familiar with what teacher candidates were learning.

The aforementioned off-site preceptor believed it was important to have both onsite and off-site practicum experiences; teacher candidates needed to have different perspectives (OPP1) and experiences (DEd6, STP2). The sequential layout of practicum provided a linkage of skills to build a strong skill set. While practicum opportunities provided some exposure to hearing technology, student teaching provided more opportunities for refinement of skills, for example, with troubleshooting assistive listening devices (STP2). In another example, practicum experiences typically occurred in three consecutive hour sessions, one day a week. During student teaching, teacher candidates were required to be at school when their student teaching preceptor was present. "They're not just here from 9-4 when the students are here" (STP2, p. 18, 364) which gave the teacher candidates "the true experience of what it's like to be a teacher" (STP2, p. 18, 377-367). 
Knowledge through coursework. Coursework knowledge was deemed critical for the development of proficiency in practice (FF1, OPP1, 1TC1) and knowledge in the area of d/Deafness and hearing loss was recognized as a notable strength (DEd1, DEd4, DEd6, STP1, STP2). The aforementioned eight participants put knowledge within the dominant theme category for proficiency in practice.

As one preceptor commented, coursework provided teacher candidates with "a depth and breadth of knowledge in our field" (STP2, p. 6, 120-121). A deaf educator indicated that her knowledge within the field of deafness came through Midwest University when she said, "I do not have a hearing loss and knew very little about deafness and hearing loss prior to this program" (DEd5, p. 4). Research was also an important component for building knowledge during the program (DEd4, DEd6, DEd7) and beyond (DEd5, 1TC4).

Research was integrated within coursework. As one deaf educator remarked, "we researched the population of DHH students that have the highest rate of success regarding reading comprehension and writing. We explored methods used with hearing students and accommodations that can be used to transfer successful methods to students with a hearing loss" (DEd6, p. 4). The program was also noticed for the development of knowledge in the areas of speech (STP1, STP2) and auditory and language (STP1).

Emerging themes of proficiency in practice. There were three emerging themes that surfaced from the data within MO-DESE's proficiency in practice pillar (MO-DESE, 2011, 2013). The themes were: (a) critical thinking, (b) evidence-based teaching, and (c) ongoing feedback loop. The first emerging theme, critical thinking, was heard from six 
(21.4\%) of the participants. The latter two themes were heard from five (17.9\%) different participants each.

Critical thinking. The critical thinking theme was echoed from some participants (DEd2, DEd4, FF2, OPP2, STP2, 1TC1). Teacher candidates were expected to critically reflect on and evaluate their teaching (DEd2, DEd4, OPP2, STP2, 1TC1). Critical thinking included reflecting on what went well within a lesson and what could be changed to better address students' needs (DEd2, DEd4, FF2). Critical reflection also included thinking ahead in the lesson planning process. Teacher candidates were expected to anticipate potential challenges and to think through potential solutions (FF2, 1TC1).

Reflection on teaching was also viewed as a way to develop ownership of one's teaching. One student teaching preceptor acknowledged the importance of the program's focus on the teacher candidates' requirement of lesson reflection, through journaling and other avenues, and its role in the teaching process (STP2). This student teaching preceptor believed it was, "very beneficial to have that reflective piece... as teachers... we [need to] take ownership... [asking] what can we do next time...[Reflection on practice] needs to just become a habit and something we do more naturally (STP2, p. 17, 343-353).

Evidence-based teaching. The evidence-based teaching emerging theme also known as data-driven teaching was heard from a few participants (DEd2, DEd4, DEd5, DEd6, FF1). One deaf educator noted that evidence-based teaching that she learned in the program was pivotal for her career (DEd2). Another deaf educator mentioned that the program highlighted different ways to assess student learning (DEd5). Still yet a 
preceptor noted that there was a distinct and direct program emphasis on linking lessons to the student's IEP and the curriculum (STP2). The aforementioned data paralleled with a faculty member who mentioned the program's focus on connecting assessment to teaching; teaching needs to be based on evidence (FF1).

Ongoing feedback loop. Feedback was the final emerging theme under proficiency in practice and it was noted by a few participants (DEd1, DEd4, FF2, STP2, STP7). Teacher candidates were provided with ongoing feedback throughout their practicum and student teaching experiences (DEd1, DEd4, FF2). One faculty member stated that "teacher candidates are provided with a massive amount of written qualitative and quantitative feedback" (FF2, p. 3) to support them in improving their teaching practice. Improved teaching and continual growth as a result of professors' feedback was noted by deaf educators (DEd1, DEd4, DEd6). In addition, teacher candidates were expected to make adjustments based on feedback and did so (STP2, STP7). As one preceptor indicated, "if they [teacher candidates] needed help, they took the help... worked on it and improved" (STP7, p. 18, 360-362). This responsiveness to feedback was confirmed in a student teaching evaluation when a supervisor noted the strong student teacher follow through with recommendations made at midterm (STED2A).

More themes emerged from research question two, how the program empowered and enabled teacher candidates to learn proficiency in practice than any other research question. The dominant themes revealed that deaf educators from Midwest University develop proficiency in their teaching practice through coursework and experiences. Specifically, the deaf educators (a) learn to differentiate instruction to meet diverse learners' needs, (b) learn the nuts and bolts of how to teach, in which the lesson planning 
process plays a critical role, (c) become highly prepared, (d) develop flexibility to make on-the-spot adjustments, (e) build teaching competence through practice, and (f) deepen their knowledge through coursework. Differentiated instruction, the most prevalent of all proficiency in practice themes, provided additional confirmation that Midwest University's espoused comprehensive philosophy was practiced. There was some conflicting evidence that emerged from the data within the area knowledge, specifically knowledge in navigating the public school system and subject content knowledge and methodologies. The emerging themes indicated that proficient deaf educators need to (a) develop strong critical thinking skills, (b) implement evidence-based teaching, and (c) engage in an ongoing feedback loop for continued growth.

\section{Research Question Three}

How does the program empower and enable teacher candidates to create learning impact in students who are $d / D H H ?$

Learning impact was the third of MO-DESE's educator pillars considered paramount for quality teachers (MO-DESE, 2011, 2013). This pillar addressed the actual learning impact on the students whom they served. For the purposes of this study, learning impact was planned to be accomplished through the perceptions of the participants as well as through archival program clinic documents. The 26 completed Preschool Evaluation of Services documents (PED1-PED26) were aimed to gather evaluative data on the services provided to the preschoolers who were $\mathrm{d} / \mathrm{DHH}$ enrolled in the program. However, due to the high probability of skewed results as discussed previously, PED1-PED26 documents were, instead, used to confirm or disconfirm data. 
In the area of learning impact, there were no dominant themes that emerged from the data. There were four emerging themes that surfaced: (a) meeting diverse learners' needs, (b) collaborating with educators, (c) teaching beyond academics, and (d) connecting with and engaging students. There was no real conflicting evidence that surfaced specifically in the area of learning impact.

Dominant themes of learning impact. There were no dominant themes directly evident within research question three. However, meeting diverse learners' needs was directly linked with the differentiated instruction theme within research question two on proficiency in practice. Therefore, the researcher only pulled out specific examples of meeting diverse learners' needs that were not used to support the differentiated instruction themes of research question two. In other words, the researcher did not use the same evidence in both proficiency in practice and learning impact.

Emerging themes of learning impact. There were four emerging themes that surfaced from the data within MO-DESE's learning impact pillar (MO-DESE, 2011, 2013). Midwest University teacher candidates were working towards: (a) meeting diverse learners' needs, (b) collaborating with educators, (c) teaching beyond academics, and (d) connecting with and engaging students. The voices of six (21.4\%) participants each were heard within the first three emerging themes which made them the most prevalent themes within learning impact. The final emerging theme, connecting with and engaging students was heard from five (17.9\%) different participants. The Preschool Evaluation of Services archival documents yielded confirming evidence within all emerging themes. 
Meeting diverse learners' needs. Meeting diverse learners' needs was an emerging theme within the area of learning impact. Some participants shared evidence on ways in which the teacher candidates affected the learning outcomes in the students who were d/DHH that they served (DEd6, OPP1, OPP2, OPP3, STP1, STP6). Some research participants cited specific examples of student learning (DEd6, OPP1, STP1, STP6). There was limited consistency within the examples, which more clearly articulated that teacher candidates addressed the individualized and varied needs of the students who were d/DHH whom they taught.

One deaf educator indicated that her knowledge of hearing loss and her ability to relate that information in lay terms helped her "to convince my [her] district to buy FM systems for classrooms" (DEd6, p. 5). She also adapted information learned within the program on assessment and identification of error patterns in reading to meet a particular student's need. This deaf educator reported, "Although this method was not directly taught... my ideas were formed directly as a result of the information I learned" (DEd6, p. 4). In another example, a student teaching preceptor noted, "Although Visual Phonics wasn't used in this school, it was good to see the teacher candidate use it and to witness gains with some of the students with profound hearing loss" (STP6, p. 13, 246-247).

Meeting diverse learners' needs was also confirmed through the Preschool Evaluation of Services documents. The number one reported learning and improvementfocused comments centered on communication (PED3, PED6, PED7, PED11, PED19, PED21, PED24). A couple of parents only mentioned speech development, specifically how their child improved in speech (PED6, PED11) and a couple of others mentioned speech therapy services were key within the program (PED7, PED19). Still other family 
participants mentioned manual communication options or a combination of both manual and oral methods (PED21, PED24). There was no way to know if each pair of the aforementioned comments was made by the same or different families.

One parent was pleased with, "how far our child has come in speech with your program" (PED, 11, p. 2). Another parent mentioned other family members' comments "about how good her speech has become" (PED6). Another parent said, "My child is gaining more ways to speak and sign" (PED21). Still yet another family participant mentioned how far her child's "speech and signing" had developed "since we started until now" (PED24, p. 2). That child had been attending the preschool program for over one year. Visual Phonics learning was also indicated as important learning through the on-site preschool program (PED3).

Collaborating with educators. Collaborating with educators was another emerging theme in the area of learning impact. Collaborating in off-site placements provided benefits to the teacher candidates as well as the preceptors. Some preceptors mentioned that they had learned from the teacher candidates that were assigned to their school (OPP1, OPP2, STP2, STP4, STP6, STP7). Teacher candidates walked away with new resources (STP2), new lessons (STP6), new materials (OPP1, STP7) and new ideas (OPP2). One preceptor mentioned, "I...learn[ed] a lot from the [student] teacher in my room" (STP4, p. 7, 129).

Preschool family participants noted the significance of their learning within the archival documents. One parent reported, "Our entire family has learned a lot" (PED1, p. 2). Another parent reported how their family had benefited from the information and resources received from the program's preschool (PED7). 
Teaching beyond academics. Teaching beyond academics surfaced within some of the participants' responses (DEd7, OPP1, OPP2, PCF3, STP1, 2TC1) in the area of learning impact. These participants made it clear that teachers of students who were $\mathrm{d} / \mathrm{DHH}$ had responsibilities in teaching beyond book knowledge. One faculty member mentioned that teaching students how to use an interpreter effectively also meant how "not to have students become dependent on them [interpreters]" (PCF3, p. 2). Having connections with other individuals who were d/DHH was stressed (PCF2, STP1) as well as fitting in socially in a hearing world (OPP1, OPP2, STP1, 2TC1). The following powerful quote by an off-site preceptor who was hearing summed up its importance: If we're expecting these kids to go into that mainstream classroom, you need to know if their maturity level or their vocabulary, or the things they are talking about don't match up at all with their peers...[that will] make them stand out even more from what I've seen. If they don't know who Taylor Swift is and everybody else in their classroom does, that might be something you talk about one day so they have that bit of information too. (OPP2, p. 19, 362-368).

The importance of socialization was also reiterated by preschool family participants (PED18, PED 20, PED22). At this early preschool stage, one parent reported, my child "has learned to interact with children" (PED18, p. 1). She believed this was one of the most important positive aspects of the program. Another parent agreed (PED20). Still yet, another parent indicated the importance of interacting with the teacher was "the best part of the program... She provides a positive learning environment and has the knowledge to help them meet and exceed their potential" (PED22, p.2). 
Connecting with and engaging students. The connecting with and engaging students' theme was the final and least prevalent of the emerging themes under research question three on learning impact. It was heard by a few participants (DEd4, OPP1, OPP3, STP1, STP2). An off-site preceptor indicated that that her students wanted to come back for more. The teacher candidate "got to know the students" (OPP3, p. 17, 315 ) and used that to her advantage. She "adapted SMART Board lessons and got the kids actively involved" (OPP3, p. 17, 321). This teacher candidate's lessons were motivating; the lessons were "enjoyable [and] interesting, but they worked on a skill" (OPP3, p. 17, 318). The students were not even aware they were learning.

The importance of connecting with the students and preparing and implementing engaging lessons has been noted within the data (FF2, STP1, STP2). A student teaching preceptor shared an example of a student who was struggling with mathematical concepts in the area of graphing (STP1). The teacher candidate used the child's interests and prior knowledge as a springboard for building the new concepts. This preceptor mentioned that the child's general education teacher reported that the student had mastered the mathematic concepts which had been a struggle for the student. The mastery of the graphing skills occurred after the teacher candidate had targeted the aforementioned lesson(s) using the student's interest and prior knowledge. The student teaching preceptor also mentioned that building from what the student already knows increases the student's confidence (STP1).

The connecting with and engaging students' theme was also echoed from preschool family participants (PED1, PED5, PED10, PED12, PED17, PED17, PED18, PED24, PED25). As with the aforementioned examples, preschoolers wanted to come 
back for more; there was a connection. School was talked about at home. One parent reported, "[my child] talks about her [the teacher] all the time at home" (PED1, p. 2). There was a love of school (PED10, PED12, PED24, PED25) and a love for the teacher(s) within the classroom (PED1, PED18, PED25).

Research question three, how the program empowered and enabled teacher candidates to create learning impact in the students who were $\mathrm{d} / \mathrm{DHH}$ they serve, yielded no dominant themes and four emerging theme. Although there was no dominant theme reported, there was significant overlap between the most prevalent proficiency in practice theme, differentiated instruction, and one of the top emerging themes in learning impact, meeting diverse learners' needs. The researcher attempted to separate proficiency in practice from learning impact; however, these two MO-DESE pillars (MO-DESE, 2011, 2013) were closely interconnected. In fact, it seemed somewhat unfounded to try to distinguish differentiated instruction from meeting diverse learners' needs.

The emerging themes indicated that deaf educators needed to create learning impact in students who were d/DHH by: (a) meeting learners' diverse needs, (b) collaborating with other educators, (c) teaching beyond academics, and (d) connecting with and engaging the students. There was evidence that all four of these emerging themes resonated through the program's archival documents. In total, the emerging themes resonated through $16(61.5 \%)$ of the 26 archival preschool documents. There was no conflicting evidence that emerged from the data within the area of creating learning impact. 


\section{Research Question Four}

How could the program improve to empower and enable the learning of teacher candidates who are prepared to teach students who are d/DHH?

MO-DESE's educational framework was designed to develop quality teachers who affect the continual growth and improvement of all students they teach (MO-DESE, 2011, 2013). So too Marquardt espoused that learning organizations must grow and learn to thrive (Marquardt, 2011). The researcher sought to uncover the ways in which Midwest University's deaf education teacher preparation program could improve. Specifically, the research question asked how the program could improve to empower and enable the learning of teacher candidates who were prepared to teach students who were $\mathrm{d} / \mathrm{DHH}$.

For this research question, the researcher believed that the most important participant groups for uncovering the ways in which the program could improve were two sub-groups of external stakeholders (a) the seven deaf educators (DEd1-DEd7), and (b) the five student teaching preceptors (STP1, STP2, STP4, STP6, STP7). The seven deaf educators were recent program graduates and in their first or second year of realworld teaching of students who are $\mathrm{d} / \mathrm{DHH}$ at the time of this study. Since they were recent program teacher candidates and were at the beginning of their teaching careers, they should have a clear perspective on what they had learned through the program and the gaps that existed.

The other external stakeholder group mentioned above was the five student teaching preceptors. They had the most direct knowledge of the teacher candidates' performance in teaching students who were $\mathrm{d} / \mathrm{DHH}$ in the teacher candidates' final 
semester in the program. In addition, this pool of five student teaching preceptors served students who were deaf or hard of hearing in a variety of settings (a) public school districts, and (b) a School for the Deaf. They also varied in their type of teaching position (a) an itinerant, rural co-op position primarily serving one student at a time across multiple grade levels and school districts, (b) some variation of a resourcemainstreamed classrooms serving students who are d/DHH at one grade level up to eight grade levels, and (c) a self-contained classroom at a School for the Deaf.

It was important that some of the preceptors were not program graduates because their voices could add a unique perspective. Three $(60 \%)$ of the five student teaching preceptors were not graduates of Midwest University. The remaining two (40\%) student teaching preceptors graduated from this deaf education teacher preparation program. It was also believed that another external stakeholder group, the four off-site preceptors (OPP1-OPP4), would add valuable input on ways the program could improve to better support the preparation of students at the mid-point of their practice experiences. They could provide a unique perspective to the level of preparation following the first year of on-site preschool practicum.

There was one dominant theme and one emerging theme that rose to the top from the data within the area of program improvement. Some areas of improvement were not stated when asked specifically about how the program could improve, but instead embedded within other content responses. The researcher looked for negative cases regarding the empowered and enabled learning of teacher candidates throughout the data to report on areas for program improvement. 
Dominant themes of program improvement. There was only one dominant theme that surfaced from the data within the area of program improvement. The theme, public school 101, was heard from eight (28.6\%) of the participants. Almost all of the deaf educators, six out of seven $(87.5 \%)$ believed they needed more knowledge and, at times, experiences within public schools.

Public school 101. Seveal participants, the majority of which were deaf educators, believed that teacher candidates needed more knowledge and/or experience to navigate life within the public school system (DEd1, DEd 2, DEd4, DEd5, DEd7, OPP, OPP1). One participant stated, "The program could have provided more information or opportunities to learn about the everyday workings of a public school” (DEd7, p. 3). While that deaf educator wanted to understand the interworking of the educational system better, most participants identified specific needs (DEd1, DEd2, DEd4 DEd5, DEd6, DEd7, OPP1, OPP2).

Three participants believed it was important for teacher candidates to have knowledge about the Common Core (DEd2, DEd5, OPP2). Two participants believed it was crucial that teacher candidates were more prepared to work with interpreters (DEd4, OPP1). As one preceptor said, "it's so crucial... you get along with your [student's] interpreter" (OPP1, p. 40, 826-827); but, she recognized that sometimes challenges were experienced by herself and other deaf educators. She indicated that interpreters "can give you [the deaf educator] such an important perspective" (OPP1, p. 41, 838-839) but it requires teacher candidates' preparation in developing interpreter expectations.

One deaf educator thought understanding the itinerant model of teaching students who were $\mathrm{d} / \mathrm{DHH}$ was so important that it justified a whole class devoted to learning 
about itinerant teaching challenges and discovering solutions (DEd4). One participant said, "I would've liked more information/practice with the SPED [special education] process" (DEd1, p. 3). Another participant wanted more experience in the SPED process, specifically with writing IEPs (DEd4). The final suggestion was that teacher candidates should have some experience with students who are d/DHH across all educational levels (a) early childhood, elementary, junior high, and high school (DEd6). This participant also recognized that it would be difficult for the program to ascertain those varied grade levels for every program teacher candidate (DEd6).

Emerging themes of program improvement. There was only one emerging theme that surfaced within the area of program improvement. The emerging theme was heard from six (21.4\%) of the participants. As with the dominant theme, the majority of participants who voiced this theme were deaf educators. Four (57.1\%) of the deaf educators believed that they needed more subject content knowledge and methodologies.

Subject content and teaching methodologies. The need for better preparation in subject areas was mentioned by mostly deaf educators (DEd2, DEd4, DEd6, DEd7) and a couple of preceptors (OPP1, STP1). Math (DEd2 \& DEd6), science (DEd2 \& DEd6), and reading (DEd2 \& STP1) were the most frequently mentioned subjects. History was also mentioned as a subject content area of need by one deaf educator (DEd2).

Subject areas such as math, specifically upper level math (DEd6), science, and history were pretty clear cut. According to the aforementioned deaf educators, the program did not seem to provide the teacher candidates with enough knowledge or experiences to feel competent in teaching those content areas. The area of reading, however, revealed some inconsistencies. 
As previously mentioned, one deaf educator indicated how she was applied her reading knowledge learned within the program and successfully adapted it to meet a learner's reading needs (DEd6). She also mentioned how the program emphasized current research and practices within the area of reading. Yet another deaf educator believed, "we [teacher candidates] could have been more prepared to teach a variety of reading programs" (DEd2, p. 3). She further added that if a district was not sold on a particular program, "it is difficult to find a program that works for the student when you do not have a good base to pull from" (DEd2, p. 3). Additionally, one student teaching preceptor was uncertain if one of the student teacher candidate's that she had from the program had simply made a mistake in designing an appropriate reading lesson or if the student did not understand reading development (STP1).

There was a need expressed for content knowledge as well as methodologies for teaching the specific content (DEd2, DEd6). One deaf educator credited her student teaching experience as being the place that she learned the most about teaching methodologies (DEd2). Additionally, teacher candidates needed to understand subject content as it related to development. As one student teaching preceptor mentioned it was important for teacher candidates to be able to look at a general IEP goal and have enough background knowledge to know what the students needed to learn in order to reach that ultimate goal (STP1). This need for increased knowledge was confirmed within a student teaching final evaluation when a preceptor stated that the student teacher needed more knowledge of the "developmental timeline for specific academic skills" (STED1A, p. 5). Research question four, how the program could improve to empower and enable the learning of teacher candidates who are prepared to teach students who are $\mathrm{d} / \mathrm{DHH}$ 
resulted in one dominant and one emerging theme. The dominant theme, public school 101, indicated that Midwest University should better prepare their teacher candidates to understand and navigate the public school system. The emerging theme, subject content knowledge and methodologies, indicated that Midwest University should increase the teacher candidates learning in specific subject content areas and teaching methodologies.

\section{Additional Findings}

There were three topic areas that, from the researcher's perspective, appeared to be potential areas of omission or disconnect within the findings. The first topic area was classroom and behavior management. It was difficult for the researcher to imagine a deaf educator being proficient in practice and creating learning impact without strong classroom and behavior management skills. The area of classroom and behavior management, however, revealed inconsistent and sporadic results within the findings. The second topic delved into the teacher candidates' heavy workload requirements within this deaf education teacher preparation program. This topic also yielded inconsistent findings.

The final topic area was distinctly different from the aforementioned topic areas. This distinct area seemed more subtle, yet, pulled at the researcher in a way that the researcher was compelled to include it within the additional findings section. The final area was connected to the teaching beyond the classroom theme which, in part, emerged to answer research question three. The focus of this topic area was the differences that surfaced between responses from participants who were d/DHH and those participants who were hearing.

The role of classroom and behavior management in teaching. Although, classroom and behavior management did not emerge as a theme, its significance was 
heard from particular participants. There were two program faculty, the faculty with the most years of teaching experience with students who were $\mathrm{d} / \mathrm{DHH}$, who mentioned the importance of managing a classroom and students' behavior. Both faculty commented on the importance of classroom and behavior management within the area of proficiency in practice. As stated by one faculty, proficient teacher candidates needed to be able to implement "appropriate preventative strategies and behavior management in a group of learners" (FF2, p. 2). The other faculty member stressed the importance of "impeccable classroom discipline" (PC2, p. 2) to display proficiency in practice.

Beyond the aforementioned faculty, classroom and behavior management was mentioned sporadically among the other groups of participants. And what was heard differed significantly among some participants. Participants seemed to mention classroom and behavior management if it was either a significant area of need for continued growth (DEd5, OPP1) or a significant strength (STP6) or implied strength (OPP3) among the teacher candidates.

One deaf educator remarked that the on-site preschool program was a great environment in which to learn behavior management skills (DEd6). Yet, following a year of practicum within the on-site preschool program, an off-site practicum preceptor reported, "most of them [teacher candidates] struggled... with problem behaviors" (OPP1, p. 24, 479-480). Conversely, another off-site preceptor implied that the teacher candidate had strong behavior and classroom management skills when she remarked on the engaging lessons that were taught by the teacher candidate that had her students wanting to come back for more (OPP3). 
Within the realm of student teaching, one student teaching preceptor reported, "we practice Love and Logic [to manage classroom behaviors]... she [a program student teacher] was very, very good at that" (STP6, p. 12, 225-227). However, a deaf educator reported that her biggest challenge within the classroom, post-program graduation, was behavior management and school to home carryover (DEd5). As a result of this study, the area of classroom and behavior management may warrant further exploration to understand the influence of Midwest University in empowering and enabling teacher candidates who are ready to teach students who are d/DHH.

Teacher candidates' workload. The program's 'above and beyond expectations' and 'beyond the classroom' experiences were two of the dominant themes that rose to the surface within research question one. While these themes were strongly supported positive themes for the teacher candidates' commitment to the deaf education profession, the researcher also noticed comments regarding teacher candidates' workload. Most comments were general, consistent comments about the program being tough and a lot of work (DEd1, DEd4, OPP1, 1TC1). As one 1st year teacher candidate stated, "I am only halfway through the program and although it is tough work I don't regret a single minute of it. I am learning so much... it is all valuable... I know I need all of it to become an excellent teacher" (1TC1, p. 3).

Other comments surfaced specific to workload that centered more on paperwork related to lesson plan writing (DED4, OPP1, STP7, 1TC1). Yet what was heard from the participants, varied significantly. Another aspect suggested by participants that would seemingly increase workload, was that some participants believed more practice was needed across different age groups (DEd4, DEd6, OPP1). In addition, a couple of 
participants suggested that the program increase the ASL sign language course requirements (DEd4, OPP1). Again, the later, would increase the workload of teacher candidates.

Lesson plan writing was viewed as an important, yet time consuming part of practicum (1TC1) and student teaching (STP7). A 1st year candidate engaged in preschool practicum at the time of the study mentioned, "Writing out each lesson step by step is time consuming but so beneficial" (1TC1, p. 2). As previously reported, a deaf educator stated, "I think the consistency in expectations across practicum courses resulted in my continued improvement and growth in writing and executing lesson plans" (DEd4, p. 2). This same deaf educator also indicated that the large amount of work tied to practicum "sometimes...seemed like too much!" (DEd4, p. 2). This deaf educator did not single out lesson plan writing completely. Also, a preceptor noted that during off-site practicum, some teacher candidates were skilled at writing lesson plans and others were not (OPP1). Yet as previously mentioned at the student teaching level, most student teaching preceptors found Midwest teacher candidates were skilled in lesson plan writing (STP2, STP4, STP6, STP7).

One student teaching preceptor seemed conflicted with the lesson plan requirements during student teaching (STP7). She suggested striking a balance between the university requirements and what's required by the preceptor primarily by reducing the university requirements for lesson plans. From her perspective, "they [the teacher candidates] get so upset and overwhelmed...I've wiped a lot of tears" (STP7, p. 20, 396397). But then there was some hesitation, “you can't go back too far because, if you don't lay down the requirements, I don't think they look at what they're supposed to 
do... [and] I'm having problems right now with one [a teacher candidate from a different deaf education teacher preparation program] not thinking ahead...so I don't know if I really said anything now" (STP7, p. 21, 403-421).

Program teacher candidates were required to complete 150-200 clock hours of practicum prior to student teaching (FF1). Yet, two participants wished they had more hands-on practice earlier in the program (DEd4, OPP1), specifically at the undergraduate level (OPP1). More hands-on learning would increase requirements and teacher candidates' workload earlier in the program. Another deaf educator indicated that it would be helpful if teacher candidates were able to experience all major educational levels in PK-12 settings (DEd6). This was confirmed by a preceptor who said, "just because you do your student teaching in elementary, doesn't mean that you... find a job in elementary... so you...need to have a multi-level experience to... know what to expect" (OPP3, p. 20, 386-389). Again, the aforementioned suggestion for experiences across all settings could potentially increase program students' workload.

Lastly, as previously indicated student teaching preceptors commented on the teacher candidates' ability to teach students who communicated through oral or manual communication, including ASL. However, there were a couple of participants who suggested that the program increase its sign language requirement, most specifically in the area of ASL (DEd4, OPP1). As a result of this study, Midwest University may want to continually monitor the balance between coursework and practice. The program's comprehensive philosophy should continue to be a guidepost for meeting the needs of all students who are d/DHH. 
Exploring below the surface in teaching beyond academics. Teaching beyond academics was an emerging theme within the area of learning impact. This theme resonated with several participants (DEd7, OPP1, OPP2, PCF3, STP1, STP2, 2TC1). When including participants' perceptions of what a teacher candidate 'looked like' who was ready to teach students who were $\mathrm{d} / \mathrm{DHH}$, the data showed a marked distinction between groups by hearing status. All three $(100 \%)$ of the participants who were $\mathrm{d} / \mathrm{DHH}$ within the invited participant group echoed the importance of "teaching beyond academics' in theory, when discussing the ideal teacher candidate and/or as expressed within practice. In contrast, only seven $(28.0 \%)$ of the invited participants who were hearing echoed the teaching beyond academics theme.

The above mentioned difference in numbers between the participants who were $\mathrm{d} / \mathrm{DHH}$ and the participants who were hearing caught the researcher's attention. However, it was the content of the messages that were relayed by the participants who were $\mathrm{d} / \mathrm{DHH}$ that resonated at this researcher's core. The importance of powerful connections with others who were $\mathrm{d} / \mathrm{DHH}$, fitting in socially and independently in a hearing world, and one's journey toward embracing her identify as an individual who was hard of hearing were shared from different participants who were d/DHH.

The importance of teaching beyond academics was not reserved exclusively for participants who were d/DHH. There was a powerful quote (Taylor Swift quote) previously mentioned in the findings that was said by a preceptor who was hearing regarding the importance of students who were $\mathrm{d} / \mathrm{DHH}$ fitting in socially with students in the general classroom. In addition, a student teaching preceptor mentioned that teacher candidates that she worked with from Midwest University did not pretend to understand 
what it meant to be d/DHH (STP2). This preceptor further explained that a Deaf adult mentor within the classroom was able to be utilized if there was something that came up from a student who was d/DHH that was out of the teacher candidate's realm as a person with hearing.

As other participants who were hearing (OPP1, DEd4), the program faculty member who was d/DHH mentioned the use of interpreters (PCF3). However, this faculty member took it one step further. This faculty member's focused on the effective use of interpreters which included that teachers of students who are d/DHH need to make sure "not to have students [who are d/DHH] become dependent on them [interpreters]" (PCF3, p. 2). It was not enough that the students knew how to use the interpreter, this faculty member kept her eye on an ultimate goal of independence.

The aforementioned faculty member shared her personal story of contracting meningitis near one and a half years of age and her school journey once she received amplification at three years of age. She proudly mentioned how her mom, "didn't allow me to feel sorry for myself" (PCF3, p. 2) and how her parents made changes in her school placement when they saw her social skills lagging behind her peers. During much of this faculty member's educational experience, she was not around other students her age who were $\mathrm{d} / \mathrm{DHH}$. This faculty member stressed the importance of meeting one of her best friends, another student who was d/DHH, during her college years at this deaf education teacher preparation program. She relayed this sentiment about the significance of their friendship, "we automatically clicked as we shared similar interests/experiences... to this day, she is one of my best friends" (PCF3, p. 1). 
A final example was taken from a program student who was d/DHH; she shared her personal story. She was diagnosed with a bilateral hearing loss at the age of three years. She has used hearing aid technology consistently since that time. She recognized a personal change in her journey when during her tenure at Midwest University she commented, "[I] just recently accepted and embraced the fact that I am an individual who is hard of hearing" $(2 \mathrm{TC} 1, \mathrm{p} .1)$. This teacher candidate saw learning impact through Midwest University's encouragement of teacher candidates embedding exposure to d/Deafness for the students who are d/DHH they teach. Further research delving into the potential below the surface meaning of teaching beyond the classroom, may be warranted.

\section{Summary}

The findings of this phenomenological case study of the empowered and enabled learning (Marquardt, 2011) of Midwest University teacher candidates yielded a variety of dominant and emerging themes. One of the most compelling findings was that Midwest University's espoused philosophy was indeed practiced. The most prevalent themes, embracing diversity of $\mathrm{d} /$ Deafness and differentiated instruction were noted across all MO-DESE educator pillars: (a) commitment to the profession, (b) proficiency in practice, and (c) learning impact (MO-DESE, 2011, 2013). Midwest University teacher candidates were open-minded about communication options and were, in most situations, highly prepared to teach students who were d/DHH based on the student's primary mode of communication.

Another riveting research finding radiated from the data. Midwest University teacher candidates displayed an overwhelming commitment to the deaf education 
profession. Research question one resulted in no confounding evidence regarding the program's influence on empowering and enabling the learning of teacher candidates' commitment to the deaf education profession. The data from research question one revealed that committed deaf educators from Midwest University (a) embrace diversity of d/Deafness, (b) care passionately, (c) exceed high expectations, (d) are dedicated to learning, and (e) are immersed beyond the classroom in experiences with individuals who are $\mathrm{d} / \mathrm{DHH}$. The emerging theme indicated that committed deaf educators need to learn to collaborate with others.

Proficiency in Practice was the MO-DESE pillar that resulted in the most themes and, perhaps, showed the most complexity within the three MO-DESE pillars (MODESE, 2011, 2013). While 'how to teach' was the overarching theme echoed throughout all of the themes within this pillar, the 'how to teach' theme was not the most prevalent theme. The differentiated instruction theme rose to the top of this educator pillar. To answer research question two, it was clear that Midwest University teacher candidates developed proficiency in their teaching practice through coursework and experiences. However, proficiency in practice also resulted in some contradictory evidence regarding how the program empowered and enabled teacher candidates to learn proficiency in practice. The conflicting evidence revolved around navigating the public school system and subject content knowledge and methodologies.

Specifically, within MO-DESE's proficiency in practice pillar (MO-DESE, 2011, 2013), deaf educators from Midwest University (a) learn to differentiate instruction to meet different learners' needs, (b) learn the nuts and bolts of how to teach, in which the lesson planning process plays a critical role, (c) become highly prepared, (d) develop 
flexibility to make on-the-spot adjustments, (e) build teaching competence through practice, and (f) deepen their knowledge through coursework. The emerging themes indicated that proficient deaf educators need to (a) develop strong critical thinking skills, (b) implement evidence-based teaching, and (c) engage in an ongoing feedback loop for continued growth.

Research question three addressed learning impact, the third of MO-DESE's educator pillars, considered paramount for quality teachers (MO-DESE, 2011, 2013). Within the area of learning impact there were no dominant themes that emerged; however, meeting diverse learners' needs paralleled closely with the dominant differentiated instruction theme that emerged within proficiency in practice. It was challenging to succinctly divide those two areas. The emerging themes indicated that for Midwest University deaf educators to create learning impact in students who were $\mathrm{d} / \mathrm{DHH}$, the deaf educators need to: (a) meet learners' diverse needs, (b) collaborate with other educators, (c) teach beyond academics, and (d) connect with and engage the students. There was supporting evidence that all four of these emerging themes resonated through the program's preschool archival documents.

To answer research question four on how the program could improve to empower and enable teacher candidates who were ready to teach students who were $\mathrm{d} / \mathrm{DHH}$, the researcher was primarily interested in hearing the voices of the deaf educators and the student teaching preceptors. The voices of the deaf educators echoed through both the dominant and emerging program improvement themes. The dominant theme, public school 101, indicated that Midwest University should better prepare their teacher candidates to understand and navigate the public school system. The emerging theme, 
subject content knowledge and methodologies, indicated that Midwest University should increase the teacher candidates learning in specific subject content areas, most notably math, science, and reading. The area of reading yielded conflicting results. 


\section{CHAPTER FIVE}

\section{DISCUSSION}

At the time of this study, 15 (30\%) of the 50 states within the U.S. were allegedly without deaf education teacher preparation programs (Deaf Education Teacher Preparation Programs, 2012). Furthermore, deaf education teacher preparation programs were purportedly on the decline (Johnson, 2013) with additional deaf education programs at risk of closing their doors (Benedict, Johnson, and Antia, 2011). Similarly, a critical shortage of teachers of students who are d/Deaf or hard of hearing (d/DHH) was consistently reported within U.S. public schools (American Association for Employment in Education, 2008; U.S. Department of Education, 2012).

If the deaf education teacher preparation programs at risk of closing indeed close, the Midwest will be hit the hardest (Benedict et al., 2011). Program closures would result in 'a $40 \%$ decrease in the region's capacity to prepare teachers of deaf and hard of hearing students" (Benedict et al., 2011, p. 7). Program instability has further compromised meeting the need in public schools for educators of students who are d/DHH. The gap between students who are d/DHH in public schools and the teachers required to teach them needs to be filled.

There is a unique complexity to deaf education teacher preparation programs, communication philosophy. One essential element for national Council on Education of the Deaf (CED) approval is that teacher preparation programs design their curriculum to meet approved standards and align these standards with their espoused philosophy: (a) auditory oral, (b) bilingual-bicultural, or (c) comprehensive (CED, 2013). Therefore, teacher candidates leave programs prepared to teach students who are d/DHH based on 
the communication methodology used by the program. This difference among programs further complicates meeting the needs of students who are d/DHH in U.S. public schools.

Attention to the plight of deaf education resulted in a push for change in deaf education teacher preparation programs (Johnson, 2004, 2013; Lenihan, 2010).

Advances in hearing technologies and other technologies, early identification of hearing loss, and early intervention also sparked the need for change (Lenihan, 2010; Marvelli, 2010). It is imperative that deaf education teacher preparation programs that remain work to close the gap between the shortage of deaf educators and the need for deaf educators in public school systems across the country. Unfortunately, it will be the learners who are $\mathrm{d} /$ Deaf or hard of hearing and their families, the public school systems who serve the majority of students who are $\mathrm{d} / \mathrm{DHH}$, and ultimately, the greater community who will pay the price for not filling this need within U.S. schools.

At the time of this study, a majority (59.4\%) of deaf education teacher preparation programs self-identified as comprehensive philosophy programs (Deaf Education Teacher Preparation Programs, 2012). There was a gap in the literature of how deaf education teacher preparation programs function, especially those espousing a comprehensive philosophy. Even less was known about the functioning of these programs and how they prepared deaf education teacher candidates while simultaneously filling a community need by providing on-site services to learners who are $\mathrm{d} / \mathrm{DHH}$.

\section{Description of the Study}

The researcher conducted a qualitative, phenomenological case study of a Midwest, graduate, comprehensive deaf education teacher preparation program (SavinBaden \& Major, 2013). This study was bounded by one case, Midwest University, which 
was the pseudonym used for this deaf education teacher preparation program. It was also bounded by time, three academic years from August 2011 through May 2014. The researcher investigated this deaf education teacher preparation program and its teacher candidates. The phenomenon studied, empowered and enabled learning, was drawn from Marquardt's (2011) learning organization people subsystem.

In the field of deaf education, a field in which teacher preparation programs are struggling to survive (Benedict et al., 2011), it is critical that existing teacher preparation programs prepare teacher candidates who are able to positively impact student learning (MO-DESE, 2011). The researcher explored how Midwest University prepared teacher candidates who were "empowered and enabled to learn" (Marquardt, 2011, p. 26). This qualitative research focused specifically on the three professional educator pillars central to the development of quality teachers: (a) commitment to the profession (b) proficiency in practice, and (c) learning impact (MO-DESE, 2011). All of Missouri's quality indicators and teaching standards were encompassed within these three overarching frames (MO-DESE, 2011, 2013).

This study addressed the following four research questions:

1. How does the program empower and enable teacher candidates to learn commitment to the Deaf education profession?

2. How does the program empower and enable teacher candidates to learn proficiency in practice with students who are d/Deaf or hard of hearing?

3. How does the program empower and enable teacher candidates to create learning impact in students who are $\mathrm{d} /$ Deaf or hard of hearing? 
4. How could the program improve to empower and enable the learning of teacher candidates who are prepared to teach students who are d/Deaf or hard of hearing? For this particular study, the best way to maximize sampling variation was to include, in as much as possible, all eligible participants as bounded by this one case and three year time span (Hatch, 2002). There were 44 potential participants. Thirty-four were invited participants and up to 10 potential, unique preschool family participants were included through archival documents. The participants encompassed both internal and external stakeholders whom Marquardt (2011) espoused were important for organizational learning.

Invited participants included the following three general groups: (a) program students, (b) program faculty, and (c) preceptors. Of the 34 invited participants, 32 (94.1\%) agreed to participate, and $28(87.5 \%)$ actually participated. The percentage of participation was fairly equally distributed across all general groups with a range of $81.8 \%-87.5 \%$. The 10 possible unique preschool families were not included in the percentages because there was no way to verify the exact number that participated.

Of the 28 actual participants, there was one participant who was invited to participate as a preceptor and a program faculty because this individual was a part of both groups. The individual participated in one of the two categories. Therefore, although, non-participation of this individual as program faculty dropped the percentage of participation in that group, it did not drop participation of the total invited participants. No individual was counted more than once in the total number of study participants. In essence, the voice of this participant was reflected within the study; thereby, the overall actual participation rate was $87.5 \%$. 
Data was collected through (a) surveys, (b) interviews, and (c) archival documents which enhanced the research design (Hatch, 2002). Program students which consisted of (a) 1st year teacher candidates, (b) 2nd year teacher candidates, and (c) deaf educators received surveys. Program faculty which included (a) full-time faculty, and (b) per course faculty also received surveys. Preceptors included (a) off-site preceptors, and (b) student teaching preceptors. They were interviewed by the researcher.

\section{Context of the Study}

At the time of this study, the researcher was a clinical professor at Midwest University. The researcher had been teaching in this deaf education program for 22 years; therefore, the researcher took extra care to ascertain that the participants understood that this study was voluntary. The voluntary component was explicit in the consent forms as well as emails that were sent to participants. In addition, participants received no more than one recruitment email reminder and one completion reminder during the course of the study unless a participant contacted the researcher directly. In that case, the researcher complied with the request of the participant, for example, to resend the survey.

A complete description of the context of the study: (a) program setting, (b) participants, and (c) students who were d/DHH they served was important so that practitioners could determine applicability and transferability of the findings to their settings (Mertens, 2015). At the time of this study, Midwest University was the only deaf education program in the state with a comprehensive philosophy (Deaf Education Teacher Preparation Programs, 2012). Midwest University is located within a state in 
which three of the four primary bordering states had no existing deaf education programs (Deaf Education Teacher Preparation Programs, 2012).

The participant pool was primarily White, hearing, and female. Of the potential 44 participants, four $(9.0 \%)$ or five $(11.4 \%)$ were $\mathrm{d} / \mathrm{DHH}$. The possible fifth person was a parent of the 10 unique preschool families who was $\mathrm{d} / \mathrm{DHH}$. However, the Preschool Evaluation of Services archival documents (PED1-PED26) were completed anonymously so there was no way to know for sure that that individual participated. Of the 34 invited participants, three who were d/DHH actually participated. Two of the three individuals self-identified as deaf; one self-identified as hard of hearing.

Although the participant pool of this study was quite homogeneous in the aforementioned areas, the actual pool of participants was also quite diverse in other areas. The following data was reported using the total number of actual participants from the pool of 28 invited participants who chose to participate. Program faculty represented $17.9 \%$ (5 out of 28 ) of the invited participant pool. They held at least master's degrees in one or more of the following areas: (a) deaf education, (b) counseling, and (c) vocational rehabilitation. One faculty had a Ph.D. One faculty member was also a certified interpreter. The program faculty who had taught students who were d/DHH included experiences that ranged from early childhood through 12th grade. In addition, one faculty member was a CODA, child of deaf adults.

Program students represented 50\% (14 out of 28) of the invited participant pool. Seven of the students had bachelor degrees and were in the process of completing a master's degree. Eight had a master's degree. The program students were equally divided between traditional masters and accelerated master's options. These individuals 
were the primary participants of this qualitative study which explored the program's influence on their empowered and enabled learning (Marquardt, 2011) across the MODESE educator pillars (MO-DESE, 2011, 2013).

The preceptors represented $32.1 \%$ ( 9 out of 28 ) of the invited participant pool. Six $(66.7 \%)$ preceptors were program graduates; the remaining three (33.3\%) were not program graduates. However, the majority $(60 \%)$ or 3 out of the 5 student teaching preceptors were not program graduates. And the group of student teaching preceptors had the most comprehensive and complete knowledge of the program teacher candidates' performance teaching students who were $\mathrm{d} / \mathrm{DHH}$ at the time the teacher candidates graduated from the program.

The majority (77.8\%) of preceptors taught within the public school system; $22.2 \%$ of the preceptors taught at a School for the Deaf. The preceptors taught in two different states and their schools were located in three of the four general locale categories: (a) "rural", (b) "town", (c) "suburban", and (d) "city" (National Center for Education Statistics, 2012, p. 1). There was no preceptor's school that fit within the town classification. All program students had practicum and/or student teaching experiences in rural and city locales; some had placements in suburban school locales as well. The preceptors were also diverse in types of teaching positions from an itinerant position covering multiple rural districts to a self-contained classroom at a School for the Deaf and variations in between. Collectively, the preceptors had over 100 years of experience teaching students who were $\mathrm{d} / \mathrm{DHH}$.

The population of students who were $\mathrm{d} / \mathrm{DHH}$ served by the participants at the time of data collection, numbered somewhere between 93 and 105. There were some 
variations in data reported from question to question. The majority of these students were White. This population, however, was quite diverse when considering mode of communication, amplification, and whether or not the students who were $\mathrm{d} / \mathrm{DHH}$ had an additional condition or disability. Most students who were d/DHH used spoken language only to communicate. Sign language with spoken language was the next most frequently occurring primary mode of communication. The smallest percentage of students who were $\mathrm{d} / \mathrm{DHH}$ was reported to use ASL as their primary language.

Most (75\%) of the schools included students who were $\mathrm{d} / \mathrm{DHH}$ whose primary mode of communication encompassed at least two, if not all three of the primary communication modality categories. Six schools served students who represented two different primary modes of communication and six schools served students which included all three primary modes of communication categories. There were only two schools, at the time of the study, who were only serving students whose primary mode of communication was spoken language. And there were only two schools, at the time of this study, who were only serving students whose primary communication mode was sign language with spoken language.

Of the students who were $\mathrm{d} / \mathrm{DHH}$, over half used hearing aid technology, nearly $25 \%$ used cochlear implant technology, and less than $10 \%$ used a combination of the two hearing technologies. There were an equal number of students who were $\mathrm{d} / \mathrm{DHH}$ who used no amplification as students who used one cochlear implant and one hearing aid. A limited number of students fell within the category of using bone conduction technology or 'other' technology. Approximately, 70\% percent of the students who were d/DHH were reported to have no additional disabilities with the other approximately $30 \%$ 
reported to have additional disabilities. The students who were $\mathrm{d} / \mathrm{DHH}$, served by participants of this study, formed an eclectic group.

\section{Summary of Findings}

Marquardt's (2011) empowered and enabled learning phenomenon guided the research questions for this phenomenological case study. The first three research questions were constructed using MO-DESE's three educator pillars deemed essential for the development of quality teachers: (a) commitment to the profession, (b) proficiency in practice, and (c) learning impact (MO-DESE, 2011, 2013). The final research question focused on program improvement. There was a participant completion rate of over $82 \%$ of all internal and external stakeholders invited to participate. A variety of themes emerged from the voices of the participants. Following was a summary of the themes organized by research question.

\section{Research Question One}

How does the program empower and enable teacher candidates to learn commitment to the deaf education profession?

Dominant themes of commitment. The data revealed that teacher candidates from Midwest University displayed an unwavering commitment to the deaf education profession. This commitment to the profession was reflected in deaf education teacher candidates who (a) embrace diversity of d/Deafness, (b) care passionately, (c) exceed high expectations, (d) are dedicated to learning, and (e) are immersed 'beyond the classroom' with individuals who are d/DHH. Embracing diversity of d/Deafness, the first and most prevalent dominant theme of commitment, shared equal billing to the most prevalent dominant theme, differentiated instruction, of research question two. These 
two themes resonated with more research participants than any other themes that surfaced in this study and were the hallmark of this program's comprehensive philosophy.

Embrace diversity of d/Deafness. Midwest University was unbiased about modes of communication and advocated for parent choice and child's needs. This embracing diversity of $\mathrm{d} /$ Deafness theme was reflected in teacher candidates' openness to different modes of communication for individuals who were $\mathrm{d} / \mathrm{DHH}$. It was also forthcoming in their ability to meet the varying communication modality needs of students who were d/DHH within the teacher candidates' school placements.

Care passionately. All internal and external participant groups exuded the compassionate caring theme. Passionate caring was so universal among the participants that their voices seemed to convey that this aspect of commitment was bigger than the program. Many of the participants had found their calling, not career, when they discovered the field of deaf education.

Exceed high expectations. Program faculty had high expectations for their teacher candidates and the teacher candidates delivered. All general participant groups recognized this 'above and beyond' expectations' commitment theme. This theme manifested an ultimate focus on the students who were d/DHH they served.

Are dedicated to learning. Teacher candidates were reported to be dedicated to learning across all three general, invited participant groups. Commitment through this dedication to learning theme needed to be ongoing. Midwest University jump started this dedication to learning through resources provided, connections to others for continued learning, and a variety of hands-on learning opportunities through program projects. 
Are immersed 'beyond the classroom' with individuals who are d/DHH. This 'beyond the classroom' commitment transpired through hands-on practicum experiences with individuals who were $\mathrm{d} / \mathrm{DHH}$ which consistently resulted in program clock hours above state and frequently national CED requirements. In addition, program faculty encouraged and, at times, required course projects or experiences in which teacher candidates engaged within the Deaf Community and greater community of individuals who were $\mathrm{d} / \mathrm{DHH}$. This push to engage teacher candidates was touted as a positive program influence by all three general participant groups.

Emerging themes of commitment. An emerging theme also surfaced within the area of commitment. The emerging theme showed some evidence of the importance of teacher candidates learning to collaborate to learn commitment. No conflicting evidence surfaced on how the program empowered and enabled teacher candidates to learn commitment to the profession.

Learning to collaborate. As with the dominant commitment themes, this learning to collaborate emerging theme was heard from all three general, invited participant groups. Collaboration signaled one way in which Midwest University influenced teacher candidates' commitment within the deaf education profession. Program faculty and preceptors emphasized the importance of collaboration.

\section{Research Question Two}

How does the program empower and enable teacher candidates to learn proficiency in practice with students who are d/Deaf or hard of hearing?

Dominant themes of proficiency in practice. The data revealed that teacher candidates from Midwest University displayed proficiency in practice. Proficiency in 
practice was reflected in deaf education teacher candidates who (a) learn to 'differentiate instruction' to meet diverse learners' needs (b) learn 'how to teach' in which the lesson planning process is critical, (c) become highly prepared, (d) develop flexibility to make on-the-spot adjustments, (e) build teaching competence through practice, and (f) deepen knowledge through coursework. Differentiated instruction which coincided with the embracing diversity of d/Deafness, commitment theme, was the most prevalent theme within research question two.

\section{Learn to 'differentiate instruction' to meet diverse learners' needs.}

Communicating in the student's primary mode of communication was an important component to differentiate instruction. However, differentiated instruction included other critical aspects in order to be proficient in teaching. Individualizing students' learning needs required teacher candidates to understand learners' needs and plan accordingly.

Learn 'how to teach' in which the lesson planning process is critical. 'How to teach' was the reality that resonated throughout each of the dominant and emerging themes within proficiency in practice. Learning the lesson planning process was pivotal for teacher candidates to learn 'how to teach'. The programs directed focus on lesson planning writing, implementation, and reflection on the learning resulted in strong lesson plan writing skills of teacher candidates across student teaching placements.

Become highly prepared. Midwest University teacher candidates become highly prepared throughout their deaf education teacher preparation program. The most compelling evidence emerged from student teaching preceptors who were not program graduates. Not only did they proclaim the teacher candidates were highly prepared, at times, they compared Midwest University students to students in other deaf education 
teacher preparation programs. In one case, a preceptor even compared her preparation at another deaf education teacher preparation program. In all examples, Midwest University teacher candidates came out on top in preparedness.

Develop flexibility to make on-the-spot adjustments. Another dominant theme, flexibility, was an essential skill recognized by all three general invited participant groups. Teacher candidates demonstrated flexibility when they were able to monitor and adjust throughout their lessons and day. Flexibility was practiced in a variety of settings beginning with the on-site preschool. Again, the student teaching preceptors who did not graduate from Midwest University were some of the strongest voices touting the flexibility of teacher candidates from this deaf education teacher preparation program.

Build teaching competence through practice. Midwest University integrated book knowledge with practice. On-site and off-site practicum was deemed critical for the development of teaching competence in teacher candidates and was a dominant theme within proficiency in practice. Midwest University's practicum experiences were sequential and front-loaded with skill development within the program's preschool. Building competence also included observations, watching experienced teachers teach. Deepen knowledge through coursework. As previously mentioned, practicum and book knowledge go hand in hand. And as practicum, coursework knowledge was deemed critical for the development of teaching competence in teacher candidates. Both building teaching competence through practice and deepening knowledge through coursework were the least prevalent of the dominant themes. Knowledge in the area of d/Deafness was noted as a program strength. 
Emerging themes of proficiency in practice. Three emerging themes also surfaced within the area of proficiency in practice. The emerging themes showed some evidence of the importance of teacher candidates: (a) developing strong critical thinking skills, (b) implementing evidence-based teaching, and (c) engaging in an ongoing feedback loop for continued growth to build proficiency in practice. There was some conflicting evidence that surfaced on how the program empowered and enabled teacher candidates to learn proficiency in practice. Teacher candidates needed a better understanding of the public school system and an increase in knowledge and methodologies related to subject content areas. This evidence was shared under research question four on program improvement.

Developing strong critical thinking skills. Teacher candidates were required to critically reflect on their lessons and teaching practice. Critical reflection included looking back on what went well and looking ahead to what could be changed to improve the lesson. Teacher candidates' critical thinking was also expected while developing the lesson plan. They were expected to anticipate potential challenges and think through potential solutions.

Implementing evidence-based teaching. The evidence-based teaching theme was heard from some of the participants, primarily, the deaf educators, who were in their first or second year of teaching. The programs focus on evidence-based teaching was noted by a faculty member. Assessment needed to be connected to teaching.

Engaging in an ongoing feedback loop for continued growth. Teacher candidates received ongoing feedback from professors as well as preceptors. Within 
practicum, feedback included both qualitative and quantitative feedback. Teacher candidates were expected to make adjustments when needed based on feedback received.

\section{Research Question Three}

How does the program empower and enable teacher candidates to create learning impact in students who are d/Deaf or hard of hearing?

Dominant themes of learning impact. The data did not reveal any dominant themes within the area of learning impact. The first emerging theme, meeting diverse learners' needs, however, was closely tied to the differentiated instruction theme in research question two. The researcher attempted to separate these two areas; the same data was not used to answer both research question two and research question three.

Emerging themes of learning impact. Four emerging themes surfaced within the area of learning impact. The emerging themes showed some evidence of the importance of teacher candidates: (a) meeting diverse learners' needs, (b) collaborating with educators, (c) teaching beyond academics, and (d) connecting with and engaging students to create learning impact in students who are d/DHH. Preschool Evaluation of Services documents provided supporting documentation for learning impact within all four of the emerging themes.

Meeting diverse learners' needs. Meeting diverse learners' needs emerging theme was demonstrated through examples, such as a district purchasing FM systems for classrooms to use of Visual Phonics. Preschool archival documents showed the most prevalent learning of the preschoolers was centered on communication modalities. Different modes of communication were highlighted on different documents. 
Collaborating with educators. Collaborating with educators on off-site placements resulted in learning by both teacher candidates and preceptors. This collaborative learning resulted in some mutual benefits. There was an exchange of ideas and resources to benefit students who were $\mathrm{d} / \mathrm{DHH}$ they served.

Teaching beyond academics. Teaching beyond academics surfaced from a few individuals. What emerged from the data illuminated different angles on areas of learning and connecting at school beyond core subjects and related to $\mathrm{d} /$ Deafness. There was a sense of the important role deaf educators' play in teaching cultural awareness of $\mathrm{d} /$ Deafness as well as skills for navigating socially and independently within a hearing world. Within the preschool archival documents, parents mentioned the importance of their children learning to interact with others.

Connecting with and engaging students. Teacher candidates who connected with the students who were $\mathrm{d} / \mathrm{DHH}$ and had engaging lessons were creating learning impact in the students. Students were not even aware they were learning. They were eager to come back for more. Connections were developed by getting to know the students and capitalizing on the students' interests. Preschool archival documents reiterated how the preschoolers wanted to go to school. The preschoolers were connected and engaged.

\section{Research Question Four}

How could the program improve to empower and enable the learning of teacher candidates who are prepared to teach students who are d/Deaf or hard of hearing?

Dominant themes of program improvement. The data revealed that there was one dominant theme related to program improvement. This theme demonstrated evidence of the need for program improvement at Midwest University. Midwest University could 
improve in empowering and enabling the learning of teacher candidates in navigating the public school system.

Navigating the public school system. The deaf educators, recent program graduates in their first or second year of teaching at the time of this study, were the primary participants whose voices were echoed throughout this dominant theme, public school 101. A majority of the deaf educators expressed a need for program improvement focused on enhanced knowledge and/or experiences within the public schools. Some public school topics were focused on deafness related topics such as working with interpreters and learning about the challenges and solutions in itinerant placements. Other topics were varied, such as understanding the special education process and understanding the general education curriculum.

Emerging themes of program improvement. One emerging theme surfaced within the area of program improvement. The emerging theme showed some evidence of possible program improvement needed for increasing teacher candidates' subject content knowledge and teaching methodologies. As with the dominant theme, the majority of the deaf educators were the participants who echoed this emerging theme of program improvement.

Increasing subject content knowledge and methodologies. Increased knowledge in math, science, and reading were the most frequently mentioned content subject needs. Reading, however, yielded contrary evidence. A majority of the deaf educators believed they needed more program preparation in subject content and methodologies. 


\section{Conclusions}

The dominant themes within research questions one and two, commitment to the profession and proficiency in practice, displayed the most compelling evidence of teacher candidates empowered and enabled learning through this deaf education teacher preparation program. The most prevalent theme stretched across all three of MO-DESE's three educator pillars (MO-DESE, 2011, 2013) and reflected the prominence of diversity of $\mathrm{d} /$ Deafness across all three general participant groups. This theme stressed the importance of meeting the individualized needs of the diverse population of students who were d/DHH beginning with the student's mode of communication. The espoused comprehensive philosophy of Midwest University was clearly demonstrated in the resounding theme of diversity of $\mathrm{d} /$ Deafness.

There were also emerging themes that were heard through the voices of participants within the educator pillars. The emerging themes surfaced, primarily, within research questions two and three, proficiency in practice and learning impact. The emerging themes may provide opportunities for program improvement to empower and enable the learning of teacher candidates within those areas. The emerging themes were shared within the context of research question four on program improvement.

\section{Research Question One: Commitment to the Profession}

The program resulted in teacher candidates who radiated commitment to the deaf education profession. Midwest University teacher candidates (a) embraced diversity of d/Deafness, (b) cared passionately, (c) exceeded high expectations, (d) were dedicated to learning, and (e) were immersed 'beyond the classroom' with individuals who are $\mathrm{d} / \mathrm{DHH}$. The program faculty modeled openness to diversity of $\mathrm{d} /$ Deafness. This was 
evident through coursework and experiences that prepared teacher candidates to meet the communication needs of students who were d/DHH using the students' primary modes of communication. Midwest University offered sign language courses, four in ASL and two in CASE. Diversity of d/Deafness also extended into welcoming preschoolers who were $\mathrm{d} / \mathrm{DHH}$ in their program preschool regardless of mode of communication, use or non-use of hearing technology, and the presence of additional disabilities.

Passionate caring was another universal theme that radiated from participants. Their voices seemed to convey that commitment was bigger than the program. Many of the participants, including faculty, seemed to have found their calling, not career, when they discovered the field of deaf education. Midwest University faculty's above and beyond expectations and dedication to learning influenced the empowered and enabled learning of teacher candidates. Those themes were recognized and realized in the teacher candidates across all general participant groups.

Lastly, program students became more committed with increased exposure and involvement in the Deaf Community and greater community of individuals who are $\mathrm{d} / \mathrm{DHH}$. Program faculty required and encouraged this involvement through course assignments, collaborative, action research-based projects, and practicum experiences. There was no conflicting evidence of empowered and enabled learning of program students to learn commitment to the deaf education profession.

\section{Research Question Two: Proficiency in Practice}

The program shaped teacher candidates who displayed strong proficiency in their teaching practice. Midwest University teacher candidates (a) learned to 'differentiate instruction' to meet diverse learners' needs, (b) learned 'how to teach' with a focus on the 
lesson planning process, (c) became highly prepared, (d) developed flexibility to make adjustments when teaching, (e) built teaching competence through practice, and (f) deepened their knowledge through coursework. Differentiated instruction was reflected within the most prevalent theme, diversity of $\mathrm{d} /$ Deafness. This part of differentiated instruction focused on meeting the diverse communication needs of the students.

Practicum experiences provided by program faculty focused on the lesson planning process from writing and implementing lesson plans to critical reflections within the lesson process. Learning the lesson planning process took more time for some teacher candidates than others; however, the sequential practicum process paid off. Four of the five student teaching preceptors touted the strong lesson writing skills of Midwest University teacher candidates.

Student teaching preceptors were not always sure what the program had done to empower and enable the teacher candidates to be proficient in practice; however, all three preceptors who were not program graduates sang the praises of teacher candidates' preparedness and flexibility. In fact, some believed the teacher candidates were more prepared and flexible than any other student teachers they had from other programs.

Lastly, the program prepared teacher candidates to be proficient in their practice through an integration of coursework knowledge and practice. Graduate teacher candidates were enrolled in practicum four consecutive semesters prior to student teaching. In their fifth and final semester, teacher candidates were enrolled in a full-day, student teaching placement which covered 11-13 calendar weeks. 


\section{Research Question Three: Learning Impact}

MO-DESE's learning impact educator pillar did not yield any dominant themes within this study. The emerging themes may support the need for program improvement. The emerging themes, with the exception of teaching to the learners' diverse needs, are presented below under research question four.

\section{Research Question Four: Program Improvement}

One dominant theme that emerged from the data was navigating the public school system, 'public school 101'. Public school 101 was the most prevalent theme that depicted the need for program improvement. Almost every deaf educator indicated that public school knowledge was the primary gap experienced by deaf educators within their preparation at Midwest University. In addition, an emerging theme of program improvement surfaced.

Again, deaf educators lead the charge in uncovering the potential area of program improvement in subject content knowledge and methodologies. Although the emerging theme of subject content knowledge and methodologies resonated with six different participants, only two participants per area mentioned the specific content areas of need related to math, science, and reading. Within the area of reading, there was evidence that also supported program learning within the area of reading and studying reading research. Only one person suggested a need in learning more history content.

The aforementioned themes were specific program improvement themes that emerged from the data provided by invited research participants. The subsequent themes, all emerging themes, were specific positive aspects of the program also identified by research participants. Since these upcoming emerging positive themes were not 
considered strong enough to be dominant themes, the researcher believed these themes should be of consideration for continued growth within the program. Figure 2 on the subsequent page depicted the program's improvement dominant and emerging themes that were heard from the participants, particularly the deaf educators. In addition, Figure 2 included the emerging positive themes that surfaced to answer research questions one, two, and three.

There may be more that the program can do to support the teacher candidates becoming empowered and enabled to learn, primarily within the emerging proficiency in practice, and learning impact pillars. Meeting diverse learners' needs was not believed to be a potential area of improvement because its emerging status in learning impact was connected to not using the same data to support proficiency in practice and learning impact. Furthermore, diversity of d/Deafness and differentiated instruction were the strongest themes supported by the research. These two themes, in part, were encompassed within the meeting diverse learners' needs of research question three.

There were seven additional positive program emerging themes that may be considered for program improvement. The first four themes were heard from six participants. The final three themes were heard by five participants each. The emerging themes were: (a) learning to collaborate, (b) developing critical thinking skills, (c) collaborating with educators (d) learning beyond academics, (e) implementing evidencebased teaching, (d) learning through ongoing feedback loop, and (c) connecting with and engaging students. 


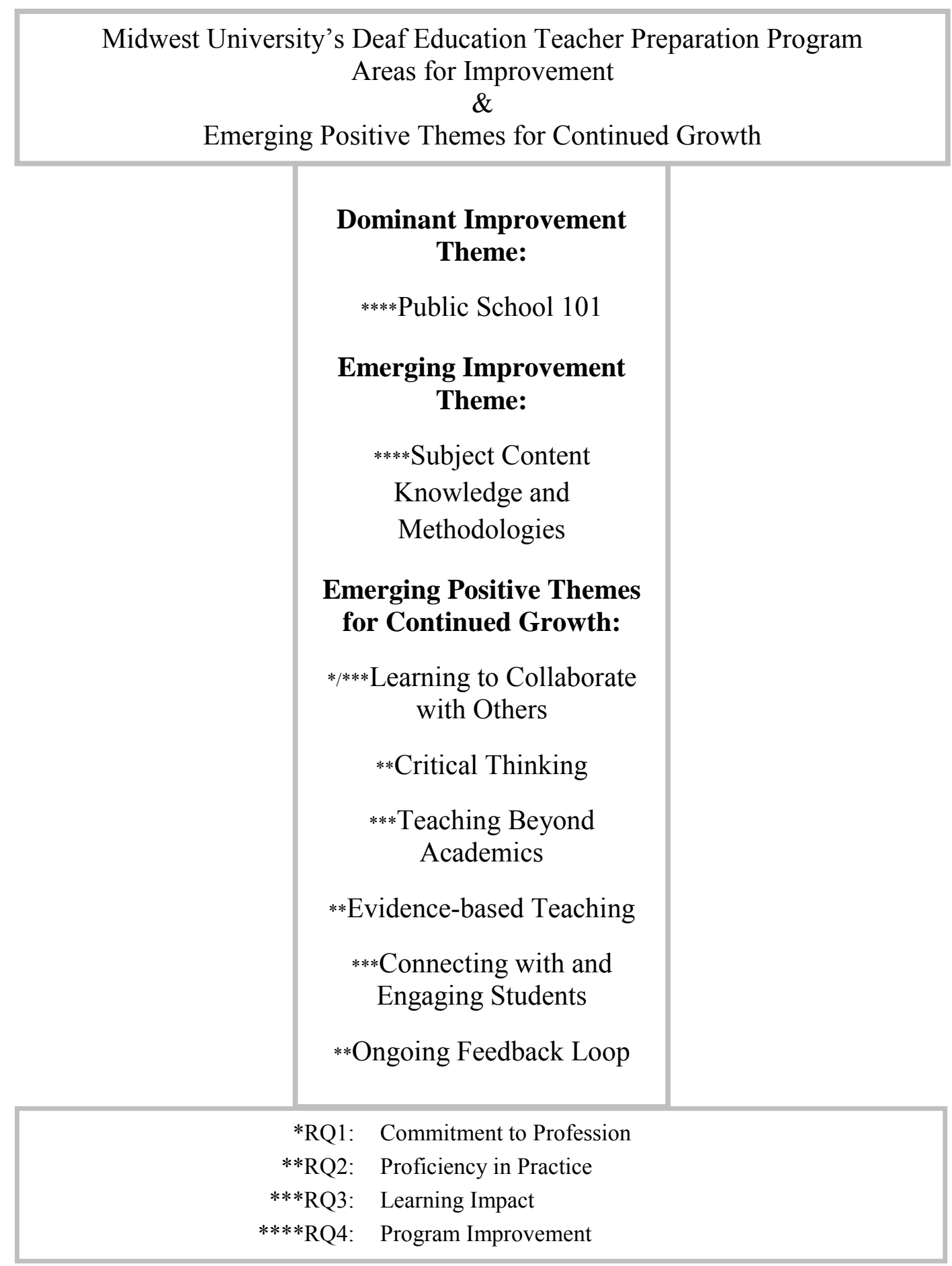

Figure 2. Study Findings of Areas for Improvement Themes and Areas for Continued Growth in Order of Prevalence and Keyed by Research Question 


\section{Discussion of the Findings}

An impressive $82.4 \%$ of all possible invited participants actually completed participation in this phenomenological case study. When considering only those participants who agreed to participate, this rate climbed to $87.5 \%$. This excellent participant completion rate increased confidence in the results that emerged from the study. It may also further indicate the strong commitment participants have to this deaf education teacher preparation program. At the very least, it seems that it would indicate confirmation in the first pillar, commitment to the profession, of all participants.

The findings within the current study showed alignment with some related literature in the field of deaf education. This study also displayed contrary evidence of alignment with current literature. Within the discussion of the findings, the researcher provided information on the extent to which this study aligned with and contradicted other research in the field. Next, the researcher discussed the usefulness of the Marquardt's (2011) people subsystem learning organization framework to understand the empowered and enabled learning of Midwest University teacher candidates.

\section{Evidence of Alignment and Non-alignment with Related Literature}

The oral-manual controversy has been long-standing within the field of deaf education (Marschark, Lang, \& Albertini, 2002). The medical perspective viewed deafness through a lens of deficit, indicating that hearing loss should be fixed or cured (Crittenden, 1993; Moores, 2001; Paul \& Whitelaw, 2011). The sociocultural model embraced deafness as a difference (Moores, 2001) rather than as a disability (Schirmer, 2001). Paul and Whitelaw (2011) saw value in both the medical and sociocultural 
perspectives. As Paul and Whitelaw (2011), the deaf education teacher preparation program of this current study embraced diversity of $\mathrm{d} /$ Deafness.

According to this study, Midwest University exemplified a comprehensive philosophy (CED, 2013). Program faculty modeled an unbiased approach to modes of communication for individuals who were d/DHH. Teacher candidates received coursework and experiences in manual communications, ASL and CASE, as well as listening and spoken language. During the course of this study, teacher candidates taught students who were $\mathrm{d} / \mathrm{DHH}$ who used a variety of modes of communication and in a variety of settings. Furthermore, the program's preschool for children who are d/DHH welcomed children regardless of mode of communication, use or non-use of amplification, and the presence of additional disabilities (Wang et al., 2014).

The demographics of the population of students who were $\mathrm{d} / \mathrm{DHH}$ of this current study somewhat mirrored the demographics in Gallaudet Research Institute's (GRI) 2009-2010 Annual Survey of Deaf and Hard-of-Hearing Children and Youth (GRI, 2011). In addition, the demographics of the teachers of students who were $\mathrm{d} / \mathrm{DHH}$ within this study reflect the national trend (Ausbrooks, Baker, \& Daugaard, 2012; Simms, Rusher, Andrews, \& Coryell, 2008). This similarity in demographics of the students who are $\mathrm{d} / \mathrm{DHH}$ and the teachers who serve them was important to know for understanding the applicability of the study's findings beyond the Midwest.

Similarities surfaced in modes of communication of students who were d/DHH. GRI (2011) data indicated that 53\% of the students who responded to the annual survey used spoken language only as their communication modality. This study revealed that $55.2 \%$ of the students' primary mode of communication was spoken language. The data 
on sign language was reversed when comparing this study with the GRI 2009-2010 data. GRI data indicated that $12 \%$ of the students who were $\mathrm{d} / \mathrm{DHH}$ reported using sign language with spoken language and $27 \%$ used sign language only to communicate. The current research showed $28.1 \%$ of the students using sign language and spoken language and only $16.7 \%$ using ASL as their primary mode of communication.

According to GRI (2011) data, for those students responding to the question on additional conditions or disabilities, $39 \%$ reported they had at least one additional condition. Within this current study, $28.6 \%$ of the students who were $\mathrm{d} / \mathrm{DHH}$ were reported to exhibit an additional disability. Mitchell and Karchmer (2006) and Spencer and Marschark (2010) purported that students who were d/DHH in public schools may be underrepresented in the GRI data. It is estimated that at least 35\% up to possibly over $50 \%$ of students who are d/DHH may have an additional condition or disability (HoldenPitt \& Diaz, 1998; Mitchell \& Karchmer, 2006; Spencer \& Marschark, 2010).

Holden-Pitt and Diaz (1998) showed a shift in racial and ethnic backgrounds of students who were d/DHH using GRI survey data during a 20 year period, ending in 1997. In $1997,58 \%$ of the population of students who were $\mathrm{d} / \mathrm{DHH}$ was White, $18 \%$ was Hispanic, $17 \%$ was African American or Black and 4\% was Asian. The population of students who were d/DHH associated with this current study was reported to be $64.4 \%$ White, 18.8\% Hispanic or Latino, and 8.9\% African American. A few other racial and ethnic backgrounds were reported, however, the percentages were below the one-tenth percentage level. This current study showed somewhat comparable demographics within the area of race and ethnicity. 
Similarly, teacher demographics of the Midwest University study were consistent with other teachers of students who were d/DHH as reported by Ausbrooks et al. (2012) and Simms et al. (2008). Ausbrooks et al. (2012) found that most teachers of students who were $\mathrm{d} / \mathrm{DHH}$ were White, female, and hearing. In essence, the overall demographics of the students who were $\mathrm{d} / \mathrm{DHH}$ reported in this current study seemed to somewhat parallel the population of students who were d/DHH reported through GRI data (HoldenPitt \& Diaz, 1998; GRI, 2011). Likewise, teachers of students who were d/DHH mirrored the typical population (Ausbrooks et al, 2012; Simms et al, 2008). Again, the similarities of demographics between the current study and national data may enhance the transferability of this study's findings beyond the Midwest region.

Teachers of students who are d/DHH were reported to need a variety of skills and experiences (Dodd \& Sheetz, 2003; Johnson, 2013; Luckner \& Howell, 2002; Teller \& Harney, 2005/2006). Johnson (2013) completed a review of a variety of studies and summarized the knowledge and skill sets needed by teachers of students who were d/DHH. Johnson (2013) reported that teachers needed to stay abreast of advances in hearing technologies and the development of listening and spoken language as well as the importance of having sign language proficiency. Midwest University prepared teacher candidates who were shown to be proficient in their teaching of students who were $\mathrm{d} / \mathrm{DHH}$ regardless of whether students' communication modes were oral or manual or used a combination of methodologies, including Visual Phonics.

Johnson (2013) also reported that teachers needed to have knowledge of service delivery across a wide span of school environments and ages. This included early intervention, center-based programs, itinerant education, residential schools, and 
education in the general classroom, and secondary transition. Midwest University teacher candidates had direct experiences in schools that met three of the four locale categories as identified by the U.S. National Center for Education Statistics (National Center for Education Statistics, 2012). And all Midwest University teacher candidates had experiences in a center-based program and public school placements.

Only some of the teacher candidates received practicum or student teaching experiences in Schools for the Deaf and itinerant placements. Luckner and Howell (2002) reported that itinerant teaching placements for teachers of students who are $\mathrm{d} /$ DHH have increased. In this current study, one deaf educator reported that understanding the role, challenges and solutions in itinerant placements was so important in navigating the public school system that she believed it should be its own class within Midwest University.

A majority of Midwest University teacher candidates had no direct experience in early intervention services for young children who were $\mathrm{d} / \mathrm{DHH}$ between the ages of birth and three years. Since spring 2011, parent-infant services for infants and toddlers who were $\mathrm{d} / \mathrm{DHH}$ and their families were no longer available through Midwest University. This greatly limited Midwest University teacher candidates' exposure to the world of early intervention with children who were d/DHH below the age of three years. Early intervention has been found to play a critical role in early language development of young children who are d/DHH (Joint Committee on Infant Hearing, 2013; MeinzenDerr, Wiley, \& Choo, 2011; Spencer \& Marschark, 2010; Yoshinaga-Itano, 2003). It has also been found that regardless of the child's mode of communication, it is imperative 
that early intervention services begin directly following the diagnosis of hearing loss (JCIH, 2013; Wang \& Engler, 2011; Yoshinaga-Itano, 2003).

The aforementioned gap in parent-infant or early intervention (Birth - 3 years) service delivery at Midwest University was not mentioned by any of the participants within the current study. Early intervention for infants and toddlers who are d/DHH and their families is essential (JCIH, 2013; Lenihan, 2010; Marvelli, 2010; Spencer \& Marschark, 2010; Wang \& Engler, 2011; Yoshinaga-Itano, 2003). Midwest University teacher candidates may require more than just knowledge in this area to be prepared to meet the needs of young children (Birth -3 years) who are $\mathrm{d} / \mathrm{DHH}$ and their families at this critical juncture.

In Johnson's (2013) review of research, he reported that teachers of students who were $\mathrm{d} / \mathrm{DHH}$ needed to engage in evidence-based teaching, develop collaborative skills, and develop their students' self-advocacy skills. Each of these areas was identified as an emerging theme within this current study. Midwest University teacher candidates seemed to be developing these skill sets, although, further focus may be warranted since these skills did not reach dominant theme status. Johnson (2013) and others (Dodd \& Scheetz, 2003; Luckner \& Howell, 2002; Teller \& Harney, 2005/2006) identified “an every-expanding array of knowledge, skills and experiences” (Johnson, 2013, p. 441) necessary for educators to teach students who are d/DHH. The previous couple of pages recapped some of the knowledge and skills needed.

\section{Usefulness of the Conceptual Framework of the Study}

Marquardt's learning organization's empowered and enabled people subsystem was used to guide this phenomenological case study (2011). Learning is critical to 
organizational viability (Marquardt, 2011; Senge, Roberts, Ross, Smith, \& Kleiner, 1994; Senge, 1990). Marquardt (2011) believed that internal and external stakeholders needed to be empowered to learn. Internal and external stakeholders were included in this research. A focus on empowerment required a paradigm shift in leadership. Leadership needed to shift from control to empowerment of employees, in the case of this research, empowerment of Midwest University's teacher candidates.

Marquardt (2011) further purported that people needed to be both "empowered and enabled to learn" (p. 26). Empowerment required that people had the "necessary resources at their disposal" (p. 26) while enabled meant that people had the knowledge to use those resources effectively. Marquardt's framework was useful in exploring the empowered and enabled learning of Midwest University's teacher candidates. Teacher candidates were provided with resources needed to gain program knowledge for educating the diverse group of learners who are $\mathrm{d} / \mathrm{DHH}$. The teacher candidates were simultaneously engaged in a variety of learning experiences to effectively integrate their knowledge into practice.

\section{Implications for Practice}

Although Midwest University's deaf education teacher preparation program resulted in deaf educators who were empowered and enabled to learn: (a) commitment to the profession, (b) proficiency in practice, and (c) to create learning impact in students who were d/DHH (MO-DESE, 2011, 2013), there were areas identified in which the program could improve. Therefore, it was important to consider how things might be done differently as a result of the research findings of this study. There were potential 
implications for practice within this deaf education teacher preparation program as well as within the state and nationally.

\section{Potential Changes within the Program}

Based on the findings, the number one area for program improvement, public school 101, the importance of navigating within the public school system, should be addressed within the program. The researcher is the off-site practicum supervisor. As a result of the findings, the researcher has begun to request deaf educators to present to the teacher candidates on specific topics during the teacher candidates' public school practicum seminar class. In light of the findings, the researcher plans to request that the invited speakers present on a topic focused on navigating within the public school system. When the researcher requests participation, public school 101 topics will be shared. This request may also include topic options for subject content areas and behavior and classroom management. The researcher plans to video-tape the presentations to build a repertoire of deaf educator expertise to share with subsequent teacher candidates without expecting deaf educators to return annually.

Prior to this study, during the fall 2013, the researcher adapted the off-site graduate practicum evaluation form (Appendix M) to better align with the new MODESE educator pillars (MO-DESE, 2011, 2013). It was at this stage that the researcher highlighted the directed focus on critical thinking, assessment and data analysis, positive risk-taking, and professional collaboration within the evaluation form. These items were double-weighted to emphasize their importance in word and in action, thus increasing the potential for impact on students' final grades in off-site practicum. Interestingly, the areas identified as needing more directed development in teacher candidates and 
implemented by the researcher during fall 2013, closely aligned with some of the positive emerging themes that surfaced within the data collected for this research.

The three emerging themes that specifically related to the previously mentioned off-site practicum targeted focus were: (a) developing critical thinking skills, (b) implementing evidence-based teaching, and (c) learning to collaborate. To enhance the alignment of this practicum experience with the findings of this study, the aforementioned emerging areas will continue to be targeted within the off-site practicum experience. In addition, a text book related to evidence-based teaching will be added to the course. Furthermore, modifications to the off-site practicum evaluation will be implemented in the fall 2015. One change will include an expansion of "attempts to take advantage of incidental learning opportunities" (Appendix M, p. 268) to include wording to emphasize the teaching skill, flexibility, for making on-the-spot modifications. Additionally, program faculty should discuss ways to improve integration of specific subject content and methodologies within coursework and practice.

\section{Potential Changes within the State}

Midwest University's deaf education preparation program resulted in deaf educators who were proficient in their practice. They were able to differentiate instruction to meet diverse learners who are $\mathrm{d} / \mathrm{DHH}$ needs. A notable strength of teacher candidates during their student teaching placements was their ability to communicate effectively in oral and manual modes of communication. In addition, overall, teacher candidates were very well prepared, flexible, knew how to teach, and had knowledge of coursework and experiences in practice. 
Coursework knowledge and practicum were intricately interwoven throughout graduate school at Midwest University. The program also front-loaded the practicum within the on-site preschool program for young children who are $\mathrm{d} / \mathrm{DHH}$. There were two areas considered for potential changes within the state: (a) practicum requirements, and (b) sign language requirements.

Practicum requirements. MO-DESE's state requirements indicated 75 hours of practicum was acceptable to meet certification requirements prior to student teaching. Midwest University teacher candidates completed many more hours. On average during the three years of this study, Midwest University teacher candidates completed 218 clock hours (ranging from 150-318.25 hours) prior to student teaching (Summary of Practicum and Student Teaching Clock Hours, 2014, Midwest University). This is 143 hours, approximately $96 \%$, more than the state required.

Midwest University also met national CED standards which required 150 practicum clock hours prior to student teaching (Summary of Practicum and Student Teaching Clock Hours, 2014, Midwest University). Program students accumulated 68 hours over (approximately $45 \%$ over) this national requirement. At Midwest University clock hours also included experiences beyond practicum (e.g., service-learning course experiences) with individuals who are $\mathrm{d} / \mathrm{DHH}$ under the supervision of program faculty.

Similarly, during the three year period of this study, all Midwest University student teachers exceeded the 250 minimum student teaching clock hours requirement of CED. Not including an outlier who completed significantly more hours, on average during the course of this study, graduate teacher candidates completed 383 hours at their student teaching placements. Given the high level of preparedness of Midwest 
University teacher candidates to teach the students who are $\mathrm{d} / \mathrm{DHH}$, perhaps the state may consider increasing practicum clock hour requirements to align with CED's national standard (CED, 2013). This increase in contact hours with students who are d/DHH may increase other teacher candidates' ability to meet the diverse learners' needs throughout the state.

Sign language requirements. MO-DESE state certification standards in the area of Deaf and Hard of Hearing, Birth to grade 12 required that teacher candidates meet a professional requirement for manual communications (MO-DESE, 2014). There is no specific criterion set for either the number of credit hours or the type of manual communication. Therefore, technically, having one university level course in sign language was sufficient to meet the state standard.

During the time of this study, Midwest University offered six different sign language courses, four in ASL and two in Conceptually Accurate Signed English (CASE). This deaf education teacher preparation program required teacher candidates who began the program as an undergraduate to complete four courses in sign language, two in ASL and two in CASE. At the graduate level, however, the program only required two courses and encouraged teacher candidates to take as many sign language courses as their schedules permitted.

During the three-year span of this study, there were a total of 17 program students. Eight of the teacher candidates, the accelerated masters' students were required to take four sign language courses (Summary of Program Students' Sign Language Courses Taken, 2014, Midwest University). The other nine teacher candidates were traditional master's option students. Those students entered the program at the graduate 
level so were only required to take 2 sign language courses (Summary of Program Students’ Sign Language Courses Taken, 2014, Midwest University).

Although nobody was required to do so, six (35.3\%) of the 17 program students took all six available sign language classes or for one of the teacher candidates tested out of one of the six courses so only took five (Summary of Program Students' Sign Language Courses Taken, 2014, Midwest University). Nine (52.9\%) of the teacher candidates completed four of the sign language courses. Of the remaining two students, one took three sign language courses but not all of the courses were taken at Midwest University, and one took the minimum two course requirement at the graduate level. Once again, Midwest University teacher candidates showed above and beyond commitment to the profession and dedication to learning to meet the needs of the diverse population of students who are d/DHH they served.

As previously stated, by completing one manual communication course, teacher candidates met the state requirement. The required state standard and MO-DESE's $(2011,2013)$ educational pillars deemed pivotal to the development of quality teachers may demonstrate a critical disconnect when it comes to addressing the communication needs of students who are d/Deaf or hard of hearing. Midwest University displayed commitment to meeting the needs of the diverse learners who are $\mathrm{d} / \mathrm{DHH}$ by providing a variety of sign language courses to teacher candidates. Furthermore, Midwest University required all students who began the program at the undergraduate level to take four sign language courses. Two sign language classes were required of teacher candidates entering the program at the graduate level. Every program student at least doubled the state's requirement in manual communication, with most surpassing this requirement by 
$400 \%$. In addition, almost all teacher candidates exceeded the program's requirements in the area of sign language.

Five different states were represented within this study: (a) two Midwestern states, (b) two southern states, and (c) one western state. Within those five states, $75 \%$ of the schools represented in this study included students who were $\mathrm{d} / \mathrm{DHH}$ who represented two or three different primary modes of communications. At the time of this study, U.S. public schools had a critical shortage of teachers of students who were d/DHH. And if the deaf education teacher preparation programs at risk of closing closed it would be most detrimental to the Midwest. Primary modes of communication should be an essential consideration for meeting the diverse learning needs of students who are d/DHH in U.S. public schools.

By increasing the state requirement in manual communication to two courses, the state may move a little closer to meeting the diverse communication needs of students who are $\mathrm{d} / \mathrm{DHH}$ in schools throughout the state. However, the state requirements would continue to lag behind the number of sign language courses that most Midwest University teacher candidates took. It may prove more beneficial to continue to support this comprehensive deaf education teacher preparation program's graduate students with partial tuition reimbursement scholarships. These scholarships may help recruit teacher candidates who fill the void for teachers of students who are $\mathrm{d} / \mathrm{DHH}$, teachers who are able to meet a variety of their students' modes of communication needs. In addition, the scholarships may simultaneously help sustain the only comprehensive deaf education teacher preparation program within the state by increasing enrollment at this university while the university continues to meet a community need through its on-site preschool. 


\section{Potential Changes within the Nation}

It was previously established that if deaf education teacher preparation programs at-risk of closing indeed close, the Midwest would be hit the hardest (Benedict et al., 2011). With program sustainability challenges (Benedict et al., 2011) and the need for teachers of students who are d/DHH in U.S. public schools (American Association for Employment in Education, 2008; U.S. Department of Education, 2012), there may need to be more national funding opportunities to support model deaf education teacher preparation programs who prepare teachers of students who are $\mathrm{d} / \mathrm{DHH}$ while simultaneously filling a community need by serving young children who are d/DHH and their families within their program walls.

Midwest University should pursue federal funding to help close the gap within U.S. schools for teachers of students who are d/DHH. Furthermore, funding could enhance Midwest teacher candidate preparation through a reinstitution of the parentinfant or early intervention ( $\mathrm{B}-3$ years) program which would also enhance service delivery to this population within the Midwest. Furthermore, this research demonstrated that Midwest University's espoused comprehensive philosophy was practiced and that teacher candidates were prepared to teach students who were $\mathrm{d} / \mathrm{DHH}$ regardless of students' primary mode of communication. This deaf education teacher preparation program has a responsibility to help meet the diverse learning needs of the population of students who are d/DHH within U.S. schools. Nationally, consideration for education funding should be given to deaf education teacher preparation programs who have demonstrated results and are able to meet the needs of the diverse population of students who are d/DHH. 


\section{Limitations}

The first limitation was that the researcher was a clinical professor at the program of study. The researcher took extra care to ascertain that the participants understood the voluntary aspect of their participation. In addition, because the researcher was faculty in the program, there was a possibility that the participants may have chosen to not be as forthcoming in their responses, instead, saying what they believed the researcher wanted to hear. The researcher believed the most vulnerable group for that limitation would be the current teacher candidates. All 1st and 2nd year teacher candidates as well as former program students, the seven deaf educators, received a survey that was returned to the researcher anonymously through Qualtrics.

Another limitation of this study was that the research was completed on one case. One case of a comprehensive deaf education teacher preparation program may be of significance to other comprehensive deaf education teacher preparation programs. The researcher provided detailed descriptions of the setting, research participants, and the students who were $\mathrm{d} / \mathrm{DHH}$ they served to better support transferability of the findings.

The researcher also found a few limitations within the procedures used to collect data. The researcher believed it was important for interview participants to have the option to member check their transcript. Out of the 11 preceptors interviewed, 10 chose to member check. The transcripts had been meticulously transcribed which then included word repetitions, false starts, and incomplete sentences that were said. Most participants who followed through with the member checking process made some comment about the difficulty they had reading what they said in a word for word narrative. They picked up on errors made which seemed to make the preceptors somewhat uncomfortable. The 
researcher had a similar response when reading mistakes and false starts that she had made within the interview process. In future research, omitting false starts and word repetitions may help alleviate some of the stress of reading transcripts.

A few other data collection challenges appeared within the survey. The researcher used the features on Qualtrics to allow the participants to save and continue at a later time; however, the researcher did not add the back button feature. The researcher had consciously made that decision because the researcher wanted the participants to answer one specific qualitative question without any prior knowledge of the MO-DESE framework questions that would follow.

The aforementioned question asked how the participant would describe a teacher candidate who was ready to teach students who are $\mathrm{d} /$ Deaf or hard of hearing at the end of student teaching. It was a philosophical question rather than a specific question about a particular teacher candidate. In retrospect, the researcher would consider adding the back button. Although this would allow the participants to see the questions that followed, the researcher missed some data when a small number of people read ahead and were unable to complete the questions unless they contacted the researcher to resend the survey.

Another potential limitation was the time the researcher listed in the consent form for completing the survey. The researcher said that it may take a possible 1 to $11 / 2$ hours to complete the survey. Although this could have been a deterrent, the high percentage of survey completion seemed to, again, indicate the commitment of the participants to this deaf education program, deaf education in general, or even perhaps, their connectedness to the researcher. 
The last survey challenge that the researcher noted was that the researcher had to go back into the survey and enlarge the text boxes in order to be able to print all the content that some of the participants included in their responses. Once the researcher enlarged the text boxes to do the printing, those who had not yet taken the survey would see the larger text boxes. The researcher was concerned that this may have indicated to the participants that they needed to fill in all the space or that it may have deterred them from completing the survey. The researcher mentioned within the reminder completion email that the larger text box size was only for printing purposes and did not indicate how much should be typed in response to the questions.

The final data collection challenge was the low return rate for the student teaching final evaluations and teaching philosophy statements. The researcher originally intended to receive access through program documents. It may have been beneficial to pursue that angle even if it required more steps in the IRB process. However, given the high return rate in every other area, the researcher believed this data would not have altered the study outcome. The researcher believed the additional documents would have simply increased confirmation of the findings as the few forms received had done.

\section{Future Research}

Future research could focus on expanding the learning impact portion of this study. Only emerging themes emerged within the research in learning impact, with the possible exception of meeting diverse learners' needs. Perhaps expanding this research to include district personnel who hired program graduates would produce additional evidence of learning impact. In addition, accessing the learning of the students who are $\mathrm{d} / \mathrm{DHH}$ that the former program graduates served could possibly be expanded. The later 
future research would be a much more arduous process due to a variety of factors such as IRB approval and parent permission.

With the increase in the number of students who are receiving cochlear implants and using spoken language, the researcher believes Midwest University could expand assessing the young children who are $\mathrm{d} / \mathrm{DHH}$ that attended the preschool or Midwest University's former parent-infant program. Assessment on the current or former students who are $\mathrm{d} / \mathrm{DHH}$ could be focused on collecting additional data to determine program effectiveness in learning impact specifically within the area of listening and spoken language. Perhaps studying some program 'firsts' such as the first program preschooler who received a cochlear implant, the first parent-infant child to receive a cochlear implant, the first child served with cochlear implant with additional disabilities, could be considered.

Within this deaf education teacher preparation program, most teacher candidates were reported to meet the diverse needs of students who were $\mathrm{d} / \mathrm{DHH}$, including mode of communication. This current study also revealed that schools serving students who were d/DHH during the data collection phase of this study, public schools and a School for the Deaf, tended to serve students who used different primary modes of communications. Most of the schools included students who were d/DHH who used at least two, if not all three modes of communication reported (spoken language, sign language with spoken language, and ASL).

The previously mentioned diversity of primary communication modes of students who are d/DHH may have ramifications for future research and future directions in hiring within the public school system. With the national shortage of teachers of students who 
are d/DHH within U.S. public schools, perhaps when a school district only needs one deaf educator, there should be special consideration to a teacher who is able to address the learning needs of this diverse population, beginning with modes of communication. This may be an efficient and effective way to help fill this shortage of deaf educators in the schools while meeting the diverse communication needs of students who are d/DHH.

Future research is also available within existing data collected during this study. The researcher should explore the responses of participants to a question asked prior to sharing MO-DESE's educator pillars on which the first three research questions were based. Each participant was asked what the ideal teacher candidate should 'look like' upon completion of student teaching. This data could be compared with the current study findings about the program's influence on empowering and enabling the learning of teacher candidates. Exploring the ideal deaf educator in relation to this deaf education teacher preparation program could be relevant to understanding this phenomenon of study.

Lastly, this researcher or other researchers could further explore a couple of additional findings that surfaced in chapter four. Specifically, the role of classroom and behavior management in teaching and exploring below the surface in teaching beyond academics could be considered. Teacher candidates' preparedness to teach was a dominate theme that surfaced within the research. Preparedness would seemingly be unlikely to surface if teacher candidates were ineffective in managing students' behaviors in the classroom. As a result of this study, the area of classroom and behavior management may warrant further exploration; however, it is not as compelling to the researcher as teaching beyond academics. 
The beyond academics emerging theme caught this researcher's attention. Participants who commented on the importance of teaching beyond academics emphasized aspects such as (a) developing cultural awareness of d/Deafness with students who were $\mathrm{d} / \mathrm{DHH}$, (b) the importance of connecting students who were $\mathrm{d} / \mathrm{DHH}$ with others who were d/DHH, and (c) fitting in socially and independently in a hearing world. This theme was the only theme within all of the research that was more heavily weighted in proportion to participants who were $\mathrm{d} / \mathrm{DHH}$ than participants who were hearing. The reason for this discrepancy in the voices heard may simply be that it did not happen to be voiced by the participants who were hearing, yet, the participants who were hearing also believed in the importance of teaching beyond academics. From the researcher's perspective, further exploration may be warranted.

Midwest University's deaf education teacher preparation program has resulted in deaf educators who were empowered and enabled to learn: (a) commitment to the profession, (b) proficiency in practice, and (c) to create learning impact in students who were $d$ /DHH (MO-DESE, 2011, 2013). The two most prevalent themes that were echoed from the voices of the participants were (a) diversity of d/Deafness and (b) differentiated instruction. Both of these themes spoke to the program's espoused comprehensive program materializing in the teacher candidates' practice. Most teacher candidates were prepared to teach students who were d/DHH regardless of the students' mode of communication. 


\section{References}

Alexiades, G., Asuncion, M., Hoffman, R. A., Kooper, R., Madell, J. R., Markoff, L. B., ...Sislian, N. (2008). Cochlear implants for infants and children. In B. Brandenburg \& I. Ip (Eds.), Pediatric audiology: Diagnosis, technology, and management (pp. 183-191). New York, NY: Thieme Medical Publishers.

Ambrose, S. E., Fey, M. E., \& Eisenberg, L. S. (2012). Phonological awareness and print knowledge of preschool children with cochlear implants. Journal of Speech, Language, and Hearing Research, 55, 811-823. doi:10.1044/10924388(2011/11-0086)

American Association for Employment in Education. (2008). Executive summary: Educator supply and demand in the United States. Columbus, OH: American Association for Employment in Education.

Anderson, K., \& Matkin, N. (1991). Relationship of long term hearing loss to psychosocial impact and educational needs, revised 2007. Retrieved from http://successforkidswithhearingloss.com/resources-for-professionals

Andrews, J. F., \& Covell, J. A. (2006/2007). Preparing future teachers and doctoral level leaders in deaf education: Meeting the challenge. American Annals of the Deaf. 151(5), 464-475.

Arnos, K. S., \& Pandya, A. (2007). Genetic causes of deafness. In S. Schwartz (Ed.), Choices in deafness: A parent's guide to communication options (3rd ed., pp. 7996). Bethesda, MD: Woodbine House.

Ausbrooks, M., Baker, S., \& Daugaard, J. (2012). Recruiting deaf and diverse teachers: Priorities of prescervice teachers in deaf education. Journal of the American Deafness \& Rehabilitation Association, 46(1), 369-398. 
Beer, J., Harris, M., Kronenberger, W. G., Holt, R. F., \& Pisoni, D. B. (2012). Auditory skills, language development, and adaptive behavior of children with cochlear implants and additional disabilities. International Journal of Audiology, 51, $495-$ 498. doi:10.3109/14992027.2012.664291

Belasen, A. T. (2000). Leading the learning organization. Albany, NY: State University of New York Press.

Benedict, B. S., \& Sass-Lehrer, M. (2007). The ASL and English bilingual approach: A professional perspective. In S. Schwartz (Ed.), Choices in deafness: A parents' guide to communication options (3rd ed, pp. 185-204). Bethesda, MD: Woodbine House.

Benedict, K. M., Johnson, H., \& Antia, S. D. (2011). Faculty needs, doctoral preparation, and the future of teacher preparation programs in the education of deaf and hard of hearing students. American Annals of the Deaf, 156(1), 35-46.

Bess, F. H., \& Tharpe, A. M. (1984). Unilateral hearing impairment in children. Pediatrics, 74(2), 206-216.

Bolman, L. G., \& Deal, T. E. (2008). Reframing organizations: Artistry, choice, and leadership (4th ed.). San Francisco, CA: Jossey-Bass.

Bruce, S., DiNatale, P., \& Ford, J. (2008). Meeting the needs of deaf and hard of hearing students with additional disabilities through professional teacher development. American Annals of the Deaf, 153(4), 368-375.

Centers for Disease Control and Prevention. (2011). Summary of 2011 national CDC EHDI data. Retreived from http://www.cdc.gov/ncbddd/hearingloss/2011data/2011_ehdi_hsfs_summary_a.pdf 
Cole, E. B., \& Flexer, C. (2011). Children with hearing loss: Developing listening and talking birth to six (2nd. ed.). San Diego, CA: Plural Publishing.

Compton, M. V., Niemeyer, J. A., \& Michael, S. (2004). Auditory/Oral birthkindergarten teacher preparation: A research based model. The Volta Review, 104(2), 93-106.

Council on Education of the Deaf (CED), Office of Program Accreditation. (2013).

Manual 1: CED standards for programs preparing teachers of students who are deaf and hard of hearing. Retrieved from http://councilondeafed.org/ CEDManual1Rev5_13.pdf

Council on Education of the Deaf (CED), Office of Program Evaluation. (2013). Accreditation. Manual 2 accreditation process: What you need to do. Retrieved from http://councilondeafed.org/accreditation.html

Crittenden, J. B. (1993). The culture and identity of deafness. In P. Paul \& D. Jackson, Toward a psychology of deafness: Theoretical and empirical perspectives (pp. 215-236). Needham Heights, MA: Allyn \& Bacon.

Creswell, J. (2008). Educational research: Planning, conducting, and evaluating quantitative and qualitative research (3rd ed.). Upper Saddle River, NJ: Pearson Education.

Creswell, J. W. (2009). Research design: Qualitative, quantitative, and mixed methods approaches (3rd. ed.). Thousand Oaks, CA: Sage.

Creswell, J. (2012). Educational research: Planning, conducting, and evaluating quantitative and qualitative research (4th ed.). Boston, MA: Pearson Education. 
Deaf Education Teacher Preparation Programs. (2012). Deaf education: Educational enhancement for the field of deaf education. Retrieved from http://www.deafed.net/PageText.asp?hdnPageId=120

Denzin, N. K., \& Lincoln, Y. S. (Eds.). (2005). The sage handbook of qualitative research (3rd ed.). Thousand Oaks, CA: Sage.

Dodd, E. E., \& Scheetz, N. A. (2003). Preparing today's teachers of the deaf and hard of hearing to work with tomorrow's students: A statewide needs assessment. American Annals of the Deaf, 148(1), 25-30.

Dolman, D. (2008). College and university requirements for teachers of the deaf at the undergraduate level: A twenty-year comparison. American Annals of the Deaf, 153(3), 322-327.

Dolman, D. (2010). Enrollment trends in deaf education teacher preparation programs, 1973-2009. American Annals of the Deaf, 155(3), 353-359.

Eisner, E. W. (1991). The enlightened eye: Qualitative inquiry and the enhancement of educational practice. New York, NY: Macmillian.

Emerson, R., Fretz, R., \& Shaw, L. (1995). Writing ethnographic fieldnotes (2nd ed.). Chicago, IL: University of Chicago Press.

Fink, A. (2009). How to conduct surveys: A step-by-step guide (4th ed.). Thousand Oaks, CA: Sage.

Flexer, C. (1994). Facilitating hearing and listening in young children. San Diego, CA: Singular.

Flexer, C., \& Madell, J. R. (2008). Why is hearing important in children? In B. Brandenburg \& I. Ip (Eds.), Pediatric audiology: Diagnosis, technology, and management (pp. xix-xxii). New York, NY: Thieme Medical. 
Fraenkel, J. R., \& Wallen, N. E. (2009). How to design and evaluate research in education. NewYork, NY: The McGraw-Hill Companies.

Gallaudet Research Institute. (2011). Regional and national summary report of data from the 2009-10 annual survey of deaf and hard of hearing children and youth. Washington, DC: GRI, Gallaudet University.

Gay, L. R., Mills, G. E., \& Airasian, P. (2012). Educational research: Competencies for Analysis and applications (10th ed.). Boston, MA: Pearson Education.

Geers, A. E., \& Nicholas, J. G. (2013). Enduring advantages of early cochlear implantation for spoken language development. Journal of Speech, Language, and Hearing Research, 56, 643-653.

Grant, G., \& Murray, C. E. (2002). Teaching in America: The slow revolution. Cambridge, MA: First Harvard University Press.

Hatch, J. A. (2002). Doing qualitative research in education settings. Albany, NY: SUNY Press.

Hayes, H. (2010). Washington University School of Medicine: A distinctive program in deaf education studies at the program in audiology and communication sciences (PACS). The Volta Review, 110(2), 271-278.

Holden-Pitt, L., \& Diaz, J. (1998). Thirty years of the annual survey of deaf and hard-ofhearing children \& youth: A glance over the decades. American Annals of the Deaf, 143(2), 72-76.

Houston, T. K. (2010). Utah State University: Cross-discipline training through the graduate studies program in auditory learning \& spoken language. The Volta Review, 110(2), 323-330. 
Humphries, T., \& Allen, B. (2008). Reorganizing teacher preparation in deaf education. Sign Language Studies, 8(2), 160-180.

Johnson, H. A. (2004). U.S. deaf education teacher preparation programs: A look at the present and a vision for the future. American Annals of the Deaf, 149(2), 75-91.

Johnson, H. A. (2013). Initial and ongoing teacher preparation and support: Current problems and possible solutions. American Annals of the Deaf, 157(5), 439-449.

Joint Committee on Infant Hearing Loss. (2007). Supplement to the JCIH 2007 position statement: Principles and guidelines for early intervention after confirmation that a child is deaf or hard of hearing. The Volta Review, 113(2), 171-225.

Jones, T., \& Jones, J. (2003). Educating young deaf children with multiple disabilities. In B. Bodner-Johnson \& M. Sass-Lehrer (Eds.), The young deaf and hard-of-hearing child (pp. 297-332). Baltimore, MD: Paul H. Brookes.

King, C. M, \& Quigley, S. P. (1985). Reading and deafness. San Diego, CA: CollegeHill.

Kirk, K. I., Miyamoto, R. T., Ying, E. A., Perdew, A. E., \& Zuganelis, H. (2002). Cochlear implantation in young children: Effects of age at implantation and communication mode. Volta Review, 102(4), 127-144.

Kouzes, J. M., \& Posner, B. Z. (2010). The truth about leadership: The no-fads, heart-ofthe-matter facts you need to know. San Fracisco, CA: Jossey-Bass.

Kouzes, J. M., \& Posner, B. Z. (2011). The five practices of exemplary leadership. San Francisco, CA: Wiley.

LaSasso, C., \& Wilson, A. (2000). Results of two national surveys of leadership personnel needs in deaf education. American Annals of the Deaf, 145(5), 429-435. 
Lederberg, A. R., Schick, B., \& Spencer, P. E. (2012). Language and literacy development of deaf and hard-of-hearing children: Successes and challenges. Developmental Psychology, 49(1), 15-30. doi:10.1037/a0029558

Lenihan, S. (2010). Trends and challenges in teacher preparation in deaf education. The Volta Review, 110(2), 117-128.

Lincoln, Y. S., \& Guba, E. G. (1985). Naturalistic inquiry. Thousand Oaks, CA: Sage. Ling, D. (1989). Foundations of spoken language for hearing-impaired children. Washington, D.C.: Alexander Graham Bell Association.

Luckner, J. L. (2013). Using the dynamic indicators of basic early literacy skills with students who are deaf or hard of hearing: Perspectives of a panel of experts. American Annals of the Deaf, 158(1), 7-19.

Luckner, J. L., \& Howell, J. (2002). Suggestions for preparing itinerant teachers: A qualitative analysis. American Annals of the Deaf, 147(3), 54-61.

Luckner, J. L., \& Muir, S. (2001). Successful students who are deaf in general education settings. American Annals of the Deaf, 146(5), 435-446. doi:10.1353/aad 2012. 0202

Luckner, J. L., Sebald, A. M., Cooney, J., Young III, J., \& Muir, S. G. (2005/2006). An examination of the evidence-based literacy research in deaf education. American Annals of the Deaf, 150(5), 443-456.

Madell, J. R., \& Flexer, C. (2008). Pediatric audiology: Diagnosis, technology, and management. New York, NY: Thieme Medical Publishers. 
Marquardt, M. J. (2011). Building the learning organization: Achieving strategic advantage through a commitment to learning (3rd ed.). Boston, MA: Nicholas Brealey.

Marschark, M., Lang, H. G., \& Albertini, J. A. (2002). Educating deaf students: From research to practice. New York, NY: Oxford.

Marvelli, A. L. (2010). Highlights in the history of oral teacher preparation in America. The Volta Review, 110(2), 89-115.

Maslow, A. H. (2011). A theory of human motivation. In J. M. Shafritz, J. S. Ott, \& Y. S. Jang (Eds.), Classics of organization theory $\left(7^{\text {th }}\right.$ ed, pp. 171-182). Boston, MA: Nicholas Brealey.

McGinnis, M. (2010). John Tracy Clinic/University of San Diego graduate program: A distance learning model. The Volta Review, 110(2), 261-270.

McGregor, D. M. (2011). The human side of enterprise. In J. M. Shafritz, J. S. Ott, \& Y. S. Jang (Eds.), Classics of organization theory (7th ed, pp. 183-188). Boston, MA: Nicholas Brealey.

Meinzen-Derr, J., Wiley, S., \& Choo, D. I. (2011). Impact of early intervention on expressive and receptive language development among young children with permanent hearing loss. American Annals of the Deaf, 155(5), 580-591.

Merriam, S. (2009). Qualitative research: A guide to design and implementation (2nd ed.). San Franciso, CA: Jossey-Bass.

Mertens, D. M. (2015). Research and evaluation in education and psychology. (4th ed.). Thousand Oaks, CA: Sage. 
Michigan State University College of Education. (n.d.). Deaf education programs. Retreived from http://education.msu.edu/deafed/

Missouri Department of Elementary and Secondary Education. (2011). Missouri model teacher and leader standards: A resource for state dialogue. Retrieved from http://dese.mo.gov/eq/documents/StandardsInformationDocument.pdf

Missouri Department of Elementary and Secondary Education. (2013). Teacher standards: Missouri's educator evaluation system. Retrieved from http://dese .mo.gov/eq/documents/00-TeacherEvaluation-CompleteDoc.pdf

Mitchell, R. E., \& Karchmer, M. A. (2004). Chasing the mythical ten percent: Parental hearing status of deaf and hard of hearing students in the united states. Sign Language Studies, 4(2), 138-163.

Mitchell, R. E., \& Karchmer, M. A. (2006). Demographics of deaf education: More students in more places. American Annals of the Deaf, 151(2), 95-104.

Moog, J. S. (2007). The auditory-oral approach: A professional perspective. In S. Schwartz (Ed.), Choices in deafness: A parents' guide to communication options (3rd ed, pp. 131-145). Bethesda, MD: Woodbine House.

Moores, D. F. (2001). Educating the deaf: Psychology, principles, and practices (5th ed.). Boston, MA: Houghton Mifflin.

Most, T., \& Tsach, N. (2010). School functioning of children with unilateral hearing loss in comparison to the functioning of children with normal hearing. Journal of the American Deafness \& Rehabilitation Association, 43(2), 101-119.

National Center for Education Statistics. (2006). School locale definitions. Retrieved from http://nces.ed.gov/surveys/ruraled/definitions.asp 
National Center for Education Statistics. (2012). Search for public schools. Retrieved from http://nces.ed.gov/ccd/districtsearch/index.asp?ID2=1705570

National Center for Hearing Assessment and Management. (2011). Background of the national center for hearing assessment \& management. Retrieved from http://www.infanthearing.org/background/index.html

National Institute on Deafness and Other Communication Disorders (NIDCD). (2010). Quick statistics. Retrieved from www.nidcd.nih.gov/health/statistics /Pages/quick.aspx

Nicholas, J. G., \& Geers, A. E. (2008). Expected test scores for preschoolers with a cochlear implant who use spoken language. American Journal of SpeechLanguage Pathology, 17, 121-138. doi:1058-0360/08/1702-0121

Northern, J. L., \& Downs, M. P. (1991). Hearing in children (4th ed.). Baltimore, MD: Williams \& Wilkins.

Paterson, M. M., \& Cole, E. (2010). The university of Hartford and CREC Soundbridge: A new master's of education in aural habilitation and education of hearing impaired children. The Volta Review, 110(2), 279-291.

Patton, M. Q. (1999). Enhancing the quality and credibility of qualitative analysis. Health Services Research, 34(5), 1189-1208.

Paul, P. V., \& Jackson, D. W. (1993). Toward a psychology of deafness: Theoretical and empirical perspectives. Needham Heights, MA: Allyn \& Bacon.

Paul, P. V., Wang, Y., Trezek, B. J., \& Luckner, J. L. (2009). Phonology is necessary, but not sufficient: A rejoinder. American Annals of the Deaf, 154(4), 346-356. 
Paul, P. V., \& Whitelaw, G. M. (2011). Hearing and deafness: An introduction for health and education professionals. Sudbury, MA: Jones and Bartlett.

Qualtrics. (2013). Online Survey Software (Version 37,892) [Software]. Provo, Utah: Qualtrics. Retreived from http://qualtrics.com/

Rice, C. J., \& Goessling, D. P. (2005). Recruiting and retaining male special education teachers. Remidial and Special Education, 20(6), 347-356.

Savin-Baden, M., \& Major, C. H. (2013). Qualitative research: The essential guide to theory and practice. Abingdon, Oxon: Routledge.

Scheetz, N. A. (2001). Orientation to deafness. Boston, MA: Allyn and Bacon.

Schein, E. H. (2010). Organizational culture and leadership (4th ed.). San Francisco, CA: Jossey-Bass.

Schirmer, B. R. (2001). Psychological, social, and educational dimensions of deafness. Needham Heights, MA: Allyn and Bacon.

Schow, R. L., \& Nerbonne, M. A. (2002). Introduction to audiologic rehabilitation $\left(4^{\text {th }}\right.$ ed.). Boston, MA: Allyn \& Bacon.

Senge, P. M. (2006). The fifth discipline: The art and practice of the learning organization. New York, NY: Doubleday.

Senge, P. M. (1990). The fifth discipline: The art and practice of the learning organization. New York, NY: Doubleday.

Senge, P. M., Roberts, C., Ross, R. B., Smith, B. J., \& Kleiner, A. (1994). The fifth discipline fieldbook: Strategies and tools for building a learning organization. New York, NY: Doubleday. 
Simms, L., Rusher, M., Andrews, J. F., \& Coryell, J. (2008). Apartheid in deaf education: Examining workforce diversity. American Annals of the Deaf, 153(4), 384-395.

Smith, J. (2007). Cued speech: A professional perspective. In Schwartz, S. (Ed.), Choices in deafness: A parent's guide to communication options (3rd ed, pp. 223-256). Bethesda, MD: Woodbine House.

Spencer, P. E., \& Marschark, M. (2010). Evidence-based practice in educating deaf and hard-of-hearing students. New York, NY: Oxford.

Stake, R. E. (2005). Qualitative case studies. In N. K. Denzin \& Y. S. Lincoln (Eds.), The Sage handbook of qualitative research (3rd ed, pp. 443-466). Thousand Oaks, CA: Sage.

Stenross, B. (1999). Missed connections: Hard of hearing in a hearing world. Philadelphia, PA: Temple University.

Syverud, S. M., Guardino, C., \& Selznick, D. N. (2009). Teaching phonological skills to a deaf first grader: A promising strategy. American Annals of the Deaf, 154(4), 382-388.

Teller, H., \& Harney, J. (2005/2006). Views from the field: Program directors' perceptions of teacher education and the education of students who are deaf or hard of hearing. American Annals of the Deaf, 150(5), 470-479.

The National Agenda. (2005). Moving forward on achieving educational equality for deaf and hard of hearing students. Austin, TX: Texas School for the Deaf.

Traxler, C. B. (2000). The Stanford Achievement Test, $9^{\text {th }}$ Edition: National norming and performance standards for deaf and hard-of-hearing students. Journal of Deaf Studies and Deaf Education, 5(4), 337-348. doi:10.1093/deafed/5.4.337 
Trezek, B. J., Wang, Y., \& Paul, P. V. (2010). Reading and deafness: Theory, research, and practice. Clifton Park, NY: Delmar.

Tye-Murray, N. (2009). Foundations of aural rehabilitation: Children, adults, and their family members (3rd ed.). Clifton Park, NY: Delmar.

U.S. Department of Education, National Center for Education Statistics. (2012). The condition of education. Retrieved from http://nces.ed.gov/pubs2012/2012045_5.pdf

U.S. Department of Education, Office of Special Education and Rehabilitative Services, \& Office of Special Education Programs. (2012). 31st annual report to the congress on the implementation of the individuals with disabilities education act, 2009. Washington, DC: U.S. Department of Education.

Waddy-Smith, B., \& Wilson, V. (2005). See the sound: Visual phonics. Overland Park, KS: Gallaudet University Regional Center.

Wang, Y., \& Engler, K. S. (2011). Early intervention. In P. Paul \& G. Whitelaw (Eds.), Hearing and deafness: An introduction for health and education professionals (pp. 241-269). Sudbury, MA: Jones and Bartlett.

Wang, Y., Engler, K., \& Oetting, T. (2014). Expectations lead to performance: The transformative power of high expectations in pre-school. Odyssey, 15, 36-39.

Wang, Y., Spychala, H., Harris, R. S., \& Oetting, T. L. (2013). The effectiveness of a phonics-based early intervention for deaf and hard of hearing preschool children and its possible impact on reading skills in elementary schools: A case study. American Annals of the Deaf, 158(2), 107-120. 
Wang, Y., Trezek, B. J., Luckner, J. L., \& Paul, P. V. (2008). The role of phonology and phonologically related skills in reading instruction for students who are deaf or hard of hearing. American Annals of the Deaf, 153(4), 396-407.

Welling, D. R., \& Ukstins, C. A. (2015). Fundamentals of audiology for the speechlanguage pathologist. Burlington, MA: Jones and Bartlett.

White, K. R. (2008). Newborn hearing screening. In B. Brandenburg \& I. Ip (Eds.), Pediatric audiology: Diagnosis, technology, and management (pp. 183-191). New York, NY: Thieme Medical Publishers.

Winn, S. L. (2007). Employment outcomes for the congenitally deaf in Australia: Has anything changed? American Annals of the Deaf, 152(1), 382-390.

Wolcott, H. F. (1995). The art of fieldwork. Walnut Creek, CA: AltaMira Press.

Yoshinaga-Itano, C. (2003). Early intervention after universal neonatal hearing screening: Impact on outcomes. Mental Retardation and Developmental Disabilities Research Reviews, 9, 252-266. 


\section{Appendix A}

\section{Preceptor Interview Consent to Participate in Research}

Project Title: A Case Study of a Comprehensive Graduate Deaf Education Teacher Preparation Program: Implications for Collaborative Educational Leadership in Diverse Contexts

Researcher: Karen S. Engler, doctoral student, University of Missouri

Faculty Sponsor: Dr. Cynthia MacGregor, professor, MSU Counseling Leadership \& Special Education

\section{Introduction:}

You are being asked to take part in a phenomenological case study being conducted by me, Karen Engler, for my dissertation under the direction of advisor, Dr. Cynthia MacGregor, professor in the Department of Counseling Leadership \& Special Education at Missouri State University and University of Missouri - Missouri State University Site Coordinator of the Educational Leadership and Policy Analysis EdD program.

It is at this time, if you choose, that you will sign the consent form to indicate your willingness to participate. Your consent form will be stored in a separate folder from your interview protocol to protect your privacy. All data will be stored in a locked file cabinet in the researcher's office.

\section{Purpose:}

The purpose of this research study is to describe a Midwest's comprehensive, graduate deaf education teacher preparation program and its people through a detailed exploration of empowered and enabled learning of teacher candidates (Marquardt, 2011). Your lived experiences and perspective are believed to be important for understanding this phenomenon.

\section{Request for Voluntary Participation:}

You are being invited to participate in an interview for this case study. It is up to you whether you would like to participate. If you decide not to participate, there is no penalty. If you decide to participate, you are free to decide to stop at any time during the interview process. You may decide to not answer any question that you do not want to answer. You may even decide to withdraw your interview data after you have participated in the interview with no penalty.

\section{Procedures:}

The interview will take approximately one hour. During the interview, you will be asked questions about your experiences with this deaf education teacher preparation program and its graduate teacher candidates. You will also be asked to respond to 12 demographic types of questions at the end of the interview. Responses to the demographic items and your answer to qualitative question one will allow the researcher to describe the pool of research participants and the students who are $\mathrm{d} / \mathrm{DHH}$ that they serve. 
During the interview, you will have the opportunity to share information that you would like to add that did not come out through the questions asked. One question that you will be asked is, from your perspective, at the end of student teaching, what does a teacher candidate "look like" who is ready to learn to teach students who are d/DHH?

The interview will be audio-taped and transcribed. You will be given the opportunity to member check to make sure that the transcribed document is what you intended to say. The interview results may be used as part of the dissertation, publications, and other scholarly work.

\section{Privacy:}

All of the information collected during the interview will be kept confidential. Your name will not be recorded on the interview forms or used within the dissertation to identify you.

\section{Explanation of Risks:}

The risks of this interview are similar to the risks of everyday life. You may experience discomfort if you feel anxious responding to questions in an interview format when being audio-taped.

\section{Explanation of Benefits:}

There will no direct benefit to you for participating in this study. Your willingness to share your experiences and knowledge will contribute to this dissertation and may result in benefit to others within the field of deaf education.

\section{Contacts and Questions:}

If you have any questions or want to voice concerns or complaints about this study, please contact dissertation advisor, Dr. Cynthia MacGregor or the researcher, Karen Engler. If you need an explanation of this document or require accommodations to complete the interview process, please contact the researcher or advisor. Dr. MacGregor may be reached at (417) 836-6046 or CMacgregor@MissouriState.edu . You may reach the researcher at (417) 836-66764 or KarenEngler@MissouriState.edu . If you have any questions regarding your rights as a human subject participant, you can contact the Campus Institutional Review Board at 573-882-9585 or email umcresearchcirb@missouri.edu.

I have read this form and agree to participate.

Signature:

Date:

Printed Name: 


\section{Appendix B}

Faculty Survey Consent to Participate in Research

Project Title: A Case Study of a Comprehensive Graduate Deaf Education Teacher Preparation Program: Implications for Collaborative Educational Leadership in Diverse Contexts

Researcher: Karen S. Engler, doctoral student, University of Missouri

Faculty Sponsor: Dr. Cynthia MacGregor, professor, MSU Counseling Leadership \& Special Education

\section{Introduction:}

You are being asked to take part in a phenomenological case study being conducted by me, Karen Engler, for my dissertation under the direction of advisor, Dr. Cynthia MacGregor, professor in the Department of Counseling Leadership \& Special Education at Missouri State University and University of Missouri - Missouri State University Site Coordinator of the Educational Leadership and Policy Analysis EdD program.

It is at this time, if you choose, that you will indicate your willingness to participate by clicking on the radio button. Your willingness to participate will also be fulfilled by you clicking on the survey and completing the survey. All survey data will be kept within a secured Qualtrics site. Printed survey data will be stored in a locked file cabinet in the researcher's office.

\section{Purpose:}

The purpose of this research study is to describe a Midwest's comprehensive, graduate deaf education teacher preparation program and its people through a detailed exploration of empowered and enabled learning of teacher candidates (Marquardt, 2011). Your lived experiences and perspective are believed to be important for understanding this phenomenon.

\section{Request for Voluntary Participation:}

You are being invited to participate in a survey for this case study. It is up to you whether you would like to participate. If you decide not to participate, there is no penalty. If you decide to participate, you are free to decide to stop at any time during the survey process. You may decide to not answer any question that you do not want to answer. You may even decide to withdraw your survey data after you have participated in the survey with no penalty by contacting the researcher or the researcher's advisor, Dr. Cynthia MacGregor.

\section{Procedures:}

The survey may take 1-1 1/2 hours to complete depending on how much time you spend on the eight qualitative questions. On the survey, you will be asked questions about your experiences with this deaf education teacher preparation program and its graduate teacher candidates. You will also be asked a few demographic items. Your responses to the demographic items and qualitative question one will allow the researcher to describe the pool of deaf education program faculty participants. 
The final qualitative question will provide you with the opportunity to share information that you would like to add that was not included in the surveyed items. One question that you will be asked is, from your perspective, at the end of student teaching, what does a teacher candidate "look like" who is ready to teach students who are d/DHH? The survey results may be used as part of the dissertation, publications, and other scholarly work.

\section{Privacy:}

All of the information collected during the survey will be kept confidential. Your name will not be used on the survey form or within the dissertation to identify you. However, with only two full-time deaf education teacher preparation faculty being surveyed it may be almost impossible for the researcher to be unaware of which faculty completed which survey. The group of five per course faculty may make it more difficult for the researcher to know who completed those surveys. If you are able to be identified through demographic data or through your responses to the questions, your identity will be kept confidential.

\section{Explanation of Risks:}

The risks of this survey are similar to the risks of everyday life. You may experience discomfort if you feel anxious responding to questions in a survey when you believe the person may know who completed the survey.

\section{Explanation of Benefits:}

There will no direct benefit to you for participating in this study. Your willingness to share your experiences and knowledge will contribute to this dissertation and may result in benefit to others within the field of deaf education.

\section{Contacts and Questions:}

If you have any questions or want to voice concerns or complaints about this study, please contact dissertation advisor, Dr. Cynthia MacGregor or the researcher, Karen Engler. If you need an explanation of this document or require accommodations to complete the survey, please contact the researcher or advisor. Dr. MacGregor may be reached at (417) 836-6046 or CMacgregor@MissouriState.edu . You may reach the researcher at (417) 836-66764 or KarenEngler@,MissouriState.edu . If you have any questions regarding your rights as a human subject participant, you can contact the Campus Institutional Review Board at 573-882-9585 or email umcresearchcirb@missouri.edu.

Respectfully,

Karen S. Engler

Doctoral Student

University of Missouri 


\section{Appendix C}

Student Survey Consent to Participate in Research

Project Title: A Case Study of a Comprehensive Graduate Deaf Education Teacher Preparation Program: Implications for Collaborative Educational Leadership in Diverse Contexts

Researcher: Karen S. Engler, doctoral student, University of Missouri

Faculty Sponsor: Dr. Cynthia MacGregor, professor, MSU Counseling Leadership \& Special Education

\section{Introduction:}

You are being asked to take part in a phenomenological case study being conducted by me, Karen Engler, for my dissertation under the direction of advisor, Dr. Cynthia MacGregor, professor in the Department of Counseling Leadership \& Special Education at Missouri State University and University of Missouri - Missouri State University Site Coordinator of the Educational Leadership and Policy Analysis EdD program.

It is at this time, if you choose, that you will indicate your willingness to participate by clicking on the radio button. Your willingness to participate will also be fulfilled by you clicking on the survey and completing the survey. All survey data will be kept within a secured Qualtrics site. Printed survey data will be stored in a locked file cabinet in the researcher's office. You are also being asked if you would supply the researcher with your Student Teaching Final Evaluation and your Teaching Philosophy Statement. Again, this is completely voluntary on your part. The data collected from these documents will be put on Program Document Protocol and will be deidentified, a document ID will be used in place of your name. Also, this data will not be linked to the survey that you will take.

\section{Purpose:}

The purpose of this research study is to describe a Midwest's comprehensive, graduate deaf education teacher preparation program and its people through a detailed exploration of empowered and enabled learning of teacher candidates (Marquardt, 2011). Your lived experiences and perspective are believed to be important for understanding this phenomenon.

\section{Request for Voluntary Participation:}

You are being invited to participate in a survey for this case study as well as to supply the researcher with your Student Teaching Final Evaluation and Teaching Philosophy Statement for those of you who have those archival documents. It is up to you whether you would like to participate in some or all of the aforementioned items. If you decide not to participate, there is no penalty. If you decide to participate, you are free to decide to stop at any time during the survey process. You may decide to not answer any question that you do not want to answer. You may even decide to withdraw your survey data after you have participated in the survey with no penalty by contacting the researcher or the researcher's advisor, Dr. Cynthia MacGregor. 


\section{Procedures:}

The survey may take 1-1 1/2 hours to complete depending on how much time you spend on the eight qualitative questions. On the survey, you will be asked questions about your experiences with this deaf education teacher preparation program as it relates to you, a teacher candidate or former teacher candidate of the program. You will also be asked to respond to 9 demographic types of questions at the end of the survey which are provided with a closed set of responses in multiple choice format. Your responses to the demographic items and qualitative question one will allow the researcher to describe the pool of research participants and the students who are $\mathrm{d} / \mathrm{DHH}$ that you serve.

The final qualitative question will provide you with the opportunity to share information that you would like to add that was not included in the surveyed items. One question that you will be asked is, from your perspective, at the end of student teaching, what does a teacher candidate "look like" who is ready to teach students who are d/DHH? The survey results may be used as part of the dissertation, publications, and other scholarly work.

\section{Privacy:}

All of the information collected during the survey will be kept confidential. Your name will not be used on the survey form or within the dissertation to identify you. However, with only two 2 nd year teacher candidates being invited to participate, it will be almost impossible for the researcher to be unaware of which 2nd year graduate student completed which survey. If you or others are able to be identified through demographic data or through your responses to the questions, your identity will be kept confidential.

\section{Explanation of Risks:}

The risks of this survey are similar to the risks of everyday life. You may experience discomfort if you feel anxious responding to questions in a survey when you believe the person may know who completed the survey.

\section{Explanation of Benefits:}

There will no direct benefit to you for participating in this study. Your willingness to share your experiences and knowledge will contribute to this dissertation and may result in benefit to others within the field of deaf education.

\section{Contacts and Questions:}

If you have any questions or want to voice concerns or complaints about this study, please contact dissertation advisor, Dr. Cynthia MacGregor or the researcher, Karen Engler. If you need an explanation of this document or require accommodations to complete the survey, please contact the researcher or advisor. Dr. MacGregor may be reached at (417) 836-6046 or CMacgregor@MissouriState.edu . You may reach the researcher at (417) 836-66764 or KarenEngler@,MissouriState.edu . If you have any questions regarding your rights as a human subject participant, you can contact the Campus Institutional Review Board at 573-882-9585 or email umcresearchcirb@missouri.edu.

Especially for current teacher candidates of this program, I want to be sure you understand that your participation is voluntary. It is completely your choice. There is no 
penalty for not participating. If you decide to participate, you may withdraw your participation at any time.

Respectfully,

Karen S. Engler

Doctoral Student

University of Missouri 


\section{Appendix D}

$$
\text { Preceptor Interview Protocol - Part One }
$$

Interviewee Category:

Off-site Practicum Preceptor

Student Teaching Preceptor
Interviewee ID:

Date Interviewed:

Qualitative Interview Questions: The following questions are designed to gain an understanding of Marquardt's (2011) empowered and enabled learning phenomenon as it relates to teacher candidates preparation to teach students who are $\mathrm{d} / \mathrm{DHH}$ through this deaf education teacher preparation program. Your lived experiences and perspective are important to understanding this studied phenomenon.

1. Tell me a little about yourself, your background, and what got you interested in teaching students who are d/DHH.

2. From your perspective, at the end of student teaching, what does a teacher candidate "look like" who is ready to teach students who are $\mathrm{d} / \mathrm{DHH}$ ?

3. In what ways, if any, does the program help teacher candidates learn commitment to the Deaf education profession?

4. In what ways, if any, does the program prepare teacher candidates to be proficient in practice?

5. In what ways, if any, does the program prepare teacher candidates to create learning impact in students who are $\mathrm{d} / \mathrm{DHH}$ ?

6. How could the program improve how teacher candidates are prepared to teach students who are d/DHH?

7. Please comment on the relationship between teacher candidates' hearing status and their teaching of your students who are $\mathrm{d} / \mathrm{DHH}$.

8. Is there anything that we haven't explored yet that would be helpful for me to know? 


\section{Appendix E}

$$
\text { Preceptor Interview Protocol - Part Two }
$$

Interviewee Category:

Off-site Practicum Preceptor

Student Teaching Preceptor
Interviewee ID:

Date Interviewed:

Demographic Interview Items:

The following items will provide the researcher with information to better describe the pool of research participants, the participants' schools, and the students who are d/DHH whom they serve. Each question is provided with a closed set of responses in multiple choice format. There are 12 questions in this section. This section should take no more than 5-10 minutes to complete.

1. Which of the following best describes your role with this deaf education teacher preparation program?

a. Off-site practicum preceptor and former program graduate

b. Off-site practicum preceptor and NOT a program graduate

c. Student teaching preceptor and former program graduate

d. Student teaching preceptor and NOT a program graduate

e. Off-site practicum and student teaching preceptor and program graduate

f. Off-site practicum and student teaching preceptor, program graduate, and have served as program per course faculty

2. Which category best indicates your professional years in the field of Deaf Education?
a. Less than 1 year
b. 1 - 2 years
c. 3-5 years
d. 6-10 years
e. 11-15 years
f. 16-20 years
g. More than 20 years

3. Which of the following best describes your school which serves individuals who are $\mathrm{d} / \mathrm{DHH}$ ?
a. Public school district
b. Private school
c. School for the Deaf 
4. Which of the following best describes your current role in your school?
a. Administrator
b. Deaf educator
c. Other: Please indicate role:

5. Which of the following indicates the number of additional deaf educators in your school (not counting yourself)?
a. 0
b. 1
c. 2
d. 3
e. 4
f. More than 4

6. Which category best indicates your years as a teacher of learners who are $\mathrm{d} / \mathrm{DHH}$ ?
a. Less than 1 year
b. 1- 2 years
c. 3-5 years
d. 6-10 years
e. 11-15 years
f. 16-20 years
g. More than 20 years

7. Which of the following best describes your highest level of education?
a. Bachelor's degree
b. Master's degree
c. Master's + additional graduate credits
d. Specialist degree
e. Doctoral degree

8. Which of the following best describes you?
a. deaf
b. Deaf
c. hard of hearing
d. hearing 
9. Which of the following best describes you?
a. American Indian or Alaska Native
b. Asian
c. Black or African American
d. Hispanic or Latino
e. Native Hawaiian or Other Pacific Islander
f. White
g. Other: Please indicate:

10. Circle the numeral that best describes your current students who are $\mathrm{d} / \mathrm{DHH}$ as appropriate to the listed categories. Circle N/A if this item is not applicable for your current teaching load or job responsibilities.

a. Students who are $\mathrm{d} / \mathrm{DHH}$ with no additional disabilities

$$
\begin{array}{llllllllll}
0 & 1 & 2 & 3 & 4 & 5 & 6 & 7 & 8 & 9
\end{array}
$$

b. Students who are $\mathrm{d} / \mathrm{DHH}$ with additional disabilities

$$
\begin{array}{llllllllll}
0 & 1 & 2 & 3 & 4 & 5 & 6 & 7 & 8 & 9
\end{array}
$$

c. N/A

11. Circle the numeral that best describes your current students who are d/DHH's primary mode of communication as appropriate to the listed categories. Circle N/A if this item is not applicable for your current teaching load or job responsibilities.
a. ASL
$\begin{array}{lllllllllll}0 & 1 & 2 & 3 & 4 & 5 & 6 & 7 & 8 & 9\end{array}$
b. Sign language with spoken language
$\begin{array}{llllllllll}0 & 1 & 2 & 3 & 4 & 5 & 6 & 7 & 8 & 9\end{array}$
c. Spoken language
$\begin{array}{llllllllll}0 & 1 & 2 & 3 & 4 & 5 & 6 & 7 & 8 & 9\end{array}$
d. N/A

12. Circle the numeral that best describes your current students who are $\mathrm{d} / \mathrm{DHH}$ as appropriate to the listed categories. Select N/A if the item is not applicable for your current teaching load or job responsibilities.
a. American Indian or Alaska Native
$\begin{array}{llllllllll}0 & 1 & 2 & 3 & 4 & 5 & 6 & 7 & 8 & 9\end{array}$
b. Asian
$\begin{array}{llllllllll}0 & 1 & 2 & 3 & 4 & 5 & 6 & 7 & 8 & 9\end{array}$
c. Black or African American
$\begin{array}{llllllllll}0 & 1 & 2 & 3 & 4 & 5 & 6 & 7 & 8 & 9\end{array}$
d. Hispanic or Latino
$\begin{array}{llllllllll}0 & 1 & 2 & 3 & 4 & 5 & 6 & 7 & 8 & 9\end{array}$
e. Native Hawaiian or Other Pacific
$\begin{array}{llllllllll}0 & 1 & 2 & 3 & 4 & 5 & 6 & 7 & 8 & 9\end{array}$ Islander
f. White
$\begin{array}{llllllllll}0 & 1 & 2 & 3 & 4 & 5 & 6 & 7 & 8 & 9\end{array}$
g. N/A
h. Other: Please indicate 


\title{
Appendix F
}

\author{
Faculty Survey Protocol
}

Faculty Survey ID:

Date Survey Sent:

Date Survey Received:

Qualitative Survey Questions:

The following questions are designed to gain an understanding of Marquardt's (2011) empowered and enabled learning phenomenon as it relates to teacher candidates' preparation to teach students who are d/DHH through this deaf education teacher preparation program. Your lived experiences and perspective are important to understanding this studied phenomenon.

1. Tell me a little about yourself, your background, and what got you interested in teaching students who are $\mathrm{d} / \mathrm{DHH}$.

2. From your perspective, at the end of student teaching, what should a teacher candidate "look like" who is ready to teach students who are d/DHH?

3. Briefly describe what a deaf education teacher candidate "looks like" who demonstrates commitment to the deaf education profession. Then answer, in what ways, if any, does this program help teacher candidates learn commitment to the Deaf education profession?

4. Briefly describe what a deaf education teacher candidate "looks like" who demonstrates proficiency in their teaching. Then answer, in what ways, if any, does this program prepare teacher candidates to be proficient in their teaching skills?

5. Briefly describe what a student who is d/Dear of hard of hearing "looks like" who is learning. Then, answer, in what ways, if any, does this program prepare teacher candidates to create learning impact in students who are $\mathrm{d} / \mathrm{DHH}$ ?

6. How could this program improve how teacher candidates are prepared to teach students who are d/DHH?

7. Please comment on the relationship between teacher candidates' hearing status and program learning. 
8. Is there anything you haven't mentioned in your responses that would be helpful for me to know?

9. Please select the appropriate answer to indicate your position at Midwest University.
a. Full-time Faculty
b. Per Course Faculty

10. Whether full-time faculty or per course faculty, which category best indicates your years teaching in this deaf education teacher preparation program?
a. Less than 1 year
b. 1-2 years
c. 3-5 years
d. 6-10 years
e. 11-15 years

11. Which of the following best describes you?
a. deaf
b. Deaf
c. hard of hearing
d. hearing

12. Which of the following best describes you?
a. American Indian or Alaska Native
b. Asian
c. Black or African American
d. Hispanic or Latino
e. Native Hawaiian or Other Pacific Islander
f. White
g. Other: Please indicate:

13. Please indicate your educational degrees, certifications, and describe your years of service within your professional area(s).

Thank you very much for agreeing to participate in this research study! As a reminder, you may withdraw from this study, including after you have completed the survey. If you decide that you want to withdraw or have questions, please contact me at KarenEngler@MissouriState.edu or my dissertation chair, Dr. Cynthia MacGregor at CMacGregor@MissouriState.edu 
Respectfully,

Karen S. Engler

Doctoral Student

University of Missouri 


\title{
Appendix G
}

\author{
Student Survey Protocol
}

\section{Student Survey ID:}

Date Survey Sent:

Date Survey Received:

Qualitative Survey Questions:

The following questions are designed to gain an understanding Marquardt's (2011) empowered and enabled learning phenomenon as it relates to your preparation to teach students who are d/DHH through this deaf education teacher preparation program. Your lived experiences and perspective are important to understanding this studied phenomenon.

1. Tell me a little about yourself, your background, and what got you interested in teaching students who are $\mathrm{d} / \mathrm{DHH}$.

2. From your perspective, at the end of student teaching, what should a teacher candidate "look like" who is ready to teach students who are d/DHH?

3. Briefly describe what a deaf education teacher candidate "looks like" who demonstrates commitment to the deaf education profession. Then answer, in what ways, if any, does/did this program help you learn commitment to the Deaf education profession?

4. Briefly describe what a deaf education teacher candidate "looks like" who demonstrates proficiency in their teaching. Then answer, in what ways, if any, does/did this program prepare you to be proficient in your teaching skills?

5. In what ways, if any, does/did this program prepare you to create learning impact in students who are d/DHH?

6. How could the program improve in preparing teacher candidates to teach students who are d/DHH?

7. Please comment on the relationship between your hearing status and the learning you experienced in this program. 
8. Is there anything you haven't mentioned in your responses that would be helpful for me to know for this research?

9. Which of the following best describes you?
a. 1 st year teacher candidate
b. 2 nd year teacher candidate
c. Deaf educator graduating in 2012
d. Deaf educator graduating in 2013

10. Which of the following best describes you?
a. deaf
b. Deaf
c. hard of hearing
d. hearing

11. Which of the following best describes you?
a. American Indian or Alaska Native
b. Asian
c. Black or African American
d. Hispanic or Latino
e. Native Hawaiian or Other Pacific Islander
f. White
g. Other: Please indicate:

12. Which of the following best describes your master's option?
a. Accelerated masters
b. Traditional masters

13. Which of the following best describes your current, assigned school which serves individuals who are $\mathrm{d} / \mathrm{DHH}$ ?
a. Public school district
b. Private school
c. School for the Deaf
d. University with university based program 
14. Which of the following indicates the number of deaf educators in your school? (If you area already a deaf educator, please count yourself in the number you select.)
a. 0
b. 1
c. 2
d. 3
e. 4
f. More than 4

15. Select the appropriate number in the pull down menu that best describes your current, assigned students who are d/DHH. Select NA if the item is not applicable for your current practicum, student teaching, or teaching load.
a. Students who are $\mathrm{d} / \mathrm{DHH}$ with no additional disabilities
b. Students who are $\mathrm{d} / \mathrm{DHH}$ with additional disabilities
c. N/A

16. Select the appropriate number in the pull down menu that best describes your current, assigned students who are d/DHH's primary mode of communication. Select NA if the item is not applicable for your current practicum, student teaching, or teaching load.
a. ASL
b. Sign language with spoken language
c. Spoken language
d. N/A

17. Select the appropriate number in the pull down menu that best describes your current, assigned students who are $\mathrm{d} / \mathrm{DHH}$. Select NA if the item is not applicable for your current teaching load or job responsibilities.
a. American Indian or Alaska Native
b. Asian
c. Black or African American
d. Hispanic or Latino
e. Native Hawaiian or Other Pacific Islander
f. White
g. Other: Please indicate:

As a reminder, if you are willing to allow me to use your Student Teaching Final Evaluation and Teaching Philosophy Statement in my research, please, if you have not already done so, send them to me as soon as possible. Again, your participation is completely voluntary. Your name will not be used and your documents will not be linked to your survey data. Data used will be added to a document protocol that will have a 
document ID, not your name on it. Also, if you choose to send you documents to me, they will be stored in a locked file cabinet separated from the folder that will have the document ID and data. If you have any questions, please contact me or my dissertation advisor, Dr. Cynthia MacGregor. My advisor may be reached at (417) 836-6046 or CMacgregor@MissouriState.edu . You may reach me, the researcher, at (417) 83666764 or KarenEngler@MissouriState.edu .

Thank you for participating in this research study by completing the survey.

Respectfully,

Karen S. Engler

Doctoral Student

University of Missouri 


\section{Appendix $\mathrm{H}$}

Program Documents Protocol

Type of Document:

Document ID:

Date Received:

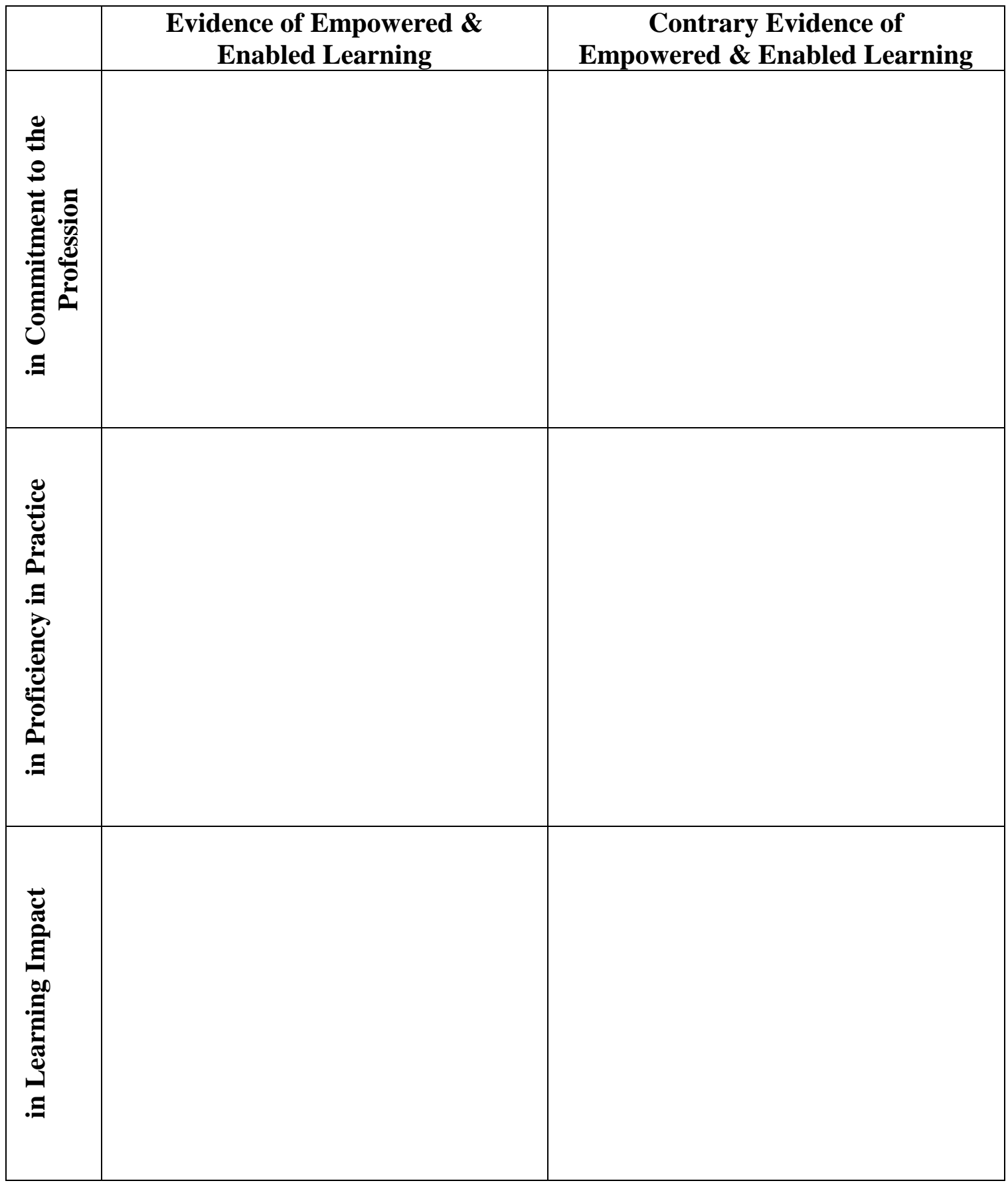

Other Comments: 


\section{Appendix I}

\section{Preschool Evaluation of Services Document Protocol}

Document ID:

Date Retrieved:

Length child had been in the preschool: ___ Less than 1 year; __ 1 year; __ More than 1 year

The following parent/family member completed quantitative and qualitative items, taken from the Preschool Evaluation of Services form, will be used to document learning impact for the preschoolers who are d/DHH.

Items receiving a rating of 3 will be used to indicate positive evidence of learning impact. Items receiving a rating of 1 will be used to indicate contrary evidence of learning impact.

Rating $1=$ Disagree $\quad$ Rating $2=$ Neutral $\quad$ Rating $3=$ Agree

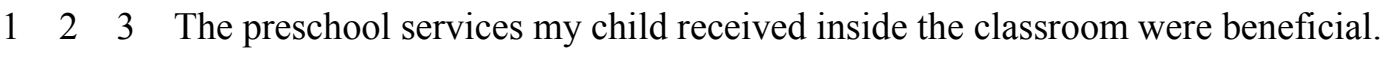

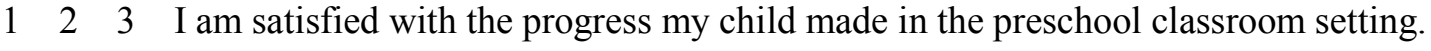

$123 \quad 3$ My child's individual needs were addressed in the classroom.

123 The teacher provided my child with a variety of experiences to promote overall development.

$\begin{array}{llll}1 & 2 & 3 & \text { The teacher is professional and courteous. }\end{array}$

$123 \quad 3$ The teacher demonstrates patience and respect when working with young children.

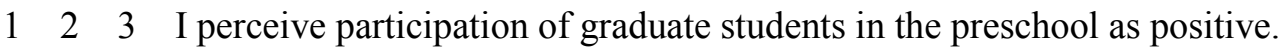

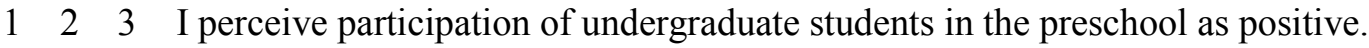

123 I felt my child's I.E.P. was adequate and appropriate regarding my child's educational needs.

$123 \quad 3$ I believe the observation room is beneficial.

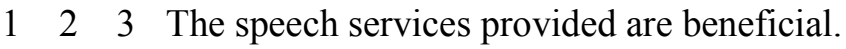

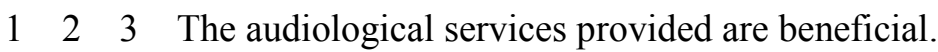

Qualitative questions regarding the most positive aspects of the preschool experience and recommended aspects of services to change will be used to measure learning impact.

\begin{tabular}{|c|c|c|}
\hline & $\begin{array}{l}\text { Evidence of Empowered \& } \\
\text { Enabled Learning }\end{array}$ & $\begin{array}{c}\text { Contrary Evidence of } \\
\text { Empowered \& Enabled Learning }\end{array}$ \\
\hline 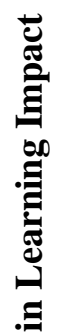 & & \\
\hline
\end{tabular}




\section{Appendix J}

\section{PRECEPTOR}

Interview Summary Sheet

ID:

Date of Summary:

\begin{tabular}{|c|c|c|c|}
\hline & $\begin{array}{c}\text { Ideal...Teacher } \\
\text { Candidate @ } \\
\text { End of Student } \\
\text { Teaching } \\
\end{array}$ & $\begin{array}{c}\text { Evidence of Empowered \& } \\
\text { Enabled Learning }\end{array}$ & $\begin{array}{c}\text { Contrary Evidence of } \\
\text { Empowered \& Enabled } \\
\text { Learning }\end{array}$ \\
\hline 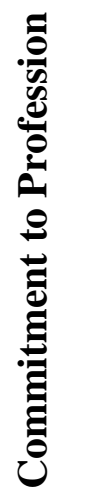 & & & \\
\hline 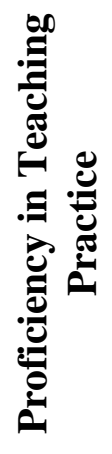 & & & \\
\hline 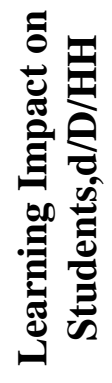 & & & \\
\hline
\end{tabular}


How the program can improve:

\section{Quotes:}

\begin{tabular}{|l|l|l|l|}
\hline Pg. & Lines & Category & \\
\hline & & & \\
& & & \\
\hline & & & \\
\hline & & & \\
\hline & & & \\
\hline & & & \\
\hline
\end{tabular}

Category Key:

$\mathrm{C}=$ Commitment to Deaf Education Profession

$\mathrm{P}=$ Proficiency in Practice

$\mathrm{L}=$ Learning Impact

$\mathrm{I}=$ Improvement 


\section{Appendix K}

FACULTY

Interview Summary Sheet

ID:

Date of Summary:

\begin{tabular}{|c|c|c|c|}
\hline & $\begin{array}{l}\text { Ideal...Teacher } \\
\text { Candidate @ } \\
\text { End of Student } \\
\text { Teaching }\end{array}$ & $\begin{array}{c}\text { Evidence of Empowered \& } \\
\text { Enabled Learning }\end{array}$ & $\begin{array}{c}\text { Contrary Evidence of } \\
\text { Empowered \& Enabled } \\
\text { Learning }\end{array}$ \\
\hline 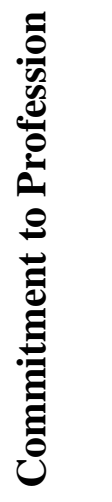 & & & \\
\hline 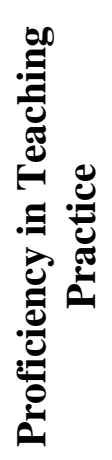 & & & \\
\hline 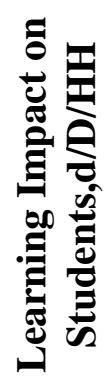 & & & \\
\hline
\end{tabular}


How the program can improve:

Quotes:

\begin{tabular}{|l|l|l|l|}
\hline Pg. & Lines & Category & \\
\hline & & & \\
& & & \\
\hline & & & \\
\hline & & & \\
\hline & & & \\
\hline & & & \\
\hline
\end{tabular}

Category Key:

$\mathrm{C}=$ Commitment to Deaf Education Profession

$\mathrm{P}=$ Proficiency in Practice

$\mathrm{L}=$ Learning Impact

$\mathrm{I}=$ Improvement 


\section{Appendix L}

\section{STUDENT}

Interview Summary Sheet

ID:

Date of Summary:

\begin{tabular}{|c|c|c|c|}
\hline & $\begin{array}{c}\text { Ideal...Teacher } \\
\text { Candidate @ } \\
\text { End of Student } \\
\text { Teaching } \\
\end{array}$ & $\begin{array}{c}\text { Evidence of Empowered \& } \\
\text { Enabled Learning }\end{array}$ & $\begin{array}{c}\text { Contrary Evidence of } \\
\text { Empowered \& Enabled } \\
\text { Learning }\end{array}$ \\
\hline 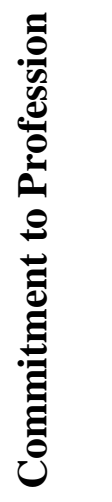 & & & \\
\hline 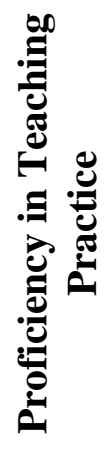 & & & \\
\hline 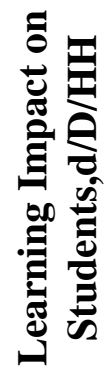 & & & \\
\hline
\end{tabular}


How the program can improve:

Quotes:

\begin{tabular}{|l|l|l|l|}
\hline Pg. & Lines & Category & \\
\hline & & & \\
& & & \\
\hline & & & \\
\hline & & & \\
\hline & & & \\
\hline & & & \\
\hline
\end{tabular}

Category Key:

$\mathrm{C}=$ Commitment to Deaf Education Profession

$\mathrm{P}=$ Proficiency in Practice

$\mathrm{L}=$ Learning Impact

$\mathrm{I}=$ Improvement 
Appendix M

\section{Student Mid-Term Evaluation/Final Evaluation}

CSD 795 - Off-site Advanced Graduate Practicum

Graduate Student:

Date:

Model Teacher/Off-site Supervisor:

MO d/Deaf and Hard of Hearing Certification (ck area):

B-12;

PK-12;

$\mathrm{K}-12$

Other Certifications:

University Supervisor CED Certification: $\_\sqrt{ }$ Parent-Infant; $\_\sqrt{ }$ Early Childhood Other Certifications: Missouri d/Deaf \& HH K-12; ECSE P-K; Speech \& Lang. Specialist K-12

Evaluation Completed By:

This evaluation tool is organized to delineate the competencies expected of graduate students in this practicum experience. The items are based on the Content Standards of the Council for Exceptional Children (CEC), the Council on the Education of the Deaf (CED), Missouri's Department of Elementary and Secondary Education Model Teacher Standards (MO-DESE, 2013), and Missouri State University's Conceptual Framework and PEC Diversity Proficiencies. All competencies align within the general frames of the professional educator: (a) commitment, (b) practice, and (c) impact (MO-DESE, 2011). Although the entry level for MO-DESE 2013 model standards is for teacher candidates at the student teaching level, the competencies included are aligned with a final practicum expectation.

Students will be evaluated at mid-term and at the end of the semester for the final. The evaluation tool will be used in a modified format for the mid-term and in its entirety for the final. For the mid-term, model teachers/off-site supervisors are asked to indicate an approximate letter grade of functioning for each of the eight categories included under the professional educator frames as well as an overall grade of functioning at the time of the mid-term. Comments supporting the need for improvement, as well as strengths, are encouraged to be included under the comments and/or summary sections within each category.

For the final evaluation, provide a number score for each item within the categories. Quality of competency demonstration should coincide with expectations at a final graduate level practicum experience prior to student teaching and be a consideration in grading. The scores entered will be totaled automatically. The number scores correlate to letter grades as follows: 
$A=4$ Practicum student consistently demonstrates the competency $90 \%$ or more of the time.

$\mathrm{B}=3$ Practicum student demonstrates the competency $80-89 \%$ of the time. $\mathrm{C}=2$ Practicum student demonstrates the competency $70-79 \%$ of the time.

N/A Not applicable for current practicum setting. 
1. Maintains an appropriate appearance within the context of the practicum setting: neat, well-groomed, appropriate dress

2. Maintains tact, patience, consideration, emotional control, temperament, freedom from mannerisms, alertness, sense of humor.

3. Demonstrates initiative and independence (e.g., contacts model teacher for information or exploration of ideas, suggests ideas for instructional activities, reviews model teacher's materials and resources for developing instructional activities).

4. Demonstrates confidence/poise

5. Attends practicum reliably

6. Is punctual and dependable

7. Upholds high standards of competence, ethics, and integrity and exercises sound judgment in the practice of the profession.

8. Conducts professional activities in compliance with roles, responsibilities and applicable laws and policies.

9. Demonstrates commitment to developing the highest educational and quality-of-life potential of individuals with exceptional learning needs.

10. Demonstrates sensitivity for the culture, language, religion, gender, disability, socio-economic status, and sexual orientation of individuals. PEC Diversity Proficiency: 5 Respect

\begin{tabular}{|l|l|}
\hline & \\
\hline & \\
\hline & \\
\hline & \\
\hline & \\
\hline & \\
\hline & \\
\hline & \\
\hline
\end{tabular}

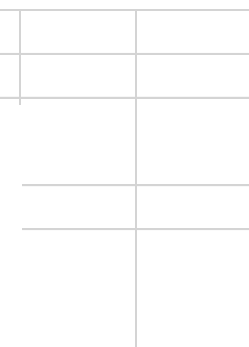

|

11. Uses written language effectively (e.g., lesson plans, emails, etc.).

12. Receptive and responsive to supervision

13. Seminar participation

14. Binder organization \& timeliness

Personal Attributes \& Professionalism Grade

$0 / 52$ 


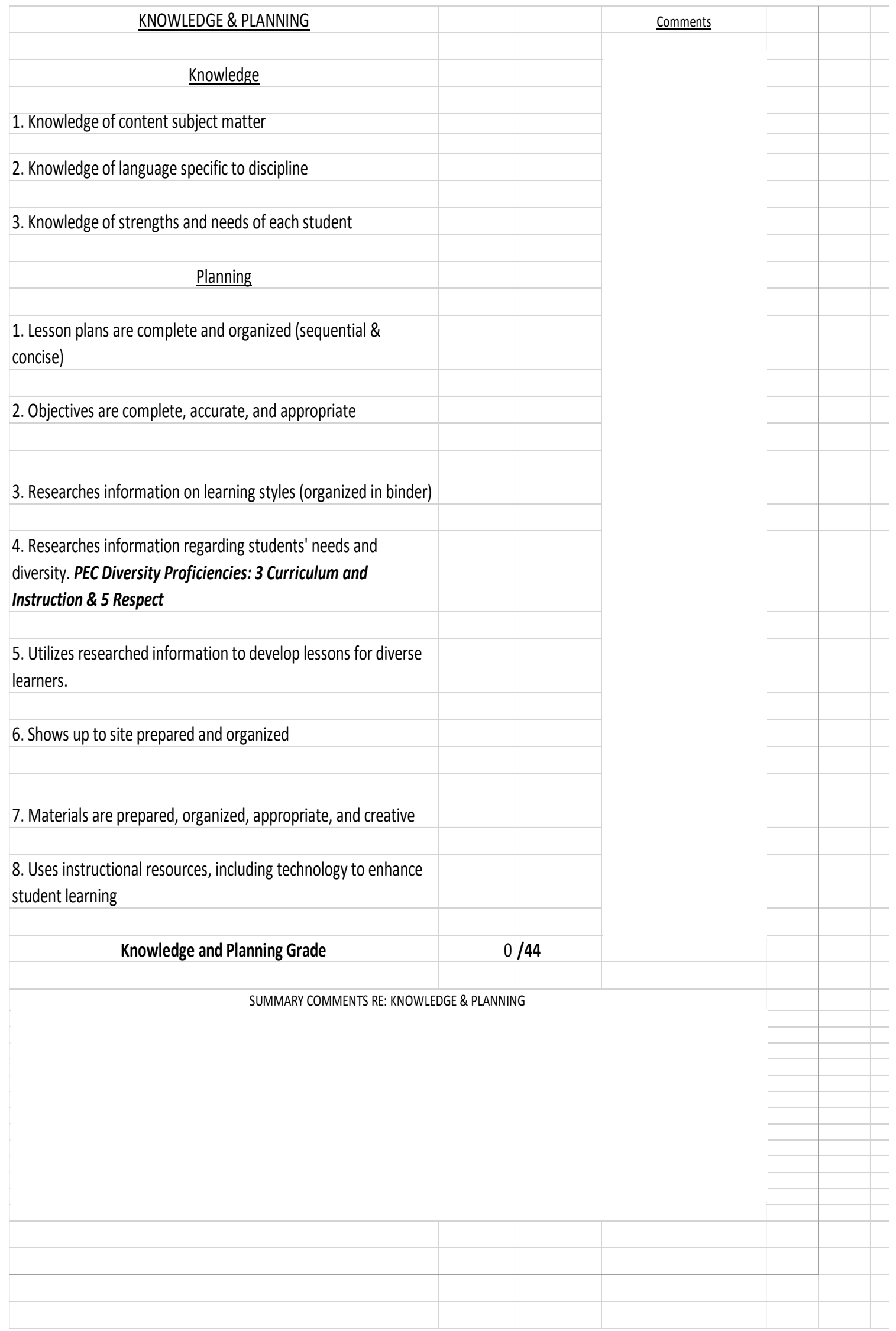




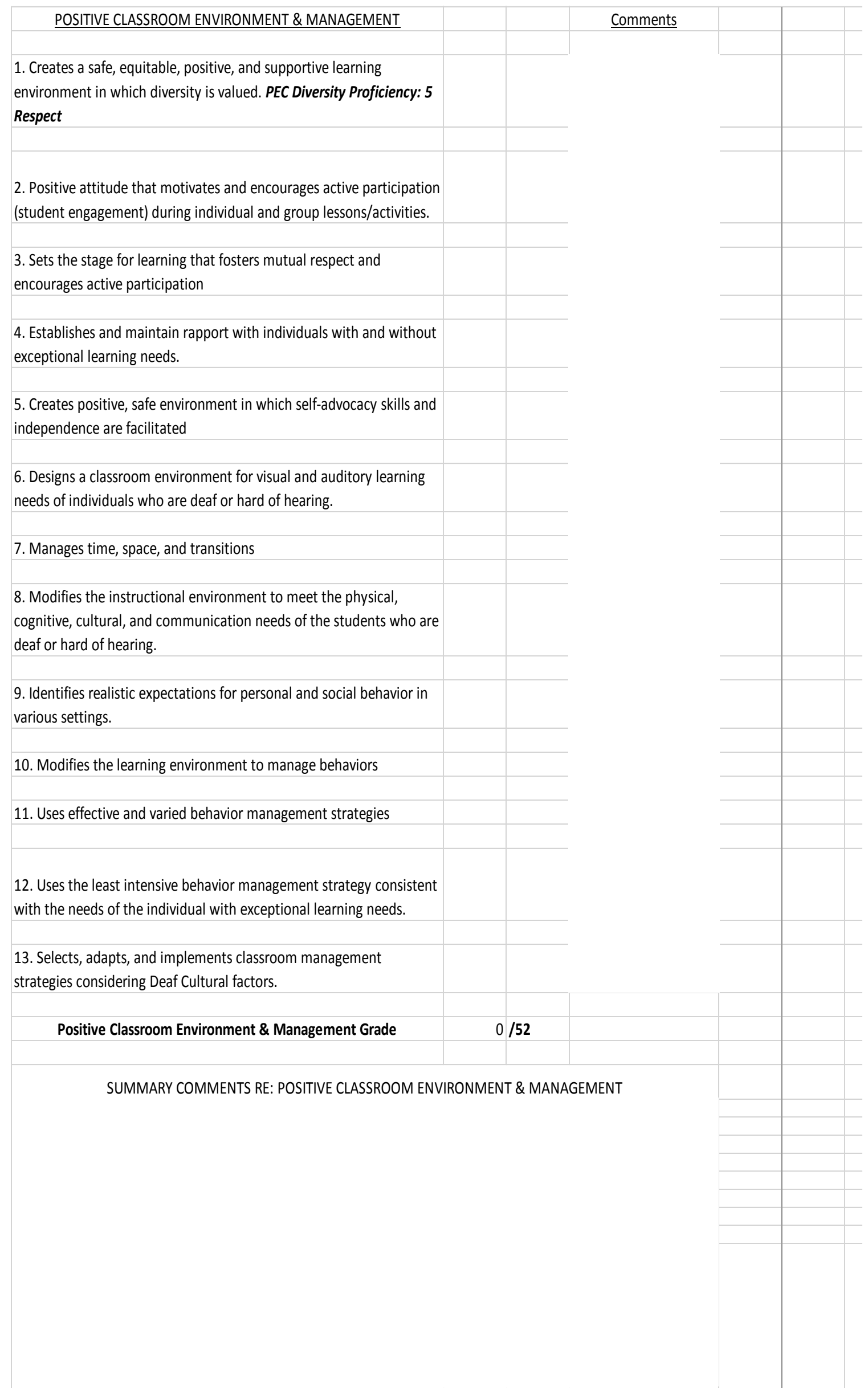




\begin{tabular}{|l|l|l|}
\hline \multicolumn{1}{|c|}{ Effective Communication } \\
\hline $\begin{array}{l}\text { 1. Uses strategies to support and enhance communication } \\
\text { skills of individuals with exceptional learning needs. PEC } \\
\text { Diversity Proficiency: } 3 \text { Curriculum and Instruction }\end{array}$ \\
\hline $\begin{array}{l}\text { 2. Uses communication strategies and resources to facilitate } \\
\text { understanding of subject matter for students whose primary } \\
\text { language is not the dominant language. PEC Diversity } \\
\text { Proficiency: } 2 \text { Communication }\end{array}$ \\
\hline $\begin{array}{l}\text { 3. Gathers and analyzes verbal and nonverbal communication } \\
\text { samples. }\end{array}$ \\
\hline 4. Facilitate independent communication \\
\hline 5. Effectively communicates with school personnel \\
\hline
\end{tabular}

7. Proficiency in sign language. PEC Diversity Proficiency: 2

Communication

\section{Comments}

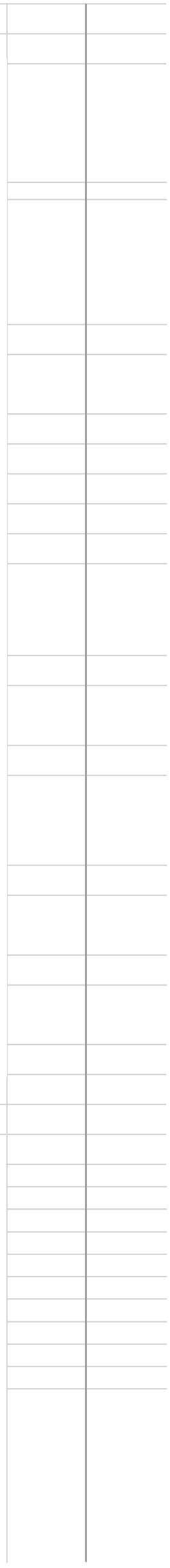

8. Creates opportunities for auditory and visual learning as appropriate to individual students. PEC Diversity Proficiency: 2 Communication

9. Manages assistive technology (listening device check \& Ling Sound check)

10. Initiates obtaining supervisory assistance as appropriate.

Effective Communication Grade

$0 / 40$ 


\begin{tabular}{|c|c|c|}
\hline Implementation & & Comments \\
\hline $\begin{array}{l}\text { 1. Implements instruction for diverse learners, including } \\
\text { learners with multiple disabilities (if applicable). PEC } \\
\text { Diversity Proficiencies: } 3 \text { Curriculum and Instruction \& } \\
4 \text { Resources }\end{array}$ & & \\
\hline 2. Implements learning objectives & & \\
\hline 3. Presents subject matter in a way that is meaningful & & \\
\hline $\begin{array}{l}\text { 4. Presents subject matter in a way that is engaging and } \\
\text { maintains on task behavior }\end{array}$ & & \\
\hline $\begin{array}{l}\text { 5. Strong, complete introduction to } \\
\text { lesson/motivates/sparks interest, making learning } \\
\text { connection, letting them in on learning-What? Why? }\end{array}$ & & \\
\hline $\begin{array}{l}\text { 6. Lessons demonstrate diversity is respected and } \\
\text { valued. PEC Proficiency: } 5 \text { Respect }\end{array}$ & & \\
\hline $\begin{array}{l}\text { 7. Uses visuals to support learning of students who are } \\
\text { d/Deaf or hard of hearing }\end{array}$ & & \\
\hline $\begin{array}{l}\text { 8. Promotes the development of speech and auditory } \\
\text { skills (as appropriate) }\end{array}$ & & \\
\hline $\begin{array}{l}\text { 9. Integrates affective, social and life skills within } \\
\text { academic curricula }\end{array}$ & & \\
\hline 10. Incorporates technology within lessons & & \\
\hline 11. Uses instructional time effectively & & \\
\hline $\begin{array}{l}\text { 12. Makes responsive adjustments to instruction based } \\
\text { on continual observations. }\end{array}$ & & \\
\hline $\begin{array}{l}\text { 13. Selects, adapts, and uses instructional strategies } \\
\text { and materials according to characteristics of the } \\
\text { individual with exceptional learning needs. } \\
\text { 14. Uses strategies to facilitate maintenance and } \\
\text { generalization of skills across learning environments. }\end{array}$ & 271 & \\
\hline
\end{tabular}


10. Incorporates technology within lessons

11. Uses instructional time effectively

12. Makes responsive adjustments to instruction based on continual observations.

13. Selects, adapts, and uses instructional strategies and materials according to characteristics of the individual with exceptional learning needs.

14. Uses strategies to facilitate maintenance and generalization of skills across learning environments.

15. Uses procedures to increase the individual's selfawareness, self-management, self-control, self-reliance, and self-esteem.

16. Uses strategies that promotes successful transitions for individuals with exceptional learning needs. (From lesson to lesson, or to various education settings.

\section{Applies first and second language teaching} strategies to the needs of the individual (as applies to current setting).

18. Includes closing to lesson (Make it count; sum it up; "high 5"; entice for more)

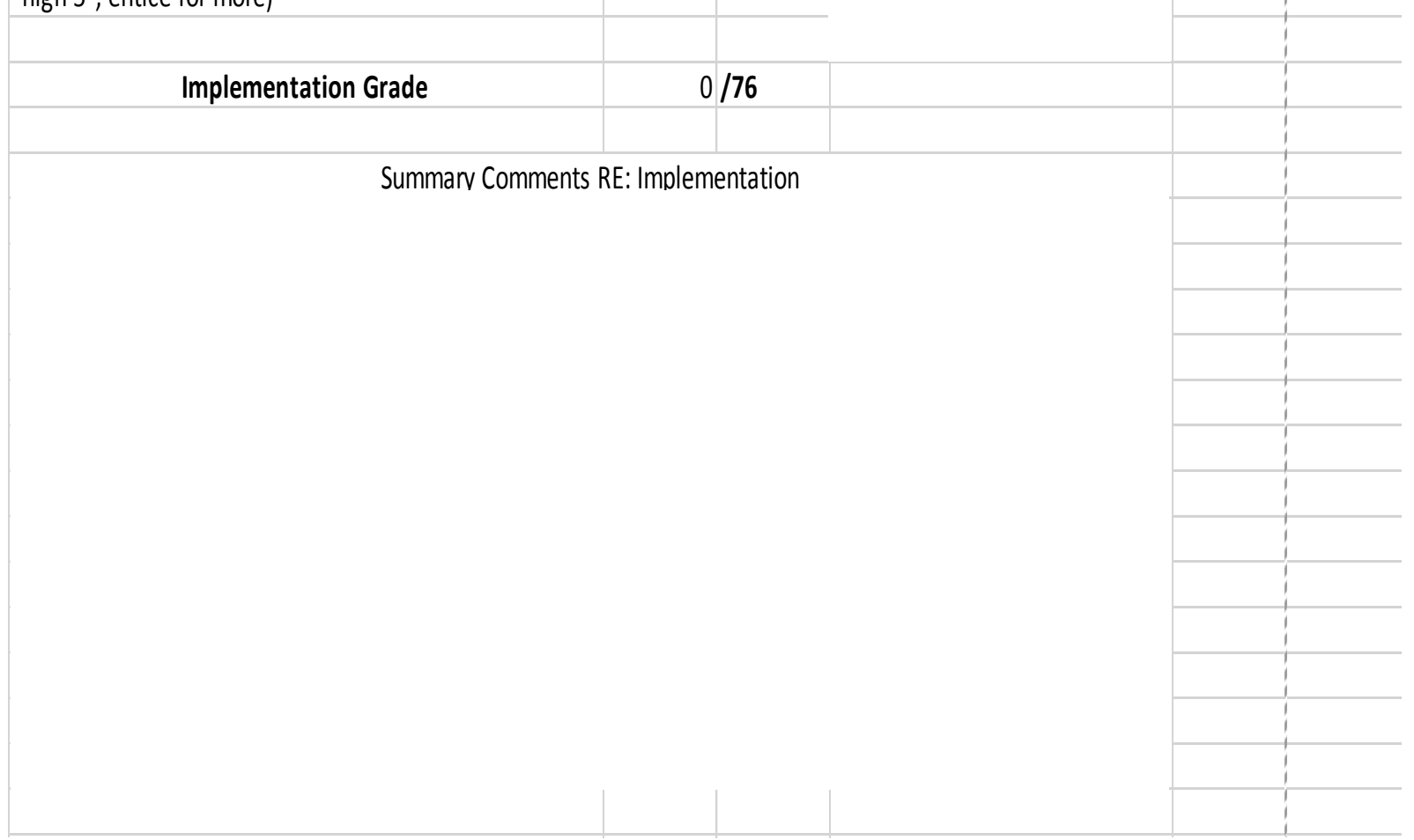




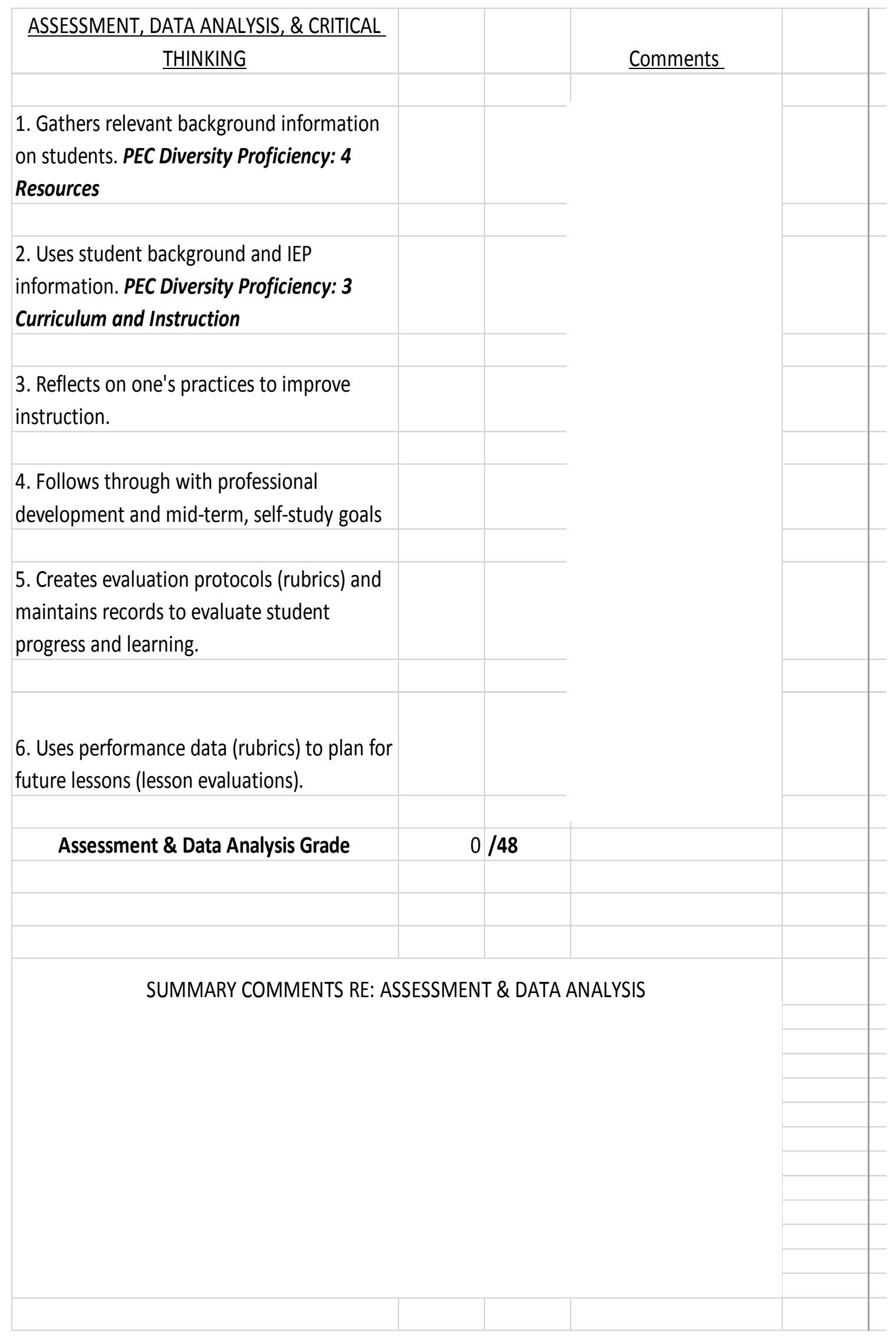




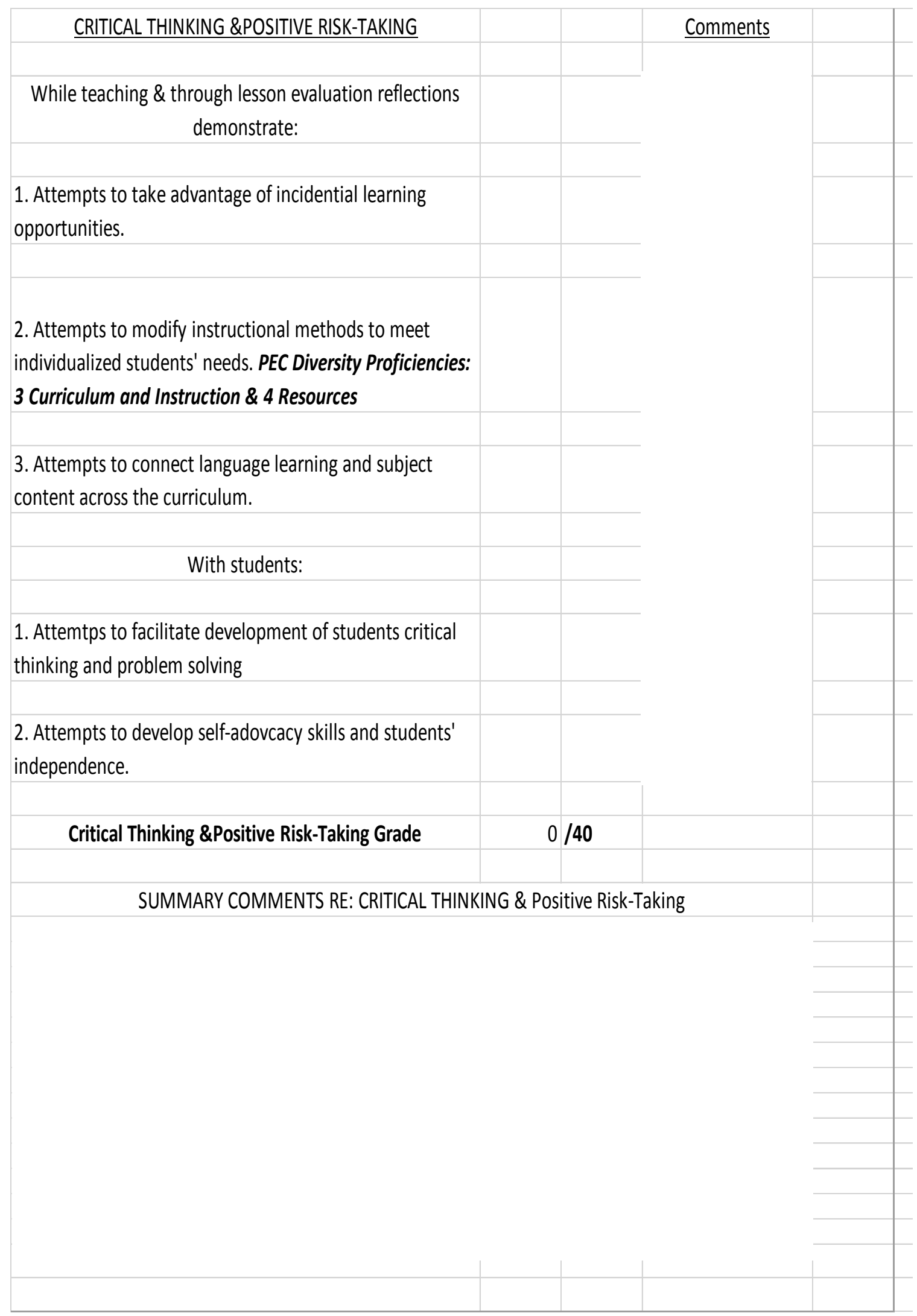


1. Through cooperative partnership with on-site model teacher/supervisor, address a real-life challenge within the school community. (see 9th week activity)

2. Collaborate with school professionals (+ other resources) to gather information for preparing students to use interpreters.

3. Reflective journals on 3-hr. observations at 2 different schools with students who are d/Deaf or hard of hearing re: teaching strategies, classroom management strategies, and teaching students to use intrepreters and use of interpreters.

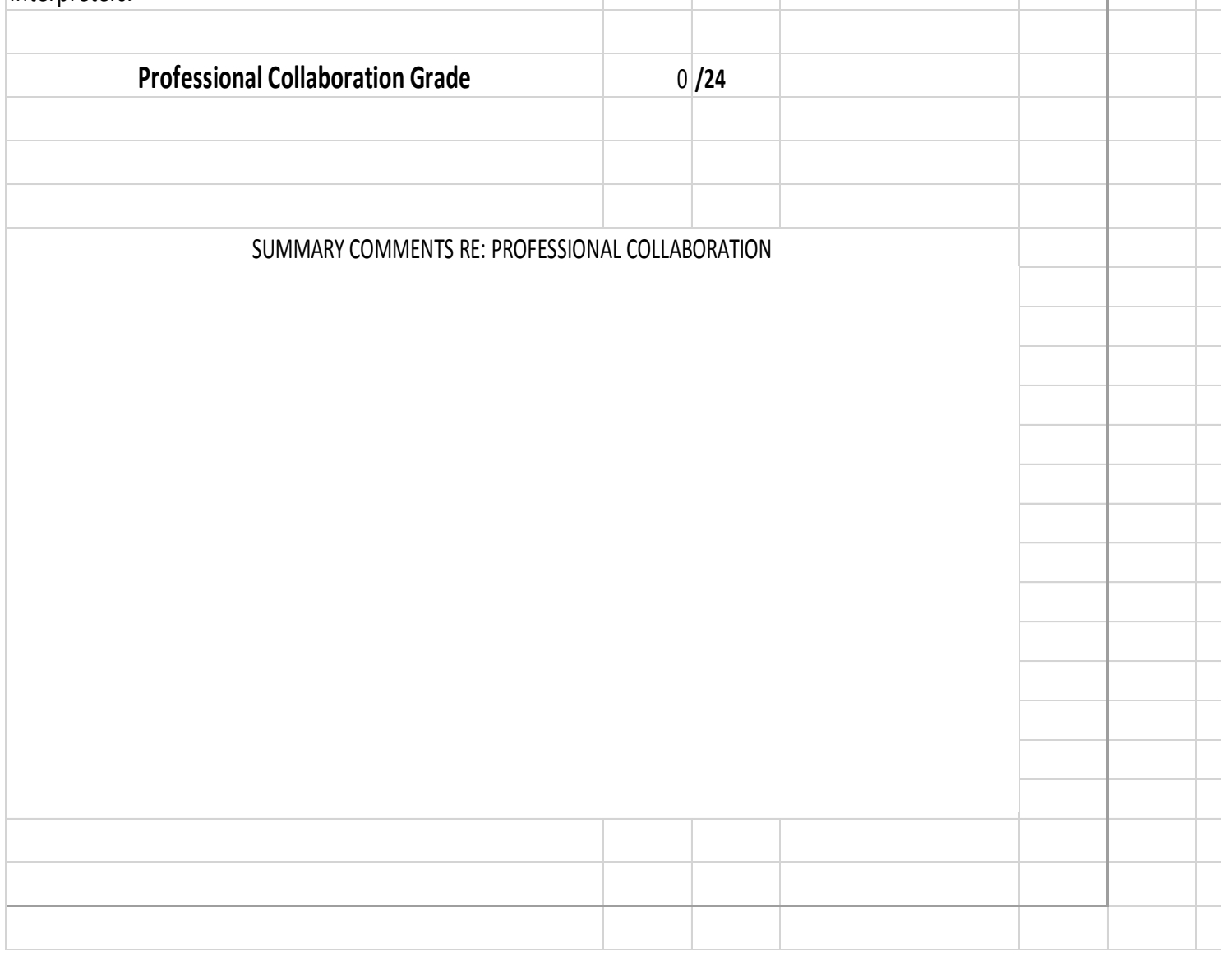




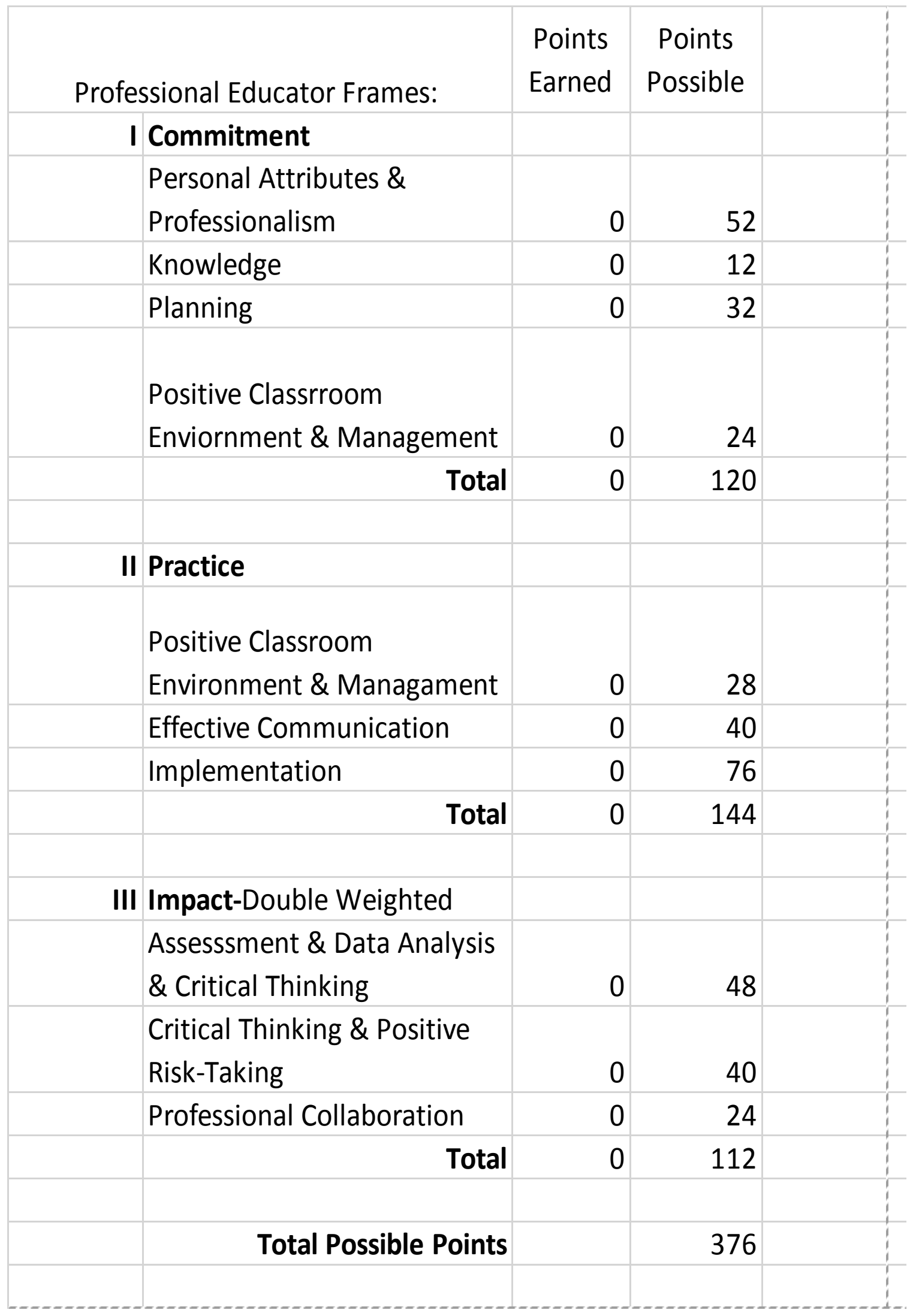




\begin{tabular}{|l|l|l|}
\hline Grade & \multicolumn{1}{|c|}{$\%$} & \multicolumn{1}{|c|}{ Criteria } \\
\hline A+ & $98-100$ & Rare performance. Reserved for highly exceptional, rare achievement. \\
\hline$A$ & $94-97$ & Excellent. Outstanding achievement. \\
\hline A- & $91-93$ & Excellent work, but not quite outstanding. \\
\hline B+ & $88-90$ & Very good. Solid achievement expected of most graduate students. \\
\hline$B$ & $81-87$ & Good. Acceptable achievement. \\
\hline & & \\
\hline C+ & $78-80$ & Fair achievement, above minimally acceptable level. \\
\hline C & $74-77$ & Fair achievement, but only minimally acceptable. \\
\hline C- & $71-73$ & Low performance. \\
\hline D & $61-70$ & $\begin{array}{l}\text { Very low performance. These records of students receiving such grades are } \\
\text { subject to review. }\end{array}$ \\
\hline F & $\begin{array}{l}60 \& \\
\text { below }\end{array}$ & Failure. \\
\hline
\end{tabular}

Practicum Grade/Letter Grade@ Mid-Term

Practicum Grade/Letter Grade@ Final

Total Practicum Grade: (average of mid-term \& final)

Signature of Model Teacher or University

Signature of Graduate Student

Date

Supervisor 
Karen Engler was born in Florissant, Missouri. She grew up in Springfield, Missouri with her parents and three sisters. Her parents, Richard and Jackie Hagenhoff, valued faith, family, and education for which Karen will be forever grateful. Karen graduated from St. Agnes Grade School in 1973 and Springfield Catholic H.S. in 1977. She and her sisters were first generation college graduates.

Karen earned three degrees from Missouri State University: (a) A.S. in Child Development, (b) B.S. in Communication Disorders, and (c) M.A. in Communication Sciences \& Disorders, Education of the Deaf. Karen completed her EdD in Educational Leadership and Policy Analysis from the University of Missouri in 2014.

For over 20 years, Karen has served children who were d/Deaf or hard of hearing and their families and continues to teach undergraduate and graduate students as a clinical professor at a Midwest university. Karen also serves as a cochlear implant educational consultant to public school district personnel throughout the state. In addition, Karen co-authored a book chapter on early intervention as well as presented internationally on early intervention and a cochlear implant consultation model that she developed to support students with cochlear implants within their rural school districts.

Karen and her wonderful husband, John, have been married for 32 years. They have four amazing children. Faith, family and education are pivotal in their lives. Like Karen and John, all four children graduated from Springfield Catholic; they are currently enrolled in universities. Zach is attending graduate school at Missouri State University. Jenna is attending graduate school at the University of San Diego. Nicholas and Michael are working toward undergraduate degrees at Rockhurst University in Kansas City, MO. Karen's mother-in-law, Sis Engler (1926-2008), was also instrumental in her life. 